\title{
Experimental Wind Tunnel Testing and Numerical Optimization Studies for Airfoil Trailing Edge Noise Reduction
}

\author{
by \\ Basim Al Tlua \\ A thesis submitted to \\ the Faculty of Graduate and Postdoctoral Affairs \\ in partial fulfillment of the requirements for the degree of
}

Doctor of Philosophy

in

\author{
Mechanical and Aerospace Engineering \\ Ottawa-Carleton Institute for Mechanical and Aerospace Engineering \\ Department of Mechanical and Aerospace Engineering \\ Carleton University \\ Ottawa, Ontario, Canada, K1S 5B6
}

August 2021

(C)2021, Basim Al Tlua 
The undersigned recommend to

the Faculty of Graduate and Postdoctoral Affairs

acceptance of the thesis

Experimental Wind Tunnel Testing and Numerical Optimization Studies for Airfoil Trailing Edge Noise Reduction

submitted by

Basim AL Tlua, M.Sc

in partial fulfillment of the requirements for

the degree of Doctor of Philosophy in Mechanical and Aerospace Engineering

Chair, Ron Miller, Department of Mechanical and Aerospace Engineering

Thesis Supervisor, Professor Joana Rocha

Committee Members, Professor Fidel Khouli and Tarik Kaya

Member of Joint Institute, Professor James Gerald McDonald, Mechanical Engineering-University of Ottawa

External Examiner, Professor Sebastian Ghinet, Research Officer-National Research Council of Canada

Carleton University

August, 2021 


\begin{abstract}
Aircraft noise is an important topic of research, as researchers have been seeking ways to reduce it. The current thesis aims to advance the state of the art of airfoil trailingedge (TE) noise reduction, by experimentally testing numerically optimized TE designs studied by the author, and obtained from semi-empirical models prediction. Different configurations of a non-flat plate, NACA-0012 airfoil, and flat-plate trailing-edge serrations are investigated. A wind tunnel test section that provides the required environment is designed and created. The test section walls have been acoustically treated to simulate an acoustically far-field environment with forwarding flight. The two sides of the wind tunnel test section are fitted with anechoic chambers and lined with acoustic transparency tensioned cloth screens which act as an interface between the test section and the anechoic chambers to provide a smooth flow surface while eliminating the need for a jet catcher and reducing interference effects. Its aeroacoustic performance is measured. Results show that background noise is comparable with other aeroacoustic wind tunnels worldwide. A straight TE airfoil and flat plate were tested as a benchmark, and then compared with serrated trailing-edge geometries. Results show that the serration geometry is effective in reducing noise and that noise radiated from the TE is at least $6 \mathrm{~dB}$ higher than the background noise, satisfying the requirements for aeroacoustic measurements.

The ability of the trailing-edge serrations to reduce TE noise is examined through numerical optimization study. Three different serration geometries are optimized for the overall noise from $0.1 \mathrm{kHz}$ to $10 \mathrm{kHz}$. The noise spectra was initially modeled using semi-empirical models by Howe, for a semi-infinite flat plate, at zero angle of attack and at low Mach numbers. The single-size sawtooth optimization study found the optimum performing TE sawtooth geometry (in terms of noise reduction), which was confirmed experimentally. The measurements confirmed that numerical optimization predicted a much larger noise reduction compared to measured values. Comparison of single-size sawtooth, slit and sinusoidal TE designs show that, while the three geometries reduced
\end{abstract}


noise from a straight TE, sawtooth serrations result in larger noise reduction than singlesize slit and sinusoidal serrations. Further, it was found that the single-size sawtooth TE that produces the overall least noise has the largest feasible amplitude and smallest feasible width.

Noise radiation from optimized trailing-edges with four different sawtooth serrations have been investigated. The noise spectra was modeled using semi-empirical models by Lyu, for a semi-infinite flat plate, at zero angle of attack and at low Mach numbers. It has been shown that the sawtooth TE serration design can have a significant effect on the level of noise reduction. The optimum single-size of sawtooth tends to maximum amplitude $h_{o p t}=h_{\max }$, and minimum wavelength, $\lambda_{o p t}=\lambda_{\min }$. Moreover, the greatest noise reduction of up to $12.89 \mathrm{~dB}$ over a wider range of frequencies was found with one of the configurations. Numerical and experimental results are in agreement that optimized serrated trailing-edge configurations can yield less TE noise compared to the traditional straight trailing-edge configuration. The optimization studies underestimated the measurements by about 2 to $12 \mathrm{~dB}$, but the overall SPL over the frequency spectrum for the different serration geometries is very suggestive of good agreement comparing with the previous study [28]. The results are promising and further investigations to upgrade the models could potentially reduce or eliminate the underestimation. 


\section{Acknowledgments}

I would like to greatly thank all people who either contributed to the work described with this thesis or to me personally during the working time on it. First and foremost, I would like to express my gratitude and appreciation to my supervisor professor Joana Rocha. Her continuous support and guidance have significantly contributed to my research progress during my $\mathrm{PhD}$.

Without a scholarship from my home country Libya, it would have been difficult or impossible to achieve this work. I wish all progress and prosperity to my country and peace to all people.

Many thanks to all friends for their emotional supports and invaluable advises. Their encouragement during more difficult phases of my study has inspired me and made me more productive.

Finally, I dedicate this thesis to my family; my beloved mother and father whose love

continues as long as my heart is beating, my lovely wife, Maleka, for always standing by me and our kids, my friend Ada Grieco with respect. 


\section{Preface}

As dened by section 12.4 of 2021-2022 Graduate Calendar, the current thesis is an integrated Ph.D thesis. It includes the following articles which are either published or are submitted:

1. B. Al Tlua, and J. Rocha, Development and Testing of an Aeroacoustic Wind Tunnel Test Section, Acoustics Week in Canada 2019, Edmonton, AB, Canada, Oct. 8-11, 2019.

2. B. Al Tlua, and J. Rocha (2020), Optimization and Testing of Flat-Plate Trailing Edge Serration Geometry for Reducing Broadband Airfoil Self-Noise, Canadian Acoustics, 48(4):7-17.

3. B. Al Tlua, and J. Rocha, Numerical Optimization and Wind Tunnel Testing of Trailing Edge Serrations for Reducing Airfoil Self-Noise, 27th International Congress on Sound and Vibration (ICSV27), Prague, Czech Republic, Jul. 11-16, 2021.

4. B. Al Tlua, and J. Rocha, Aeroacoustic Design Optimization and Experimental Investigation of Sawtooth Trailing Edges for Maximized Noise Reduction, Applied Acoustics Journal, (Submitted, and Under Review).

5. Y. Salama, B. Al Tlua, and J. Rocha, ELES and Tonal Noise Prediction of SerratedTE Airfoils, MDPI Journal, Aerospace Journal (Submitted, and Under Review).

6. B. Al Tlua, and J. Rocha, Experimental Study of NACA-0012 Airfoil Instability Noise with Sawtooth Trailing Edges, Wind Engineering Journal, (Submitted, and Under Review). 
The format of these journal papers has been transformed to be more coherent to the format of the current thesis. Also, the images and plots have been re-centred and rescaled. Section of additional work have also been added in the Appendix. The references have been created in a single list that can be found at the end of this thesis. 


\section{Contents}

\begin{tabular}{lll}
\hline Abstract & i
\end{tabular}

\begin{tabular}{|ll}
\hline Acknowledgments & iii
\end{tabular}

List of Figures $\quad$ xi

$\begin{array}{ll}\text { List of Tables } & \text { xvi }\end{array}$

List of Acronyms $\quad$ xvii

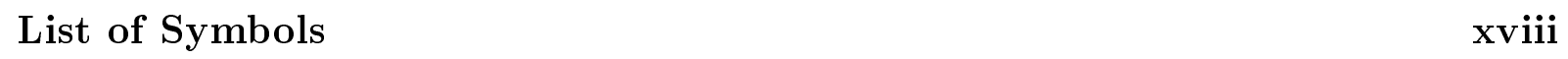

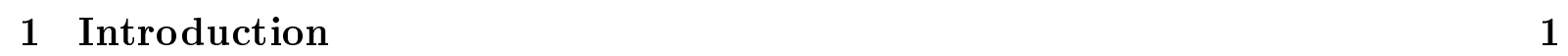

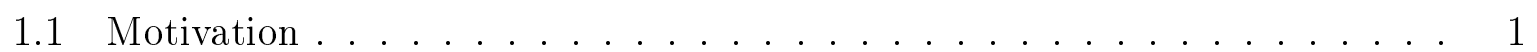

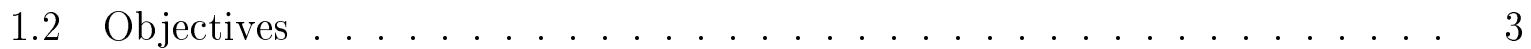

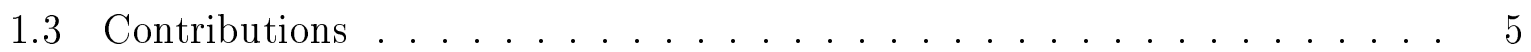

1.4 Outline . . . . . . . . . . . . . . . . . . . . 8

2 Background and Literature Review 19

2.1 Airfoil Self-Noise Mechanisms . . . . . . . . . . . . . . . . . . 9

2.2 Airfoil Trailing-Edge Noise Reduction . . . . . . . . . . . . . . . . . 11

$2.2 .1 \quad$ Biomimetics on Serration Design . . . . . . . . . . . . 12 


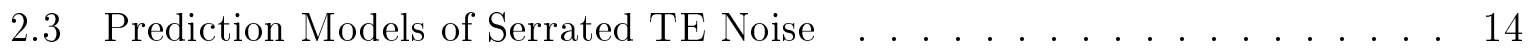

2.4 Design Optimization for TE Noise Reduction $\ldots \ldots \ldots \ldots$

$2.5 \quad$ Previous Experimental Investigations of the TE Noise $\ldots \ldots \ldots \ldots$

3 Optimization and Testing of Flat-Plate Trailing-Edge Serration Geom$\begin{array}{ll}\text { etry for Reducing Airfoil Self-Noise } & 19\end{array}$

3.1 Introduction . . . . . . . . . . . . . . . . . . . . 20

3.2 Wind tunnel characterization . . . . . . . . . . . . . . . 22

3.3 New aeroacoustic test section $\ldots \ldots \ldots \ldots \ldots$

$3.4 \quad$ Anechoic system . . . . . . . . . . . . . . . . . . . 24

$3.4 .1 \quad$ Design considerations . . . . . . . . . . . . . . . . . . . 24

3.4 .2 Physical layout $\ldots \ldots \ldots \ldots \ldots$

$3.4 .3 \quad$ Acoustic transparency of the cloth windows $\ldots \ldots \ldots \ldots$

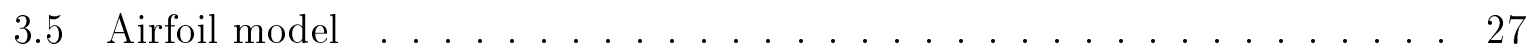

3.6 Noise source model . . . . . . . . . . . . . . . . . . . . . . . . . 28

3.7 Numerical optimization methods $\ldots \ldots \ldots \ldots$

3.8 Results and discussion $\ldots \ldots \ldots \ldots \ldots$

$3.8 .1 \quad$ Background noise levels $\ldots \ldots \ldots \ldots$. . . . . . . . . 34

3.8 .2 Far-field trailing edge noise measurements $\ldots \ldots \ldots \ldots$

3.8.2.1 Measurements of the airfoil TE noise . . . . . . . . . 37

3.8 .2 .2 Measurements of the flat-plate TE noise $\ldots \ldots$. . . 38

$3.8 .3 \quad$ Optimization results $\ldots \ldots \ldots \ldots \ldots$

3.8.3.1 Verification of the noise model . . . . . . . . . . . . 41

3.8.3.2 Optimization of single-size sawtooth serrations _ . . . . 43

3.8.3.3 Optimization of single-size slitted serrations . . . . . . . 44

3.8.3.4 Optimization of single-size sinusoidal serrations . . . . . 45

3.8.4 Experimental results and comparison with numerical results . . . 47

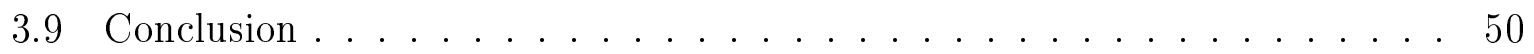


4 Aeroacoustic Design Optimization and Experimental Investigation of Sawtooth Trailing Edges for Maximized Noise Reduction 52

4.1 Introduction . . . . . . . . . . . . . . . . . . . . . . 53

4.2 Sawtooth TE Sound Model . . . . . . . . . . . . . . . . . . . . . . . 54

4.3 Numerical Optimization Method . . . . . . . . . . . . . . . . . . 60

$4.3 .1 \quad$ Definition of the optimization problem . . . . . . . . . . . 60

4.4 Optimization Results $\ldots \ldots \ldots \ldots$

4.4 .1 Noise model verification . . . . . . . . . . . . . . . . . 62

$4.4 .2 \quad$ Optimization of sawtooth serrations . . . . . . . . . . . 63

$4.5 \quad$ Experimental Setup . . . . . . . . . . . . . . . . . . . 67

4.5 .1 The wind tunnel $\ldots \ldots \ldots \ldots \ldots$. . . . . . . . . . . 67

4.5 .2 Flat-plate model with TE serrations $\ldots \ldots \ldots$. . . . . . 68

4.5 .3 Airfoil model with TE serrations . . . . . . . . . . . . . . 69

4.5 .4 Instruments and procedure . . . . . . . . . . . . 70

4.5.4.1 Data Acquisition System . . . . . . . . . . . . 70

4.5 .4 .2 Microphone Measurement System . . . . . . . . . . . 71

4.5 .4 .3 Microphone calibration . . . . . . . . . . . . . . 72

4.5.4.4 Data analysis procedure . . . . . . . . . . . 73

4.6 Comparison of experimental data with optimization results . . . . . . . . 74

4.7 Conclusion $\ldots \ldots \ldots \ldots \ldots \ldots \ldots$

5 ELES and Tonal Noise Prediction of Serrated-TE Airfoils 80

5.1 Introduction . . . . . . . . . . . . . . . . . . . . 81

5.2 Numerical Methodology . . . . . . . . . . . . . . . . . . . . 87

5.2 .1 Governing Equations $\ldots \ldots \ldots \ldots$. . . . . . . . . 87

5.3 RANS/LES Interface $\ldots \ldots \ldots \ldots$

5.4 The FW-H Acoustic Analogy $\ldots \ldots \ldots$

$5.5 \quad$ Flow Configuration and Computational Setup . . . . . . . . . . . . 93 
$5.5 .1 \quad$ Flow Configuration . . . . . . . . . . . . . . . . . 93

$5.5 .2 \quad$ Computational Mesh and Setup . . . . . . . . . . . . . 96

$5.5 .3 \quad$ Experimental Setup . . . . . . . . . . . . . . . . 100

5.6 Results . . . . . . . . . . . . . . . . . . . . . . . . . . . . . 102

5.6 .1 Surface Pressure $\ldots \ldots \ldots \ldots$. . . . . . . . . . . . 102

5.6 .2 Wake Characteristics . . . . . . . . . . . . . . . . . . . 105

5.6 .3 Far-field Noise . . . . . . . . . . . . . . . . . . . . . . . 111

5.7 Conclusion . . . . . . . . . . . . . . . . . . 116

6 Experimental Investigation of NACA-0012 Airfoil Instability Noise with

$\begin{array}{ll}\text { Sawtooth Trailing Edges } & 119\end{array}$

6.1 Introduction . . . . . . . . . . . . . . . . . . . . . . . 120

6.2 Experimental setup . . . . . . . . . . . . . . . . . . . . . . . . 122

6.2 .1 Airfoil model and TE serrations . . . . . . . . . . . . . . 122

6.2 .2 Wind tunnel facility . . . . . . . . . . . . . . . . . 124

6.2 .3 Instruments and procedures $\ldots \ldots \ldots \ldots$. . . . . . . . 124

6.3 Results . . . . . . . . . . . . . . . . . . . . . . . 126

6.3 .1 Occurrence of tones . . . . . . . . . . . . . . . . 126

$6.3 .2 \quad$ Airfoil instability noise with a straight TE $\ldots \ldots \ldots$. . . . . . 128

6.3 .3 Effect of the boundary layer tripping $\ldots \ldots \ldots \ldots$

$6.3 .4 \quad$ Noise reduction by sawtooth TEs . . . . . . . . . . . . . 131

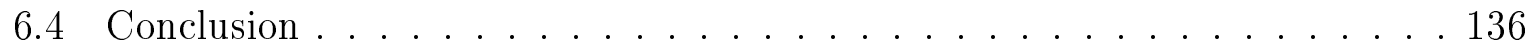

7 Conclusions and Recommendations for Future Work 137

7.1 Conclusions . . . . . . . . . . . . . . . . . . . . . . . . 139

7.1.1 Optimization and Testing of Flat-Plate Trailing-Edge Serration Geometry for Reducing Airfoil Self-Noise . . . . . . . . . . . . 139 
7.1.2 Aeroacoustic Design Optimization and Experimental Investigation of Sawtooth Trailing-Edges for Maximized Noise Reduction . . . . . 140

7.1.3 ELES and Tonal Noise Prediction of Serrated TE Airfoils . . . . . . 141

7.1.4 Experimental Investigation of NACA-0012 Airfoil Instability Noise with Sawtooth Trailing-Edges . . . . . . . . . . . . . . . . . 142

7.2 Future Work . . . . . . . . . . . . . . . . . . . . . 143

$7.2 .1 \quad$ Numerical Analysis and Optimization . . . . . . . . . . . . . 143

7.2 .2 Experimental Studies . . . . . . . . . . . . . . . . 144

\begin{tabular}{ll}
\hline References & 146
\end{tabular}

\begin{tabular}{|ll}
\hline A Measurement Uncertainty & 163
\end{tabular} 


\section{List of Figures}

2.1 Airfoil self-noise mechanisms as defined by Brooks et al. $|1|$. . . . . . . . . 11

2.2 Structure of the Owl's wing in different magnifications showing the structure of the TE fringe (from Bachmann et al. |2|). . . . . . . . . . . . . 14

$3.1 \quad$ Wind tunnel at Carleton University. . . . . . . . . . . . . . . . . . . . 23

3.2 Aeroacoustic test section. . . . . . . . . . . . . . . . . 24

3.3 Right: a cross-section through the aeroacoustics test section and anechoic as seen from above. Left: photograph was taken from downstream showing the test section interior. . . . . . . . . . . . . . . . . . 24

3.4 Acoustic transparency of the cloth window. . . . . . . . . . . . 26

3.5 Attenuation of sound passing through the acoustic cloth window as a function of frequency. . . . . . . . . . . . . . . 27

3.6 2-D airfoil schematic . . . . . . . . . . . . . . . . . 28

3.7 2-D schematic drawings of flat plate [a] Straight, [b] Swatooth, [c] Slitted, and, [d] Sinusoidal TE extensions. . . . . . . . . . . . . . . . . 30

$3.8 \quad$ SPL in the starboard-side anechoic chamber $(1.4 \mathrm{~m}$ from the test-section center) as a function of flow speed in the empty test section. . . . . . . . 35

3.9 The overall sound pressure level of background noise as a function of freestream velocity. . . . . . . . . . . . . . . 36 
3.10 Comparison of A-weight sound pressure level of Carleton University wind tunnel to other acoustic facilities worldwide. . . . . . . . . . . 37

3.11 Measured SPL spectra for NACA-0012 airfoil and the same airfoil with sawtooth TE at free-stream velocities of (Left) $14 \mathrm{~m} / \mathrm{s}$ and (Right) $24 \mathrm{~m} / \mathrm{s}$ at Carleton University anechoic wind tunnel. The background noise spectra are also shown in this figure for comparison. . . . . . . . . . . . . . 38

3.12 Far-field acoustic spectra for the reference flat-plate and the flat-plate with trailing edge serrations, slitted TE, at freestream velocities of (Left) $14 \mathrm{~m} / \mathrm{s}$ and (Right) $24 \mathrm{~m} / \mathrm{s}$. The background noise spectra are also shown in this figure for comparison. . . . . . . . . . . . . . . . . 39

3.13 Normalized spectrum of noise produced by a low Mach number flow over a sawtooth TE: (a) from $|3|$ equation (17) ;(b) Matlab code.. . . . . . . . . 42

3.14 Normalized spectrum of noise produced by a low Mach number flow over a slitted TE: (a) from $|4| \mid$ equation (10); (b) Matlab Code. . . . . . . . . . 42

3.15 Normalized spectrum of noise produced by a low Mach number flow over a sinusoidal TE: (a) from $|3|$ Equation (5.6); (b) Matlab Code. . . . . . . . 43

$3.16 S P L$ plotted as a function of frequency for optimum optimized sawtooth

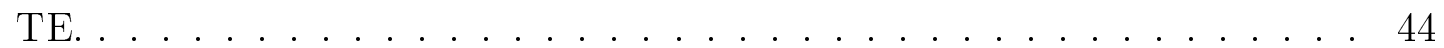

3.17 Normalized spectrum plotted as a function of frequency between $0.1 \mathrm{kHz}$ and $10 \mathrm{kHz}$ for $O A S P L_{\text {norm }}$ optimized slitted trailing edge profiles. . . . . 45

3.18 Normalized spectrum plotted as a function of frequency between $0.1 \mathrm{kHz}$ and $10 \mathrm{kHz}$ for $O A S P L_{\text {norm }}$ optimized sinusoidal trailing edge profiles. . . 46

3.19 The compression between the optimum serrations. . . . . . . . . . . . . . 47

3.20 Measured far-field noise spectrum showing a comparison between straight and the sawtooth, slitted and sinusoidal TE with airfoil and flat-plat at $0^{0}$ AoA and $\mathrm{U}=24 \mathrm{~m} / \mathrm{s} . \ldots \ldots \ldots \ldots \ldots$. . . . . . . . . . . 48 
3.21 Broadband noise reduction predicted by Howe (Dashed) and measured experimentally (Solid) for serration profiles SS-Saw-1, SS-Slit-1 and SS-Sinu-2 - Experimental data with airfoil and flat-plate at $0^{0}$ AoA and $\mathrm{U}=24 \mathrm{~m} / \mathrm{s} . \quad 49$

$4.1 \quad$ Flat-plate with serrated trailing-edge and the related local coordinates sys-

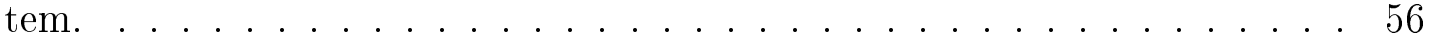

4.2 Normalized spectrum of noise produced by a low Mach number flow over a sawtooth TE: (top (a) and (b) ) from Lyu et al. |5|, equation (3.6) ;(bottom (a) and (b)) Matlab code. . . . . . . . . . . . . . . 63

4.3 Predicted non-dimensional far-field noise $S P L_{n o r m}$, (a), and corresponding noise reduction $\triangle S P L_{\text {norm }}$, (b), by different sawtooth serrations according to the optimization study at $\mathrm{U}=24 \mathrm{~m} / \mathrm{s}$. A positive $\triangle S P L_{n o r m}$ denotes noise reduction. . . . . . . . . . . . . . . . 66

4.4 Wind tunnel at Carleton University. . . . . . . . . . . . . . . . . . . 68

4.5 Flat-plate model with TE serrations. . . . . . . . . . . . . . . 69

4.6 2-D airfoil model with TE serrations. . . . . . . . . . . . . . . . 70

4.7 National Instruments PXIe-1071 DAQ system. . . . . . . . . . . . . . . . 71

4.8 Typical 4944-A microphone and its frequency response. . . . . . . . . . . . 72

4.9 Left: Acoustic calibrator. Right: sound pressure level meter. . . . . . . . . 72

4.10 The calibration result obtained for microphone 1. . . . . . . . . . . . . . 73

4.11 Experimental setup for the aeroacoustic test of the airfoil noise (top view). 74

4.12 Power spectrum density level at mid-span and $90^{\circ}$ above the trailing-edge of the: (a) flat-plate (b) NACA-0012 airfoil, computed with Lyu's model and compared with the experimental measurements. . . . . . . . . . 75

4.13 Comparison of the noise spectra between optimization predictions and experimental measurements at $24 \mathrm{~m} / \mathrm{s}$ and $0^{0}$ AoA for flat-plate sawtooth TE

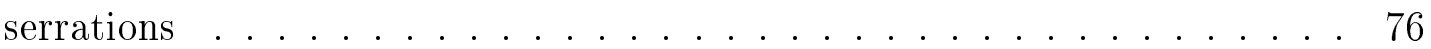


4.14 Comparison of the noise spectra between optimization predictions and experimental measurements at $24 \mathrm{~m} / \mathrm{s}$ and $0^{0}$ AoA for $\mathrm{NACA}-0012$ airfoil sawtooth TE serrations. . . . . . . . . . . . . . . . . . . 77

$5.1 \quad$ Schematic illustration of the segregated modeling domains. . . . . . . . . . 95

5.2 General serration configuration, not to scale. . . . . . . . . . . . . . 95

5.3 Computational mesh. . . . . . . . . . . . . . . . . . . 97

5.4 Instantaneous $y+$ distribution. . . . . . . . . . . . . . . 98

5.5 Boundary Conditions. . . . . . . . . . . . . . . . . 99

5.6 Wind tunnel configuration at Carleton University. . . . . . . . . . . . . . . 100

5.7 Cross section through the aeroacoustic test section and anechoic chamber as seen from above (left) and photograph of serrated TE airfoil in test section (right). . . . . . . . . . . . . . . . . 101

5.8 From left to right: NACA-0012 main body, TE of case E0, TE of case E1 and TE of case E2. . . . . . . . . . . . . . . . . . 102

$5.9 \quad C_{p}$ distribution on airfoil surface. . . . . . . . . . . . 104

$5.10 P_{R M S}$ distribution near airfoil TE. . . . . . . . . . . . . . . 104

5.11 Lift-coefficient history of C2.3 using two different meshes. . . . . . . . . . . 106

5.12 Lift-coefficient history for case C1.2, C2.1, C2.2 and C2.3. . . . . . . . . . 108

5.13 Instantaneous Q-Criterion colored by $\omega_{z}$. . . . . . . . . . . . . . 109

5.14 Instantaneous vorticity magnitude contours. . . . . . . . . . . . . . . 110

5.15 Instantaneous $\omega_{x}$ contours at $x / c=1 . \ldots \ldots \ldots$. . . . . . . . 111

5.16 Time domain noise signals propagated to receiver. . . . . . . . . . . . 113

5.17 Frequency domain noise signals of C1.2, C2.1, C2.2 and C2.3 . . . . . . . 113

5.18 Frequency domain noise signals of C2.3 using 2 meshes. . . . . . . . . . . . 114

5.19 Experimentally obtained SPL. . . . . . . . . . . . . . . 116 
6.1 Airfoil model and trailing-edges: Straight trailing-edge (Str.TE), and Saw1, Saw-2, Saw-3 and Saw-4. . . . . . . . . . . . . . . 123

6.2 Wind tunnel at Carleton University. . . . . . . . . . . . . . . . 124

6.3 Sketch of the experimental setup for the aeroacoustic measurements. . . . . 125

6.4 Region of Reynolds number, $R e_{c}$, and angle of attack, AoA, where tonal noise can be found for a NACA-0012 Airfoil. Adapted from Probsting et

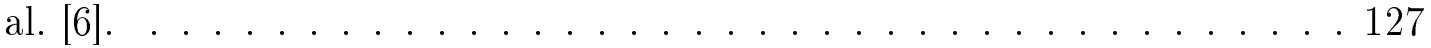

$6.5 S P L$ spectra radiated by the straight airfoil at $0^{0}$ AoA for various inflow velocities. . . . . . . . . . . . . . . . . 129

6.6 SPL spectra radiated by the straight airfoil for various AoA at a Reynolds number of $5.00 \times 10^{5}$. . . . . . . . . . . . . . . . 130

6.7 SPL measured at $\alpha=5^{0}$ AoA and $\mathrm{U}=14,18$, and $24 \mathrm{~m} / \mathrm{s}$ for; a) untripped airfoil ;b) tripped airfoil. . . . . . . . . . . . . . . . . 131

6.8 Effect of sawtooth trailing-edges on airfoil instability noise at an angle of

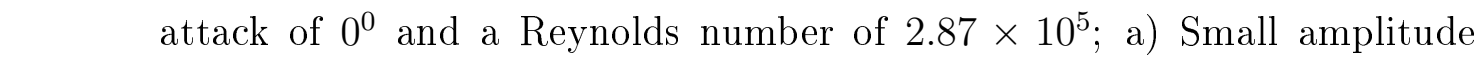
(Saw-1 and Saw-2); (b) large amplitude (Saw-3 and Saw-4). . . . . . . . . 132

6.9 Effect of sawtooth trailing-edges on airfoil instability noise at an angle of attack of $5^{0}$; a) $R e_{c}=3.71 \times 10^{5}$; b) $R e_{c}=5.00 \times 10^{5}$. . . . . . . 133

6.10 Effect of sawtooth trailing-edges on airfoil instability noise at an angle of attack of $10^{0}$ and; a) $R e_{c}=3.71 \times 10^{5}$; b) $R e_{c}=5.00 \times 10^{5}$. . . . . . . 134

6.11 Effect of sawtooth trailing-edges on airfoil instability noise at an angle of attack of $10^{0}$ and a Reynolds number of $5.00 \times 10^{5}$. . . . . . . . . 135

$7.1 \quad$ Wind tunnel upgrading. . . . . . . . . . . . . . . . . 145 


\section{List of Tables}

$3.1 \quad$ Optimization trials and associated design variable (DV) bounds for $(U=$

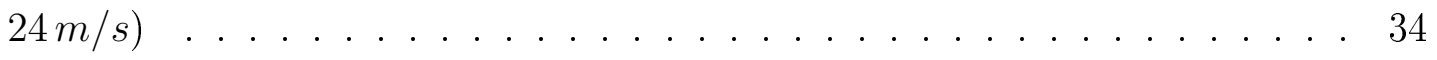

3.2 Optimized designs from each study and noise produced by each trailing

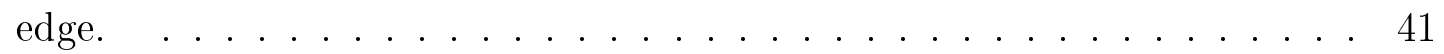

$4.1 \quad$ Optimization trials and associated design variable bounds $(U=24 \mathrm{~m} / \mathrm{s})$. . 62

4.2 The parameters used in the solution. . . . . . . . . . . . . . . . . 64

$4.3 \quad$ Optimized designs from sawtooth study and noise produced by their trailing-

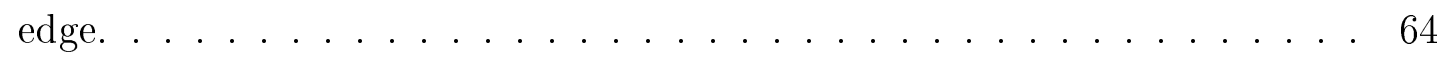

5.1 Geometric parameters of simulated airfoils. . . . . . . . . . . . . . . 94

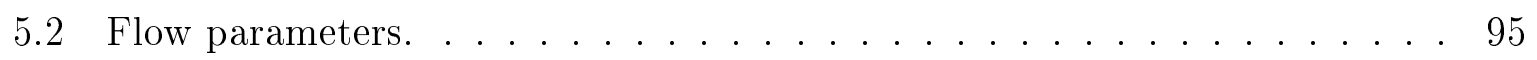

5.3 Mesh elements. . . . . . . . . . . . . . . . . . . 98

5.4 Experimental geometric parameters. . . . . . . . . . . . . . . 102

$6.1 \quad$ Design parameters of TE in the present study. . . . . . . . . . . . . . 123

A.1 Sources of systematic uncertainties with total bias uncertainty . . . . . . . 164

A.2 Uncertainties in noise level. . . . . . . . . . . . . . 165 


\section{List of Acronyms}

\begin{tabular}{ll}
\hline Acronym & Definition \\
\hline AoA & Angle of Attack \\
ANC & Active Noise Control \\
$B L$ & Boundary layer \\
CAA & Computational Aeroacoustics \\
DV & Design Variable \\
EPN & Effective Perceived Noise \\
OASPL & Overall Sound Pressure Level \\
PNC & Passive Noise Control \\
PSD & Power Spectral Density \\
PDE & Partial Defferential Equation \\
$S G S$ & Subgrid-scale \\
SPL & Sound Pressure Level \\
$T_{F T}$ & Through-flow time, $s$ \\
TBL & Turbulent Boundary Layer \\
\hline
\end{tabular}




\section{List of Symbols}

\begin{tabular}{|c|c|}
\hline Symbol & Description \\
\hline $\mathrm{c}$ & Chord length \\
\hline$c_{0}$ & Speed of sound \\
\hline$C_{m}$ & $\begin{array}{l}\text { Friction velocity and mean shear interaction } \\
\text { pressure constant }\end{array}$ \\
\hline$C_{p}$ & Pressure coefficient \\
\hline$E$ & Complex error function \\
\hline$f$ & frequency \\
\hline$G(x, y ; \omega)$ & Green's function \\
\hline$h$ & Half of root-to-tip length of serrations \\
\hline$h_{\min }, h_{\max }$ & Smallest and largest feasible serration amplitudes \\
\hline$h_{\text {opt }}$ & Optimum serration amplitude \\
\hline$i$ & Imaginary number $\sqrt{-1}$ \\
\hline $\mathbf{K}$ & Wavenumber vector \\
\hline$K_{1}$ & Streamwise wavenumber \\
\hline$K_{3}$ & Spanwise wavenumber \\
\hline$L$ & Length, $m$ \\
\hline$M_{0}$ & Mach number of uniform flow \\
\hline$N$ & $\begin{array}{l}(2 \mathrm{~N}+1) \text { denoting the number of periods of } \\
\text { serrations }\end{array}$ \\
\hline$P_{b}$ & Blocked pressure \\
\hline$P_{s}$ & Scattered pressure \\
\hline$P_{f}$ & Far field sound pressure \\
\hline
\end{tabular}


$Q_{n m} \quad$ Function defined for expressing wall pressure gust response

Q Second invariant of velocity gradient tensor

$R e_{c} \quad$ Reynolds number based on chord length

$s \quad$ Airfoil span, $m$

$S_{n m} \quad$ Function defined for expressing wall pressure gust response

$S_{p p} \quad$ PSD of far-field sound

$t \quad$ Time, $s$

$u_{\star} \quad$ Friction velocity in Chase's model

$u, v, w \quad$ Fluid velocities, $\mathrm{m} / \mathrm{s}$

$x_{1}, x_{2}, x_{3}$ Location of a far-field observer (Cartesian coordinate system)

$x_{1}, x_{2} \quad$ Design variables in the optimization test case

$y_{1}, y_{2}, y_{3} \quad$ Location of a point source in the vicinity of the trailing-edge

$Z \quad$ Objective function value

$\lambda \quad$ Sawtooth and sinusoidal serration width

$\lambda_{1}, \lambda_{2} \quad$ Slitted serration width and gap width

$\lambda_{\text {opt }} \quad$ Optimum wavelength serration width

$\delta \quad$ Turbulent boundary layer thickness

$\kappa \quad$ acoustic wavenumber $(\kappa=\omega / c)$

$k_{2 n} \quad$ Characteristic wavenumber of mode $n$, $k_{2 n}=k_{2}+2 n \pi / \lambda$

$k_{1} \quad$ Hydrodynamic wavenumber in chordwise direction $k_{1}=\omega / U_{c}$

$k_{2} \quad$ Hydrodynamic wavenumber in spabwise direction 


\begin{tabular}{|c|c|}
\hline $\mathcal{L}$ & Gust-response function \\
\hline$\beta$ & $\beta=\sqrt{1-M_{0}^{2}}$ \\
\hline$\chi$ & Dimensionless constant in Chase's model \\
\hline$\Gamma\left(y_{3}\right)$ & Serration profile function \\
\hline$\delta$ & Turbulent boundary layer thickness \\
\hline$\epsilon_{j}$ & $\begin{array}{l}\text { The chordwise coordinate of the starting point of } \\
j-t h \text { edge }\end{array}$ \\
\hline$\epsilon_{j+1}$ & $\begin{array}{l}\text { The chordwise coordinate of the ending point of } \\
j-t h \text { edge }\end{array}$ \\
\hline$\eta_{A m}$ & Wavenumber in $S_{n m}$ \\
\hline$\eta_{B m j}$ & Wavenumber in $S_{n m}$ \\
\hline$\kappa_{n j}$ & Wavenumber in $Q_{n m}$ \\
\hline$\varepsilon$ & Integrated source layer thickness constant \\
\hline$\epsilon$ & Machine epsilon \\
\hline$\theta$ & $\begin{array}{l}\text { Angle between the } x_{1} \text { axis and the far-field } \\
\text { observer (in the } x_{1}-x_{2} \text { plane) }\end{array}$ \\
\hline$i_{3}$ & Unit vector parallel to the airfoil span \\
\hline$j$ & $\begin{array}{l}\text { The } j-t h \text { edge of a single piece of sawtooth } \\
\text { serrations }\end{array}$ \\
\hline$\lambda$ & Wavelength of serrations \\
\hline$\lambda_{\min }, \lambda_{\max }$ & Smallest and largest feasible serration wavelength \\
\hline$\lambda_{o p t}$ & Optimum serration wavelength \\
\hline$\Psi$ & Non-dimensional trailing edge noise spectrum \\
\hline$\Psi_{0}$ & $\begin{array}{l}\text { Straight edge non-dimensional trailing edge noise } \\
\text { spectrum }\end{array}$ \\
\hline$\Psi_{\text {saw }}$ & $\begin{array}{l}\text { Sawtooth edge, non-dimensional trailing edge } \\
\text { noise spectrum }\end{array}$ \\
\hline
\end{tabular}




\begin{tabular}{ll}
$\Psi_{\text {slit }}$ & Sitted edge, non-dimensional trailing edge noise \\
& spectrum \\
$\Psi_{\text {sinu }}$ & Sinusoidal edge, non-dimensional trailing edge \\
& noise spectrum \\
$\omega$ & Angular frequency $\omega=2 \pi f$ \\
$\Pi$ & Wavenumber spectral density \\
$\rho_{0}$ & Fluid density \\
$\sigma_{j}$ & Slope of the $j-t h$ edge \\
$\sigma$ & Absolute value of the slope of a sawtooth edge \\
$\Theta_{n}$ & Fundamental order of the gust-response function \\
$\Theta_{n}^{(i)}$ & $i-t h$ order of the gust-response function \\
$\xi$ & Integral variable \\
$\varepsilon$ & Serration root bluntness, $m$ \\
$\mu$ & Dynamic viscosity, $N s / m^{2}$ \\
$\nu$ & Kinematic viscosity, $m^{2} / s$ \\
\hline & Density, $k g / m^{3}$
\end{tabular}




\section{Chapter 1}

\section{Introduction}

The science of aeroacoustics and aerodynamic noise has a 70-year history [7,8], which appeared as a result of the growing problem of noise pollution caused by technological advancements. James Lighthill $[9]$ identified aerodynamic turbulence as a source of sound in the early 1950s, setting a foundation for the investigation of a wide range of aeroacoustic issues that have become a source of major concern in civil and military aviation. The current study aims to contribute to the examination and reduction of aerodynamic airfoil self-noise, relevant in airplane wings, wind turbine blades, jet engine and industrial cooling fans.

\subsection{Motivation}

The past few decades have seen an expanding network of transportation and the use of air traffic. The development of civil aviation has caused changes in our daily lives and has become a fundamental pillar of our global society. The aircraft industry, for example, is a growing market with an increase of $5 \%$ per year in civil aviation and about $6 \%$ in the military [10. Moreover, the number of passengers has a trend of doubling every $15-20$ years, which is equivalent to an annual growth rate of about "4-6\%" 11, 12. Nonetheless, the persistent problem of noise is a serious impediment to a smooth expansion of the civil aviation sector. Noise pollution is a major concern for passengers on the aircraft and for 
people living in close proximity to airports. Aircraft noise can affect communities of all sizes, from large cities with busy international airports to rural settings where private airstrips are sometimes found. Throughout the $20^{\text {th }}$ century, aircraft noise was considered a problem primarily because it is a nuisance [13|. Researchers started studying the effects of aircraft noise on human health around the turn of the century [14 17]. There have been studies on sickness, annoyance, and learning disabilities. According to the findings of these studies, regular and/or extended exposure to aircraft noise can have substantial, negative health consequences for humans. Aircraft noise has been linked to serious health impacts such as stress, hypertension, sleep disturbances, ischemic heart disease, hearing damage, and discomfort, among others [18,19]. According to reports from the UK Civil Aviation Authority [20] and the UK National Noise Attitude Survey [18], aircraft noise affects more than 700,000 individuals in the Heathrow Airport area alone, and more than one million people across the UK. According to the British Medical Journal study, there is a "statistically significant association between aircraft noise and the risk of hospitalization for cardiovascular diseases." [16]. Research presented at the American Heart Association's Scientific Sessions 2018 suggests that chronic exposure to high levels of noise can increase the risk of heart attack and stroke. Additionally, aviation noise has an impact on not just individuals on the ground, but also flight crews and passengers on board. For example, noise levels inside the cabin of an Airbus A321 during cruise have shown to be excessive, averaging around $78 \mathrm{~dB}$ [21], which is similar in magnitude to highway traffic or a washing machine. As a result, aircraft designers and airlines are promoting lower noise levels inside and outside new aircraft.

In this context, health concerns caused by airplane noise, as well as a associated social, economic, and environmental challenges, are a big concern for today's aviation sector, and the situation is only getting worse. In 2014, there were over 3,864 airports worldwide with regular commercial flights, serving over 33 million passengers [22. It is expected that aviation traffic will at least double in the next 20 years $[23 \mid$. Furthermore, the commercial 
aviation sector is shifting toward larger and faster aircraft, which will result in an increase in the amount of noise produced per aircraft. As a direct result of increasing both flight volume and noise per aircraft, existing regulations concerning noise limits continue to become more strict [24], forcing aircraft to comply or retire. These factors establish a clear and definite motivation for aircraft designs that are quieter. The most effective method of noise reduction is the direct elimination of noise at the sources; therefore, whenever possible, noise-source reduction should be considered. It is undeniable that aircraft noise is still a significant and developing concern; yet, improvements in the design of low-noise systems and components have been developed over time. The Effective Perceived Noise (EPN) for aircraft has dropped by approximately $20 \mathrm{~dB}$ since the mid 1900s [25]. While this is a significant improvement, nearly all of the reduction is attributed to the engine's improved aeroacoustic performance, with less emphasis on airframe noise [26]. While the engines are a major source of noise during take-off, during approach and landing the engines are throttled down significantly. During these two phases of flight, the major source of noise is caused by the airflow interacting with the airframe. One of the main sources of airframe noise is produced by the trailing-edge (TE) of surfaces that are exposed to the mainstream of airflow 25,27]. Although there has been some research in this area, the industry has been hesitant to use superior TE noise reduction methods. Reasons for the lack of adoption include the limitation in available design tools and a low cost-toimprovement ratio for current technologies. The work involved in this thesis aims to give a contribution to this field. Obtained results aim to advance the field towards industry acceptance and application of TE noise reduction technology.

\subsection{Objectives}

The main goal of this thesis is to advance the state of the art of research in TE design for noise reduction. This is achieved through numerical studies using semi-empirical models, and experimental testing in a wind tunnel at Carleton University. Different TE 
configurations of NACA-0012 airfoil and flat-plate serrations were investigated to reduce airfoil self-noise. To achieve this goal, the following objectives are addressed in this thesis:

1. Design and characterize an aeroacoustic wind tunnel section suitable for aeroacoustic research. The test section was used to test optimum airfoil and flat-plate TE serrations in order to validate the numerical performance predictions and to highlight any discrepancies. Wind tunnel experiments provide further insight into any differences between numerical and experimental analyses, emphasizing the importance of wind tunnel testing as part of the design process.

2. Quantify the level of noise attenuation in the performance of the aeroacoustics test section, and study the noise reduction by optimizing the airfoil TE geometry.

3. Create a framework for aeroacoustic numerical optimization of TE serrations as a tool for designing quieter structures.

4. Investigate theoretically optimum geometries of serrated TE extensions, and identify conditions of optimality through a series of optimization studies.

5. Implement, manufacture, and test the optimized serration TE designs to confirm TE optimization results.

6. Improve the concept of flat-plate and non-flat plate serrations, reduce Turbulent Boundary Layer (TBL)-TE noise and simultaneously achieve noise reductions when compared to a straight TE airfoil. It is the author anticipated that innovative solutions will rekindle interest for further research into this subject.

7. Provide a clearer picture of the flow physics over and past a serrated TE. The investigation of flow parameters is of tremendous interest since they are directly related to noise generation and radiation. The mechanisms involved have not yet been fully explored or understood. 


\subsection{Contributions}

The research contributions accomplished by the author during the course of this research are listed as follows:

1 - (Chapter 3) Design, installation, and testing of an aeroacoustic wind tunnel test section. The new concept involved the construction and installation of a test section with walls formed from tensioned cloth screens embedded in an anechoic chamber. This design has the potential to eliminate the need for a jet collector/catcher and reduced interference effects. The concept was tested in the medium-speed wind tunnel at Carleton University. The existing wind tunnel test section was modified to incorporate prototype acoustic treatment, two acoustic transparency sheet side-walls, and surrounding acoustic enclosures. Background noise measurements over the whole speed range of the facility showed that the acoustic treatment was effective in reducing noise levels. A NACA-0012 airfoil and flat-plate model were placed inside the new test section to perform aeroacoustic measurements for proof-of-concept.

2 - The reduction of TBL-TE noise using flat-plate type TE serrations predicted by semi-empirical models was examined through optimization studies. Different serration shapes were investigated. The optimization studies were carried out using the optimization MATLAB code developed by the author for this thesis. Throughout the work included in this part of the thesis, a number of contributions have been made to the state of the art as follows:

2.1 - (Chapter 3) The numerical optimization program was developed. The validity of Howe's semi-empirical TBL-TE noise model for a serrated TE is discussed. By comparing

the numerical results with the experimental measurements from the medium-speed wind tunnel at Carleton University, it was established that Howe's model is an appropriate noise prediction tool for the work in this thesis. A detailed numerical optimization is conducted using Howe's model for the sound reduction and overall sound pressure level (OASPL) 
achievable using sawtooth, slitted, and sinusoidal serrations at the TE were performed and compared to a straight TE configuration. Prototypes of the optimum serrations were manufactured and tested.

2.2 - (Chapter 4) A formal numerical optimization study to find the optimal sawtooth shape for TE serrations that maximized noise reduction was conducted. While previous optimization studies have used (by the author) the original Howe's model [22], the current approach implements Lyu's model [23], which is an improved and more accurate model. The far-field sound pressure emitted from a NACA-0012 airfoil was obtained in wind tunnel. Four sawtooth TE geometries were tested on both the airfoil and a flat-plate for comparison. Results from the optimization study were then compared with wind tunnel measurements.

3 - (Chapter 3 \& Chapter 4) A framework for the numerical optimization of noise reducing TE serrations was developed. The framework includes an approach for optimizing TBL-TE noise reduction, which uses the recent semi-empirical noise models (Howe first and then Lyu) to calculate the objective function values. Previous efforts towards TBL-TE noise reduction optimization were limited to experimental trial and error of a finite number of geometry variations and elaborate numerical simulations employing computational aeroacoustics (CAA), both of which are time consuming and costly. The optimization framework established in this thesis provides a means of conducting highly efficient TBL-TE noise optimization studies.

4 - (Chapter 3 \& Chapter 4) By creating a framework for the numerical optimization that allows the user to generate an optimal airfoil/blade TE for a given operating condition (e.g. landing flight or wind turbine), it provides the industry another tool to improve aircraft passengers comfort, and the quality of life for people who live around the airport or near wind turbine farms when designing a new aircraft/wind turbine with that are 
quieter. Currently, no such software exists that can optimize the airfoil TE parameters of an aircraft or wind turbine to best reduce the noise from the TBL.

5 - (Chapter 3 \& Chapter 4) The program (a framework for the numerical optimization) created in the present study calculates the optimal TE serrations providing a method to reduce currently costly acoustic flight and wind tunnel testing by using a more inexpensive and simpler approach on the ground in an acoustically treated environment. This allows acoustic testing to be performed prior to the final aircraft TE wings being manufactured, which may result in changes to the design being made early in the design before significant costs are committed to a project.

6 - (Chapter 3 \& Chapter 4) Reducing aircraft or wind turbine noise used to be done at the end of the design stage whereby it is measured and improvements are implemented to best help. This software/program allows for the evaluation to happen early in the preliminary design phase, considering noise to be a design parameter right at the beginning of the design stage.

7- (Chapter 5) Successful implementation of ELES in tandem with the FW-H aeroacoutic analogy to predict flow-field around NACA-0012 airfoils equipped with different bio-inspired noise-attenuating TE designs. Far-field noise is also estimated at a fraction of the computational cost needed for full LES or DNS resolution.

8 - (Chapter 6) The character and level of TE self-noise are known to be highly sensitive to Reynolds number, angle of attack, and airfoil geometry. An extensive study is conducted on the instability noise characteristics from non-flat plate serrations at three different angles of attack $(\alpha) 0^{0}, 5^{0}$ and $10^{0}$, across low to moderate Reynolds numbers between $2.87 \times 10^{5}, 3.71 \times 10^{5}$, and $5.00 \times 10^{5}$. 


\subsection{Outline}

The current thesis is an integrated work that incorporates six papers which are either published or under review. This section provides an outline of the remaining chapters and papers included in this thesis.

Chapter 2: This chapter contains a literature review of related works which provides the reader with the necessary fundamentals and background information required for the understanding and interpretation of airfoil TE noise and airfoil self-noise reduction. Furthermore, it will highlight major studies that have been performed in numerical optimization and experimental testing on the design of airfoil and flat-plate TE serrations.

Chapter 3: The paper published in Canadian Acoustics Journal in Sep. 2020 [28, titled "Optimization and Testing of Flat-Plate Trailing-Edge Serration Geometry for Reducing Airfoil Self-Noise."

Conference paper published in the Proceedings of the 2019 Acoustic Week Canada in Oct. 2019 [29], titled "Development and Testing of an Aeroacoustic Wind Tunnel Test Section."

Conference paper published in the Proceedings of the 27th International Congress on Sound and Vibration (ICSV27) in Jul. 2021, titled "Numerical Optimization and Wind Tunnel Testing of Trailing Edge Serrations for Reducing Airfoil Self-Noise."

Chapter 4: Publication submitted and under review, titled "Aeroacoustic Design Optimization and Experimental Investigation of Sawtooth TEs for Maximized Noise Reduction."

Chapter 5: Publication submitted and under review, titled "ELES and Tonal Noise Prediction of Serrated-TE Airfoils."

Chapter 6: Publication submitted and under review, titled "Experimental Investigation of NACA-0012 Airfoil Instability Noise with Sawtooth Trailing Edges."

Chapter 7: This chapter contains the conclusions of this thesis, as well as recommendations for future work. 
Chapter 2

\section{Background and Literature Review}

In this chapter, an overview of the relevant research in TE noise reduction is presented. The focus of this review is on the mechanisms responsible for the airfoil TE noise, methods of TE noise reduction, and methods of TE noise prediction.

\subsection{Airfoil Self-Noise Mechanisms}

Airfoil self-noise is the noise radiation resulting from the interaction of the flow structures with the airfoil TE [1]. The first attempts to identify the source of TE noise were carried out in 1959 by Powell [30|. In the following years the topic was researched extensively with works by Ffowcs Williams and Hall (1970), Chase (1975), Paterson (1976), Howe (1978), and Brooks (1989) amongst others. During the 1980s, in an effort to understand and predict the mechanisms of airfoil self-noise, a series of research studies were conducted at, and supported by, the NASA Langely Research Center [1]. As shown by Brooks et al. [1]( Figure 2.1) the noise radiation can be tonal due to instabilities developing in a laminar boundary layer or broadband if the boundary layer is turbulent and the boundary layer flow remains attached. The nature of the self-noise spectrum is dependent on which of the five mechanisms is dominant and can be defined as :

(a) . Laminar boundary layer - vortex shedding noise (LBL-VS)

The LBL-VS noise is tonal. The laminar flow regions extend to the TE at relatively 
low-to-moderate Reynolds numbers. The noise mechanism is activated when the laminar instabilities develop on the aerodynamic surface, causing flow fluctuations, resulting in tonal noise. Tonal noise results from the coupling of an upstream moving acoustic wave with the instabilities. As a result of the instabilities stated above, separation bubbles might form, causing noise. When instabilities are induced by the acoustic field, high amounts of noise might result, and vice versa [31].

(b) and (e) . Separation/Stalled flow noise (S/SF)

The angle of attack, AoA, has a big impact on this noise mechanism. Stall conditions occur at a certain point when the angle of attack increases, generating a significant amount of unstable flow around the airfoil and resulting noise.

\section{(c) . Trailing-edge bluntness-vortex shedding noise (TEB-VS)}

In the TE zone, pressure fluctuations are caused by alternating vortices. The noise mechanism is basis on vortex shedding type (e.g. Von Karman vortices) from the TE . The bluntness, form of the edge, and Reynolds number all influence the shedding of vortices from the TE as depicted in Figure 2.1.

\section{(d) . Tip vortex formation noise}

The difference in pressure between the suction and the pressure sides causes a vortex to form at the blade's tip. This vortex interacts with the tip surface to generate noise, which is similar to the turbulent boundary layer-trailing edge noise mechanism [1]. The convective velocity of the vortex and its strength are thought to have the largest impact on tip vortex noise, which is broadband in nature 31 .

\section{(f) . Turbulent boundary layer-trailing edge (TBL-TE) noise}

Boundary layers form over the surface of the airfoil, and at a given chord-wise location, the flow transitions from laminar to turbulent. When a turbulent boundary layer develops over the surface, the eddies that compose it are inefficient sound sources. The noise level of turbulence convecting along an airfoil is amplified by the TE of the airfoil. The convection speed of the eddies and the form of the boundary layer turbulence near to the edge have 
the greatest impact on TE noise. TBL-TE noise can be created by any surface with a TE that is exposed to flow conditions that create an incident turbulent boundary layer.

(a)

Laminer boundary layer

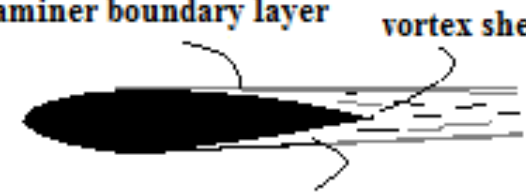

Instability waves

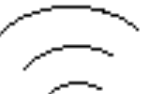

(b)

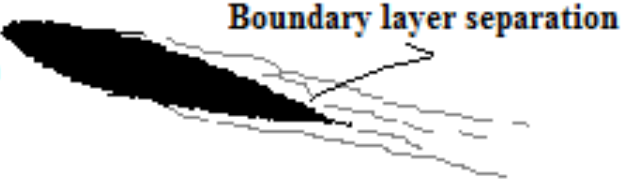

(c)

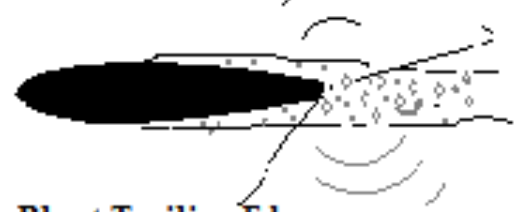

Blunt Trailing Edge
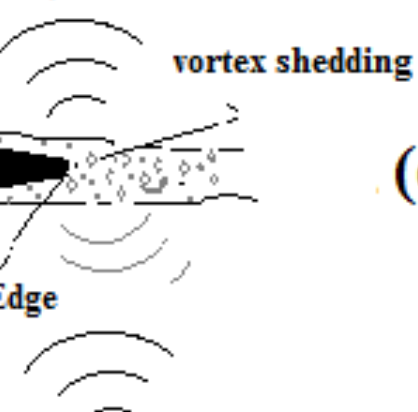

(e)

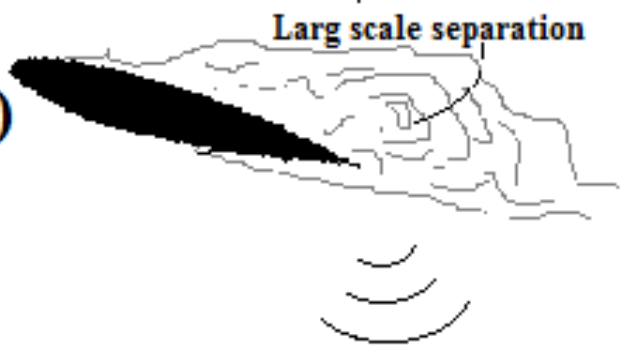

(d)

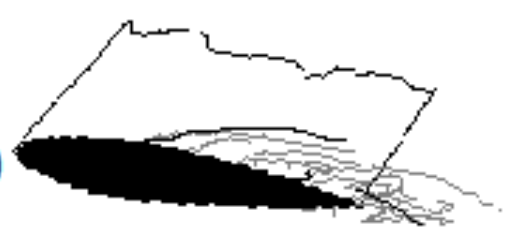

Tip vortex

sound wave emitted

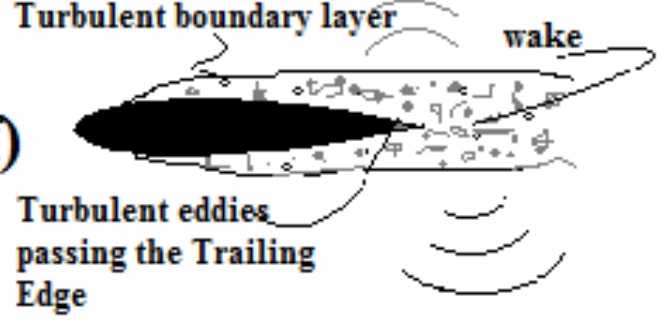

Figure 2.1: Airfoil self-noise mechanisms as defined by Brooks et al. |1].

There has been a significant amount of research conducted to investigate TBL-TE noise including experimental testing [1,32, 37], numerical simulations [4,38, 41], and analytical based noise modeling $3,36,42,48]$. The present work investigates available analyticalbased TBL-TE noise prediction methods and compares the results with experimental work.

\subsection{Airfoil Trailing-Edge Noise Reduction}

During the last several decades, different methods of reducing TBL-TE noise have been 
studied. The objective, in all methods, is to reduce or minimize the acoustic impedance discontinuity between the airfoil surface and free space. The mechanism for this reduction occurs differently for each method; however, they can be generally classified as either Passive Noise Control (PNC) methods or Active Noise Control (ANC) methods [49]. PNC methods reduce noise through a fixed-state interaction with the fluid domain in which the noise is produced and/or propagating. Noise reduction methods that are passive in nature include TE serrations, TE brush extensions, aeroacoustically designed airfoil profiles, increased material porosity near the TE, flexible TE, and various combinations of these 49. ANC is defined as a method of reducing noise using a control system to produce a noise-canceling response signal [30|. In general, ANC systems employ a senseanalyze-respond format where an undesirable event is sensed, a response is generated and then initiated to reduce or cancel noise. Active methods investigated include TE blowing/suction and responsive control of TE cross-sectional profile.

Each of the methods listed above have shown different levels of success in TBL-TE noise reduction. ANC methods tend to perform well to reduce the low frequency, tonal noise, while the opposite is true for PNC methods [50]. In addition to aeroacoustic performance, however, other considerations, relevant to the aviation industry, should be evaluated when comparing methods. Additional concerns include, for example, cost of maintenance, and added weight. Considering other non-acoustic factors will help to ensure the development of designs that have a high chance of industry acceptance. From this perspective, passive methods are, in general, more beneficial than active ones for typical aerospace applications.

\subsubsection{Biomimetics on Serration Design}

Researchers often look to many different sources for inspiration, with nature being a popular influence for modern innovations. Biomimetics is defined as the study of biological creatures structure and function as models for reverse engineering materials and products. In the context of this study, the silent flight of the owl was first investigated 
in 1934 by Graham [2], in which he identifies three specific attributes, which are directly related with the owl's impressive aeroacoustic performance. Graham observed that the Owl has developed: (1) wavy comb-type leading edges (or serrations); (2) fringed feather edges at the wing's TE; (3) velvety upper surface of the wing and a downy lower surface. At the time of discovery, Graham could not explain exactly how, or in what proportion, these three attributes contributed to the owl's silent flight. In the early 1970s, Graham's attributes were experimentally investigated by Kroeger [51] as part of a program researching novel designs for quieter aircraft. The study involved measuring the owl's flight noise both before and after the modification/removal of each of these attributes.

By comparing Graham's attributes to the literature on TBL-TE noise reduction methods, it is apparent that the two are closely linked. Whether or not these attributes have directly inspired researchers, they are highly relevant in all works regarding TBL-TE noise minimization. Of specific importance to the work in this thesis is attribute (2), the TE fringes, which are shown in Figure 2.2. The TE fringes create a smooth transition from the wing surface to the free stream, thereby interfering with the acoustic scattering process at the TE, typically observed [52]. Specifically, flat-plate serrated TE extensions simulate the geometry of the fringe at the TE of an Owl's wing, However, 2D representation is not an exact replication of an Owl's TE fringe as it does not account for certain features such as elasticity. 

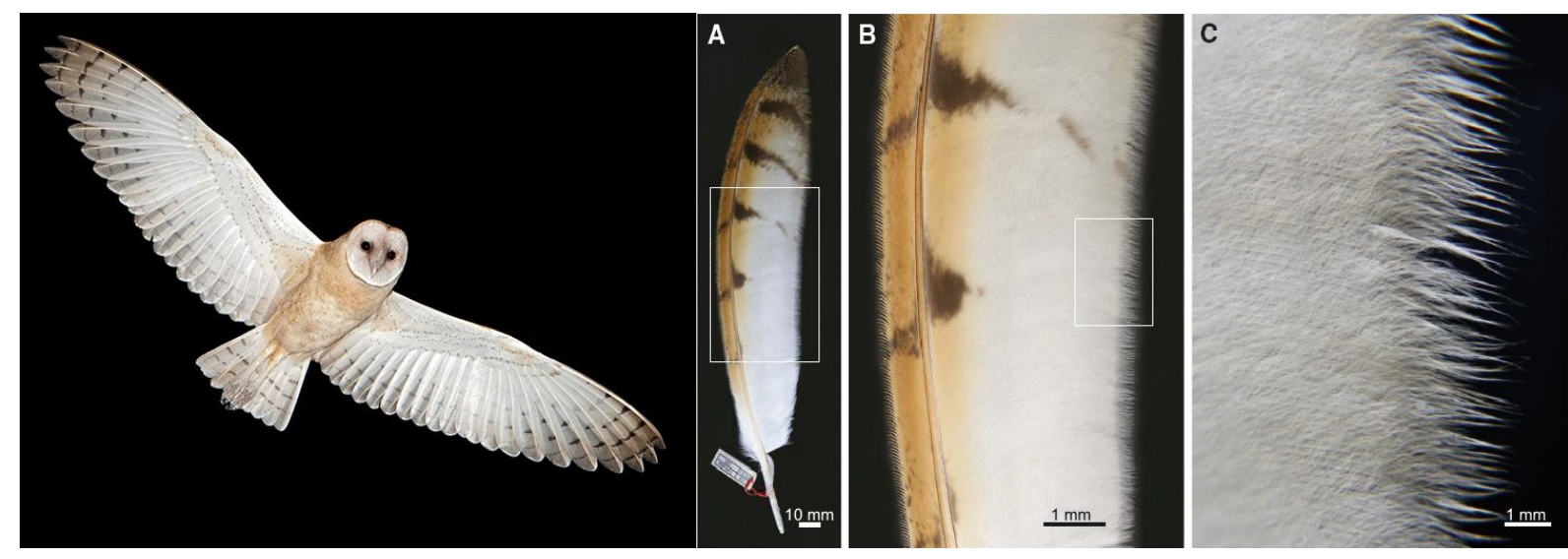

Figure 2.2: Structure of the Owl's wing in different magnifications showing the structure of the TE fringe (from Bachmann et al. [2|).

Bachmann and Wagner [52] state that the fringes are so pliant that they are freefloating in the flow. The effect that this feature has on the noise reduction performance has not been commented on in the literature, and no noise prediction methods currently exist that account for non-stiff TE serrations. Additionally, in a study of noise reduction using TE brush extensions, Herr [53] found that, while brush fiber flexibility is generally beneficial, it is not a prerequisite to obtaining noise reduction.

\subsection{Prediction Models of Serrated TE Noise}

The first observed prediction examination of low Mach number TE noise was carried out by Powell in 1959 [54]. Powell's analysis used similarity arguments to estimate the strength of dipole sources that were assumed to exist on the surface, near the TE. Additional TE noise modeling methods and theories, however, did not emerge until over a decade later. The contributions to TE noise research during this time were numerous (see Reference [55] for a detailed review), and notable developments were made by Ffowcs Williams and Hall [56], Chase [32], and Amiet [33]. The first of these additional noise models were derived by Ffowcs Williams and Hall [56] using Lighthill's acoustic analogy. Their model gives the radiated noise from a turbulent flow convecting past a semi-infinite, rigid plate with a zero thickness and at a zero angle of attack. The Ffowcs 
Williams and Hall model [56] was able to predict the shape of the radiation field, and the effects of flight relative to an observer's position, which were both improvements over Powell's model [54]. Several years later, Chase [34 produced his model using diffraction theory instead of Lighthill's acoustic analogy. Similar to the Ffowcs Williams and Hall model [56], Chase's model could predict the radiated field shape and flight effects; however, it had the added benefit of not having to consider the Lighthill sources explicitly. Instead, Chase quantified the sources using a turbulent pressure spectrum model, which is based on experimental data.

After considering the aeroacoustic performance observed experimentally, and the performance predicted from theory, one of the most promising methods is the use of TE serrations. Using serrations for airfoil noise reduction has initially been studied as an airfoil leading edge modification. In 1980, Fink and Bailey 57 published acoustic wind tunnel results of several different airframe reduction concepts; TE serrations being one of them. More than a decade later, M. S. Howe [3 published his semi-empirical model for predicting the TBL-TE noise produced by a serrated TE. Howe's model is an extension of his model for TBL-TE noise produced by an unmodified, straight TE [55], and is formulated such that a derivation of the model for any serration geometry can be obtained. A more detailed explanation of the derivation of Howe's model is given in Chapter 3 of this thesis. Howe first published the model for a sinusoidal serration geometry [35], and shortly after, for a sawtooth serration geometry [3]. In more recent years, Howe's model has been modified by other researchers for additional geometries including slits [37], and various superimposed combinations of the sinusoidal, sawtooth and slit serrations [4].

Up until very recently, Howe's model was the only method for predicting serrated TBL-TE noise without obtaining experimental results or conducting numerical simulation studies, which can both be very costly and time-consuming. In 2016, Lyu et al. 38 proposed a new approach to the Howe (1991) model that takes into account the hydrodynamic interaction between adjacent serrations, yielding findings that are more in 
agreement with noise prediction levels found in wind tunnel measurements. A more detailed overview of Lyu's model is given in Chapter 4. Howe's and Lyu's models are used in this work to compare with measurements taken in the Carleton University mediumspeed wind tunnel for the TE noise of the baseline and serrated NACA-0012 airfoil and a serrated flat-plate.

\subsection{Design Optimization for TE Noise Reduction}

Aircraft noise and associated design challenges cannot be isolated from other competing factors that influence the design and production of aircraft in today's aviation industry. For example, a complete and thorough optimization study of a certain aircraft design must consider the weight, effects of fuel efficiency, passenger comfort, safety and the frequency of maintenance, etc., in addition to noise production. Fast and efficient mathematical methods are therefore desirable for the prediction of TBL-TE noise, used as the objective function value in an optimization study [25].

To the author's knowledge, little work has been done in numerical optimization of the serrated TE shape for noise reduction. However, there has been some attention directed towards optimizing the cross-sectional shape of an airfoil to minimize TE noise, and in some cases, to improve the aerodynamic performance. Some of the initial work in searching for an acoustically optimized airfoil was conducted as part of the European-based wind turbine project, DATA [39]. This project did not employ computational optimization methods, but instead took the approach of identifying the sensitivity of TBL-TE noise to key flow parameters. Using the sensitivity information, several airfoil designs were then manufactured and tested. The test results revealed that the new designs produced less TBL-TE noise than the original airfoils, therefore, confirming that TE optimization can produce improved designs $[40]$.

Early studies that used computational optimization methods to reduce airfoil selfnoise only incorporated noise as an additional/secondary consideration for defining an 
objective function that describes an airfoil's overall performance 41. In the early 2000s, several studies, which emphasized optimizing airfoils for noise reduction, were published by Marsden et al. 42,44 and Lutz et al. 45]. The work of both sets of authors was based on defining the flow domain around the TE by numerically solving the NavierStokes equations, which can be computationally heavy. Marsden used the large eddy simulation method, while Lutz et al. 45] chose the Reynolds averaged Navier-Stokes method. The properties of the flow field near the TE were then used in acoustic models to quantify the noise produced by the TE, for use in the objective function. For the optimization method, Marsden et al. [42 used a gradient-based algorithm, called the method of "incomplete sensitivities", which is similar to a steepest descent method except that the large eddy simulation is solved only once per derivative calculation. Calculation of the derivative is done by approximating the perturbed objective function values to be used in the finite difference equation. Instead of a gradient-based algorithm, Lutz et al. 45 used a derivative-free, genetic algorithm for the optimization.

\subsection{Previous Experimental Investigations of the TE Noise}

In view of the growing aircraft industry in the early 1990s and later of the development of wind farms, researchers started addressing the airfoil TE noise problem using passive treatments such as serrations, brushes and porous TE. As presented by Dassen et al. 46 in 1996, Parchen et al. 47] in 1999 and Oerlemans et al. 48 in 2009, serrated edges have been used as means of reducing TE self-noise in airfoil wind tunnel tests and wind turbine applications. All of these studies have consistently shown noise reductions of up to $5 \mathrm{~dB}$ over broad frequency bands but an increase of noise at higher frequencies.

In 1996, Dassen et al. [46] experimentally investigated airfoil serrations trailing-edge noise reductions. Dassen et al. [46] tested various configurations of serrated TE, six flatplates and eight airfoils, in a wind tunnel. The experiments revealed that the serrated airfoils lead to noise reductions of up to $8 \mathrm{~dB}$, while the serrated flat-plate showed reduc- 
tions of up to $10 \mathrm{~dB}$ from 1 to $6 \mathrm{kHz}$. The noise increased at higher frequencies and no significant effect greater than $2 \mathrm{~dB}$ was reported.

In 1999, Parchen et al. 47] compared experimental results to Howe's theory using serrated TEs [35, 36]. Serrations were applied to wind turbines at either the scale of a wind tunnel and at full scale. Noise reductions of about $6 \mathrm{~dB}$ were obtained. When compared to measured values, Howe's theoretical model anticipated a substantially higher noise reduction.

In 2005, Herr [58] introduced various configurations of brushes in airfoil trailing-edges. In wind tunnel, various fiber diameters, separation distances between fibers, and brush lengths were examined. Over wide frequency ranges, noise reductions up to10 dB were recorded, due to the suppression of vortex shedding from the blunt baseline trailing-edge. The thickest trailing-edge brush provided the greatest noise reduction. This reduction, according to Herr [58], might be due to viscous damping of unsteady turbulent velocities in the brush zone.

In 2009, Oerlemans et al. 48 examined the use of serrations and airfoil shape optimization to minimize trailing-edge noise. Experiments were carried out in an open-jet wind tunnel and on a $94 \mathrm{~m}$ diameter three-bladed wind turbine. Due to its widespread use in current wind turbines, a NACA-6418 airfoil type was chosen as the reference blade. One blade was serrated along the span for TE noise reduction, one blade was optimized for aerodynamics, and the last blade was left untreated and utilized as the reference blade. Serrations showed reductions of up to $3 \mathrm{~dB}$. The optimized airfoil reduced overall TE noise by $3.2 \mathrm{~dB}$, with a maximum noise reduction of $5 \mathrm{~dB}$ up to $1 \mathrm{kHz}$.

More recently, experimental research with airfoil trailing-edge was conducted by Gruber [59]. Gruber focused on sawtooth serrations, conducting an experimental research in which thirty-seven sawtooth $\mathrm{TE}$ serration were tested at the wind tunnel for noise reduction at varied flow velocities and angle of attack. The slitted sawtooth TE and the leading-edge serrations were used to achieve noise reductions of up to $8.5 \mathrm{~dB}$. 
Chapter 3

\title{
Optimization and Testing of Flat-Plate
}

\section{Trailing-Edge Serration Geometry for}

\section{Reducing Airfoil Self-Noise}

\begin{abstract}
With an expanding network of transportation and the use of air traffic, noise radiated from civil aircraft during take-off and landing have become a major concern to communities nearby airports. The objective of this work is to investigate the reduction of Turbulent Boundary Layer-Trailing Edge (TBL-TE) interaction noise. In order to test the concept of noise reduction, the required experimental setup is designed and created. The first part of this study focuses on developing an aeroacoustic wind tunnel test section at Carleton University. The test section walls have been acoustically treated to simulate an acoustically far-field environment with forwarding flight. The two sides of the wind tunnel test section are fitted with anechoic chambers and lined with acoustic transparency tensioned cloth screens which act as an interface between the test section and the anechoic chambers to provide a smooth flow surface while eliminating the need for a jet catcher and reducing
\end{abstract}


interference effects. The ability of the trailing edge serrations to reduce TBL-TE noise is first analyzed through numerical optimization study, and trailing edges are after tested in a wind tunnel. Three different serration geometries are investigated. The noise spectra were modeled using Howe semi-empirical model for a semi-infinite flat plate, at zero angles of attack and low Mach number. NACA-0012 airfoil and flat-plate trailing edges are analyzed and tested. The results of the optimization studies are used to examine the influence of serration design parameters. It is shown that the sawtooth serrated trailing edges yield greater noise reductions than slitted and sinusoidal serrated trailing edges. Experimental and optimization results are then compared. It is concluded that numerical and experimental results are very suggestive that optimized serrated trailing edge configurations can yield less TBL-TE noise compared to the traditional straight-trailing edge configuration.

\subsection{Introduction}

The noise produced at and near airports is a major source of noise to surrounding communities, airport employees and, people that travel frequently. Aircraft noise has always been undesirable, and in recent years several studies have been released that make a significant connection between exposure to aircraft noise and an increased risk of cardiovascular diseases 14, 16, 17. The problem of aircraft noise then becomes much larger when considering trends in the aerospace industry that include increasing aircraft size and speed as well as major increases in the volume of air traffic 23. The two segments of an aircraft's flight where the most amount of noise is radiated to nearby communities are the take-off and landing phases. During the landing phase, while the engine throttle is turned down significantly, an important source of noise arises from the turbulent boundary layers (TBL) flow-structures convecting downstream across solid surfaces and interacting 
with the trailing edges. This mechanism of noise generation is known as the turbulent boundary layer-trailing edge (TBL-TE) noise, and its frequency spectrum is broadband in nature. TBL-TE noise is also a major source of noise in other applications, such as from wind turbines where exposure to nearby communities has been a complaint. With the growing global adaptation of alternative, environmentally friendly sources of energy, the implementation of wind turbine farms is quickly increasing. Consequently, the noise produced by wind turbines has become a significant issue in rural communities across the world where the wind farms are most often located [50]. Airframe noise research suggests that further research should be focused on noise generation mechanisms and noise reduction techniques. One promising method of reducing TBL-TE noise is by the addition of a flat-plate, serrated trailing edge extensions. This method of noise reduction has long been considered viable but has received increased attention in the last decade. The research has included experimental, theoretical and numerical studies $3,53,60,61$ that have all shown the ability of trailing edge serrations to reduce TBL-TE noise. The amount of noise reduction and frequency ranges in which noise attenuation occurs varies between studies; however, it is generally agreed that for at least some part of the frequency spectrum, trailing edge serrations can produce TBL-TE noise reductions of a significant level [62]. Optimization studies have previously been conducted to reduce TBL-TE noise 44, 63]; however, they have mainly focused on altering the airfoil profile at and near the trailing edge. These studies were able to show that new, lower-noise designs could be generated through numerical optimization processes by using a semi-empirical model of noise prediction. General trends have been inferred from research involving the prediction of TBL-TE noise from serrated trailing edges. The flow phenomena around aircraft components are complex 6, 64, 65], thus the noise generation mechanisms are difficult to be understood. Although the CFD combined with the acoustic analogy method has been widely applied in airframe noise prediction 66, 67, this method is costly and still lack of enough accuracy due to the limit of grid size, especially at high-frequency range. However, wind tunnel ex- 
periments can lead us to a better understanding of the noise mechanisms, and the results can also be used as benchmarks for the validation of numerical methods. Aeroacoustic experiments cannot be conducted at general wind tunnels due to the high background noise

in the test section. A good acoustic facility must ensure a low background noise level to meet the essential requirement for aeroacoustic measurements, i.e., the background noise should be at least $10 \mathrm{~dB}$ lower than the noise radiated from models over a wide frequency range. The objective of this work is thus to study the predicted relative performance possibilities of trailing edge serration designs through numerical optimization, and design, manufacture, and test the optimized serration TE to confirm optimization results.

\subsection{Wind tunnel characterization}

The experiments were conducted in the medium-speed, subsonic, closed-loop wind tunnel at Carleton University (as shown in Figure 3.1). The airflow is powered by a $37.3 \mathrm{~kW}$ (50 HP) variable-speed DC motor driving a $1.2 \mathrm{~m}$ axial propeller at speeds as high as 900 RPM. A variable frequency drive (VFD) modulates the rotational frequency of the fan at a resolution of $1.0 \mathrm{~Hz}$, and a pitot-static probe, located just downstream of the inlet, was used to calibrate the linear relationship between motor frequency and wind tunnel velocity. From the calibration, it was determined that the VFD could control the flow speed in increments of $0.9 \mathrm{~m} / \mathrm{s}$, up to a maximum speed of approximately $40 \mathrm{~m} / \mathrm{s}$. Nowadays, due to safety and component long-range issues, this velocity is limited, in this study, to $35 \mathrm{~m} / \mathrm{s}$. A series of turbulence grids precede a 9:1 contraction, which reduces the turbulence intensity levels in the center of the test section to less than $0.27 \%$, as measured for speeds up to $15 \mathrm{~m} / \mathrm{s}$. The tunnel has a removable, rectangular test section measuring $1.83 \mathrm{~m}$ in length and $0.78 \mathrm{~m} \times 0.51 \mathrm{~m}$ at the inlet in width and height, respectively. 

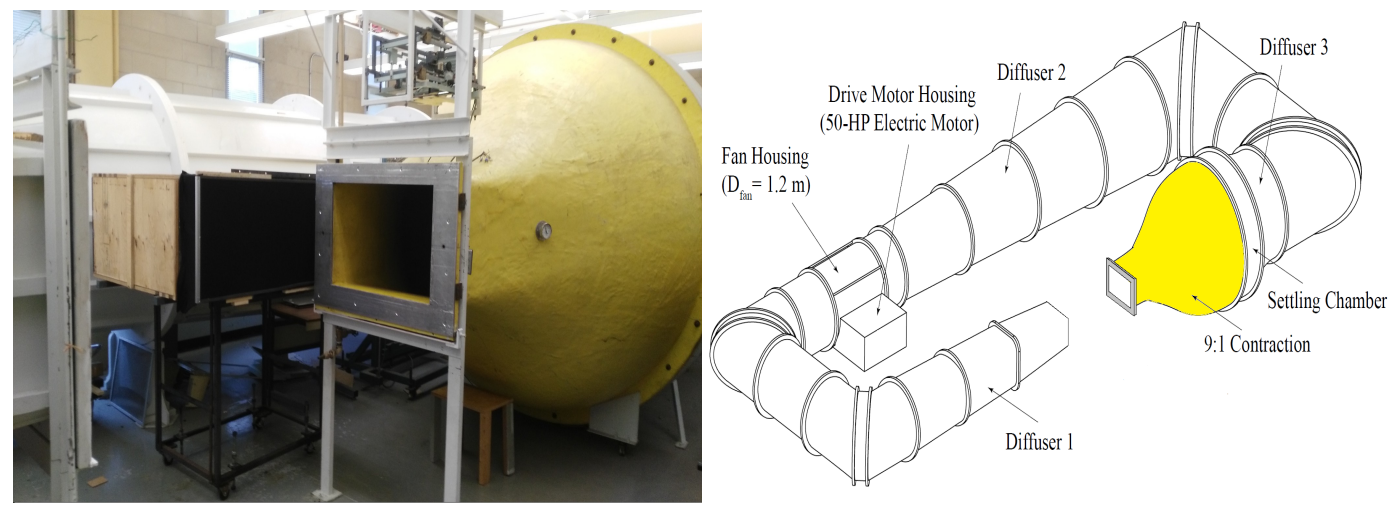

Figure 3.1: Wind tunnel at Carleton University.

\subsection{New aeroacoustic test section}

A new test section (shown in Figures 3.2 and 3.3 ) along with the surrounding anechoic chambers was completed to be used for aeroacoustic testing. This test section is a $0.78 \mathrm{~m}$ $\times 0.51 \mathrm{~m}$ rectangular section, $1.83 \mathrm{~m}$ long. The upper and lower walls of the test section are each composed of two aluminum sheet panels and contain hardware (circle aluminum material) for the vertical mounting of a two-dimensional airfoil (shown in Figure 3.3) midway between the acoustic windows (i.e. test section side walls), and $0.45 \mathrm{~m}$ from the upstream end of the test section. The two sides of the walls of the test section are made of stretched, thin-weave cloth covering a streamwise length of $1.83 \mathrm{~m}$, which provides a smooth flow surface, similar to that of a hard-walled test section, and also a significant reduction in the lift interference effects when compared to that of an open-jet test section. The cloth window allows sound to pass through the walls into the anechoic chambers with very little attenuation. 

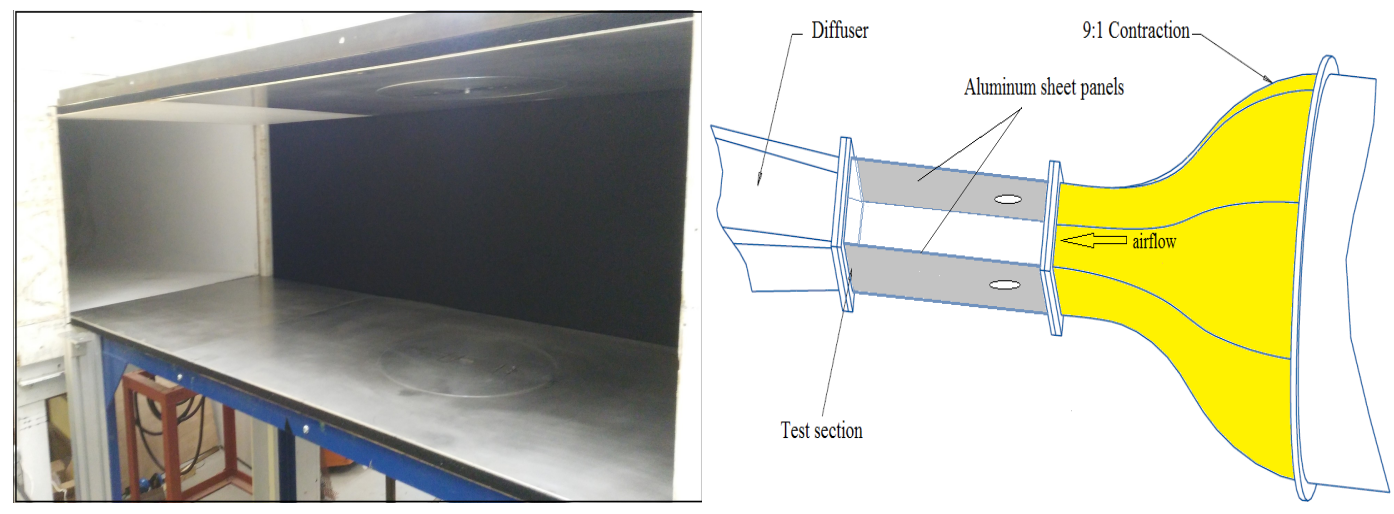

Figure 3.2: Aeroacoustic test section.
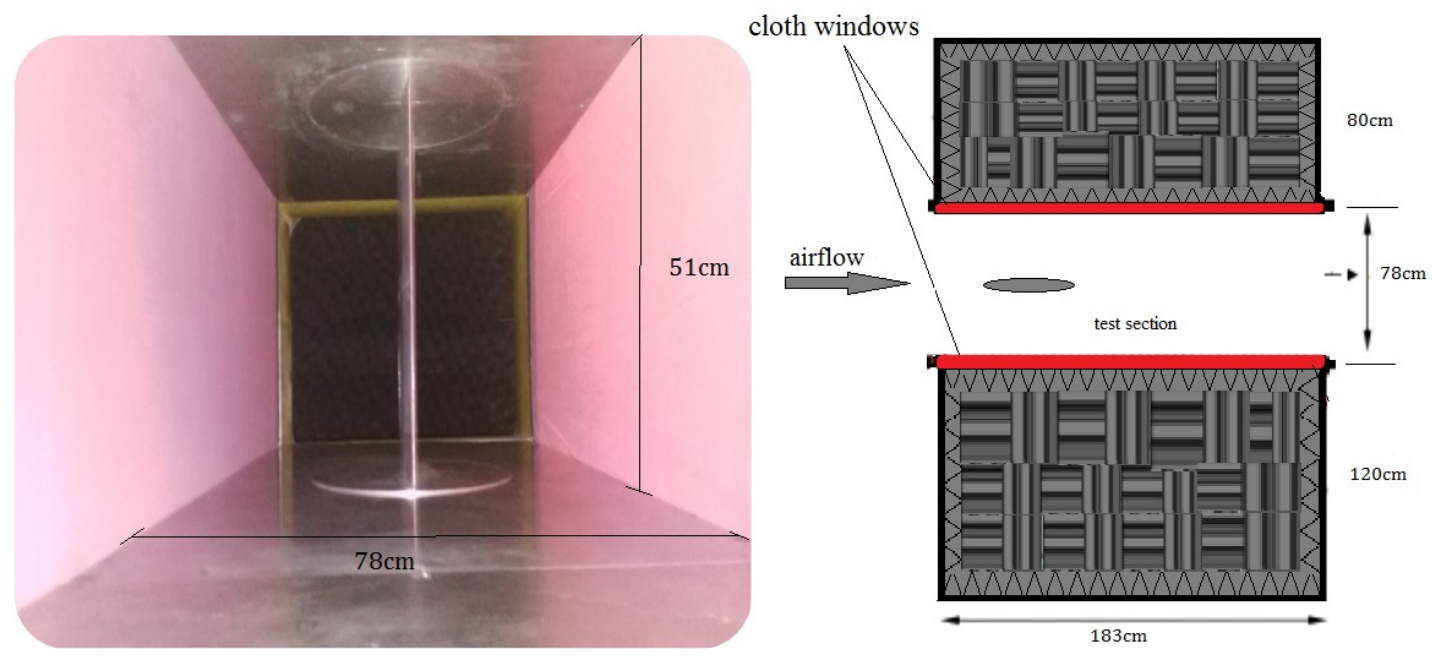

Figure 3.3: Right: a cross-section through the aeroacoustics test section and anechoic as seen from above. Left: photograph was taken from downstream showing the test section interior.

\subsection{Anechoic system}

\subsubsection{Design considerations}

The design of an anechoic system in the wind tunnel aims to achieve low noise radiation and low residual turbulence in the freestream. Besides acoustic and aerodynamic 
requirements to design anechoic system, there are also budgetary limits, as well as limits for available facility space that must be considered. The acoustical performance target is decided upon by the background noise to be at least $10 \mathrm{~dB}$ lower than the airfoil/flat-plate trailing edge self noise at a freestream velocity. The overall layout of the close wind tunnel with respect to the anechoic chamber, as well as some of the design details for each wind tunnel components, will be presented in the next sections.

\subsubsection{Physical layout}

The wind tunnel has an anechoic system that consists, primarily, of an aeroacoustic test section and two anechoic chambers (shown in Figure 3.3). The two anechoic chambers are positioned on either side of the aeroacoustic test section to capture the sound emitted through the acoustic windows and reduce sound reflections inside the section. The chambers are joined together with bolts and clamps to maintain a pressure seal. Both chambers have the same streamwise length of $1.83 \mathrm{~m}$ and different depths of $0.8 \mathrm{~m}$ right-side, and $1.2 \mathrm{~m}$ left-side. The chambers are lined with $0.015 \mathrm{~m}$ carpet bed and, $0.05 \mathrm{~m}$ acoustic wedged foam designed to reduce acoustic reflections.

Each chamber is sealed to the test section so that there is no airflow through either acoustic window. The regions around each of the acoustic windows are covered with a carpet- bed and acoustic foam transitions to cover up all of the hard surfaces within the chamber. The chambers are equipped with a door for access to the inside of the chamber, and for installation of data acquisition equipment. The entire system is removable so that the wind tunnel can be switched from a hard-walled configuration to an anechoic, and back again. 


\subsubsection{Acoustic transparency of the cloth windows}

As with the characterization of the anechoic chambers, the acoustic transparency of the

cloth windows used for the current study is discussed in detail in Remillieux et al. [68. To investigate the acoustic transparency of the cloth sheet windows, the loudspeaker as a white noise source placed perpendicular to the window in the suction side of the anechoic chamber at a distance of $0.05 \mathrm{~m}$ from the window and a single calibrated microphone (Bruel \& Kjaer (B\&K) 4944-A, $\frac{1}{4}$ inch) was used on the other side of the window to record the sound pressure of the source as shown in Figure 3.4 .

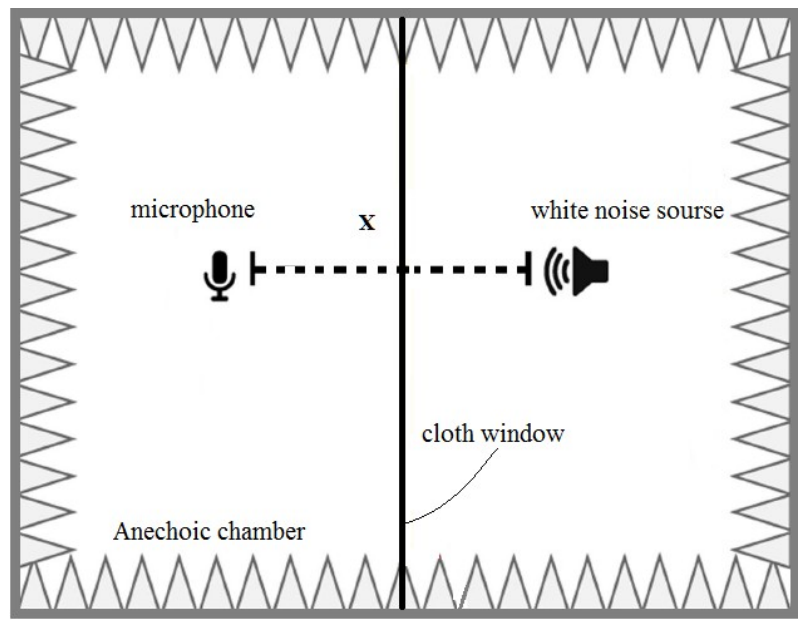

Figure 3.4: Acoustic transparency of the cloth window.

The sound levels, white noise, generated by the source were measured and were compared to the measured levels that the microphone would have been exposed to in the absence of the window. Sound pressure level $(S P L)$ was used to compare the noise levels measured in the anechoic enclosure to those measured in the presence of the cloth window. The measurement is presented in Figure 3.5 . 


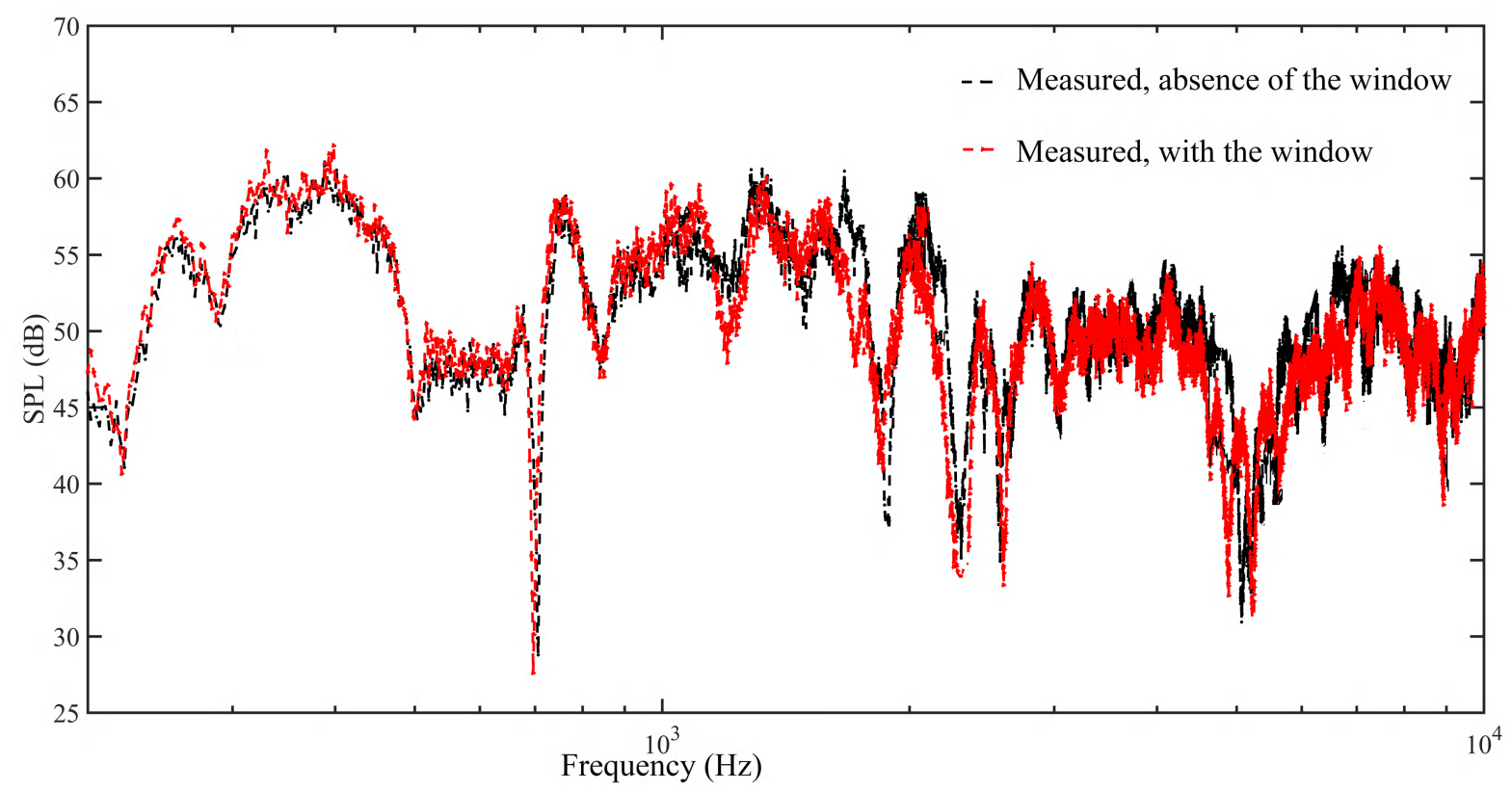

Figure 3.5: Attenuation of sound passing through the acoustic cloth window as a function of frequency.

Figure 3.5 shows that there is minimal loss through the window $(\sim<3 d B)$ for frequencies less than $10 \mathrm{kHz}$. For all measurements presented in this paper, the frequency range of interest is significantly less than $10 \mathrm{kHz}$ and thus the acoustic loss through the sheet windows can be neglected.

\subsection{Airfoil model}

For some of the tests, a NACA-0012 airfoil model was used as a benchmark test. The chord of the airfoil is $0.3 \mathrm{~m}$, and the span is $0.51 \mathrm{~m}$. The $2 \mathrm{D}$ airfoil is manufactured as two halves, each one composed of three pieces with eight screws (see Figure 3.6). Eight holes were drilled on each side of the chord length of the airfoil, so this can be fixed on the circle rotating mechanism. The trailing edge is $0.08 \mathrm{~m}$ wide. The NACA-0012 airfoil wing is mounted vertically in the test section with its leading-edge (at zero angles of attack) 
$0.45 \mathrm{~m}$ downstream of the test section entrance. Angles relative to zero were set by using a calliper and scribe lines on the steel floor plate immediately beneath the model.
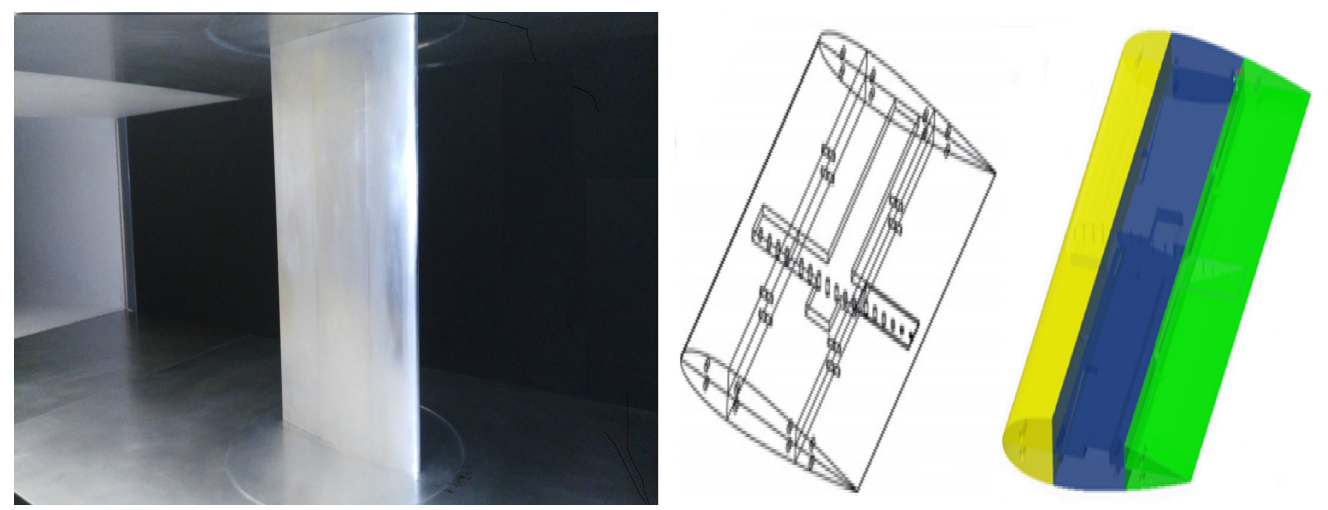

Figure 3.6: 2-D airfoil schematic.

\subsection{Noise source model}

In this section, the mathematical models for the prediction of noise used in this paper are presented. The underlying derivation for the noise models was originally given by Howe for sinusoidal serrations [60], and then soon after for sawtooth serrations [3]. In recent years, the model for slitted trailing edge serrations was presented by Gruber et al [37. The modelling is based on Howe's derivation of the problem [3] in which pressure fluctuations in the TBL are scattered into radiated noise by the discontinuity in the acoustic impedance that occurs at the trailing edge and includes the following assumptions:

- The flow is of low Mach number.

- The model has infinite span.

- Frozen turbulence convected past the trailing edge of a semi-infinite flat plate. 
- The Kutta condition is satisfied.

- No other extraneous noise sources are present.

Figure 3.7 shows a sketch of the the four geometries considered in this study. The expressions defining the sawtooth, slitted and sinusoidal geometry are given in equations 3.1 . 3.2 , and 3.3 respectively.

Sawtooth edge :

$y_{1}=\Gamma\left(y_{3}\right)=\left\{\begin{array}{rr}(4 h / \lambda)\left(y_{3}-n \lambda\right), & n \lambda<y_{3}<\left(n+\frac{1}{2}\right) \lambda \\ -(4 h / \lambda)\left(y_{3}-n \lambda\right), & \left(n-\frac{1}{2}\right) \lambda<y_{3}<n \lambda\end{array}, n=0, \pm 1, \pm 2, \pm 3, \ldots\right.$

Slitted edge :

$y_{1}=\Gamma\left(y_{3}\right)=\left\{\begin{array}{cc}-h, & n\left(\lambda_{1}+\lambda_{2}\right)<y_{3}<(n+1) \lambda_{1}+n \lambda_{2} \\ h, & (n+1) \lambda_{1}+n \lambda_{2}<y_{3}<(n+1)\left(\lambda_{1}+\lambda_{2}\right)\end{array}, n=0, \pm 1, \pm 2, \pm 3, \ldots\right.$

Sinusoidal edge :

$$
y_{1}=\Gamma\left(y_{3}\right)=h \cos \left(2 \pi \frac{y_{3}}{\lambda}\right)
$$

The scattered pressure produced at the trailing edge is defined in terms of the Green's function and the turbulence blocked pressure, $P_{b}$, as follows $[3]$ :

$$
P_{s}(x ; \omega)=\frac{i}{2} \int_{-\infty}^{\infty} d y_{3} \int_{-\infty}^{0} d z_{1} \int_{-\infty}^{\infty} \gamma(K)\left[G\left(x, y_{1}, y_{3} ; \omega\right)\right] P_{b}(K ; \omega) e^{i\left(K_{1} y_{1}+K_{3} y_{3}\right)} d^{2} K
$$




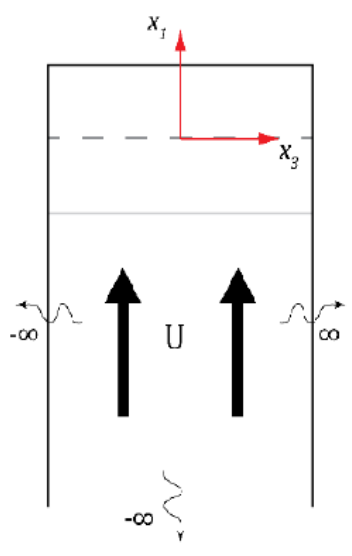

[a]

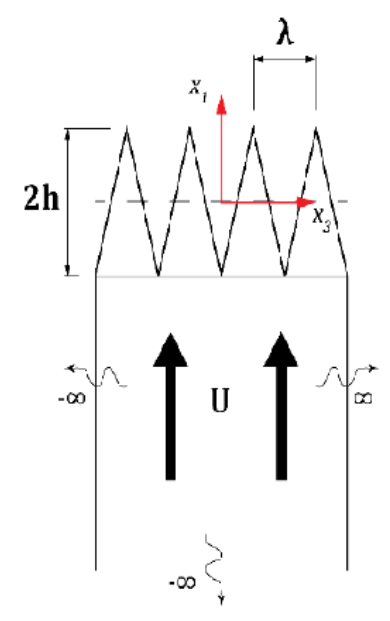

[b]

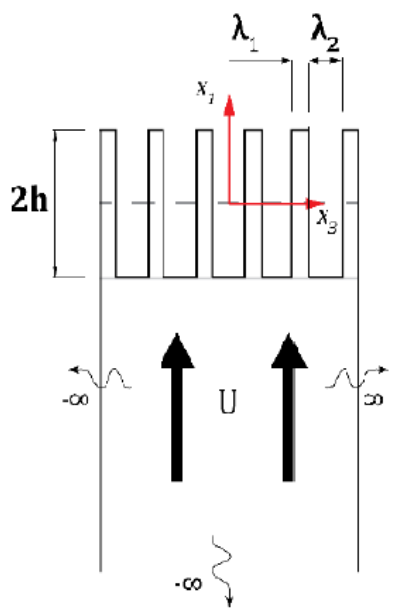

[c]

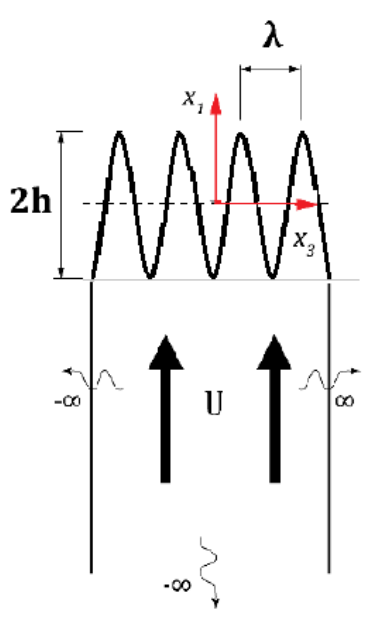

[d]

Figure 3.7: 2-D schematic drawings of flat plate [a] Straight, [b] Swatooth, [c] Slitted, and, [d] Sinusoidal TE extensions.

where $\mathbf{K}=\left(K_{1}, 0, K_{3}\right)$ is the boundary layer turbulent wavenumber vector, and $\gamma(K)=\sqrt{\left(\kappa^{2}-|\mathbf{K}|^{2}\right)}$, for $\kappa>|\mathbf{K}|$ and $\gamma(K)=i \sqrt{\left(|\mathbf{K}|^{2}-\kappa^{2}\right)}$, for $\kappa<|\mathbf{K}|$.

The Green's function is given by Howe in [60] and is based on applying the slenderwing approximation to the Green's function for straight trailing edges. $P_{b}$ is obtained from an empirical model for the turbulent wall pressure wave-number-frequency spectrum developed by Chase 69]:

$$
P_{b}(K, \omega)=\frac{C_{m} \rho^{2} u_{*}^{3} K_{1}^{2} \delta^{5}}{\left[\left(K_{1}-\frac{\omega}{U_{c}}\right)^{2}\left(\frac{\delta U_{c}}{3 u_{*}}\right)^{2}+(|K| \delta)^{2}+\varepsilon^{2}\right]^{5 / 2}}
$$

in which the friction velocity, $u_{*}=0.03 U$, the convection velocity, $U_{c}=0.7 U$, and the empirical constants, $C_{m}=0.1553$ and $\varepsilon=1.33$.

The final, non-dimensional, forms of the trailing edge noise spectrum for the straight, sawtooth, slitted and sinusoidal trailing edge geometries are the following : 


$$
\begin{aligned}
& \Psi_{0}(\omega)=\frac{\left(\frac{\omega \delta}{U_{c}}\right)^{2}}{\left[\frac{\omega \delta}{U_{c}}+\varepsilon^{2}\right]^{2}} \\
& \Psi_{\text {saw }}(\omega)=8\left(\frac{h}{\delta}\right)^{2}\left(\frac{\omega h}{U_{c}}\right) \sum_{n=-\infty}^{\infty} \frac{\left[1-\cos \left(\frac{2 \omega h}{U_{c}}\right) / \cos (n \pi)\right]\left[\left(\frac{\omega h}{U_{c}}\right)^{2}+\left(2 n \pi \frac{h}{\lambda}\right)^{2}\right]}{\left[(n \pi)^{2}-\left(\frac{2 \omega h}{U_{c}}\right)^{2}\right]^{2}\left[\left(\frac{\omega h}{U_{c}}\right)^{2}+\left(2 n \pi \frac{h}{\lambda}\right)^{2}+\left(\frac{\varepsilon h}{\delta}\right)^{2}\right]^{2}} \\
& \Psi_{\text {slit }}(\omega)=\sum_{n=-\infty}^{\infty} \Theta \Theta^{*} \frac{\left[\left(\frac{\omega \delta}{U_{c}}\right)^{2}+\left(2 n \pi \frac{\delta}{\left(\lambda_{1}+\lambda_{2}\right)}\right)^{2}\right]}{\left[\left(\frac{\omega \delta}{U_{c}}\right)^{2}+\left(2 n \pi \frac{\delta}{\left(\lambda_{1}+\lambda_{2}\right)}\right)^{2}+\left(\varepsilon^{2}\right)^{2}\right]} \\
& \Theta\left(K, \lambda_{1}, \lambda_{2}, h\right)=n^{-1}\left[\left(e^{\frac{2 i n \pi \lambda_{1}}{\left(\lambda_{1}+\lambda_{2}\right)}}-1\right) e^{i K_{1} h}+\left(1-e^{\frac{-2 i n \pi \lambda_{1}}{\left(\lambda_{1}+\lambda_{2}\right)}}\right) e^{-i K_{1} h}\right] \\
& \Psi_{s i n}(\omega)=\left(\frac{\omega h}{U}\right) \sum_{n=-\infty}^{\infty} J_{n}^{2}\left(\frac{\omega h}{U}\right) \frac{\left(\frac{\omega \delta}{U}\right)^{2}+\left(2 n \pi \frac{\delta}{\lambda}\right)^{2}}{\left[\left(\frac{\omega \delta}{U}\right)^{2}+\left(2 n \pi \frac{\delta}{\lambda}\right)^{2}+\varepsilon^{2}\right]^{2}}
\end{aligned}
$$

The boundary layer thickness, $\delta$, at the airfoil trailing is an important parameter in determining the noise performance of trailing edge serrations. The TBL thickness used in the noise prediction is calculated as following [70,71:

$$
\delta=\frac{0.37 c\left[1+\left(\frac{R e_{c}}{6.9 \times 10^{7}}\right)^{2}\right]^{1 / 10}}{R e_{c}^{\frac{1}{5}}}
$$

The non-dimensional overall sound pressure level $\left(O A S P L_{n o r m}\right)$ describes the total amount of noise produced across the desired frequency range. The $O A S P L_{n o r m}$ is used both an objective function and for the comparison of various trailing edge designs to a straight trailing edge. The $O A S P L_{\text {norm }}$ is calculated as follows:

$$
O A S P L_{n o r m}=10 \log _{10}\left(\int_{\omega_{\min }}^{\omega_{\max }} \Psi(\omega) d \omega\right)
$$


where $\omega_{\min }$ and $\omega_{\max }$ are the lower and upper bounds on the frequency range of interest respectively and, $\Psi(\omega)$ can be obtained from any of equations 3.6 to 3.10 according to the geometry of interest.

\subsection{Numerical optimization methods}

This study examines three different optimization problems, which are given below in proper form in equations. 3.13 to 3.15. These equations correspond to the single-size optimization of sawtooth (see Figure 3.7b), slitted (see Figure 3.7p) and sinusoidal (see Figure 3.7d) TE geometries, respectively. In each case, the optimum design is the singlesize of serration that produces the least amount of total noise overall frequencies between $0.1 \mathrm{~Hz}$ and $10 \mathrm{kHz}$.

$$
\begin{aligned}
& \left\{\begin{array}{cc}
\text { minimize } & Z=O A S P L_{\text {norm saw }}(h, \lambda) \\
h, \lambda & \\
\text { subject to } & 0 \leq h \leq h_{\max } \\
& \lambda_{\min } \leq \lambda \leq \lambda_{\max }
\end{array}\right. \\
& \begin{cases}\text { minimize } & Z=O A S P L_{\text {norm slit }}\left(h, \lambda_{1}, \lambda_{2}\right) \\
h, \lambda_{1}, \lambda_{2} & \\
\text { subject to } & 0 \leq h \leq h_{\max } \\
& \left(\lambda_{1}\right)_{\min } \leq \lambda_{1} \leq\left(\lambda_{1}\right)_{\max } \\
& (\lambda 2)_{\min } \leq \lambda 2 \leq(\lambda 2)_{\max }\end{cases}
\end{aligned}
$$




$$
\begin{cases}\text { minimize } & Z=O A S P L_{\text {norm } \sin }(h, \lambda) \\ h, \lambda & \\ \text { subject to } & 0 \leq h \leq h_{\max } \\ & \lambda_{\min } \leq \lambda \leq \lambda_{\max }\end{cases}
$$

where $Z$ is the objective function value; $h_{\max }$ is the largest feasible serration amplitude corresponding to the sawtooth, slitted and sinusoidal geometries accordingly; $\left(\lambda_{\min }\right)$ and $\left(\lambda_{\max }\right)$ are the smallest and largest feasible sawtooth and sinusoidal widths, respectively; $\left(\lambda_{1}\right)_{\min }$ and $\left(\lambda_{1}\right)_{\max }$ are the smallest and largest feasible slit widths, respectively; and $\left(\lambda_{2}\right)_{\min }$ and $\left(\lambda_{2}\right)_{\max }$ are the smallest and largest feasible gap widths, respectively. A summary of the optimization studies conducted for this work is presented in Table 3.1 . 
Table 3.1: Optimization trials and associated design variable (DV) bounds for $(U=$ $24 \mathrm{~m} / \mathrm{s}$ )

\begin{tabular}{cccccccc}
\hline \multirow{2}{*}{ Study No } & Geometry & \multicolumn{3}{c}{ Lower DV Bound (mm) } & \multicolumn{3}{c}{ Upper DV Bound (mm) } \\
\cline { 3 - 8 } & & $\lambda_{1 \text { min }}$ & $\lambda_{2 \min }$ & $h_{\min }$ & $\lambda_{1 \max }$ & $\lambda_{2 \max }$ & $h_{\max }$ \\
\hline SS-Saw-1 & Sawtooth & 10 & - & 0 & 30 & - & 35 \\
SS-Saw-2 & Sawtooth & 15 & - & 0 & 35 & - & 40 \\
SS-Saw-3 & Sawtooth & 20 & - & 0 & 40 & - & 30 \\
SS-Saw-4 & Sawtooth & 25 & - & 0 & 35 & - & 37.5 \\
\hline SS-Slit-1 & Slitted & 10 & 10 & 0 & 40 & 30 & 35 \\
SS-Slit-2 & Slitted & 15 & 20 & 0 & 35 & 40 & 40 \\
SS-Slit-3 & Slitted & 10 & 25 & 0 & 30 & 35 & 37.5 \\
SS-Slit-4 & Slitted & 20 & 20 & 0 & 30 & 40 & 40 \\
\hline SS-Sinu-1 & Sinusoidal & 15 & - & 0 & 40 & - & 35 \\
SS-Sinu-2 & Sinusoidal & 10 & - & 0 & 35 & - & 30 \\
SS-Sinu-3 & Sinusoidal & 20 & - & 0 & 45 & - & 40 \\
SS-Sinu-4 & Sinusoidal & 25 & - & 0 & 30 & - & 37.5 \\
\hline
\end{tabular}

\subsection{Results and discussion}

\subsubsection{Background noise levels}

The background noise of the wind tunnel is measured in the anechoic chamber with a single calibrated B\&K microphone. Figure 3.8 shows empty test-section background sound pressure levels $(S P L)$ in the starboard-side anechoic chamber as a function of flow speed, $0 \mathrm{~m} / \mathrm{s}$ to $24 \mathrm{~m} / \mathrm{s}$. These measurements were made $1.4 \mathrm{~m}$ from the test-section center. 


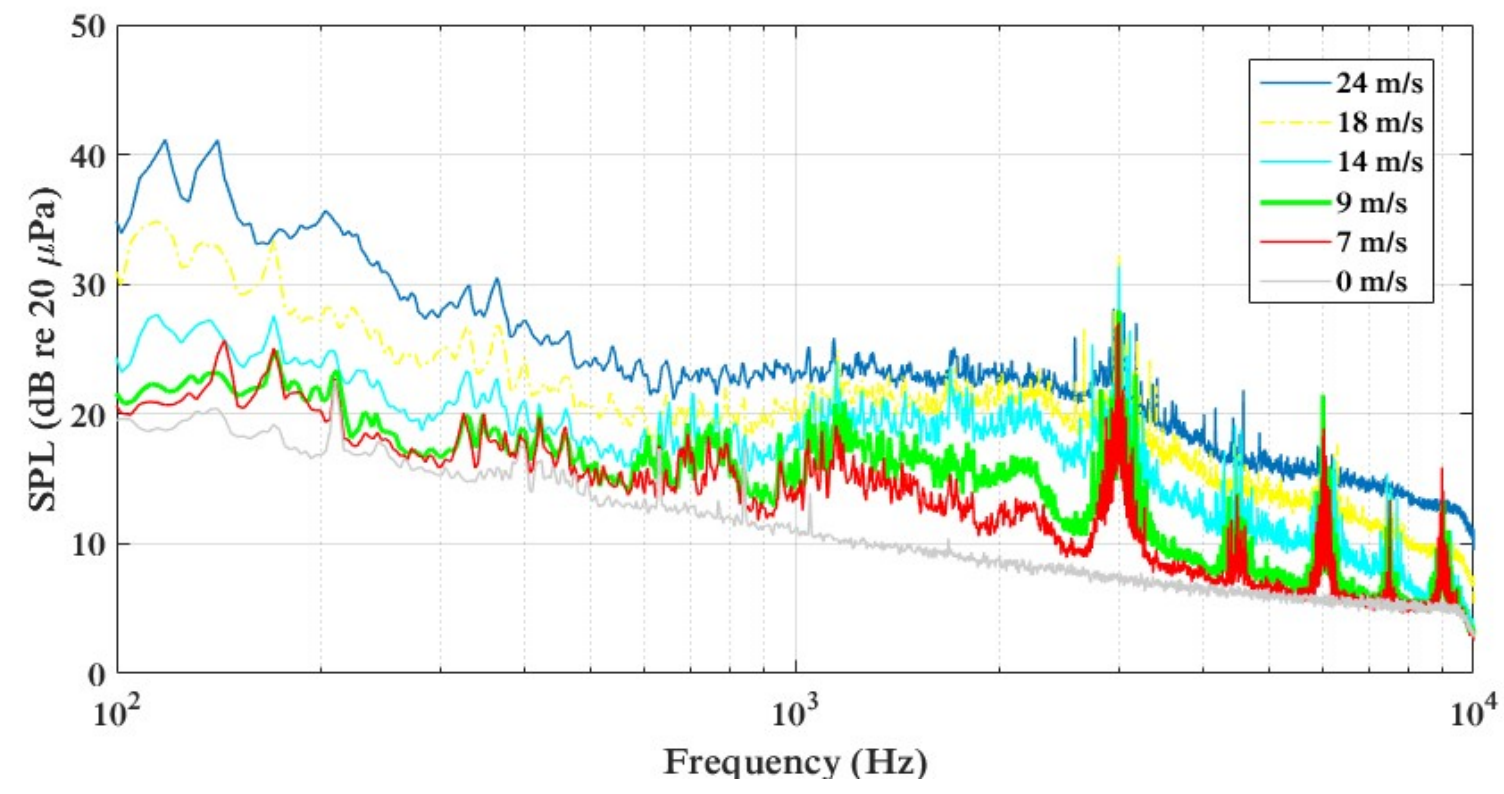

Figure 3.8: SPL in the starboard-side anechoic chamber $(1.4 \mathrm{~m}$ from the test-section center) as a function of flow speed in the empty test section.

The highest spectral level can be seen at low frequencies $(100-500 \mathrm{~Hz})$. Background noise levels at frequencies less than $500 \mathrm{~Hz}$ are mostly tones generated by the wind tunnel fan and levels at frequencies greater than $500 \mathrm{~Hz}$ are primarily broadband and believed to be due to a combination of noise sources including the fan, turning vanes, and scrubbing noise from flow surfaces in and around the test section. The peaks showed in $3 \mathrm{kHz}$, $4.5 \mathrm{kHz}$ and $6 \mathrm{kHz}$ are mostly associated with motor tones. The Overall Sound Pressure Level, $O A S P L$, is obtained by integrating the noise spectrum from $0.1 \mathrm{kHz}$ to $10 \mathrm{kHz}$. Figure 3.9 shows the dependence of $O A S P L$ on free-stream velocity; the $5^{\text {th }}$ power law is satisfied, which is similar to other closed-circuit type wind tunnels. 


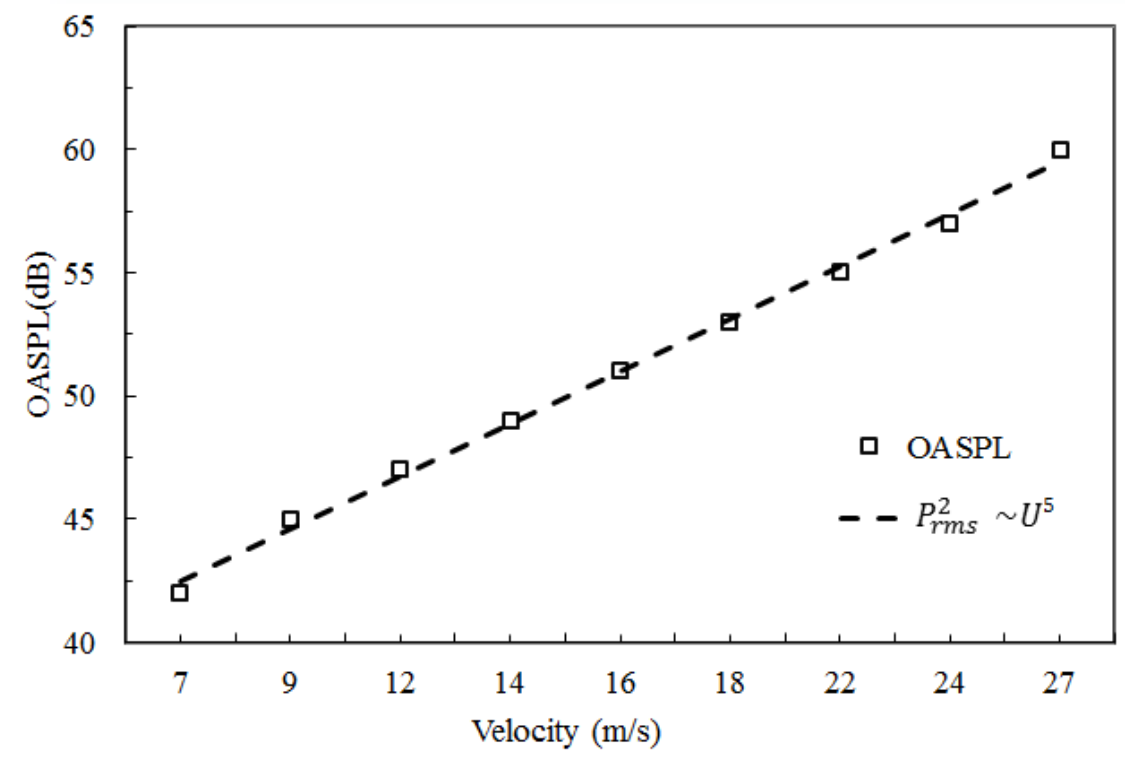

Figure 3.9: The overall sound pressure level of background noise as a function of freestream velocity.

To estimate the acoustic performance, the A-weighted overall sound pressure level, $O A S P L$, is compared to other acoustic facilities around the world. Since the nozzle dimensions and microphone positions are different from each other, the measured results must be transformed before comparing with each other [3]:

$$
O A S P L_{\text {corrected }}=O A S P L_{\text {measured }}-10 \log _{10}\left(\frac{S}{R^{2}}\right)
$$

where $\mathrm{R}$ and $\mathrm{S}$ are the distance from the microphone to the wind tunnel center-line and nozzle exit area, respectively.

The background noise of Carleton University wind tunnel is scaled using equation 3.16 and the results are shown in Figure 3.10. The background noise of other acoustic facilities with data obtained from the literature 72,75 is also plotted in Figure 3.10 for comparison. The results indicate that the background noise of the Carleton University wind tunnel is comparable with other aeroacoustic wind tunnels. 


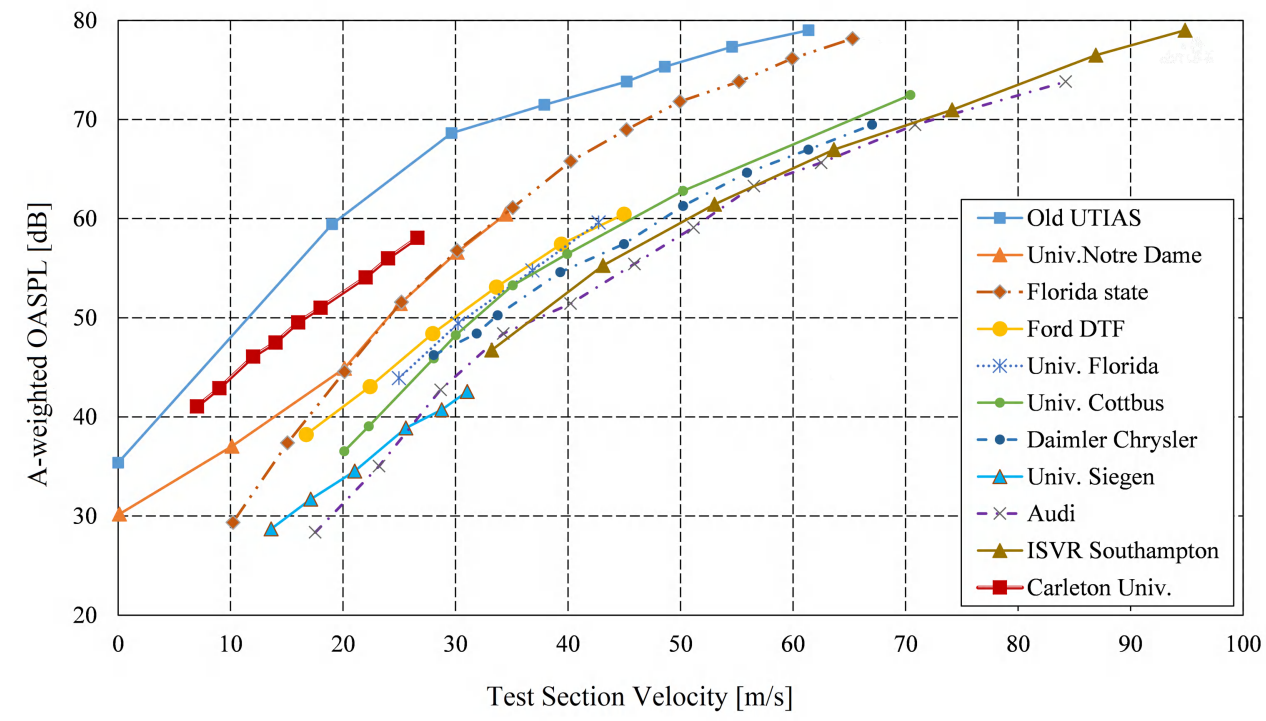

Figure 3.10: Comparison of A-weight sound pressure level of Carleton University wind tunnel to other acoustic facilities worldwide.

\subsubsection{Far-field trailing edge noise measurements}

\subsubsection{Measurements of the airfoil TE noise}

NACA-0012 airfoil with straight and sawtooth trailing edge (see Figure 3.11) was submerged within the potential core of the jet to assess the trailing edge self-noise in relation to the wind tunnel background noise. The airfoil was held at zero angles of attack by side plates extended from the nozzle sidewalls. The radiated noise was measured at 1.4 $\mathrm{m}$ from the center of the trailing edge in the starboard-side, which corresponds to a $90^{0}$ of polar angle $\theta$.

At first, the background noise of the wind tunnel was measured under a free-stream velocity of $14 \mathrm{~m} / \mathrm{s}$ and $24 \mathrm{~m} / \mathrm{s}$. The airfoil with straight $\mathrm{TE}$ as a reference and the same airfoil with sawtooth TE were then attached to the sidewalls, and the same free-stream velocities were repeated. The result of the TE self-noise spectra of these cases is plotted in Figure 3.11. The Figure shows that the serration geometry is effective in reducing the 
trailing edge noise component. The TE self noise measurement is seen to be from $\sim(5$ to 15$) \mathrm{dB}$ above the background wind tunnel noise within the frequency range from 0.1 $\mathrm{kHz}$ to $\sim 3 \mathrm{kHz}$, which guarantees the validity of the results.

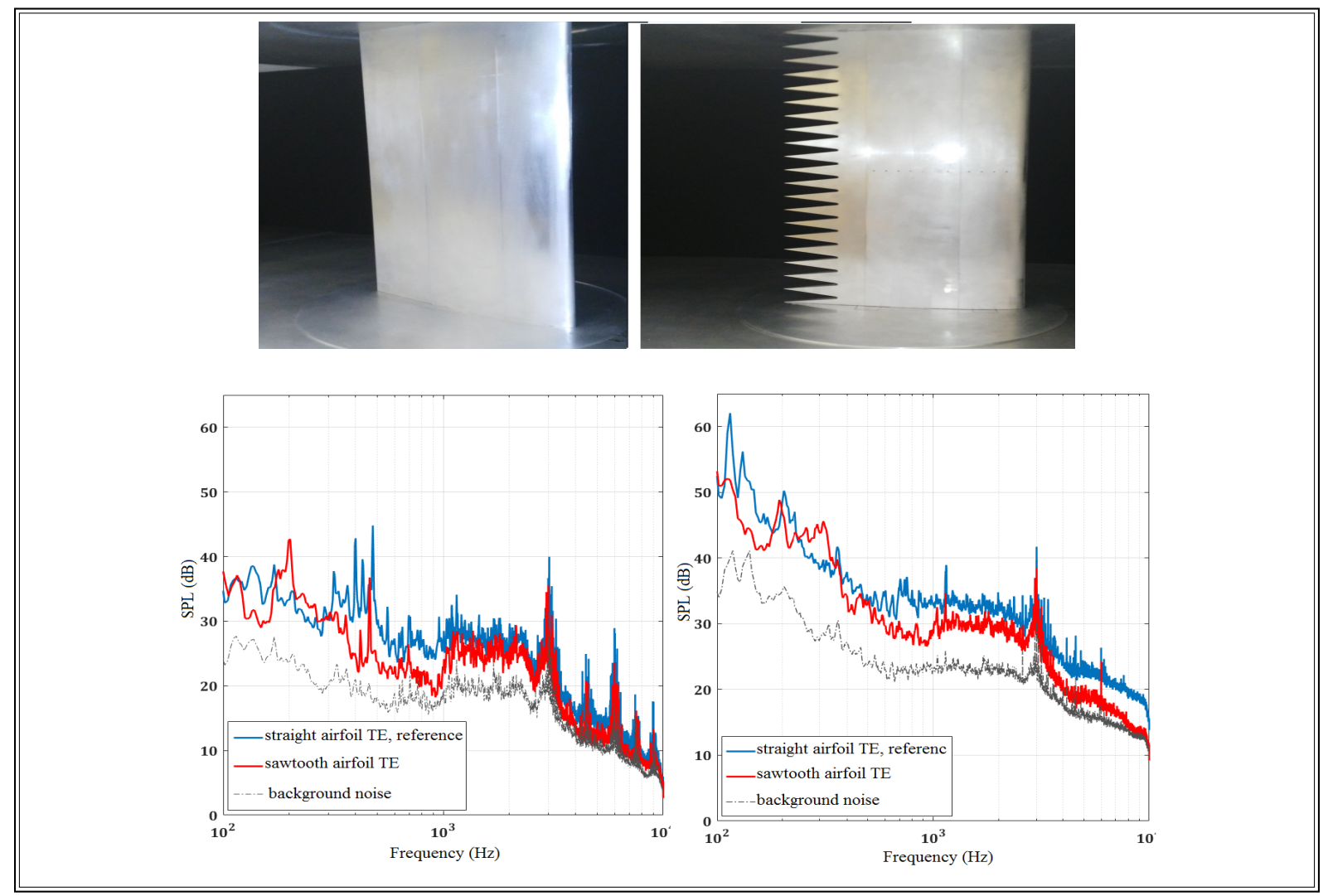

Figure 3.11: Measured SPL spectra for NACA-0012 airfoil and the same airfoil with sawtooth TE at free-stream velocities of (Left) $14 \mathrm{~m} / \mathrm{s}$ and (Right) $24 \mathrm{~m} / \mathrm{s}$ at Carleton University anechoic wind tunnel. The background noise spectra are also shown in this figure for comparison.

\subsubsection{Measurements of the flat-plate TE noise}

A $3 \mathrm{~mm}$ thickness flat-plate, $0.30 \mathrm{~m}$ in chord and $0.51 \mathrm{~m}$ in span, was submerged within the potential core of the jet to assess the trailing edge self-noise in relation to the wind tunnel background noise. The flat-plate was held at zero angles of attack by side plates extended from the nozzle sidewalls. The radiated noise was measured at $1.4 \mathrm{~m}$ from the 
center of the trailing edge in the starboard-side, which corresponds to a $90^{\circ}$ of polar angle $\theta$.

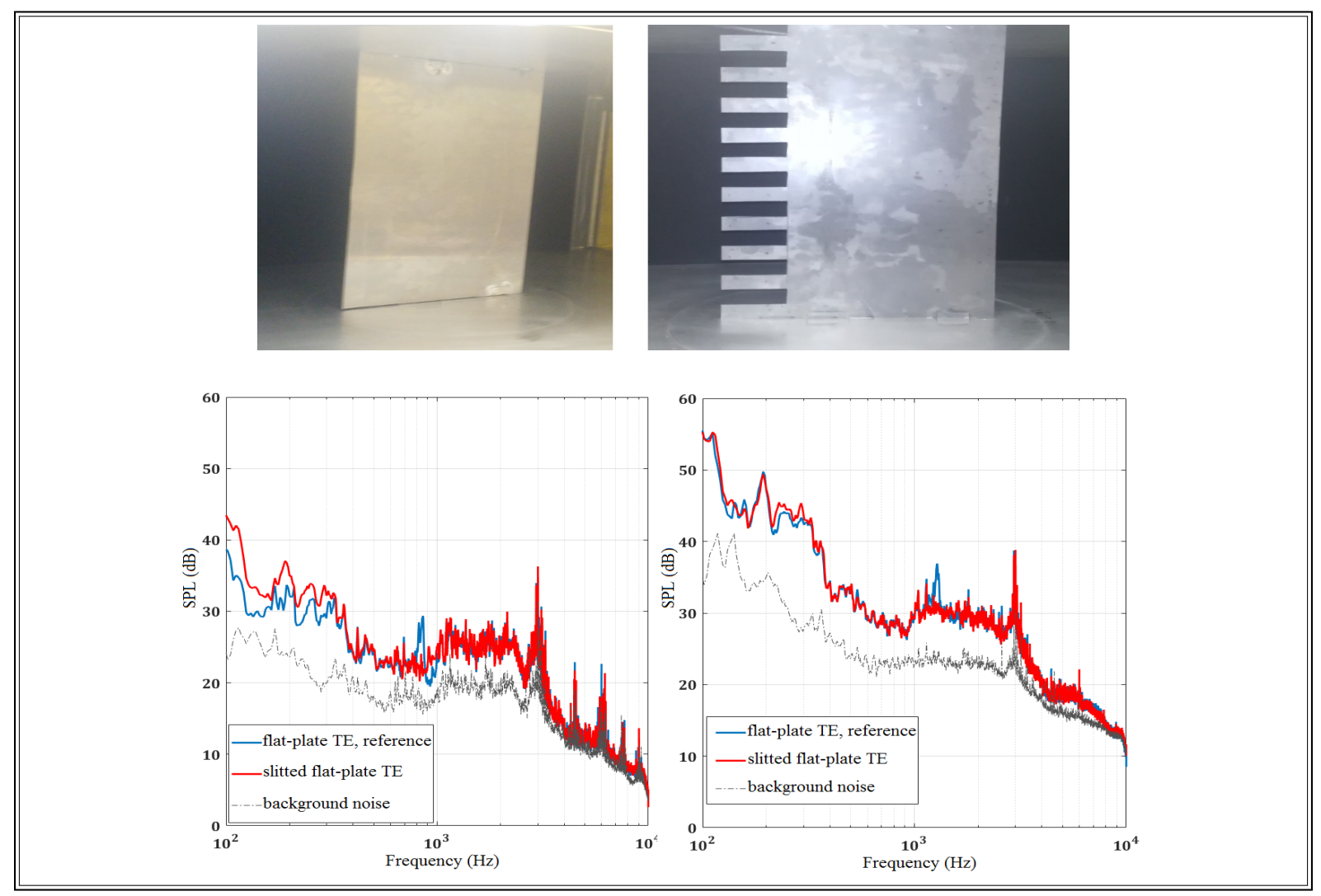

Figure 3.12: Far-field acoustic spectra for the reference flat-plate and the flat-plate with trailing edge serrations, slitted TE, at freestream velocities of (Left) $14 \mathrm{~m} / \mathrm{s}$ and (Right) $24 \mathrm{~m} / \mathrm{s}$. The background noise spectra are also shown in this figure for comparison.

Similarly, to the airfoil, the background noise of the wind tunnel was measured first under a freestream velocity of $14 \mathrm{~m} / \mathrm{s}$ and $24 \mathrm{~m} / \mathrm{s}$. The flat-plate with straight TE as a reference and the same flat-plate with slitted TE were then attached to the sidewalls, and the same freestream velocities were repeated. The result of the TE self-noise spectra of these cases is plotted in Figure 3.12 . The figure indicates that the serration geometry is effective in reducing the trailing edge self-noise component and the TE self-noise measurement is seen to be from $\sim 4$ to $\sim 16 \mathrm{~dB}$ above the background wind tunnel noise, within 
the frequency range from $0.1 \mathrm{kHz}$ to $\sim 3 \mathrm{kHz}$, which guarantees the validity of the results.

\subsubsection{Optimization results}

This section presents the optimization results from the analyses that used the sawtooth, slitted and sinusoidal geometry. The serrations designs are optimized to find the single size of each tooth geometry that produces the least noise over the entire frequency spectrum of interest $(0.1 \mathrm{kHz}$ to $10 \mathrm{kHz})$ for each set of constraints examined. The optimization code developed for this study is based on MATLAB. It uses an interior point algorithm for a nonlinear equation. The equations being analyzed are nonlinear functions. An interior point algorithm is an approach to constrained minimization by solving a sequence of approximate minimization problems. A summary of the optimized designs from each study, and their respective $O A S P L_{\text {norm }}$, is shown in Table 3.2 . 
Table 3.2: Optimized designs from each study and noise produced by each trailing edge.

\begin{tabular}{|c|c|c|c|c|c|c|}
\hline \multirow[t]{2}{*}{ Study No } & \multicolumn{3}{|c|}{$\begin{array}{l}\text { Optimal Geometry } \\
(\mathrm{mm})\end{array}$} & \multirow[t]{2}{*}{$\begin{array}{c}O A S P L_{\text {norm }} \\
{[\mathrm{dB}]}\end{array}$} & \multirow[t]{2}{*}{$\begin{array}{c}\int_{\omega_{1}}^{\omega_{2} \Psi_{i}=} \\
10^{\frac{O A S P_{\text {norm }}}{10}}\end{array}$} & \multirow[t]{2}{*}{$\begin{array}{c}\triangle O A S P L_{\text {norm }} \\
{[\mathrm{dB}]}\end{array}$} \\
\hline & $\lambda_{\text {lopt }}$ & $\lambda_{2 o p t}$ & $h_{o p t}$ & & & \\
\hline Straight TE & - & - & - & 30.1 & 1023.3 & - \\
\hline SS-Saw-1 & 10 & - & 35 & 16.7 & 46.7 & 13.3 \\
\hline SS-Saw-2 & 15 & - & 40 & 18.2 & 66.1 & 11.9 \\
\hline SS-Saw-3 & 20 & - & 30 & 21.5 & 141.2 & 8.6 \\
\hline SS-Saw-4 & 25 & - & 37.5 & 21.6 & 181.9 & 7.5 \\
\hline SS-Slit-1 & 10 & 10 & 6.4 & 27.9 & 616.6 & 2.2 \\
\hline SS-Slit-2 & 15 & 20 & 5.8 & 28.5 & 707.9 & 1.6 \\
\hline SS-Slit-3 & 10 & 25 & 6.0 & 28.5 & 707.9 & 1.6 \\
\hline SS-Slit-4 & 20 & 20 & 5.6 & 28.6 & 724.4 & 1.5 \\
\hline SS-Sinu-1 & 15 & - & 35 & 21.1 & 128.8 & 9 \\
\hline SS-Sinu-2 & 10 & - & 30 & 20.0 & 100 & 10.1 \\
\hline SS-Sinu-3 & 20 & - & 40 & 21.9 & 154.9 & 8.2 \\
\hline SS-Sinu-4 & 25 & - & 37.5 & 23.1 & 204.2 & 7 \\
\hline
\end{tabular}

\subsubsection{Verification of the noise model}

Noise models previously defined in equations 3.6 to 3.10 were coded in Matlab to be used. In order to verify the Matlab code, the function outputs were compared to results published by Howe et al. [3] (for equation 3.7), by Azarpeyvand et al. [4] (for equation 3.8) and by Howe et al. [3] (for equation 3.10). Based on the thorough comparison in this analysis, it was concluded that the Matlab functions used produce identical results to the analytical equivalents, as shown in Figures $3.13,3.14$ and 3.15 . 


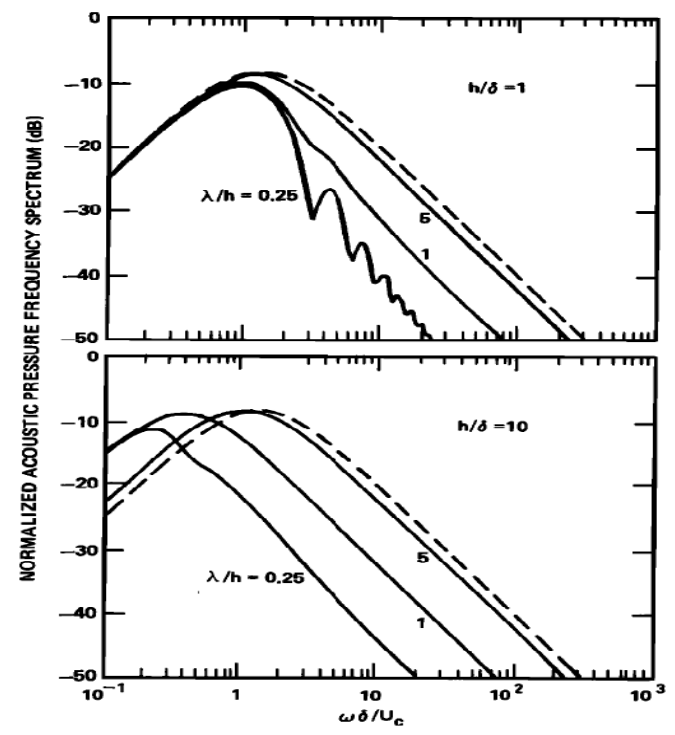

(a)

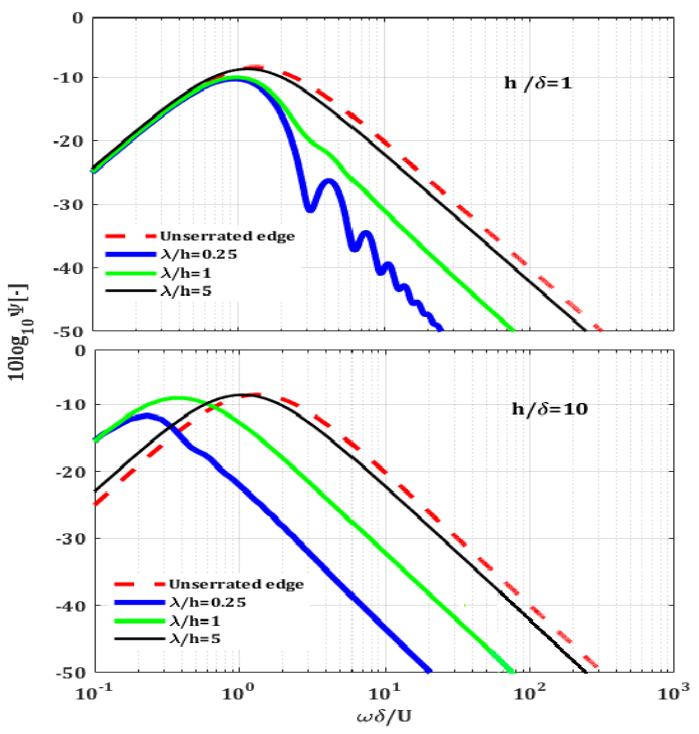

(b)

Figure 3.13: Normalized spectrum of noise produced by a low Mach number flow over a sawtooth TE: (a) from [3] equation (17) ;(b) Matlab code..

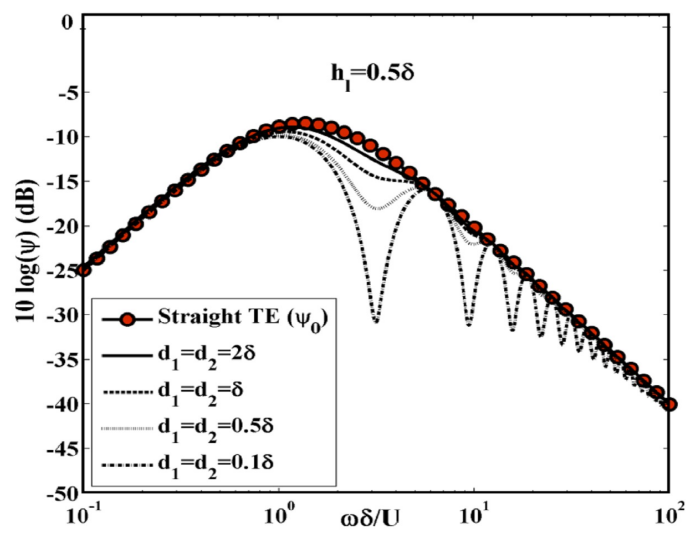

(a)

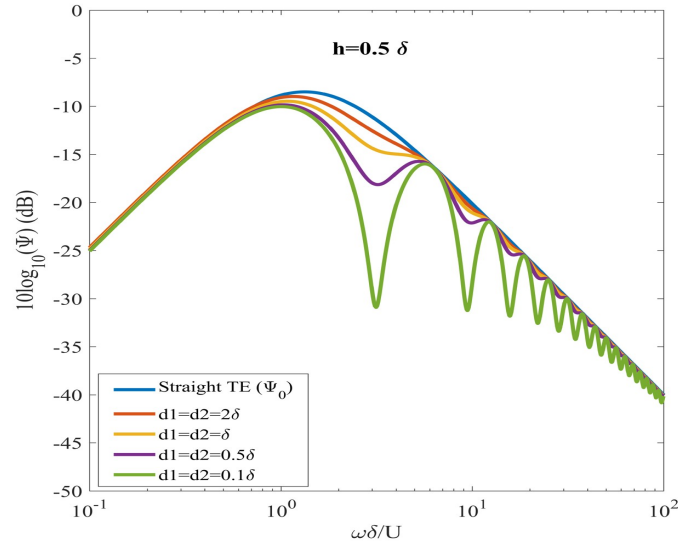

(b)

Figure 3.14: Normalized spectrum of noise produced by a low Mach number flow over a slitted TE: (a) from [4 equation (10); (b) Matlab Code. 


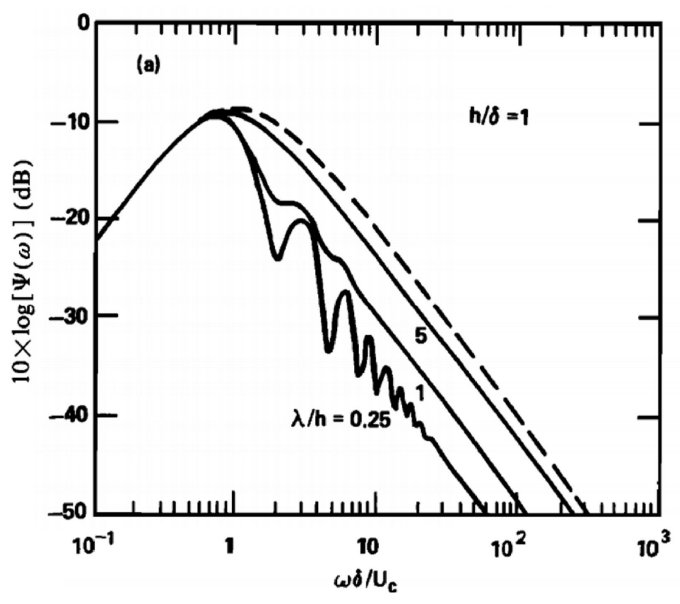

(a)

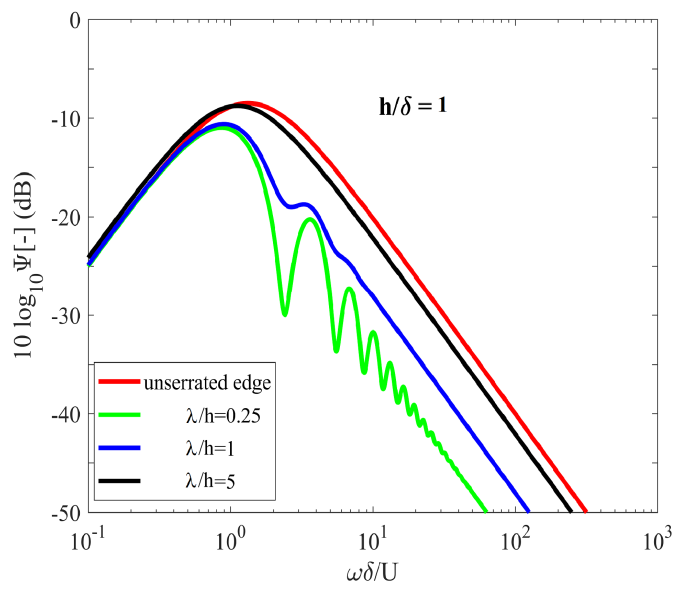

(b)

Figure 3.15: Normalized spectrum of noise produced by a low Mach number flow over a sinusoidal TE: (a) from [3] Equation (5.6); (b) Matlab Code.

\subsubsection{Optimization of single-size sawtooth serrations}

The noise spectrums for both a straight TE and each of the optimized, single-size, sawtooth TE designs are given in Figure 3.16. The optimized single-size sawtooth design, which produced the least noise, was obtained in the study of SS-Saw-1.

This study applied the largest upper limit, and the smallest lower limit on $h$ and $\lambda$ respectively compared to the other studies in the SS-Saw group. The $O A S P L_{n o r m}$ produced by SS-Saw-1 was $16.7 \mathrm{~dB}$, corresponding to a $30.1 \mathrm{~dB}$ of a straight trailing edge and the reduction in $\triangle O A S P L_{n o r m}$ is $13.1 \mathrm{~dB}$ compared to a straight trailing edge (see the Table 3.2 for more details). 


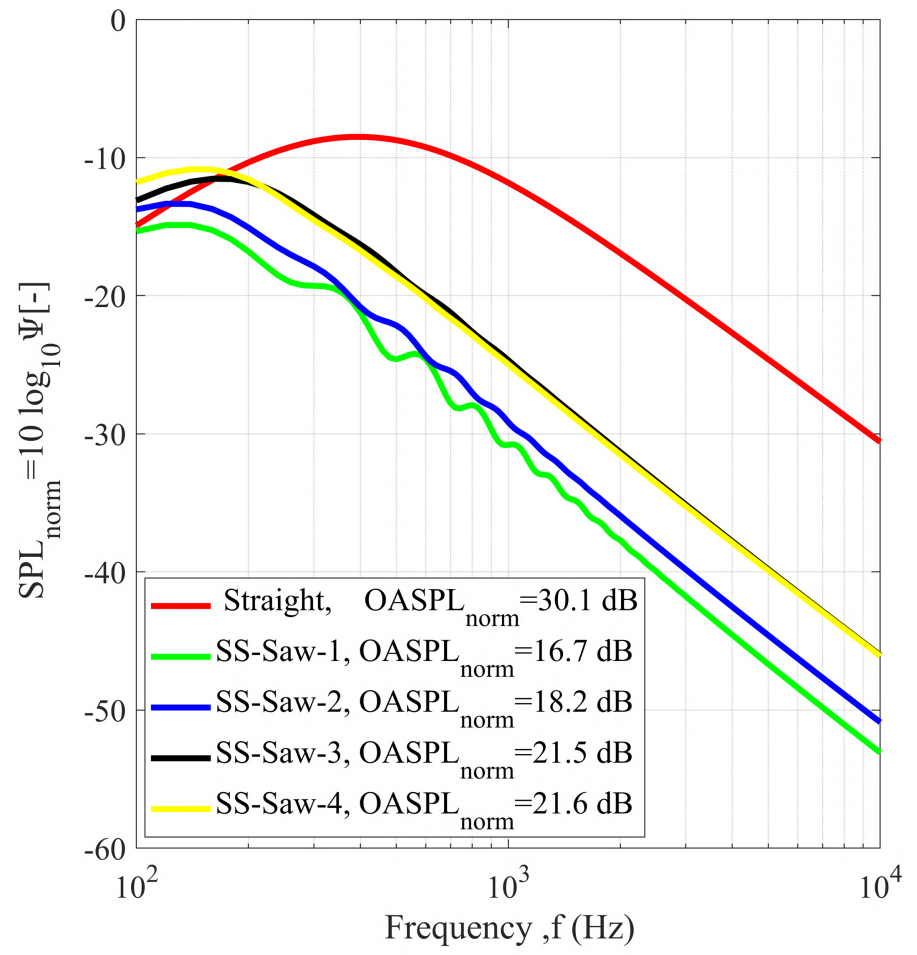

Figure 3.16: $S P L$ plotted as a function of frequency for optimum optimized sawtooth TE.

\subsubsection{Optimization of single-size slitted serrations}

The noise spectrums for both a straight TE and each of the optimized, single-size, slitted TE designs are given in Figure 3.17. Of the single-size slit optimization studies, the design that produced the least noise was SS-Slit-1, which produced an $O A S P L_{\text {norm }}$ of $27.9 \mathrm{~dB}$ corresponding to a $30.1 \mathrm{~dB}$ of a straight trailing edge and the reduction in $\triangle O A S P L_{\text {norm }}$ is $2.2 \mathrm{~dB}$ compared to a straight trailing edge (see the Table 3.2 for more details). Similar to the single-size sawtooth optimization, SS-Slit-1 also applied the smallest lower limits on widths, $\lambda_{1}$ and $\lambda_{2}$ and the upper limit on the slit amplitude, $h$, is $6.4 \mathrm{~dB}$. The optimum widths are seen to always be equal to their lower limits of $\left(\lambda_{1}\right)_{\min }$ and $\left(\lambda_{2}\right)_{\min }$. 


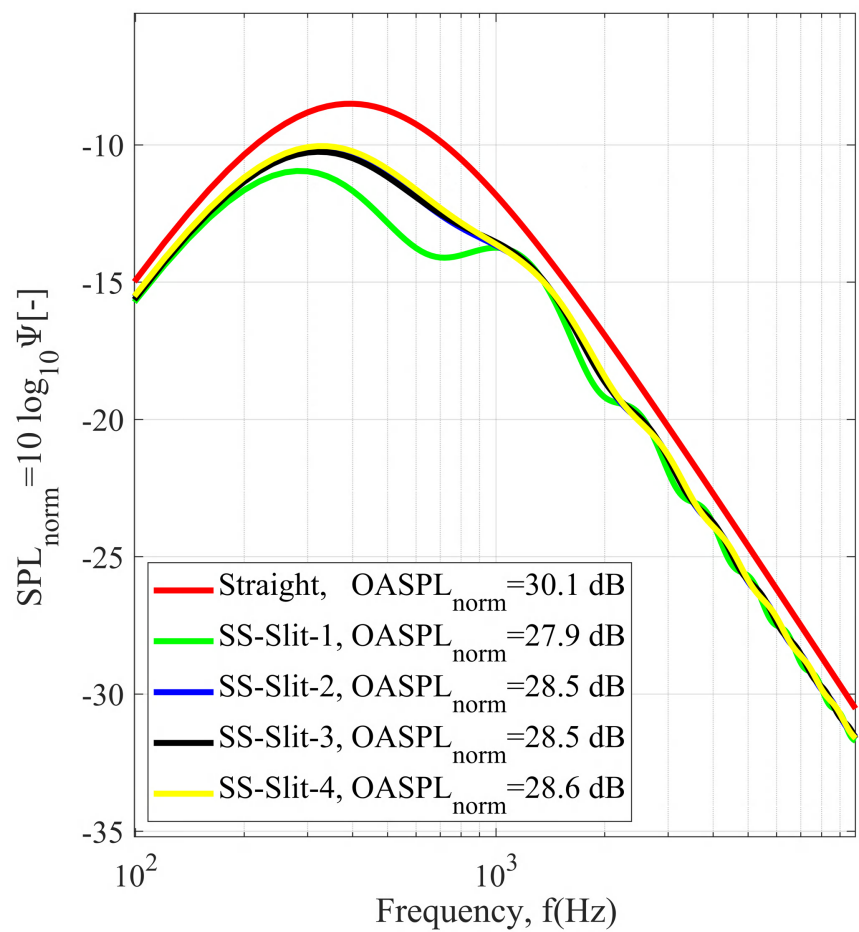

Figure 3.17: Normalized spectrum plotted as a function of frequency between $0.1 \mathrm{kHz}$ and $10 \mathrm{kHz}$ for $O A S P L_{\text {norm }}$ optimized slitted trailing edge profiles.

\subsubsection{Optimization of single-size sinusoidal serrations}

The noise spectrums for both a straight TE and each of the optimized, single-size, sinusoidal TE designs are given in Figure 3.18. The optimized single-size sinusoidal design, which produced the least noise, was obtained in the study of SS-Sinu-2.

Similar to the single-size sawtooth optimization, this study applied the largest upper limit, and the smallest lower limit on $h$, and $\lambda$ respectively compared to the other studies in the SS-Sinu group. The $O A S P L_{\text {norm }}$ produced by SS-Sinu-2 was $20.0 \mathrm{~dB}$, corresponding to a $30.1 \mathrm{~dB}$ of a straight trailing edge and the reduction in $\triangle O A S P L_{\text {norm }}$ is $10.1 \mathrm{~dB}$ compared to a straight trailing edge (see the Table 3.2 for more details). 


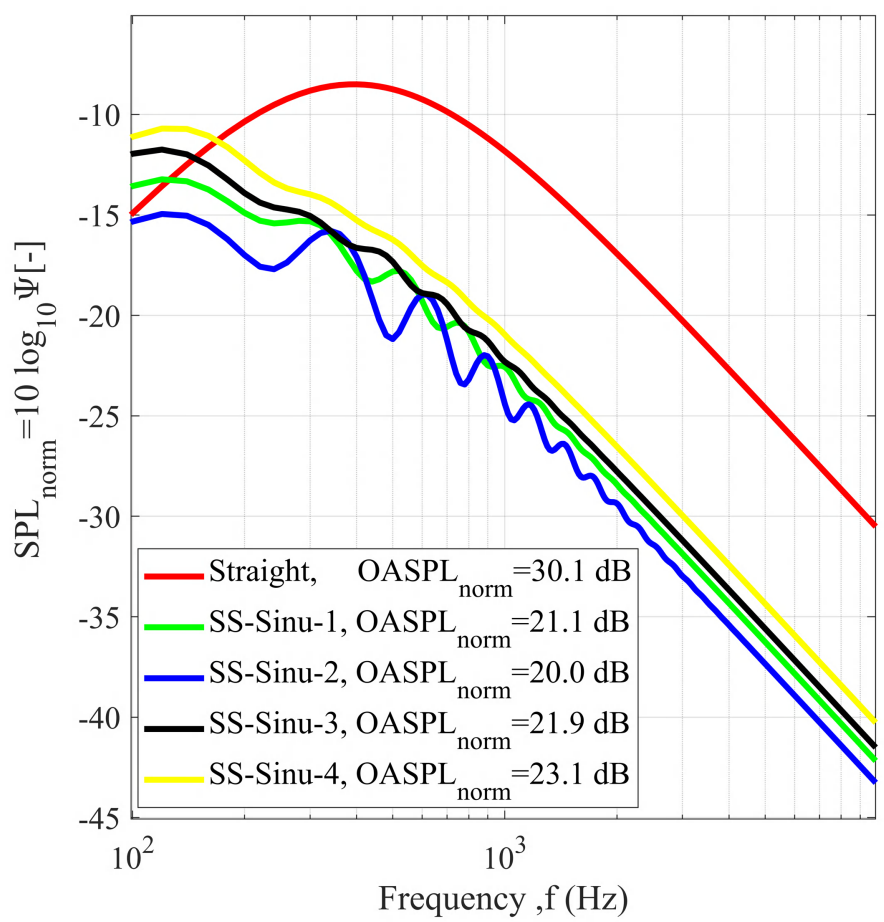

Figure 3.18: Normalized spectrum plotted as a function of frequency between $0.1 \mathrm{kHz}$ and $10 \mathrm{kHz}$ for $O A S P L_{\text {norm }}$ optimized sinusoidal trailing edge profiles.

Each of the optimum designs in all studies, (see Figure 3.19), sawtooth, slitted and sinusoidal TE produces a lower $O A S P L_{\text {norm }}$ than the straight TE; however, significant variations are seen in the amount of reduction achieved between the three, TE designs. This observation suggests that the optimization process can produce TE serration designs that produce less noise than designs that have previously been studied, and whose dimensions were chosen manually. Furthermore, based on our analysis, the sawtooth serration, SS-Saw-1, provides the greatest noise reduction, $\triangle O A S P L_{n o r m}$, over a wide range of frequencies as shown in Figure 3.19. 

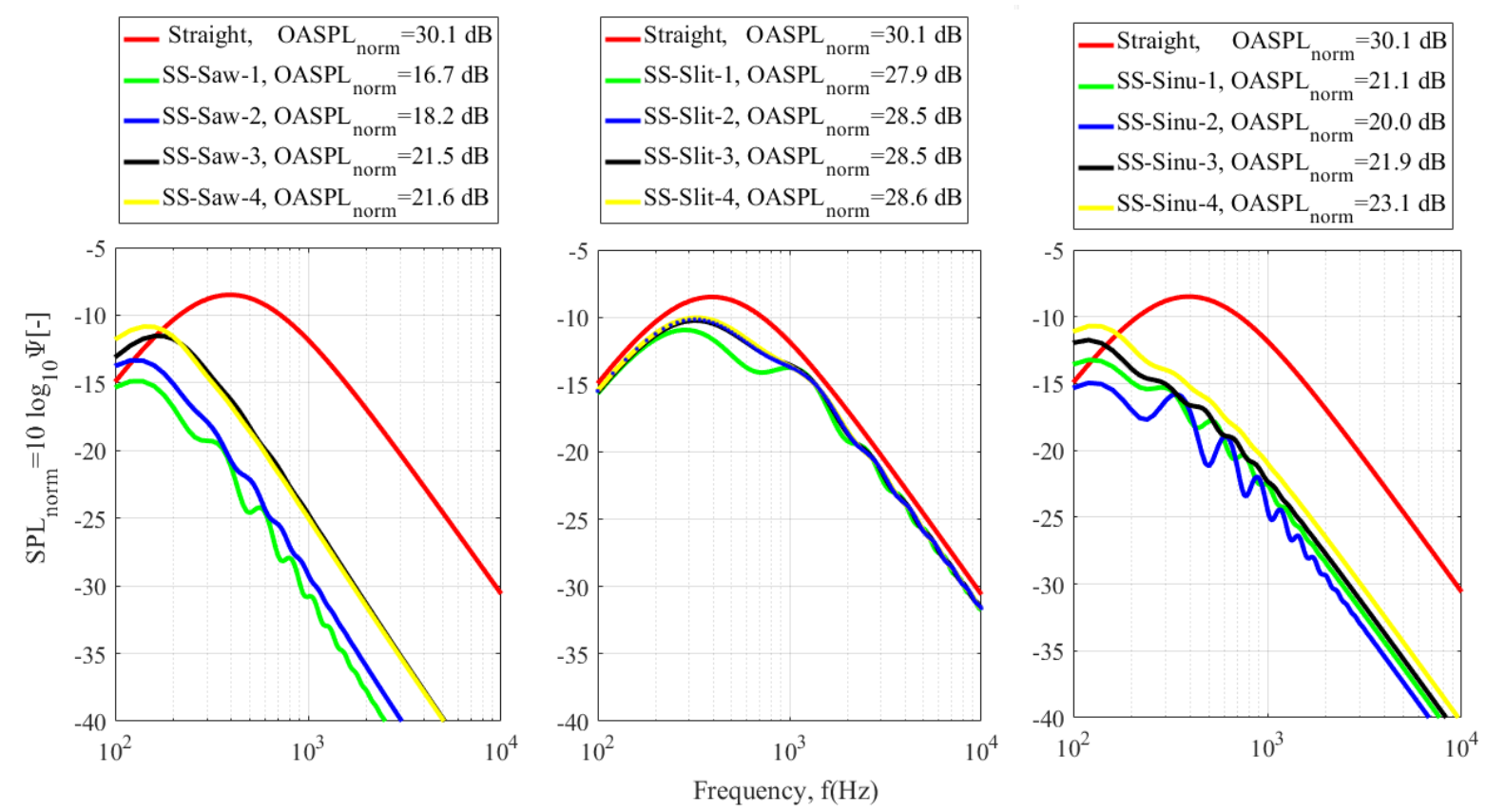

Figure 3.19: The compression between the optimum serrations.

\subsubsection{Experimental results and comparison with numerical re- sults}

A comparison of the far-field noise $S P L$ spectrum, measured at $90^{\circ}$ overhead of the airfoil and flat-plate, trailing edge, between the best optimum serrated (SS-Saw-1, SS-Slit-1 and SS-Sinu-2) and the straight trailing edge, as defined in Table 3.2, is shown in Figure 3.20 . Below $\sim 250 \mathrm{~Hz}$, an airfoil TE noise reduction relative to the straight trailing edge of between $\sim(1$ to 6$) \mathrm{dB}$ can be seen for all trailing edge treatments. 

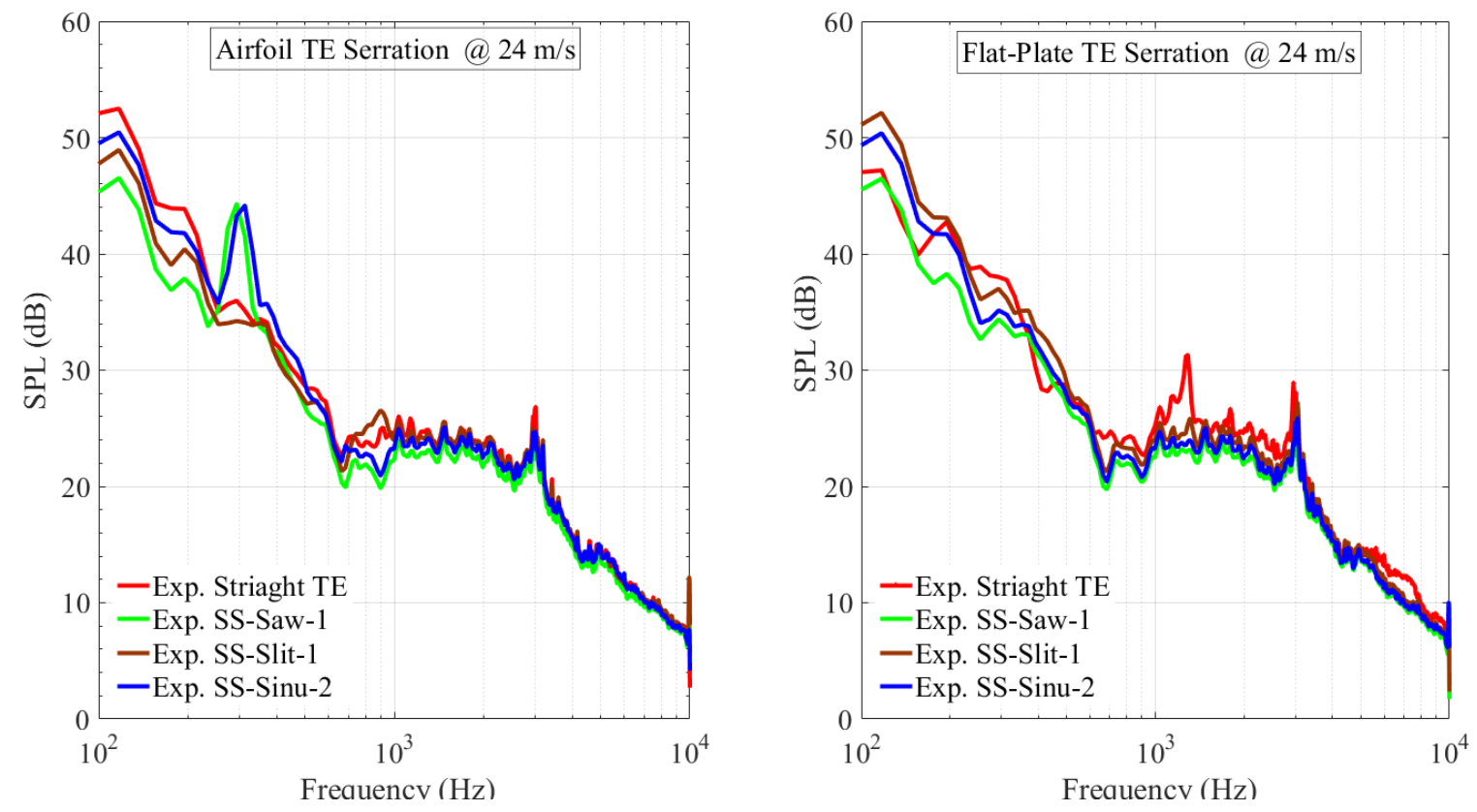

Figure 3.20: Measured far-field noise spectrum showing a comparison between straight and the sawtooth, slitted and sinusoidal TE with airfoil and flat-plat at $0^{0}$ AoA and $\mathrm{U}=24 \mathrm{~m} / \mathrm{s}$.

A comparison of the noise reduction, $\triangle S P L$, between the predicted/optimized and experimental data for various optimum serrated trailing edges is shown in Figure 3.21 . The level of predicted noise reduction is approximately $\sim(29$ to 52$)$ dB higher than the measured reduction for SS-Saw-1 and SS-Sinu-2 and approximately $\sim(1$ to 3$)$ dB for SS-Slit-1. This is in trend agreement with the previous results shown in [28] for sawtooth serrations. The predicted noise reduction tends to increase with frequency, while the measured one decreases with frequency. 

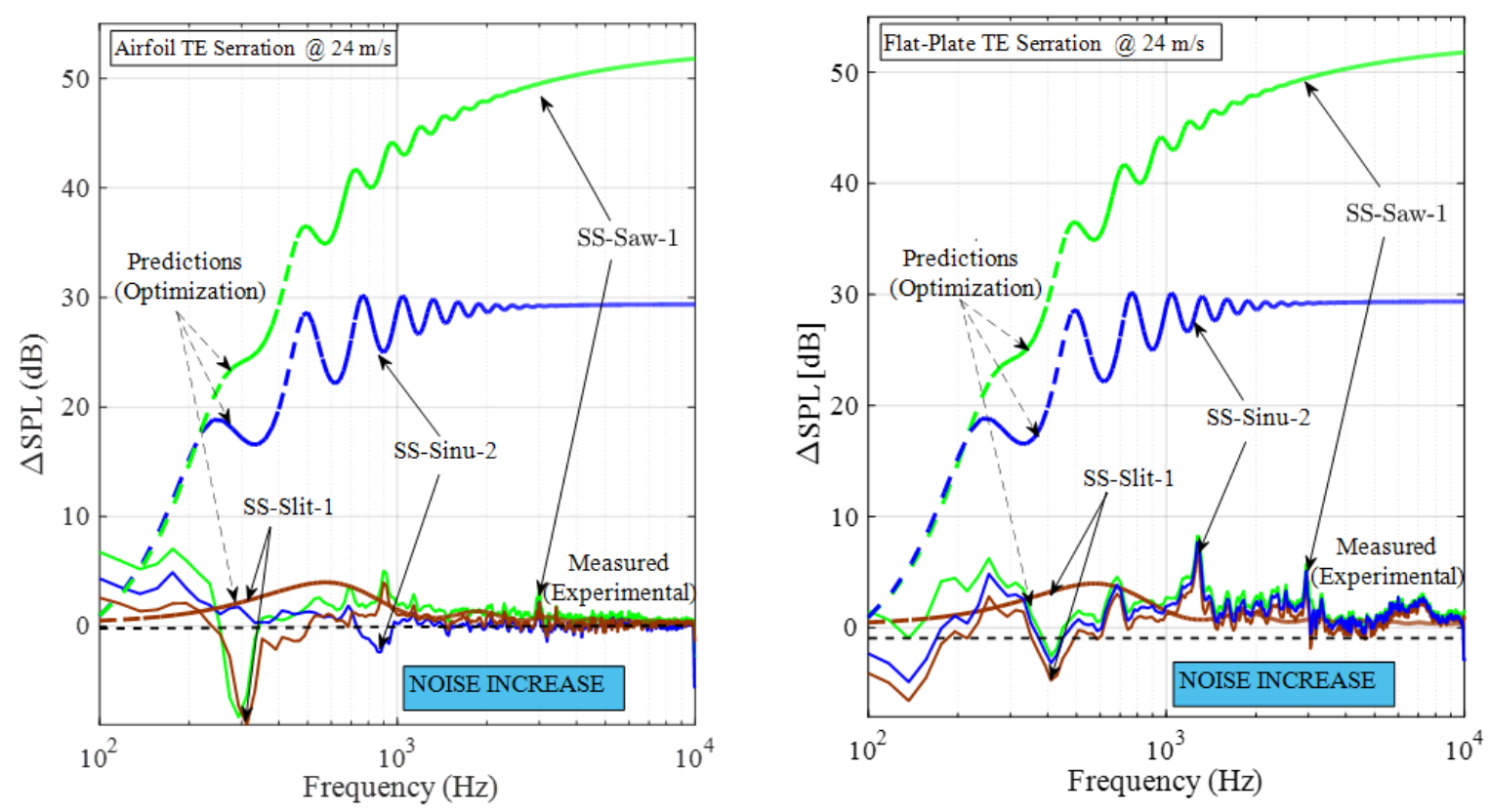

Figure 3.21: Broadband noise reduction predicted by Howe (Dashed) and measured experimentally (Solid) for serration profiles SS-Saw-1, SS-Slit-1 and SS-Sinu-2 Experimental data with airfoil and flat-plate at $0^{0} \mathrm{AoA}$ and $\mathrm{U}=24 \mathrm{~m} / \mathrm{s}$.

The strong oscillations observed in the predicted noise reduction in Figure 3.21 are due to interference between the root and the tip of the serrations, which are not observed in the experimental data. Note that the oscillations observed in the experimental spectra are characteristics of trailing edge noise and are not related to the serrated edges as described by Amiet [33]. The predicted oscillations from the optimization are a direct consequence of Howe's model simplifying assumption; i.e., that the scattering process is the predominant source of TE noise with no extraneous sources present. However, experimental results suggest that extraneous noise sources may be present due to unsteadiness close to the serrations. While the noise reduction amounts from predictions and experimental data differ, Howe's model is still very useful for the optimization work to determine the best TE configurations which reduced the noise radiated

The discrepancies observed in Figure 3.21 between the optimization and measured noise reductions for serrated trailing edges speculates on the possible reasons: 
- Assumption that the surface pressure in the boundary layer at the trailing edge is not affected by the serrations, but it is locally affected by the serration. However, this effect is likely to be small $|76|$.

- The quadrupole sources in the boundary layer of the airfoil, upstream of the trailing are not accounted for in the theoretical far-field radiation.

- Numerically shown by Jones and Sandberg [77], the unsteadiness close to the serration due to horse-shoe vortices can be a source of extraneous noise.

\subsection{Conclusion}

An aeroacoustic wind tunnel test section has been built at Carleton University. The tunnel has a rectangular test section with dimensions of $0.78 \mathrm{~m}$ in width, $0.51 \mathrm{~m}$ in height and $1.83 \mathrm{~m}$ long. Its aeroacoustic performance is measured. Results show that the background noise can be comparable with other aeroacoustic wind tunnels worldwide. A simplified airfoil and flat-plate TE noise are tested as a benchmark test. Results show that the serration geometry is effective in reducing noise and, the noise radiated from the $\mathrm{TE}$ is at least $10 \mathrm{~dB}$ higher than the background noise, satisfying the requirements for aeroacoustic measurements.

Three different geometrical profiles of trailing edge serrations have been optimized for the reduction of TBL-TE noise. The optimum width for the three, sawtooth, slitted and sinusoidal serrations, are the smallest allowable one in the overall spectrum frequencies. The optimum single-size sawtooth and sinusoidal always has the largest allowable amplitude whereas the optimum single-size slit has a specific amplitude value to optimize the balance.

Noise radiation from optimized trailing edges with different serrations, sawtooth, slitted and sinusoidal, has been investigated. It has been shown that the TE serration design 
can have a significant effect on the level of noise reduction. It has been shown that numerical and experimental results strongly suggest that optimized serrated trailing edge configurations can yield less TBL-TE noise compared to the traditional straight trailingedge configuration. Moreover, based on the optimization and experimental analyses, the sawtooth serration provides the greatest noise reduction over a wider range of frequencies when compared to slitted and sinusoidal TE serrations. 
Chapter 4

\title{
Aeroacoustic Design Optimization and
}

\section{Experimental Investigation of Sawtooth}

\section{Trailing Edges for Maximized Noise}

\section{Reduction}

\begin{abstract}
The aim of the current study is to employ a formal optimization approach and to find the optimal sawtooth shape of trailing-edge serrations that maximize the turbulent boundary layer-trailing edge (TBL-TE) interaction noise reduction. Lyu's semi-empirical model is used for the optimization approach to investigate a semi-infinite flat plate at zeroangle of attache and low Mach Number. The ability of sawtooth trailing-edge serrations to reduce TBL-TE noise is analyzed through a single-objective numerical optimization study, and trailing-edges tested in a wind tunnel. Four different cases of sawtooth serration geometries are tested for NACA-0012 airfoil and flat-plate trailing edges. Results from the optimization study are used to examine and validate the influence of serration
\end{abstract}


design parameters. For all the cases, the optimum size shows that the maximum noise reduction is located in regions of the design space where the amplitude is maximal and the wavelength is minimal. Moreover, based on the optimization analyses, the Saw-1 serration provides the greatest noise reduction up to $12.89 \mathrm{~dB}$ over a wider range of frequencies. Experimental and optimization results are then compared. It is concluded that numerical and experimental results agree that optimized sawtooth trailing-edge configurations can yield less TBL-TE noise compared to the traditional straight-trailing-edge configuration. The optimization studies underestimated the measurements by about $\sim(2$ to 12$) \mathrm{dB}$, but the tendency between the different serration geometries was predicted correctly.

\subsection{Introduction}

Aircraft noise was considered a problem throughout the $20^{\text {th }}$ century because it is a nuisance $[13 \mid$. At the turn of the century, the problem became larger when considering trends in the aerospace industry that include increasing aircraft size and speed as well as major increases in the volume of air traffic [78]. During the aircraft landing, while the engine throttle is turned down significantly, an important source of noise arises from the turbulent boundary layers (TBL) flow-structures convecting downstream across solid surfaces and interacting with the trailing edges. This mechanism of noise generation is known as the turbulent boundary layer-trailing-edge (TBL-TE) noise, and its frequency spectrum is broadband in nature. TBL-TE noise is also a major source of noise in other applications, such as from wind turbines where exposure to nearby communities has been a complaint.

According to airframe noise studies, more work should be done on noise generation mechanisms and noise reduction approaches $[30,79,80]$. One promising method of re-

ducing TBL-TE noise is the addition of a flat-plate, serrated trailing-edge extensions. 
This method of noise reduction has long been regarded viable, but in the recent decade, it has garnered more attention. Experiments, theoretical investigations, and numerical studies [28, 35, 36, 45, 61 have all demonstrated the potential for trailing-edge serrations to minimize TBL-TE noise. Although the amount of noise reduction and frequency ranges in which noise attenuation occurs varies between studies, it is well accepted that trailing-edge serrations can reduce TBL-TE noise for at least some parts of the frequency spectrum [62].

TBL-TE noise reduction has previously been investigated through optimization studies 44,63 , although these have mostly focused on changing the airfoil shape and profile itself around the area near the trailing-edge, rather than investigating optimized serrations. These studies were able to demonstrate that new, lower-noise designs could be created through numerical optimization processes by using a semi-empirical model of noise prediction. General tendency have been extrapolated from research including the prediction of TBL-TE noise from serrated trailing-edges.

The present study presents a formal optimization study to find the optimal sawtooth shape for trailing edge serrations that maximized noise reduction. While previous optimization studies have used the original Howe's model [28], the current approach implements Lyu's model, which is an improved and more accurate model. The far-field sound pressure emitted from a NACA-0012 airfoil was obtained in wind tunnel. Four sawtooth TEs geometries were tested on the airfoil and also a flat plate for comparison. The results from the optimization study were then compared and validated with wind tunnel measurements.

\subsection{Sawtooth TE Sound Model}

The model used in this section is an extension of Howe's model [36], developed by Lyu et al. [5. Lyu's model focused specifically on sawtooth serrations, which has been shown to be more effective in reducing the trailing-edge noise than the sinusoidal and slitted [28]. As shown in Figuer 4.1, consider an airfoil with sawtooth trailing-edge, modeled as a flat- 
plate. The following assumptions are applied :

- The flat-plate is considered as semi-infinite, zero thickness flat-plate at $0^{0}$ angle of attack;

- The flat-plate and the trailing-edge serrations are periodic in the spanwise direction;

- The span is considered to be much larger than the spanwise correlation length of the boundary-layer turbulence;

- The flow is represented by its mean component convecting an incident gust and the gust is described by a two-dimensional spectrum $P_{0} e^{\left(-i\left(\omega t-k_{1} x_{1}-k_{2} x_{2}\right)\right)}$, where $P_{0}$ is the gust amplitude and $k_{1}, k_{3}$ are the streamwise and spanwise wavenumbers;

- The turbulence in the incoming boundary layer is assumed to be frozen;

- The flat-plate is positioned in the global coordinate system where $\mathrm{x}$ is the streamwise, $\mathrm{y}$ the spanwise coordinates and, $\mathrm{z}$ the wall-normal.

Figure 4.1 presents a sketch of a serrated trailing-edge and the related local coordinates system. The first step is to solve for pressure scattered at the trailing-edge with Schwartzschild's technique 81,82. 


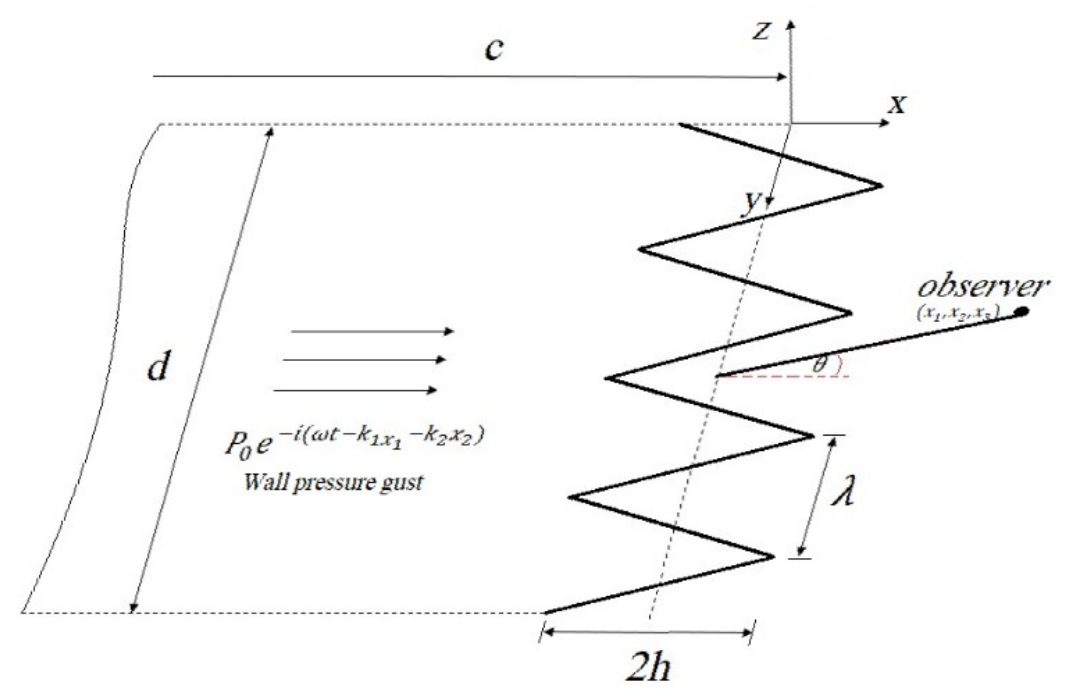

Figure 4.1: Flat-plate with serrated trailing-edge and the related local coordinates system.

To solve the scattered pressure field on the surface of a non-rotating thin flat-plate airfoil for an incident skewed gust $P_{0} e^{\left(-i\left(\omega t-k_{1} x_{1}-k_{2} x_{2}\right)\right)}$ (see Figure 4.1) convected at velocity $U_{c}$, a Fourier expansion in the spanwise direction is introduced. This leads leads to an inhomogeneous partial differential equation. Lyu et al. [5] solved the inhomogeneous partial deferential equation (PDE) with an iterative process. The far-field sound pressure at the observer position $\mathrm{x}$ induced by the scattered surface pressure is calculated using the surface pressure integral and is expressed by

$$
P_{f}\left(x, \omega, k_{2}\right)=P_{0}\left(\frac{-i \omega x_{3} c}{4 \pi c_{0} S_{0}^{2}}\right) \lambda \frac{\sin \left((N+1 / 2) \lambda\left(k_{2}-k x_{2} / S_{0}\right)\right)}{\sin \left(\left(k_{2}-k x_{2} / S_{0}\right) \lambda / 2\right)} \mathcal{L}\left(\omega, k_{1}, k_{2}\right)
$$

where $P_{0}$ is the magnitude of the incident wall pressure gust, $k=\sqrt{\left(x / c_{0}\right)}$ is the acoustic wavenumber, $S_{0}=\sqrt{\left(x_{1}^{2}+\beta^{2}\left(x_{2}^{2}+x_{3}^{2}\right)\right.}$ is the corrected distance for convection effects and $(2 N+1)$ is the number of sawtooth on the trailing-edge.

$\mathcal{L}$ is the far-field sound gust response function defined as 


$$
\mathcal{L}\left(\omega, k_{1}, k_{2}\right)=(1-i) \frac{1}{\lambda c} e^{-i k\left(M_{0} \chi_{1}-S_{0}\right) / \beta^{2}} e^{i k\left(M_{0}-\chi_{1} / S_{0}\right) h / \beta^{2}} \sum_{n=-\infty}^{\infty}\left(\Theta_{n}+\Theta_{n}^{(1)}+\Theta_{n}^{(2)}+\ldots\right),
$$

where $k_{1}=\omega / U_{c}$, is the convective wave number in stream-wise direction and $\Theta_{n}^{(0)}$ and $\Theta_{n}^{(1)}$ denote the radiation function of order 0 and 1, respectively as defined

$$
\begin{gathered}
\Theta_{n}^{(0)}=a_{n} Q_{m n} \\
\Theta_{n}^{(1)}=\sum_{m=-\infty}^{\infty}\left(\nu_{n m} a_{m}\left(i k_{1}\left[Q_{n n}-Q_{n m}\right]-\sqrt{\mu_{m}}\left[S_{n n}-S_{n m}\right]\right)\right.
\end{gathered}
$$

for the second order $\Theta^{(2)}$, radiation function and the definition of the subfunctions are detailed in Lyu et al. [5].

The function $Q_{m n}$ and $S_{m n}$ in the above equation are given by

$$
\begin{aligned}
Q_{m n}= & \sum_{j=0}^{1} \frac{1}{\kappa_{n j}}\left(\frac{1}{\mu_{A}}\left[e^{i \kappa n j \chi_{j+1}} \Gamma\left(c+\epsilon_{j+1} ; \mu_{m}, \mu_{A}\right)-e^{i \kappa_{n j} \chi_{j}} \Gamma\left(c+\epsilon_{j} ; \mu_{m}, \mu_{A}\right)\right]\right. \\
& \left.-\frac{1}{\mu_{B n j}} e^{i \kappa_{n j}\left(\chi_{j}-\left(c+\epsilon_{j}\right) / \sigma_{j}\right)}\left[\Gamma\left(c+\epsilon_{j+1} ; \mu_{m}, \mu_{B n j}\right)-\Gamma\left(c+\epsilon_{j} ; \mu_{m}, \mu_{B n j}\right)\right]\right), \\
S_{m n}= & \sum_{j=0}^{1} \frac{1}{i \kappa_{n j}}\left(\frac{1}{\sqrt{\eta_{A m}}}\left[e^{i \kappa_{n j} \chi_{j+1}} E\left(\eta_{A m}\left(c+\epsilon_{j+1}\right)\right)-e^{i \kappa_{n j} \chi_{j}} E\left(\eta_{A m}\left(c+\epsilon_{j}\right)\right)\right]\right. \\
& \left.-\frac{1}{\sqrt{\eta_{B m j}}} e^{i \kappa_{n j}\left(\chi_{j}-\left(c+\epsilon_{j}\right) / \sigma_{j}\right.}\left[E\left(\eta_{B m j}\left(c+\epsilon_{j+1}\right)-E\left(\eta_{B m j}\left(c+\epsilon_{j}\right)\right)\right)\right]\right)
\end{aligned}
$$


where the function $\Gamma$ is defined by

$$
\Gamma(c ; \mu, \nu)=e^{-i v c} E(\mu c)-\sqrt{\frac{\mu}{\mu-\nu}} E((\mu-\nu) c)-\frac{e^{-\nu c}}{1-i}
$$

and

$$
\left\{\begin{array}{l}
\mu_{A}=k_{1}+k\left(M_{0}-x_{1} / S_{0}\right) / \beta^{2}, \\
\mu_{B n j}=k_{1}-\left(k_{2 n}-k x_{2} / S_{0}\right) \sigma_{j}, \\
\kappa_{n j}=k_{2 n}-k x_{2} / S_{0}+k\left(M_{0}-x_{1} / S_{0}\right) \sigma_{j} / \beta^{2}, \\
\eta_{A m}=K_{m}+k M_{0} /\left(\beta^{2}+\sigma^{2}\right)-k\left(M_{0}-x_{1} / S_{0}\right) / \beta^{2}, \\
\eta_{B n j}=K_{m}+k M_{0} /\left(\beta^{2}+\sigma^{2}\right)+\left(k_{2 n}-k x_{2} / S_{0}\right) / \sigma j . \\
k_{2 n}=k_{2}+n \frac{2 \pi}{\lambda}
\end{array}\right.
$$

where $k_{2 n}$ is the wave number in span-wise direction of Fourier mode $n$.

Using the far-field sound pressure of equation (4.1), the power spectral density (PSD) of the far-field sound in the plane $x_{2}=0$ is given by

$$
S_{p p}(x, \omega)=\left(\frac{\omega x_{3} c}{4 \pi c_{0} S_{0}^{2}}\right)^{2} 2 \pi d \sum_{m=-\infty}^{\infty}\left|\mathcal{L}\left(\omega, k_{1}, 2 m \pi / \lambda\right)\right|^{2} \Pi(\omega, 2 m \pi / \lambda)
$$

where $k_{1}=\omega / U_{c}$, is the chordwise wavenumber and $\Pi\left(\omega, k_{2}\right)$ is the wavenumber spectral density of the surface pressure beneath the turbulent boundary layer on the airfoil surface. It is approximated by

$$
\Pi\left(\omega, k_{2}\right) \approx \frac{4 C_{m} \rho_{0}^{2} v_{*}^{4}\left(\omega / U_{c}\right)^{2} \delta^{4}}{U_{c}\left(\left(\left(\omega / U_{c}\right)^{2}+k_{2}^{2}\right) \delta^{2}+\chi^{2}\right)^{2}}
$$


where $\rho_{0}$ and $\delta$ are the fluid density and the boundary layer thickness respectively, $C_{m} \approx 0.1553, \chi=1.33$ and $v_{*}=0.03 U_{0}$. The convection velocity is taken as $U_{c}=0.7 U_{0}$ with $U_{0}$ the flow velocity.

By substituting 4.11 into 4.10 and using $\left(\rho_{0} u_{*}^{2}\right)^{2}\left(d / c_{0}\right)$ to non-dimensional the farfield PSD we obtain

$$
S_{p p}(x, \omega)=\left(\rho_{0} u_{*}^{2}\right)^{2}\left(\frac{d}{c_{0}}\right)\left(\frac{C_{m}}{2 \pi}\right) \Psi(\omega)
$$

The final form of the normalized sound pressure spectrum, $\Psi(\omega)$, for sawtooth trailingedge geometry is defined as

$$
\Psi(\omega)=\left(\frac{x_{3} c}{S_{0}^{2}}\right)^{2}\left(\frac{U_{c}}{c_{0}}\right) \sum_{m=-\infty}^{\infty}\left|\mathcal{L}\left(\omega, k_{1}, 2 \pi m / \lambda\right)\right|^{2} \frac{\left(\omega \delta / U_{c}\right)^{4}}{\left[\left(\omega \delta / U_{c}\right)^{2}+(2 m \pi \delta / \lambda)^{2}+\chi^{2}\right]^{2}}
$$

The boundary layer thickness, $\delta$, at the airfoil trailing-edge is an important parameter in determining the noise performance of trailing-edge serrations. The TBL thickness used in the noise prediction is calculated as following $\mid 72,83$ :

$$
\delta=\frac{0.37 c\left[1+\left(\frac{R e_{c}}{6.9 \times 10^{7}}\right)^{2}\right]^{1 / 10}}{\operatorname{Re}_{c}^{\frac{1}{5}}}
$$

The non-dimensional normalized overall sound pressure level $(O A S P L)$ describes the total amount of noise produced across a desired frequency range. The $O A S P L$ is used both as an objective function, and for the comparison of various trailing-edge designs to a straight trailing edge. The $O A S P L$ is calculated as follows:

$$
O A S P L=10 \log _{10}\left(\int_{\omega_{\min }}^{\omega_{\max }} \Psi(\omega) d \omega\right)
$$

and 


$$
\Delta O A S P L=10 \log _{10}\left(\int_{\omega_{\min }}^{\omega_{\max }}\left(\frac{\Psi_{0}(\omega)}{\Psi(\omega)}\right) d \omega\right)
$$

where $\omega_{\min }$ and $\omega_{\max }$ are the lower and upper bounds on the frequency range of interest respectively and, $\Psi(\omega)$ can be obtained from equation (4.13) according to the sawtooth geometry and $\Psi_{0}(\omega)$ is the sound spectrum level from the straight trailing-edge geometry.

\subsection{Numerical Optimization Method}

Investors use optimization to create portfolios that avoid excessive risk while achieving a high rate of return. On the other hand, manufacturers aim for maximum efficiency in the design and operation of their production processes. In engineering, the goal of optimization is to adjust parameters to optimize the performance of their designs to make sure that a design performs as effectively and efficiently as possible [84.

Optimization is an essential tool in decision science and in the analysis of physical systems. To make use of this tool, first an objective has to be identified, i.e., a quantitative measure of the performance of the system under study. This objective could be profit, time, noise, potential energy, or any quantity or combination of quantities that can be represented by a single number. The objective depends on certain characteristics of the system, called variables or unknowns. The goal is to find values of the variables that optimize the objective. Often the variables are restricted, or constrained, in some way.

\subsubsection{Definition of the optimization problem}

The optimization code developed for this study is based on an add-on to Matlab. It uses an interior point algorithm for a nonlinear equation. The equations being analyzed are 
nonlinear functions; therefore, no linear optimization equation could be used. An interior point algorithm is an approach to constrained minimization by solving a sequence of approximate minimization problems. The name interior point method means it lies in the interior of the feasible region. This is different from the simplex method which moves its iterations along the boundary of the feasible region from one extreme point to another [85]. In the past 30 years there has been significant advancement in interior point methods after the work of Karmarkar in 1984 [86]. Many books have been written explaining the basics of the method and the applications it has for both linear and nonlinear functions 85, 87, 88. This type of algorithm can be used in many types of applications [88 90], and therefore it was selected to obtain initial optimized design variables.

This study examines one optimization problem, which is given below in proper form in equation 4.17). This equation corresponds to the single-size optimization of sawtooth TE geometry (see Figure 4.1). In each case, the optimum design is the single-size of serration that produces the least amount of total noise overall frequencies between 0.1 $\mathrm{kHz}$ and $10 \mathrm{kHz}$.

$$
\left\{\begin{array}{cc}
\text { minimize } & Z_{\text {saw }}=O A S P L(h, \lambda) \\
h, \lambda & \\
\text { subject to } & h_{\min } \leq h \leq h_{\max } \\
& \lambda_{\min } \leq \lambda \leq \lambda_{\max }
\end{array}\right.
$$

where $Z_{\text {saw }}$ is the objective function value; $h_{\min }$ and $h_{\max }$ are the smallest and largest feasible serration amplitudes corresponding to the sawtooth geometry, $\left(\lambda_{\min }\right)$ and $\left(\lambda_{\max }\right)$ are the smallest and largest feasible widths. The values of lower and upper design variables were chosen manually. A summary of the optimization studies setting is given in Table 4.1. 
Table 4.1: Optimization trials and associated design variable bounds $(U=24 \mathrm{~m} / \mathrm{s})$.

\begin{tabular}{cccccc}
\hline \multirow{2}{*}{ Study No } & Geometry & \multicolumn{2}{c}{ Lower DV Bound $(\mathrm{mm})$} & \multicolumn{2}{c}{ Upper DV Bound $(\mathrm{mm})$} \\
\cline { 3 - 6 } & & $\lambda_{\min }$ & $h_{\min }$ & $\lambda_{\max }$ & $h_{\max }$ \\
\hline Saw-1 & Sawtooth & 10 & 0 & 30 & 35 \\
\hline Saw-2 & Sawtooth & 15 & 0 & 35 & 40 \\
\hline Saw-3 & Sawtooth & 20 & 0 & 40 & 30 \\
\hline Saw-4 & Sawtooth & 25 & 0 & 35 & 37.5 \\
\hline
\end{tabular}

\subsection{Optimization Results}

This section presents the optimization results from the analyses for the sawtooth geometries. In addition, the far-field acoustic spectrum results are presented for different sawtooth geometrical parameters over a range of frequencies from $0.1 \mathrm{kHz}$ to $10 \mathrm{kHz}$. The possibility for noise reduction using each serration configuration is discussed, and general validation of the numerical optimization is accomplished through comparison with experimental measurements. The serrations design is optimized to find the single-size of each sawtooth geometry that produces the least noise over the entire frequency spectrum of interest $(0.1 \mathrm{kHz}$ to $10 \mathrm{kHz})$ for the set of constraints examined.

\subsubsection{Noise model verification}

The noise model previously defined in equation 4.13) was coded in Matlab to be used. The Matlab code was verified by comparing the noise prediction from the function output to the results published by Lyu et al. [5] for equation 3.6. Based on this analysis, it was concluded that the Matlab code produces identical results to the analytical equivalent, as shown in Figure 4.2 . 


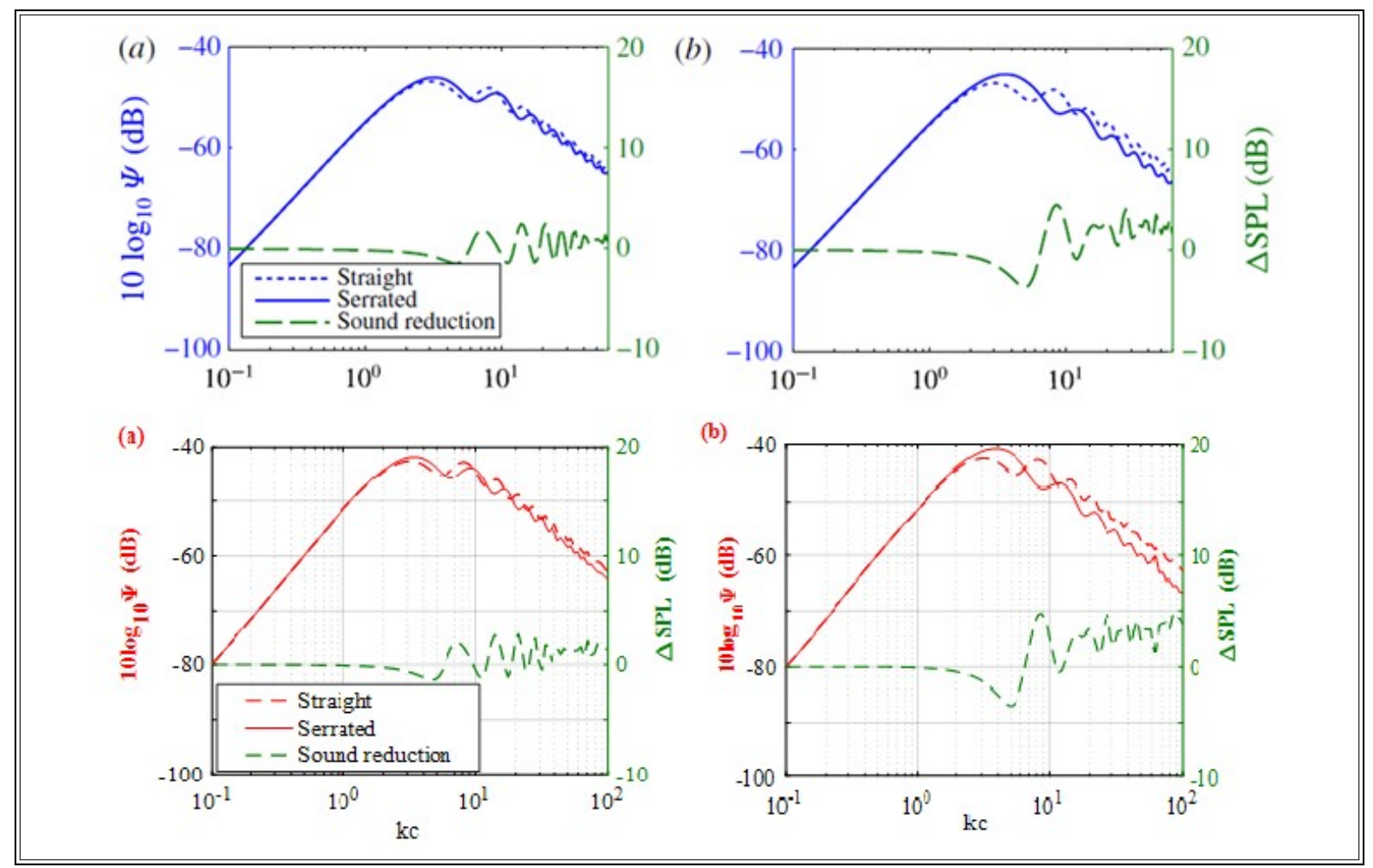

Figure 4.2: Normalized spectrum of noise produced by a low Mach number flow over a sawtooth TE: (top (a) and (b) ) from Lyu et al. [5], equation (3.6) ;(bottom (a) and (b)) Matlab code.

\subsubsection{Optimization of sawtooth serrations}

The serrations are optimized to find the single-size of sawtooth geometry that produces the least noise over the entire frequency spectrum of interest $(0.1 \mathrm{kHz}$ to $10 \mathrm{kHz})$, for the set of constraints examined. Throughout of the optimization study, the parameters in equation 4.13 which is part of the objective function definition pertaining to fluid flow $(\delta$ and $\left.U_{c}\right)$ are kept constant, while the geometrical parameters $(h, \lambda)$ are the design variables and are therefore manipulated by Matlab code to find the minimum. The choice of values for the design variables is based on airfoil dimensions (e.g. $T E_{\max }=80 \mathrm{~mm}$ ) and on the ability for properly manufacturing the parts at Carletion University. The mean flow speed was chosen to be $24 \mathrm{~m} / \mathrm{s}$ as this is representative of aircraft landing conditions or the wind turbine blade speed. The parameters used are shown in Table.4.2. 
A summary of the optimized designs from each study, and their respective $O A S P L$, are given in the Table 4.3. The $O A S P L$ for a straight trailing-edge is given in the first row of the table for comparison.

Table 4.2: The parameters used in the solution.

\begin{tabular}{lccccc}
\hline The Model & $\begin{array}{c}\text { Density } \\
\left(\mathrm{kg} / \mathrm{m}^{3}\right)\end{array}$ & $\begin{array}{c}\text { Dynamic } \\
\text { viscosity } \\
(\mathrm{kg} / \mathrm{m} . \mathrm{s})\end{array}$ & $\begin{array}{c}\text { Mach } \\
\text { number } \\
M_{0}\end{array}$ & $\begin{array}{c}\text { Reynolds } \\
\text { number } \\
(\mathrm{Rec})\end{array}$ & $\begin{array}{c}\text { Observer } \\
\text { distance- } \\
\text { to- TE }\end{array}$ \\
\hline $\begin{array}{l}\text { Airfoil and } \\
\text { Flat-plate }\end{array}$ & 1.28 & $1.72 \times 10^{5}$ & 0.04 & $5 \times 10^{5}$ & 1.4 \\
\hline
\end{tabular}

Table 4.3: Optimized designs from sawtooth study and noise produced by their trailingedge.

\begin{tabular}{|c|c|c|c|c|c|}
\hline \multirow[t]{2}{*}{ Study No } & \multicolumn{2}{|c|}{$\begin{array}{l}\text { Optimal Geometry } \\
\qquad(\mathrm{mm})\end{array}$} & \multirow[t]{2}{*}{$\begin{array}{c}O A S P L \\
{[\mathrm{~dB}]}\end{array}$} & \multirow[t]{2}{*}{$\begin{array}{l}\int_{\omega_{1}}^{\omega_{2}} \Psi_{i}= \\
10^{\frac{O A S P}{10}}\end{array}$} & \multirow[t]{2}{*}{$\begin{array}{c}\triangle O A S P L \\
{[\mathrm{~dB}]}\end{array}$} \\
\hline & $\lambda_{o p t}$ & $h_{\text {opt }}$ & & & \\
\hline Straight TE & - & - & 57.34 & 542000.89 & - \\
\hline Saw-1 & 10 & 35 & 44.59 & 28773.98 & 12.89 \\
\hline Saw-2 & 15 & 40 & 45.68 & 36982.81 & 11.66 \\
\hline Saw-3 & 20 & 30 & 48.20 & 66069.34 & 9.14 \\
\hline Saw-4 & 25 & 37.5 & 48.67 & 73620.70 & 8.67 \\
\hline
\end{tabular}

Figure 4.3 shows the normalized sound pressure level $S P L_{\text {norm }}=10 \log _{10}(\Psi)$ and the corresponding noise reduction $\triangle S P L=10 \log _{10}\left(\Psi_{0} / \Psi_{\text {saw }}\right)$ for the baseline and sawtooth configurations at $\mathrm{U}=24 \mathrm{~m} / \mathrm{s}$. Here, the convention is to use a positive $\triangle S P L$ to represent 
noise reduction, and vice versa. The dimensional frequency is used for the horizontal axes to enable an easier comparison with the experimental results. The noise spectrum results for both a straight TE and each of the optimized single-size sawtooth TE designs are shown. The straight TE noise level increases with frequency up to a maximum value (the 'hump') occurring in the frequency range $\sim 0.5 \mathrm{kHz}$ and then decays with increasing frequency in the upper mid to high-frequency range. Since the same distribution of turbulent energy in the TBL is assumed to occur regardless of the TE geometry, the spectrum of all sawtooth serration designs follows a similar, but not exact, shape to the straight TE (increase, maxima, decay). The deviations between the straight and serrated TE spectrum occur due to a fluid-structure coupling. 


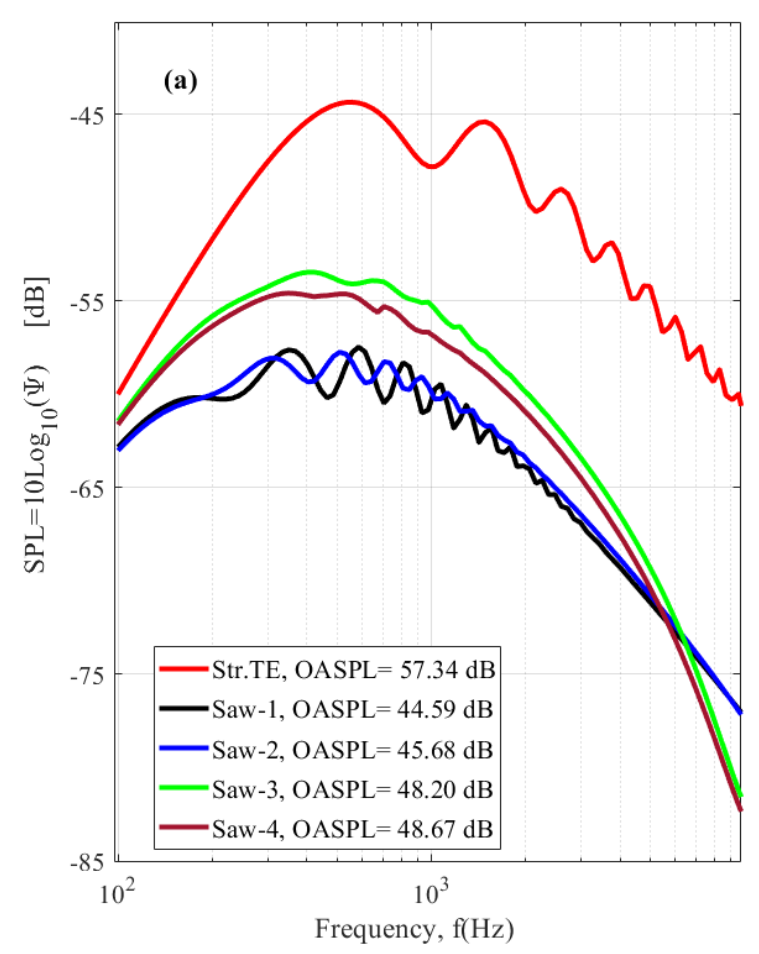

(a)

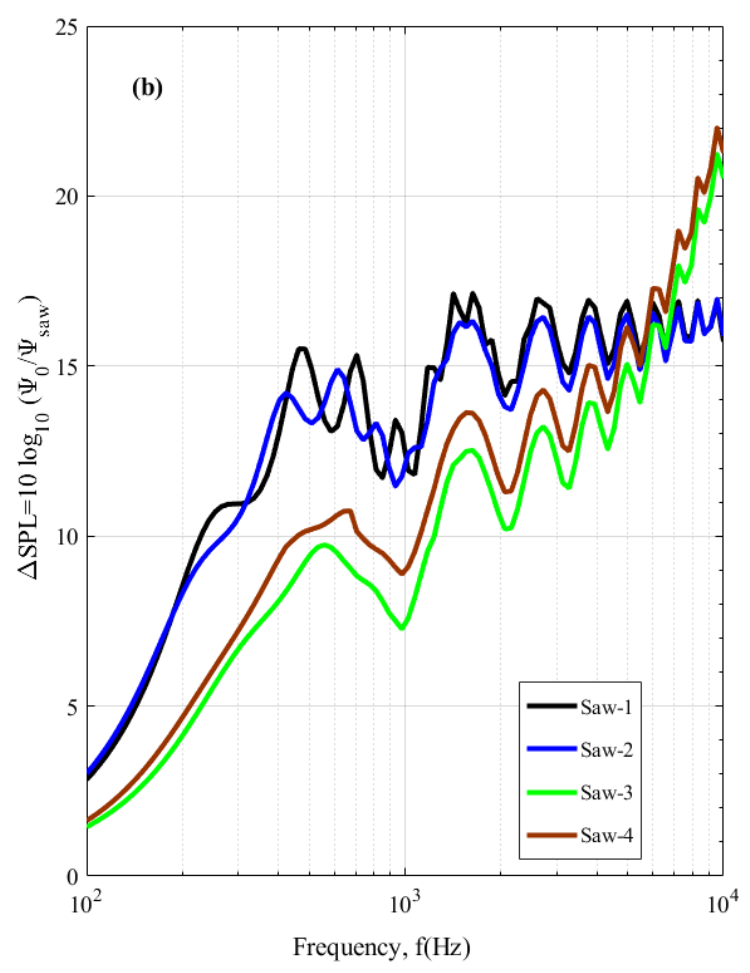

(b)

Figure 4.3: Predicted non-dimensional far-field noise $S P L_{\text {norm }}$, (a), and corresponding noise reduction $\triangle S P L_{\text {norm }}$, (b), by different sawtooth serrations according to the optimization study at $\mathrm{U}=24 \mathrm{~m} / \mathrm{s}$. A positive $\triangle S P L_{n o r m}$ denotes noise reduction.

The optimized single-size sawtooth design, which produced the least noise, was obtained in study Saw-1. This study applied the largest upper limit, and the smallest lower limit on $h$ and $\lambda$, respectively. The theoretical $O A S P L_{n o r m}$ produced by Saw-1 is 44.59 $\mathrm{dB}$, corresponding to a $57.34 \mathrm{~dB}$ of a straight trailing-edge, which corresponds to a theoretical reduction $\triangle O A S P L_{\text {norm }}$ of $12.98 \mathrm{~dB}$ (see Table 4.3 for more details). Each of the designs in studies Saw-1 to Saw-4 produce a lower $O A S P L_{\text {norm }}$ than the straight TE; however, significant variations are seen in the amount of reduction achieved between the four single-size sawtooth TE designs. This observation suggests that the optimization 
process is able to produce TE serration designs that produce less noise than designs that have previously been studied [28].

\subsection{Experimental Setup}

Section 4.4 shows that the use of the optimum sawtooth serration Saw-1 can provide more noise reduction than other TEs configuration. The goal of this section is to experimentally validate the theoretical results.

\subsubsection{The wind tunnel}

The experiments were conducted in the medium-speed, subsonic, closed-loop wind tunnel at Carleton University (as shown in Figure 4.4). The airflow is powered by a 37.3 $\mathrm{kW}(50 \mathrm{HP}$ ) variable-speed DC motor driving a $1.2 \mathrm{~m}$ axial propeller at speeds as high as 900 RPM. A variable frequency drive (VFD) modulates the rotational frequency of the fan at a resolution of $1.0 \mathrm{~Hz}$. A series of turbulence grids precede a 9:1 contraction, which reduces the turbulence intensity levels in the center of the test section to less than $0.27 \%$. The tunnel has a removable, rectangular aeroacoustic test section measuring $1.83 \mathrm{~m}$ in

length and $0.78 \mathrm{~m} \times 0.51 \mathrm{~m}$ at the inlet in width and height, respectively (See 28,29 for more details). 


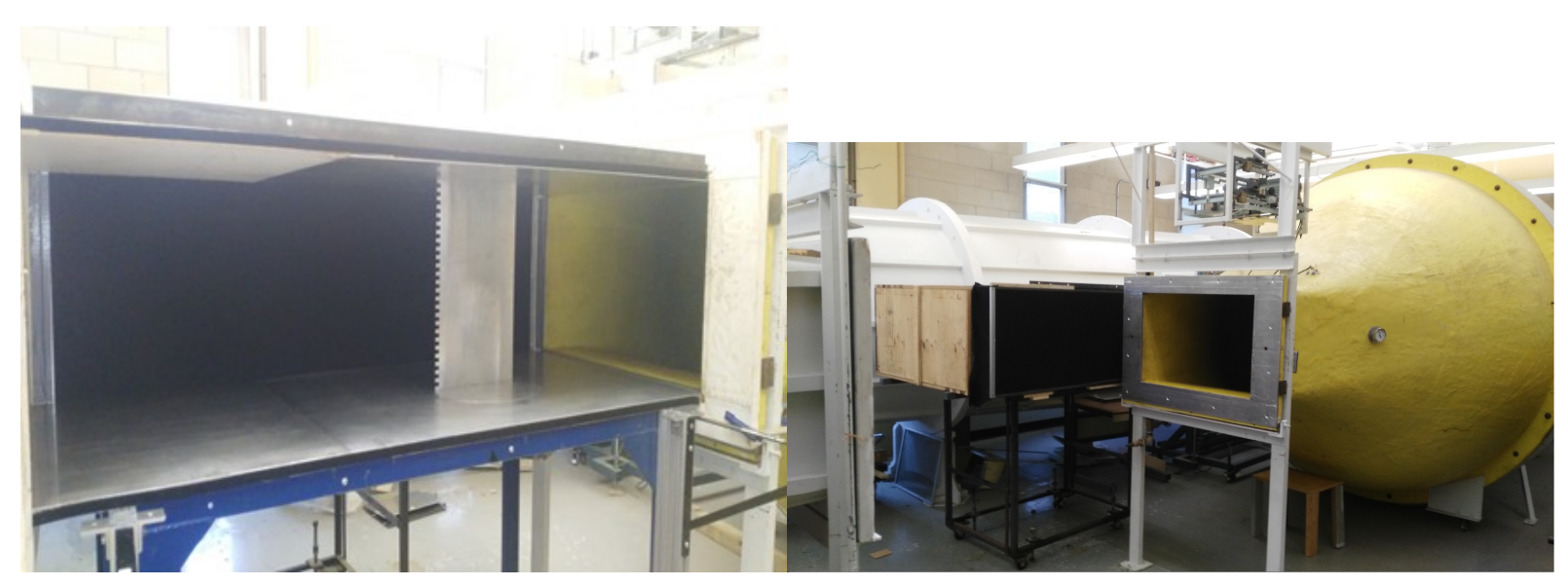

Figure 4.4: Wind tunnel at Carleton University.

\subsubsection{Flat-plate model with TE serrations}

The flat-plate model is composed of an aluminum body and a trailing-edge plate, as shown in Figure 4.5. The main body has a span of $510 \mathrm{~mm}$ and a thickness of $3 \mathrm{~mm}$. Five TE plates were fabricated and tested one by one: one straight, unserrated configuration and four with sawtooth serrations. The flat-plate model with the straight, unserrated trailing edge is used as the reference configuration for all tests and will hereafter be referred to as the reference plate. The four sawtooth configurations from the optimization study presented in Table 4.3 were used in the experimental validation to allow for direct comparison. The parameters $2 h$ and $\lambda$ are defined in Figure 4.1. Figure 4.5(b) shows the trailing-edge plates and the flat-plate model with TE sawtooth serrations attached to the wind tunnel contraction. The area of the reference plate is equivalent to that of the flat-plate with serrated trailing-edges giving the same effective wetted surface area in all four test cases. The serrated and reference plate models all have the same mean chord of $\mathrm{c}=300 \mathrm{~mm}$. The flat-plate model was then held between two side plates and extended beyond the width of the contraction outlet at zero angle-of-attack, AoA, as shown in 
Figure 4.5(a).

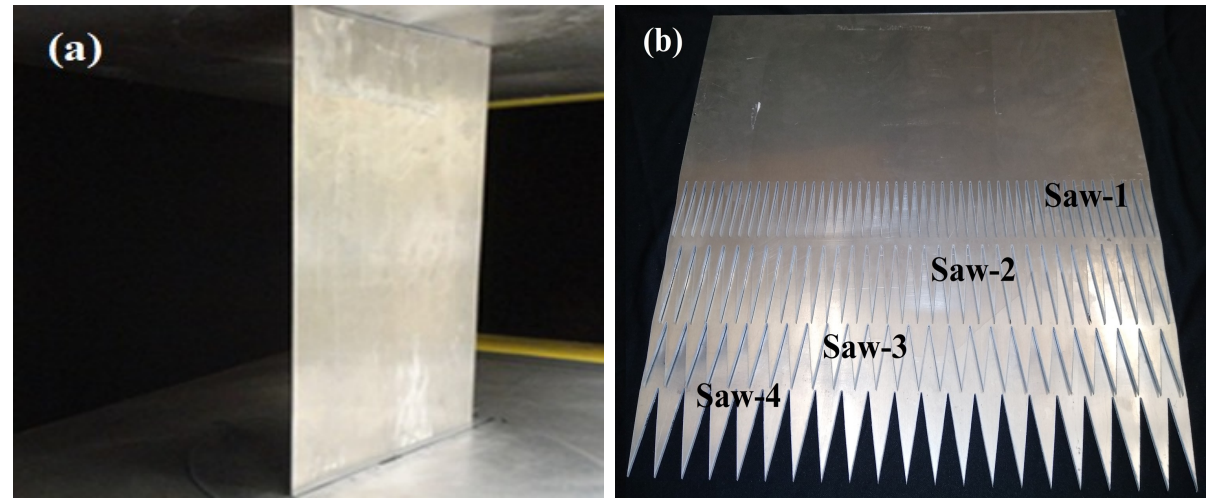

Figure 4.5: Flat-plate model with TE serrations.

\subsubsection{Airfoil model with TE serrations}

The airfoil under investigation is a NACA-0012 airfoil with sawtooth TE serrations cut directly into the main body of the airfoil, as shown in Figure 4.6. The parameters (similar sawtooth serration parameters as the flat-plate case above) associated with an airfoil serrated trailing-edge geometry include the serration amplitude $2 h$, and serration wavelength $\lambda$. The airfoil model with the straight, unserrated trailing-edge is used as the reference configuration for all tests and so will be referred to as the reference. The chord length of the airfoil is $300 \mathrm{~mm}$, and the span is $510 \mathrm{~mm}$. Between the leading-edge $\mathrm{x} / \mathrm{c}=0$ and $\mathrm{x} / \mathrm{c}=0.73$, the original NACA-0012 airfoil profile is unmodified, where $\mathrm{x}$ is the streamwise direction. Further downstream, $0.73 \leq \mathrm{c} \leq 1.0$ or $\left[T E_{\max }=80 \mathrm{~mm}\right]$, can be removed and replaced by either an unmodified or modified trailing-edge profile. Once attached, the trailing-edge section forms a continuous profile giving the appearance that the sawtooth serrations are cut into the main body of the NACA-0012 airfoil. 

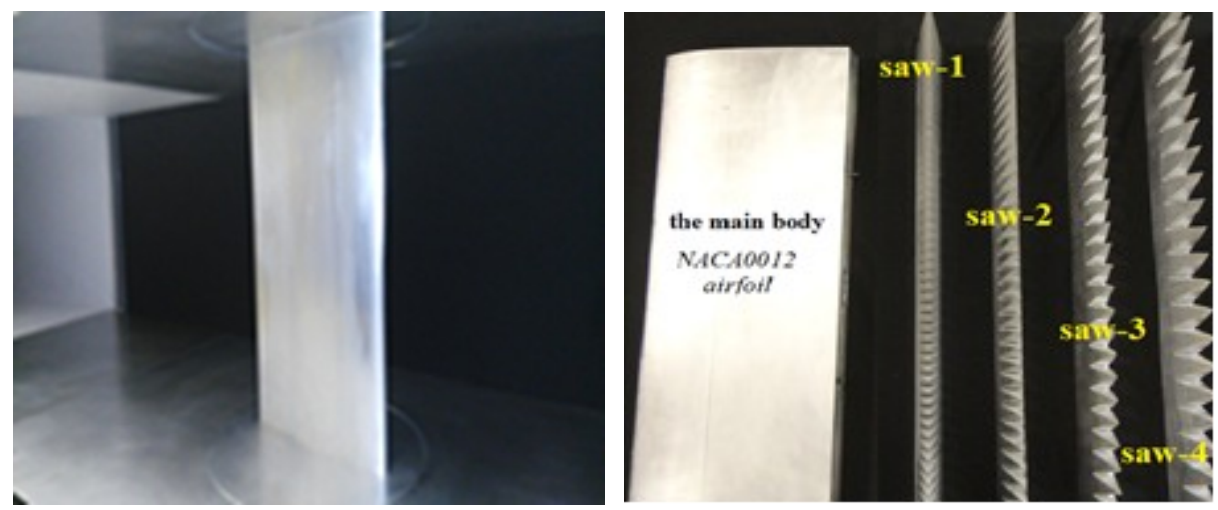

Figure 4.6: 2-D airfoil model with TE serrations.

\subsubsection{Instruments and procedure}

\subsubsection{Data Acquisition System}

A NI PXIe-1071 DAQ system was used for data acquisition. The system (shown in Figure 4.7) is composed of a 24-bit, eight channel National Instruments PXIe-4492 Sound and Vibration module housed in and powered by a PXIe-1071 DAQ chassis with a PXIe-8381 Remote Control Module. The chassis and module are designed to supply an excitation current to the amplifiers within the microphones through AO 0587-D-100 shielded cables. The data is recorded on a desktop computer using software written in LabVIEW. 


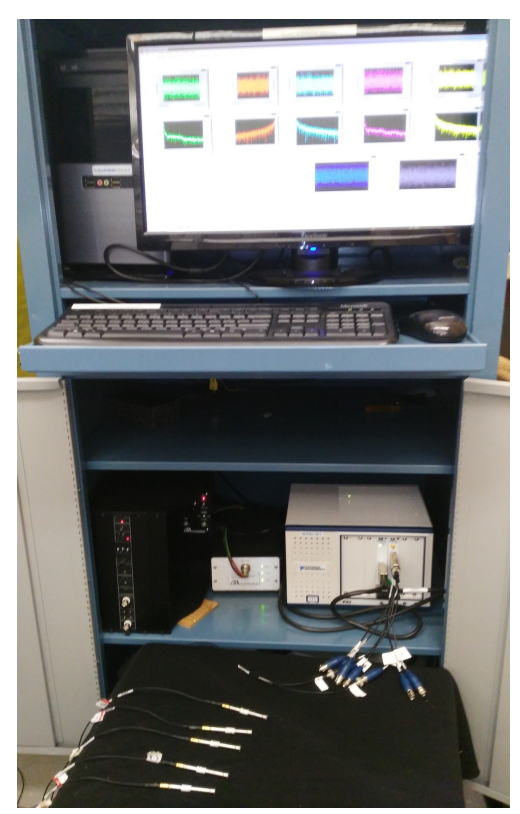

Figure 4.7: National Instruments PXIe-1071 DAQ system.

\subsubsection{Microphone Measurement System}

Measurements were made to determine the acoustic performance of the model using highperformance Bruel \& Kjaer (B\&K) 4944-A, $\frac{1}{4}$ inch (3.35mm) pressure field microphones with built-in DeltaTron preamplifiers. The microphones are fitted with grid caps (Figure 4.8 over the sensor diaphragm for free-field acoustic measurements, and to minimize the self-noise of the microphone. These microphones have a flat frequency response from roughly 50-10,000 Hz with a typical response curve shown in Figure 4.8. The microphones have a maximum sound pressure limit of $182 \mathrm{~dB}$ and are always connected to the data acquisition system with their respective AO 0587-D-100 Connection wires. 

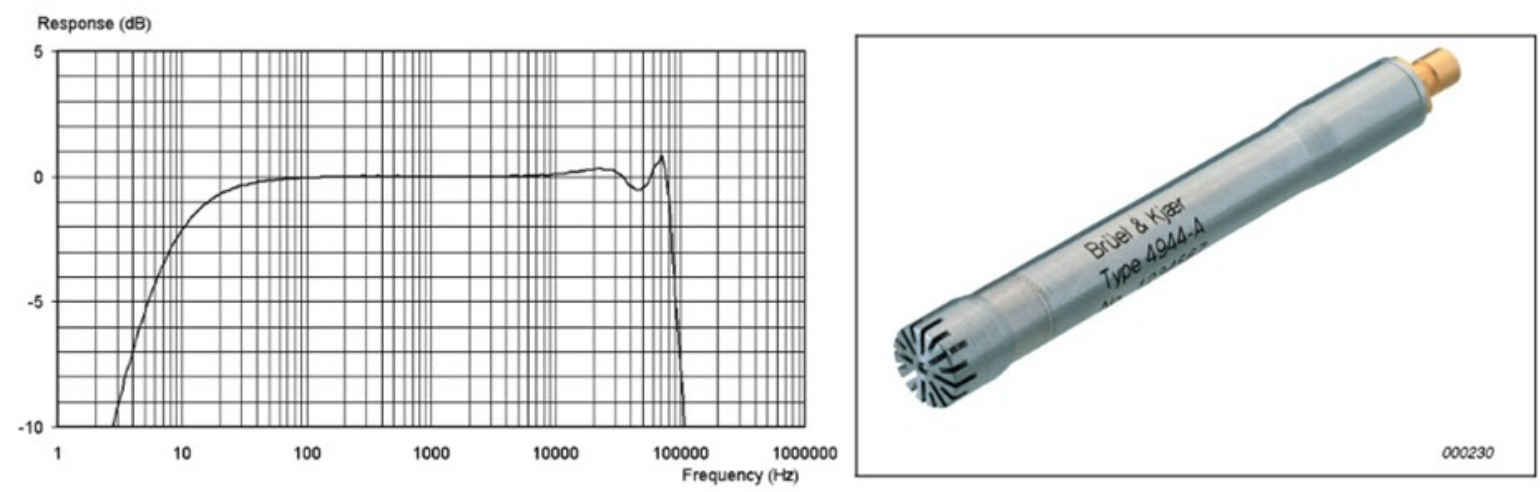

Figure 4.8: Typical 4944-A microphone and its frequency response.

\subsubsection{Microphone calibration}

A Svantek model SV 34 (114 dB tone at $1000 \mathrm{~Hz}$ ) sound pressure level meter calibration (shown in Figure 4.9), was used to verify the calibration of the microphones. Figure 4.10 shows the SPL plot for microphone 1 as measured at a sampling rate of $10 \mathrm{kHz}$. The resulting output is as expected with a SPL at $1000 \mathrm{~Hz}$. The other microphones performed similarly; thus, the default calibrations are used for the remainder of the work based on these results. The results from microphone 1 is presented in Figure 4.10 .
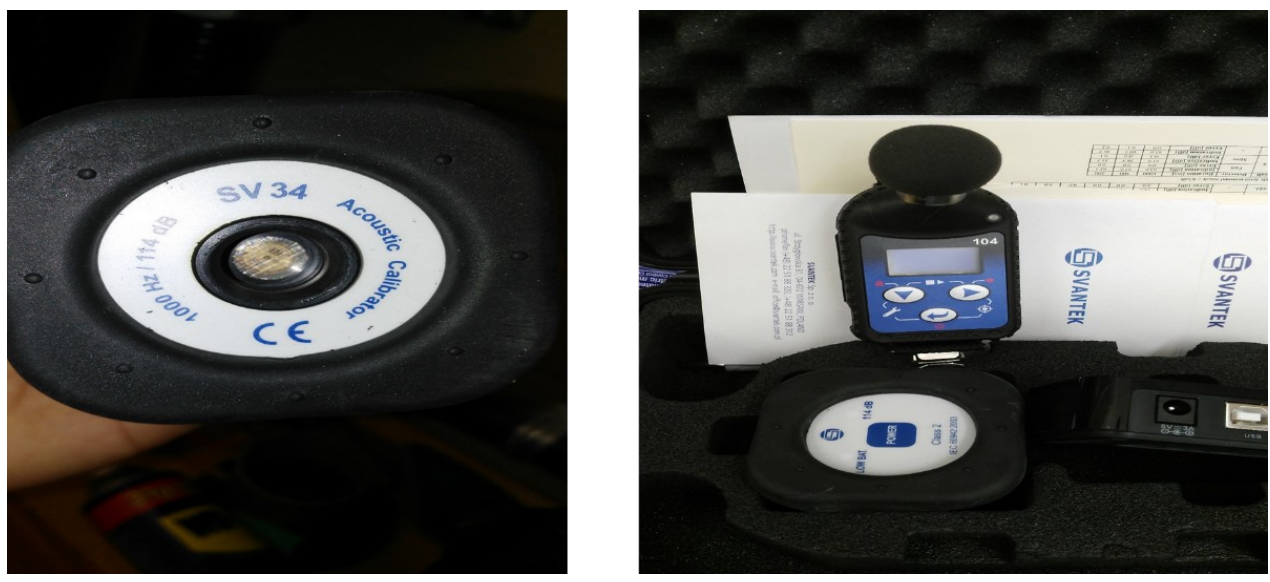

Figure 4.9: Left: Acoustic calibrator. Right: sound pressure level meter. 


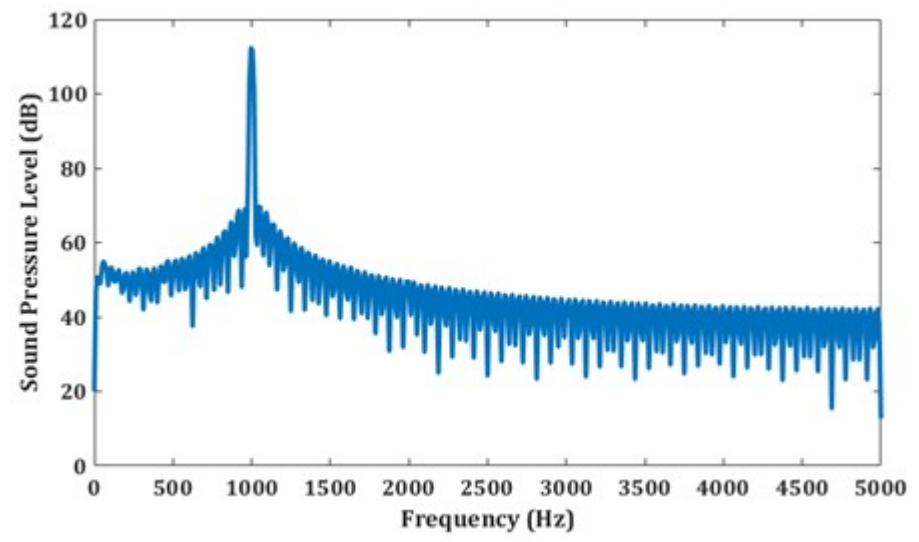

Figure 4.10: The calibration result obtained for microphone 1.

\subsubsection{Data analysis procedure}

To measure the radiated self-noise from the airfoil, a single calibrated microphone (Bruel \& Kjaer (B\&K) 4944-A, $\frac{1}{4}$ inch) is mounted at an angle of $\theta=90^{\circ}$, and distance of 1.4 m (as shown in Figure 4.11) perpendicular to the airfoil trailing-edge at mid-span. The microphone recorded the pressure fluctuation signal with respect to time. This data is used to obtain the power spectral density, PSD. Signals from the microphone were amplified by a B\&K Nexus amplifier before digitally stored in a computer through an $\mathrm{A} / \mathrm{D}$ converter of 24-bit resolution. During the experiment, the acoustic data is sampled at $20 \mathrm{kHz}$ and recorded for 30 seconds. The digitized data was passed through a time-domain filter to remove low and high frequency contamination caused by the microphone's low frequency roll-off and high-frequency aliasing. The band-pass filter used is a Butterworth filter with the first and second stop-band frequencies of 100 and fs $/ 2 \mathrm{~Hz}$, respectively, where fs is the sampling frequency. The attenuation is $60 \mathrm{~dB}$ for both the first and second stop-band. The PSD is then computed using the root mean square (rms) of filtered pressure using the following equation: 


$$
P S D(f)=10 \log _{10}\left(\frac{\left(P_{r m s}^{2} / H z\right)}{P_{r e f}^{2}}\right)
$$

where $P_{r e f}$ is the standard reference pressure in air, $20 \mu P a$.

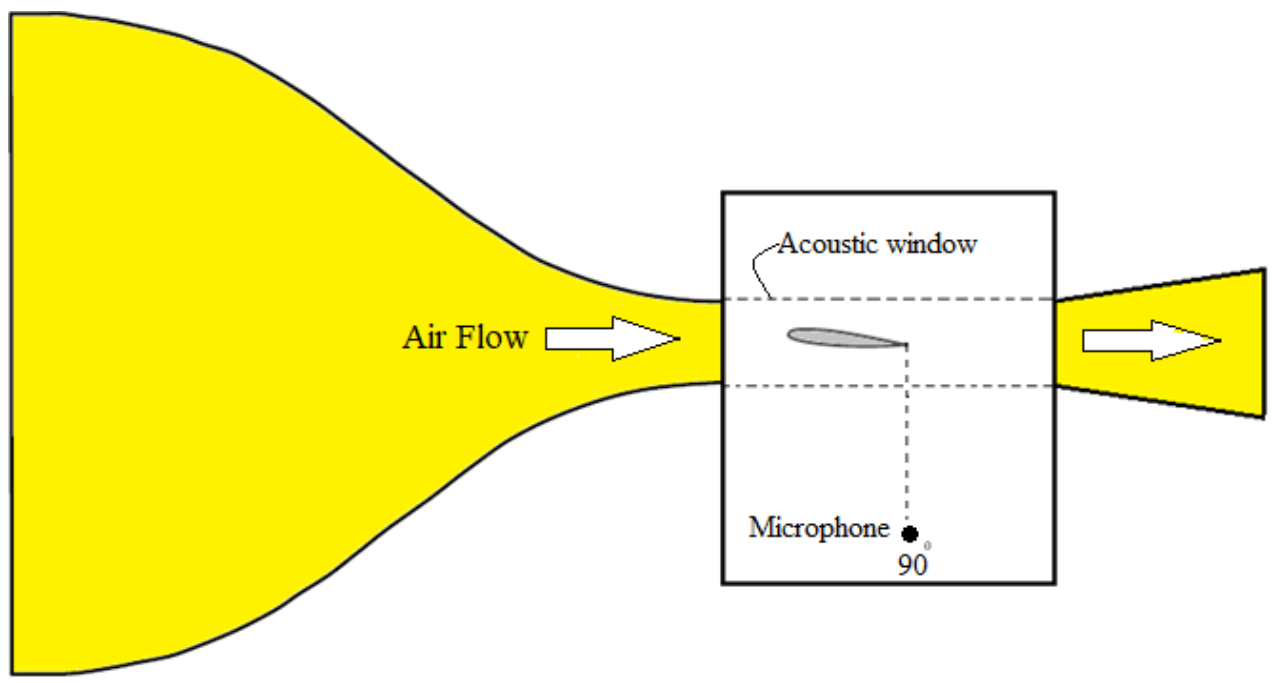

Figure 4.11: Experimental setup for the aeroacoustic test of the airfoil noise (top view).

\subsection{Comparison of experimental data with optimiza- tion results}

To evaluate the semi-empirical model performance and its discrepancy with the real case, the straight trailing-edge configuration for a parallel gust $\left(k_{3}=0\right)$ is computed for a flat-plat. The results are shown in Figure 4.12 and compared with the far-field noise 
measurements obtained in the wind tunnel at Carleton University. The power spectral density level was determined from sound measurements at $90^{\circ}$ above the trailing-edge, at the mid-span position at $1.4 \mathrm{~m}$ from the trailing-edge. Details are provided in Table 4.2 . The difference between the model results and the measurements is around $\sim(0$ to 4$) \mathrm{dB}$. It is then concluded that the model predicts the noise level accurately, and may serve to compute the relative noise reduction compared to the straight trailing-edge. The spectra obtained for this prediction for the airfoil and flat-plate are used as a reference value to compute the overall noise reduction from equation 4.16 .
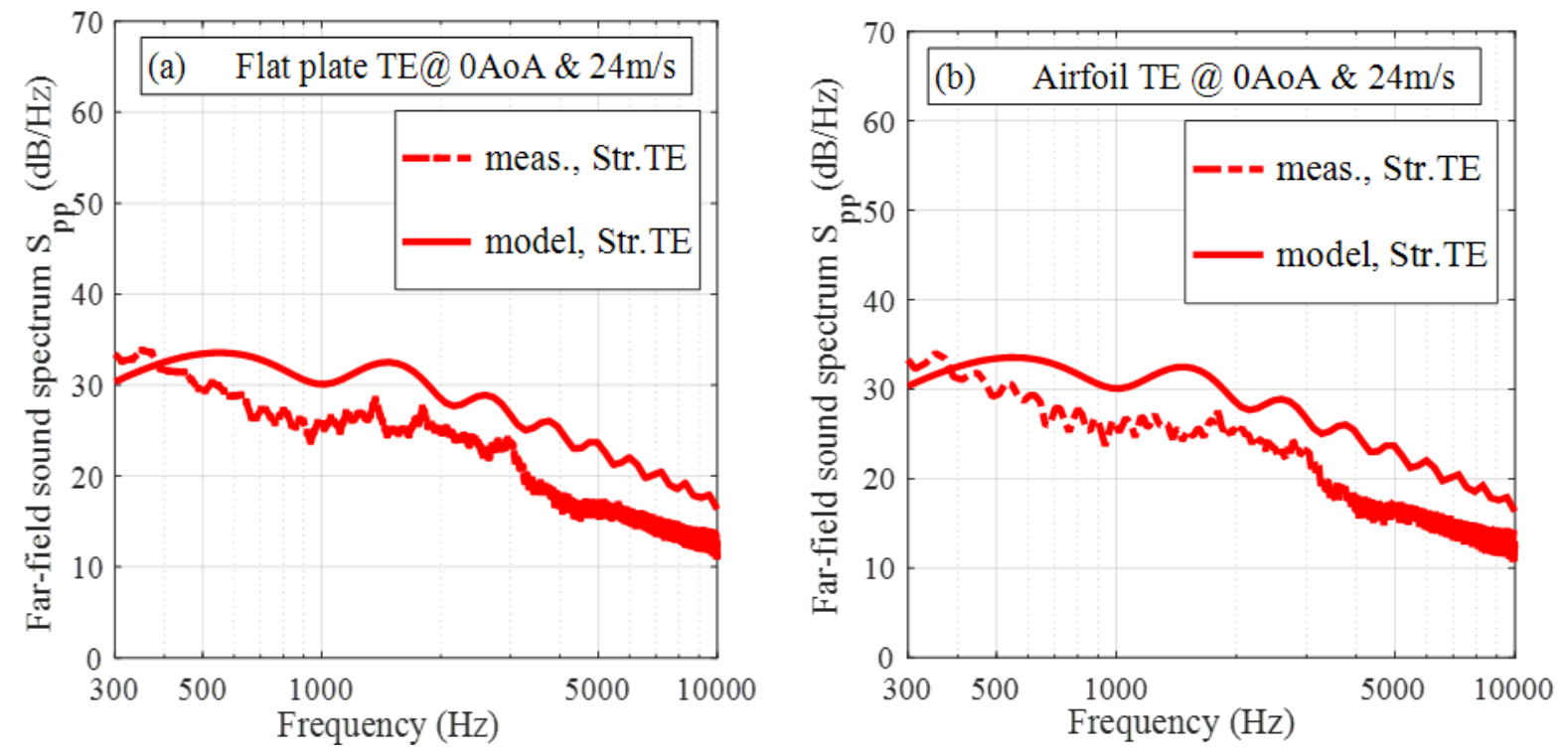

Figure 4.12: Power spectrum density level at mid-span and $90^{\circ}$ above the trailingedge of the: (a) flat-plate (b) NACA-0012 airfoil, computed with Lyu's model and compared with the experimental measurements.

To validate the optimization results, the far-field noise predictions were compared against the experimental data discussed in this section. The experiment was carried out for an NACA-0012 airfoil and a flat-plate immersed in a turbulent flow with a mean flow velocity of $\mathrm{U}=24 \mathrm{~m} / \mathrm{s}$. Figures 4.13 and 4.14 compare the measured far-field PSD 
spectrum of the flat-plate and airfoil trailing-edges, respectively, with the values predicted from the optimization in section 4.4. The high level of noise at low frequencies observed in the experimental data, as mentioned by Narayanan et al. [91], is believed to be due to the dominance of the wind-tunnel jet. Therefore, a direct comparison is not usually performed for frequencies less than $300 \mathrm{~Hz}$. Across the entire frequency range, an airfoil TE noise reduction of $\sim(1$ to 4$) \mathrm{dB}$ can be seen for all sawtooth trailing-edge configurations relative to the straight trailing edge, for the experimental results. Both airfoil and flat-plate have a similar trend and show that Saw-1 causes greatest measured noise reduction.

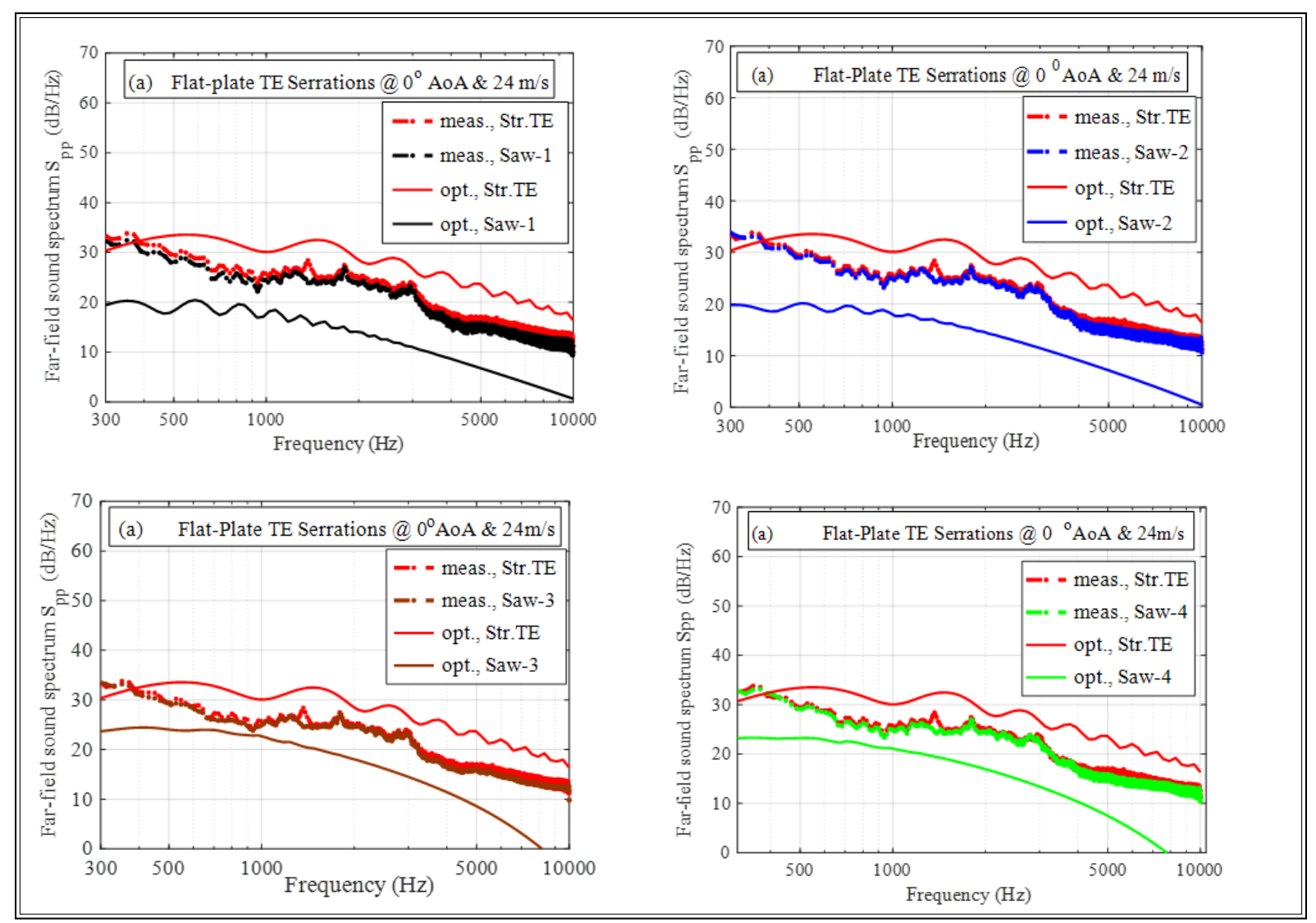

Figure 4.13: Comparison of the noise spectra between optimization predictions and experimental measurements at $24 \mathrm{~m} / \mathrm{s}$ and $0^{0}$ AoA for flat-plate sawtooth TE serrations 


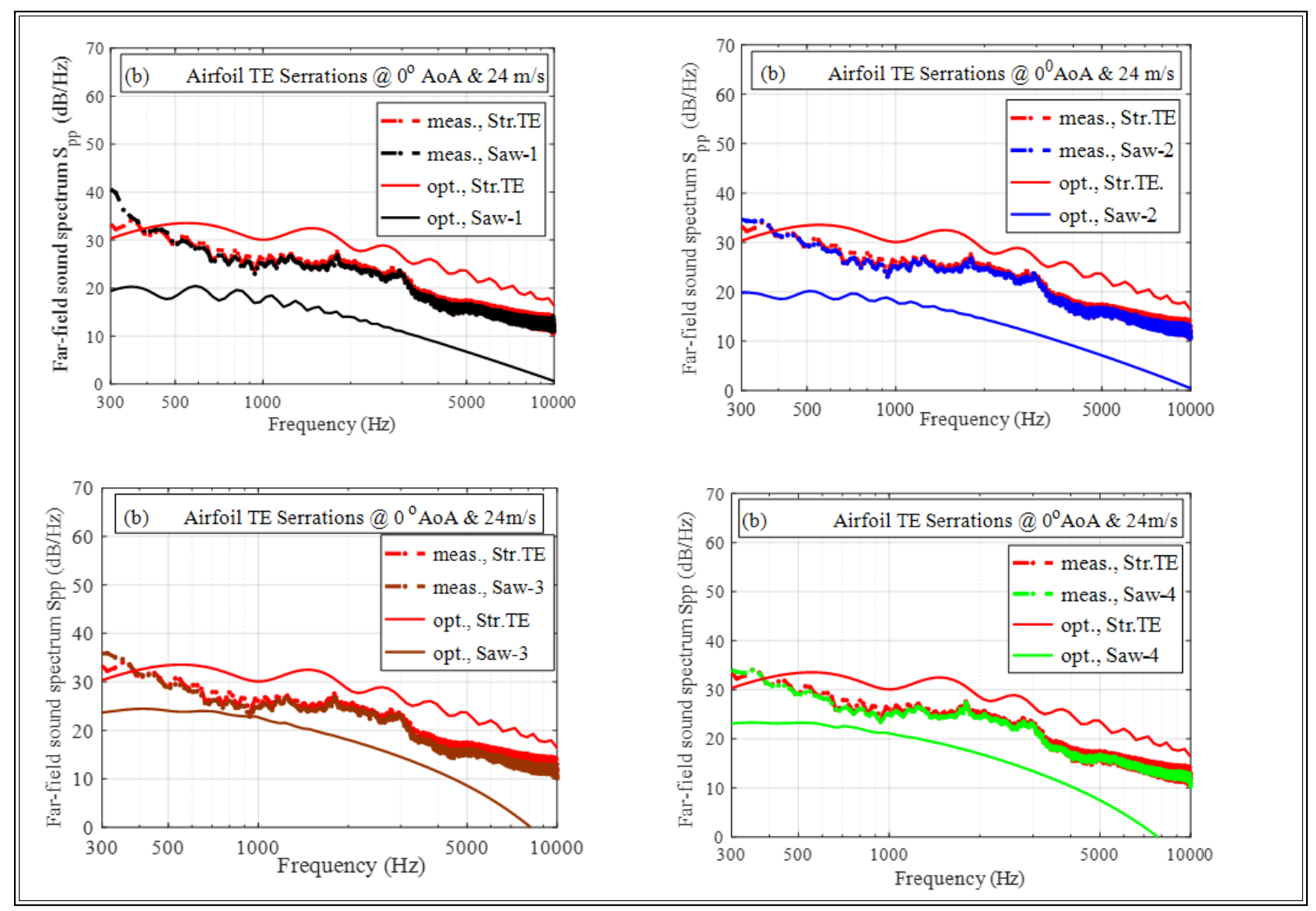

Figure 4.14: Comparison of the noise spectra between optimization predictions and experimental measurements at $24 \mathrm{~m} / \mathrm{s}$ and $0^{0}$ AoA for NACA-0012 airfoil sawtooth TE serrations.

Results from the optimization are directly comparable with experimental data. The far-field sound spectrum measured with a microphone was compared to the sound spectrum level predicted by the presented numerical optimization for a Reynolds number of $5 \times 10^{5}(24 \mathrm{~m} / \mathrm{s})$ and $0^{0}$ AoA in Figures 4.13 and 4.14 , both the serrated (sawtooth) and straight $(\mathrm{h}=0)$, spectra are shown. The numerical optimization underestimated the measurements by about $\sim(2$ to 12$) \mathrm{dB}$, but the tendency between the straight $\mathrm{TE}$ and the different sawtooth serrations was predicted correctly. i.e., the optimization results predicted that Saw-1 is quieter than Saw-2, Saw-3, and Saw-4, this was also validated by the experimental results. Both the straight and the sawtooth serration models predicted the wavy pattern of the noise spectrum level as a function of the frequency; however, the measurements were scattered to contradict this wavy pattern. 
The discrepancies observed in Figures 4.13 and 4.14 between the optimization and measured noise reductions for sawtooth trailing-edges indicate limitations of adopting the frozen turbulence assumption. A modified model that includes the growth/decay in the turbulent wall pressure fluctuation along the serration would likely show a better qualitative agreement with the experimental observations. One concludes that there is still room to improve the accuracy of the noise prediction model for trailing-edge serrations. A way to improve the model, and therefore optimization results, could be to include the effects of boundary layers development along the serrations. Nevertheless, the results are promising and the current study was successful in identifying trailing-edge serration designs that reduce airfoil noise.

\subsection{Conclusion}

Noise radiation from optimized trailing-edges with different sawtooth serrations have been investigated. It has been shown that the sawtooth TE serration design can have a significant effect on the level of noise reduction. Four different sawtooth designs of trailingedge serrations have been optimized for the reduction of TBL-TE noise. The optimum size of sawtooth tends to maximum amplitude and minimum wavelength. Moreover, based on the optimization results, the Saw-1 serration provides the greatest noise reduction of up to $12.89 \mathrm{~dB}$ over a wider range of frequencies. It is concluded that numerical and experimental results agree that optimized serrated trailing-edge configurations can yield less TBL-TE noise compared to the traditional straight-trailing-edge configuration. The optimization studies underestimated the measurements by about $\sim(2$ to 12$) \mathrm{dB}$, but the tendency between the different serration geometries was predicted correctly.

The optimization problem solved in this study is scalable for various types of constraints on the design space. It can be successfully implemented to solve a multi-objective 
optimization problem which includes aerodynamic and/or structural objectives. Also, the trends predicted in the numerical optimization section are well supported by the experiment. The current study focuses on sawtooth trailing-edge noise and its reduction mechanism is known to be similar to that for the turbulent boundary layer leading-edge noise. Hence, the present numerical optimization analysis and the optimum proposed sawtooth profile are expected to work in a similar manner for the leading-edge problem. 
Chapter 5

\title{
ELES and Tonal Noise Prediction of
}

\section{Serrated-TE Airfoils}

\begin{abstract}
In this work, Embedded Large Eddy (ELES) Simulations are employed in tandem with the Ffowcs Williams-Hakings (FW-H) model to predict the tonal peaks of NACA0012 airfoils having different noise-suppressing trailing-edge (TE) configurations. Different nonflat plate sawtooth serration configurations are investigated and experimental wind tunnel testing is performed. ELES results are validated then compared with experimental measurements, and qualitative agreement is obtained in terms of flow statistics and the far-field noise spectra. TE sawtooth serrations are shown to significantly modify the aerodynamics of the wake and improve mixing across the airfoil. Experimental results confirm that sawtooth serrations reduce the broadband noise radiated by the airfoil at the expense of generating a tonal peak, caused by vortex shedding associated with the bluntness of the serration roots. Longer serrations, and higher values of root bluntness are responsible for the strength of the shed vortices and the intensity of the radiated tonal noise. The frequency at which the tonal peaks occur can be controlled for the same serration ampli-
\end{abstract}


tude and root bluntness by modifying the wavelength. Larger wavelength values for the same serration amplitude and root bluntness lead to higher tonal peak frequencies, and larger values of root bluntness for the same serration amplitude and wavelength lead to lower tonal peak frequencies accompanied by higher peak amplitudes.

\subsection{Introduction}

With the world growing increasingly noisier, governments and organizations are applying restrictions and regulations aiming to protect the planet and reduce the environmental footprint of our species. Therefore, aerodynamic noise reduction has been steadily gaining attention. Over the past century, especially over the past few decades, noise w pollution has escalated tremendously, disturbing the integrity of natural ecosystems and putting them at risk [92]. Even humans are suffering from noise pollution as it impacts their quality of life and puts their mental and physical well-being at risk 93 .

In parallel, global warming has led a universal push towards sustainability, promoting an increased interest in renewable power sources to replace coal and fossil fuels, and reduce greenhouse gas emissions. One of these sustainable resources is to harness the energy of wind through wind turbines. Despite their many advantages, the noise produced by such turbines is still of the most significant hindrance preventing their widespread use, and the largest contributor to this noise pollution is that generated by the trailing edge of wind turbine blades $|94|$. For those reasons, TE noise reduction has become a crucial challenge in many industrial sectors.

To investigate the possibility of having low-noise airfoils, engineers turned to nature, and in 1934, R. R. Graham $[95 \mid$ was the first to recognize the potential of using birds as a reference to render modern airplanes more efficient, and specifically identified owls as a biomimicry candidate to achieve silent flight. The wings of owls differ greatly from those 
of all other groups of birds. Three main noise reduction peculiarities were observed in owl wings, which distinguish them from other birds:

i. The Leading-Edge Comb: A remarkably stiff comb-like fringe exists on the front margin of every feather.

ii. The Trailing-Edge Fringe: A fringe resembling that of a shawl spans along the TE of the main wing.

iii. The Downy Upper Surface: Certain parts of the upper surface are covered with a short, fine down.

Soon afterwards, more engineers followed in the same path and were drawn to nature looking for inspiration. In 1998, Lilley $|96|$ confirmed the three main noise suppression mechanisms in owl wings previously addressed by Graham [95]. The author then discussed the aerodynamic characteristics of each of these devices and proposed explanations as to how the flow dynamics vary, leading to large noise reduction in the range of frequencies above $2 \mathrm{kHz}$. According to him:

i. The comb-shaped leading-edge feathers behave as closely spaced co-rotating vortex generators creating streamwise vortices which lead to a reduction in BL thickness and in the volume of turbulence crossing the TE.

ii. The trailing-edge fringe resembles a serrated edge which suggests the reduction or even elimination of TE scattering.

iii. Given the small diameter of its fibers, the compliant velvety surface of the wing acts as a bypass mechanism for energy dissipation at frequencies smaller than the conventional dissipation range of frequencies associated with viscous damping. In 
other words, the fibers absorb energy from the small-scale noise-emitting eddies, thus silencing them.

Following the work of these authors, even more researchers took interest in using the owl as a biomimicry model. The proceeding step was to implement owl wing features in a practical way on solid airfoils to study their efficiency as noise suppressers and their effect on aerodynamic flow properties. Extensive experimental work has been conducted to confirm the applicability of TE extensions, serrations in particular, as noise suppression devices $58,76,97,98$. Chong et al. 97] experimentally investigated the feasibility of employing different serrated TE configurations to reduce the noise produced by a NACA0012 airfoil. For the case of an untripped boundary layer, testing velocities of up to $60 \mathrm{~m} / \mathrm{s}$ and an angle of attack of $4.2^{\circ}$, it was shown that sound power reduction of up to $30 \mathrm{~dB}$ is possible for the instability tonal noise. When the airfoil surface is tripped, broadband sound power reduction of $6.5 \mathrm{~dB}$ was achieved. However, more significant narrowband noise is generated by the vortex shedding at the serration roots. The authors concluded even though smaller serration angles lead to better broadband noise reduction, larger angles are recommended to account for the unavoidable narrowband vortex shedding noise.

Numerical simulations were used to predict far-field noise radiation. In 2000, Manoha, Troff and Sagaut $|99|$ successfully predicted the far-field noise generated by turbulence flowing over the blunt $\mathrm{TE}$ of a thick flat plate by coupling a Large Eddy Simulation (LES) with Curle's solution to the Lighthill equation, then the Ffowcs-Williams and Hawkings (FW-H) analogy. Agrawal et and Sharma $|100|$ also assessed the effectiveness of biomimicry in reducing aerodynamic noise using LES. The interaction between the wake of a cylindrical rod and a downstream airfoil was simulated to investigate the effect of sinusoidal leading-edge serrations on radiated noise. Tang et al. 101 performed simulations employing LES using the Lighthill-Curle method in an attempt to reveal the variation in the hydrodynamic field and sound source associated with TE serrations on a 
NACA 0012 airfoil. It was confirmed that TE serrations reduce the radiated noise seeing that serrations impede the growth of spanwise vortices, i.e. decrease spanwise coherence, and promote streamwise ones near the wake. Zilstra and Johnson [102] demonstrated the ability of LES, combined with the FW-H acoustic analogy, to predict the flow field and acoustic results for a SD7037 airfoil at a Re of 43,000 and different angles of attack. Overall, the method proved to be an effective airfoil self-noise prediction tool at static angles of attack $(A O A s)$.

Despite the increase in computing power over the last two decades, Large Eddy Simulations remain prohibitively expensive. Because of the impracticality of LES and the need for reliable short-response-time noise prediction methods for industrial design and optimization, some researchers resorted to statistical models based on steady RANS solutions in a sequential CFD/CAA approach. Rumpfkei [103] reviewed three different methods based on steady Reynolds-averaged Navier-Stokes (RANS) solutions to predict noise emitted from airfoils [104-106]. Validation studies showed decent agreement between the considered methods and results from experiments, a semi-empirical airfoil self-noise prediction code and LES. In another attempt to get accurate noise predictions at a reasonable computational cost, Quemere and Sagaut [107] presented a novel zonal multi-domain RANS/LES method (also known as Embedded LES or ELES), where the full domain configuration was decomposed into several subdomains that can be treated with either RANS or LES. The same concept was later adopted by Teraccol [108], who investigated using ELES to represent aerodynamic noise sources. The method was applied to a flat plate with a blunt TE and a NACA-0012 airfoil. In this approach, zonal LES is only performed close to the main elements responsible for sound generation, while the overall configuration is treated by a RANS. The most critical point was the numerical treatment performed at the inlet of the LES domain. CPU time reductions in the order of 40 were obtained and the method was found to be an attractive compromise between accuracy and computational cost. In 2008, Frohlich and von Terzi [109] presented a generic review 
of the various ELES approaches along with different interface treatment strategies. The review provided information on how to distinguish between the different methods and to further the understanding of their inherent limitations as well as the encountered difficulties. Successful simulation results demonstrated the high potential of the approach. In the same year, Mathey [110 evaluated using the ELES approach for the prediction of broadband and tonal noise generated by the flow past an airfoil TE at a high Re. Two simulations were performed for a free stream velocity of $30.5 \mathrm{~m} / \mathrm{s}$ and a chord based Re of $1,800,000$. The first one used a random forcing method at the RANS/LES interface, and the second one used the Vortex Method. The far-field noise was calculated using the FW-H model. The results showed that the technique is capable of capturing the separated flow and reproducing the main characteristics of the aeroacoustic sources. Lastly, it was shown that the use of the Vortex Method (VM) for the generation of a synthetically turbulent flow field significantly improved the accuracy of the simulation. Kim et at. 66 used a segregated ELES approach to predict the aeroacoustic and aerodynamic properties of several flatback airfoils at high $R e$ and compared the results to semi-empirical and experimental data. Synthetic turbulence was generated at the RANS/LES interface using the Vortex Method and far-field acoustics were computed using the FW-H analogy. The obtained frequency spectra of surface pressure fluctuations obtained is in good agreement with experimental measurements at the same observer location and the hybrid RANS-LES method is found to be adequate for predicting aerodynamic noise generation by vortical flow in the vicinity of a blunt TE airfoil over a range of frequencies. Lane, Croaker and Ding [111 tested and implemented ELES for the prediction of TE noise due to flow around a NACA-0012 airfoil. The obtained results were compared to a full LES simulation and to experimental data. Both simulations used the same mesh resolution and the same wall-modeled LES approach. For ELES, the mesh size was only about 13 million cells, compared to 40 million cells for the full LES. It was found that the results of both simulations were in good agreement. The ELES approach resulted in an impres- 
sive saving of $55 \%$ of the computational cost of a full LES. Zuo et al. [112 performed flow simulations using ELES to analyze the aerodynamic and noise characteristics of a serrated-TE NACA-0018 airfoil at a Re of 160,00 and an angle of attack of 6 degrees. Two airfoils having the same serration wave length and different serration amplitudes were considered and compared to a plain straight TE case. Predictions based on the FW-H acoustic analogy showed that longer serrations are more effective in decreasing the overall sound pressure levels.

In the present work, ELES is adopted to study the flow field around a flat-TE NACA0012 airfoil as well as three serrated-TE airfoils having different serration amplitudes and wavelengths, at zero AoA. The far-field noise is computed using the Ffowcs Williams-Hawkings (FW-H) model and attention is given to the tonal peaks generated by the serrated airfoils. The flow chord-based Reynold's number, $R e_{c}$, is approximately 500,000. The computational results are validated and compared with available experimental data. The used ELES configuration, where the LES region only partially covers the airfoil chord-length, hasn't been used to investigate bio-inspired TE designs yet. In this context, the main goals of this study are to provide a faster alternative to the currently used computationally prohibitive simulation models and use it to visualize the flow field around TE serrations, as well as assess the effect of changing different serration parameters on the radiated tonal noise.

This chapter is structured as follows. Section 5.2 presents the numerical methodology with the governing LES equations (Section 5.2.1), Section 5.3 describes the hybrid RANS/LES interface treatment and Section 5.4 presents the FW-H aeroacoustic analogy. Thereafter, Section 5.5describes the flow configuration (Section 5.5.1), the computational mesh (Section 5.5.2) and the experimental setup (Section 5.5.3). All results are presented in Section 5.6 . 


\subsection{Numerical Methodology}

\subsubsection{Governing Equations}

The governing equations used in the current study, termed the spatially-filtered NavierStokes equations, are obtained by applying a low pass filter on the time dependent NavierStokes equations in the physical space. The flow is assumed incompressible. In order to increase efficiency, the filter width is the same size as the mesh spacing used in the computational domain. The resulting equations describe the dynamics of large eddies [113. Field variables, such as pressure and velocity, are defined by their convolution with a filter function over the fluid domain:

$$
\bar{\Phi}(x)=\int_{D} \phi\left(x^{\prime}\right) G\left(x, x^{\prime}\right) d x \prime
$$

where $\mathrm{D}$ is the fluid domain and $\mathrm{G}$ is the filtering function. The overbar indicates spatial filtering and not temporal averaging. After applying the filter to the mass and momentum conservation equations, the NS equations become:

$$
\begin{gathered}
\frac{\partial \rho}{\partial t}+\frac{\partial}{\partial x_{i}}\left(\rho \bar{u}_{i}\right)=0 \\
\frac{\partial}{\partial t}\left(\rho \bar{u}_{i}\right)+\frac{\partial}{\partial x_{j}}\left(\rho \bar{u}_{i} \bar{u}_{j}\right)=\frac{\partial}{\partial x_{j}}\left(\sigma_{i j}\right)-\frac{\partial \bar{p}}{\partial x_{i}}-\frac{\partial \tau_{i j}}{\partial x_{j}}
\end{gathered}
$$

In order to obtain a close system of equations, the unknown SGS stresses are modeled by applying the Boussinesq eddy viscosity hypothesis [114], thus computing the subgridscale turbulent stresses from

$$
\tau_{i j}-\frac{1}{3} \tau_{k k} \delta_{i j}=-2 \mu_{t} \overline{S_{i j}}
$$


where $\mu_{t}$ is the subgrid-scale turbulent viscosity and $\tau_{k k}$ is the isotropic part of the SGS. The latter part is not modeled as it is added to the filtered static pressure term. $S_{i j}$ is the strain-rate tensor of the resolved scale calculated from equation $(5.5)$ using the filtered velocity components.

$$
\bar{S}_{i j}=\frac{1}{2}\left(\frac{\partial \bar{u}_{i}}{x_{j}}+\frac{\partial \bar{u}_{j}}{\partial x i}\right)
$$

For the Wall Adapting Local Eddy Viscosity (WALE) model [115], $\mu_{t}$ is modeled as:

$$
\mu_{t}=\rho L_{s}^{2}\left(\frac{\left(S_{i j}^{d} S_{i j}^{d}\right)^{\frac{3}{2}}}{\left(\overline{S_{i j}} d \bar{S}_{i j}\right)^{\frac{5}{2}}\left(S_{i j}^{d} S_{i j}^{d}\right)^{\frac{5}{4}}}\right)
$$

$L_{s}$, the mixing length of the sub-grid scale, and $S_{i j}^{d}$, which is a function of the strain and rotation rate tensors, are defined in equations 5.7 and 5.8 as

$$
\begin{gathered}
L_{s}=\min \left(\kappa d, C_{w} V^{\frac{1}{3}}\right) \\
S_{i j}^{d}=\frac{1}{2}\left(\bar{g}_{i j}^{2}+\bar{g}_{j i}^{2}\right)-\frac{1}{3} \delta_{i j} \bar{g}_{k k}^{2}
\end{gathered}
$$

And $g_{i j}$ is defined in equation (5.9) as

$$
\bar{g}_{i j}=\frac{\partial \bar{u}_{i}}{\partial x_{j}}
$$

where $d$ is the distance to the closest wall, $V$ is the volume of the computational cell, $\kappa=0.41$ is the von Karman constant and $C_{w}=0.325$ is the WALE constant. 


\subsection{RANS/LES Interface}

In the present approach, the entire flow domain is decomposed into clearly identifiable regions for RANS and LES before the simulation is started. This is usually referred to as segregated modeling. The goal is to use each model where it is best suited. The flow is initialized using RANS equations, which provide stationary field statistics, and LES resolves the unsteady high-resolution perturbations near the TE, where it is needed. The main difficulty is defining proper interface conditions, seeing that inappropriate coupling could lead to results contamination in the LES or RANS subdomains.

At the inflow interface, mass, momentum and energy are convected into the LES subdomain from the RANS region. The latter provides mean values which are to be coupled with the LES data. To obtain correct LES results, fluctuations must be provided at the interface and added to the mean flow computed by RANS. These fluctuations can be real, provided by precursor simulations or databases of similar flows, or synthetic, provided by Fourier modes, digital filters, random vortices...etc. The goal is to make the imposed fluctuation as close as possible to those present in a real physical flow.

The Vortex Method [116 was chosen as a means of adding artificial resolved turbulence at the RANS/LES interface. In this approach, a fluctuating vorticity field is added to the mean flow, consequently creating perturbations similar in behavior to realistic ones. The VM is based on the Biot-Savart law and the $2 \mathrm{D}$ evolution equation of vorticity. Vortex points, or particles, are distributed over the inlet interface perpendicular to the streamwise direction and are randomly convected, carrying information about the vorticity field. The amount of vorticity carried by a given particle " $\mathrm{i}$ " is represented by the circulation $\Gamma$ according to equation 5.11, and the assumed spatial distribution is given by equation 5.12 , such that

$$
\omega(\vec{x}, t)=\sum_{i=1}^{N} \Gamma_{i}(t) \eta\left(\left|\vec{x}-\vec{x}_{i}\right|, t\right)
$$




$$
\begin{gathered}
\Gamma_{i}(x, y)=4 \sqrt{\left(\frac{\pi A k}{3 N(2 \operatorname{In}(3)-3 \operatorname{In}(2))}\right)} \\
\eta(\vec{x})=\frac{1}{2 \pi \sigma^{2}}\left(2 e^{\frac{-|x|^{2}}{2 \sigma^{2}}}-1\right) 2 e^{\frac{-|x|^{2}}{2 \sigma^{2}}}
\end{gathered}
$$

Where $N$ is the number of vortex points, $A$ is the inlet section area, $k$ is the turbulence kinetic energy and $\sigma$ controls the size of the vortex particles. The resulting discretization for the velocity field is given by

$$
\vec{u}(\vec{x})=\frac{1}{2 \pi} \sum_{i=1}^{N} \Gamma_{i} \frac{\left(\left(\overrightarrow{x_{i}}-\vec{x}\right) \times \vec{z}\right)\left(1-e^{\frac{\left|\vec{x}-\vec{x}^{\prime}\right|^{2}}{2 \sigma^{2}}}\right)\left(e^{\frac{\left|\vec{x}-\vec{x}^{\prime}\right|^{2}}{2 \sigma^{2}}}\right)}{\left|\vec{x}-\overrightarrow{x_{i}^{\prime}}\right|}
$$

Where $\vec{z}$ is a unit vector in the streamwise direction. The value of $\sigma$ is calculated from a known profile of mean turbulence kinetic energy and mean dissipation rate at the inlet, such that

$$
\sigma=\frac{c k^{3 / 2}}{2 \epsilon}
$$

where $\mathrm{c}=0.16$. The minimum value of $\sigma$ is determined by the local mesh size to ensure that the vortices will always belong to the resolved scale. Furthermore, the sign of the circulation of each vortex is randomly changed every characteristic time scale, which is the time needed for a 2D vortex to travel $\mathrm{n}$ times its mean characteristic $2 \mathrm{D}$ size in the boundary normal direction, where $\mathrm{n}$ is set to equal 100 from numerical testing. Finally, a rescaling model is used, and the velocity fluctuations are expressed as 


$$
u^{\prime *}=u_{i}^{\prime} \frac{\sqrt{<u_{i} u_{i}>}}{\sqrt{\frac{2}{3 k}}}
$$

Where $u^{\prime *}$ and $u_{i}^{\prime}$ are the scaled and unscaled velocity fluctuations, and $<u_{i} u_{i}>$ represents the normal statistic velocity fluctuations.

\subsection{The FW-H Acoustic Analogy}

To overcome the prohibitive cost of directly resolving the pressure fluctuations responsible for noise in the far-field, a method based on Lighthill's acoustic analogy [7] is used. In this approach, the nearfield flow is computed using the appropriate governing equations of ELES, and the far-field noise is predicted with the aid of an analytically derived integral solution to the wave equation. The acoustic analogy decouples sound generation from its propagation, thus allowing the separation of the flow solution from the acoustic analysis and the extraction of acoustic sources from the CFD domain.

The Ffowcs Williams and Hawkings (FW-H) formulation [117 adopts the most general form of Lighthill's acoustic analogy. The FW-H equation 117, 118 is nothing but an inhomogeneous wave equation derived by manipulating the continuity and Navier-Stokes equation. The FW-H equation can be expressed as

$$
\begin{aligned}
\frac{1}{a_{\infty}^{2}} \frac{\partial^{2} p^{\prime}}{\partial t^{2}}-\nabla^{2} p^{\prime} & =\frac{\partial}{\partial t}\left\{\left[\rho_{\infty} \nu_{n}+\rho\left(u_{n}-v_{n}\right)\right] \delta(f)\right\} \\
& -\frac{\partial}{\partial x_{i}}\left\{\left[P_{i j} n_{j}+\rho u_{i}\left(u_{n}-v_{n}\right)\right] \delta(f)\right\}+\frac{\partial^{2}}{\partial x_{i} \partial x_{j}}\left\{T_{i j} H(f)\right\}
\end{aligned}
$$




$$
T_{i j}=\rho u_{i} u_{j}+P_{i j}-a_{\infty}^{2}\left(\rho-\rho_{\infty}\right) \delta_{i j}
$$

where $p l=p-p_{0}$ is the sound pressure at the far-field, $u_{i}$ is the fluid velocity component in the $x_{i}$ direction, $u_{n}$ is the velocity component normal to the surface $f=0, v_{i}$ is the surface velocity component in the $x_{i}$ direction, $v_{n}$ is the surface velocity component normal to the surface, $\delta(f)$ is the Dirac delta function and $H(f)$ is the Heaviside function. The subscript " $\infty$ " denotes free-stream parameters. The $f=0$ surface is a mathematical surface representing the source surface. $n i$ is a unit vector normal pointing towards the exterior region of the source $(f>0), a_{0}$ is the speed of the sound at the far field and $T_{i j}$ is the Lighthill stress tensor defined in equation 5.17. $P_{i j}$ is the compressive stress tensor. The first term on the RHS of equation 5.16 represents the monopole or thickness source, modeling the sound generated by the displacement of a fluid as a body passes through it. The second term is the dipole or loading source, resulting from the unsteadiness of the forces acting on the body's surface. The third term is the quadrupole source term, representing the non-linear fluctuations in the local sound speed and fluid velocity near the body surface. Monopole and dipole sources are dominant in low Mach number flows. By integrating equation 5.16 assuming free-space flow and no obstacles between the sound source and receiver, a full solution consisting of surface and volume integrals is obtained. In the present case, the volume integral is neglected as it is only significant in high Mach number flows. Thus, the far-field sound pressure can be expressed as

$$
p^{\prime}(\vec{x}, t)=p_{T}^{\prime}(\vec{x}, t)+p_{L}^{\prime}(\vec{x}, t)
$$

where 


$$
\begin{gathered}
4 \pi p_{T}^{\prime}(\vec{x}, t)=\int_{f=0}\left[\frac{\rho_{0}\left(\dot{U}_{n} U_{\dot{n}}\right)}{r\left(1-M_{r}\right)^{2}}\right] d s+\int_{f=0}\left[\frac{\rho_{0} U_{n}\left\{r M_{r}+a_{0}\left(\dot{M}_{r}-M^{2}\right)\right\}}{r^{2}\left(1-M_{r}\right)^{3}}\right] d S \\
4 \pi p_{L}^{\prime}(\vec{x}, t)=\frac{1}{a_{0}} \int_{f=0}\left[\frac{L_{r}}{r\left(1-M_{r}\right)^{2}}\right] d S+\int_{f=0}\left[\frac{L_{r}-L_{M}}{r^{2}\left(1-M_{r}\right)^{2}}\right] \\
d S+\frac{1}{a_{0}} \int_{f=0}\left[\frac{L r\left\{r \dot{M}_{r}+a_{0}\left(\dot{M}_{r}-M^{2}\right)\right\}}{r^{2}\left(1-M_{r}\right)^{3}}\right] d S \\
U_{i}=v_{i}+\frac{\rho}{\rho_{0}}\left(u_{i}-v_{i}\right) \\
L_{i}=P_{i j} \hat{n_{j}}+\rho u_{i}\left(u_{n}-v_{n}\right)
\end{gathered}
$$

A dot over a variable indicates the source-time derivative of that variable, while the subscripts "n", "r" and "M" denote the dot product with the unit normal vector, the unit radiation vector and surface velocity vector normalized by the speed of sound, respectively.

\subsection{Flow Configuration and Computational Setup}

\subsubsection{Flow Configuration}

The airfoil selected for the present study is a NACA-0012 symmetric airfoil to isolate the effect of lift generation on the radiated noise. The chord length of the airfoils $\mathrm{c}$ is 
$0.3 \mathrm{~m}$. The airfoil is placed in a square $10 c \times 10 c$ domain. The flow domain is divided into two regions as seen in Figure 5.1. RANS equations are employed in a coarse RANS domain, while LES equations are employed in a refined LES region near the TE. It's important to note that only the noise radiated by the flow within the LES region is predicted in the numerical simulations. Since the presented work is focused on TE noise predictions, it is reasonable to neglect the noise generated by other airfoil sections, such as the leading edge. All airfoil geometric parameters are shown in Table 5.1. Two embedded configurations were tested. For cases C1.1 and C1.2, the LES domains in the streamwise direction extend from $x / c=0.5$ and $x / c=0.7$, respectively, to $1 c$ downstream of the TE. The letter "C" stands for computational. The origin is defined at the airfoil leading edge. In the transverse direction, the LES domain extends $0.25 c$ above and below the airfoil. Two serration configurations are also tested. A general model of the serration characteristics is presented in Figure 5.2. Table 5.2 summarizes the flow parameters of the simulations. Computations are carried out at a free stream velocity $u_{\infty}=24 \mathrm{~m} / \mathrm{s}$ and a free stream Mach number $M_{\infty}=0.071$, resulting in a chord-based Reynolds number, $R e_{c}=\frac{\rho u_{\infty} D}{\mu}$, of approximately 500,000, where $\rho$ is the fluid density, $\mu$ is the dynamic viscosity and $D$ is the characteristic length, which is the airfoil chord in this case.

Table 5.1: Geometric parameters of simulated airfoils.

\begin{tabular}{cccccc}
\hline Case & $c(m m)$ & $s(m m)$ & $2 h(m m)$ & $\lambda(m m)$ & $\varepsilon(m m)$ \\
\hline C1.1 & 300 & 18 & - & - & - \\
C1.2 & 300 & 30 & - & - & - \\
C2.1 & 300 & 30 & 30 & 10 & 7.4 \\
C2.2 & 300 & 30 & 60 & 7.5 & 16.3 \\
C2.3 & 300 & 30 & 60 & 10 & 16.3 \\
\hline
\end{tabular}


Table 5.2: Flow parameters.

\begin{tabular}{cc}
\hline$u_{\infty}$ & $24(\mathrm{~m} / \mathrm{s})$ \\
$M_{\infty}$ & 0.071 \\
$R e_{c}$ & 500,000 \\
$\mu$ & $1.7894 \times 10^{-5}(\mathrm{~kg} / \mathrm{m} . \mathrm{s})$ \\
$\rho$ & $1.225\left(\mathrm{~kg} / \mathrm{m}^{3}\right)$ \\
$A o A$ & $0^{0}$ \\
\hline
\end{tabular}

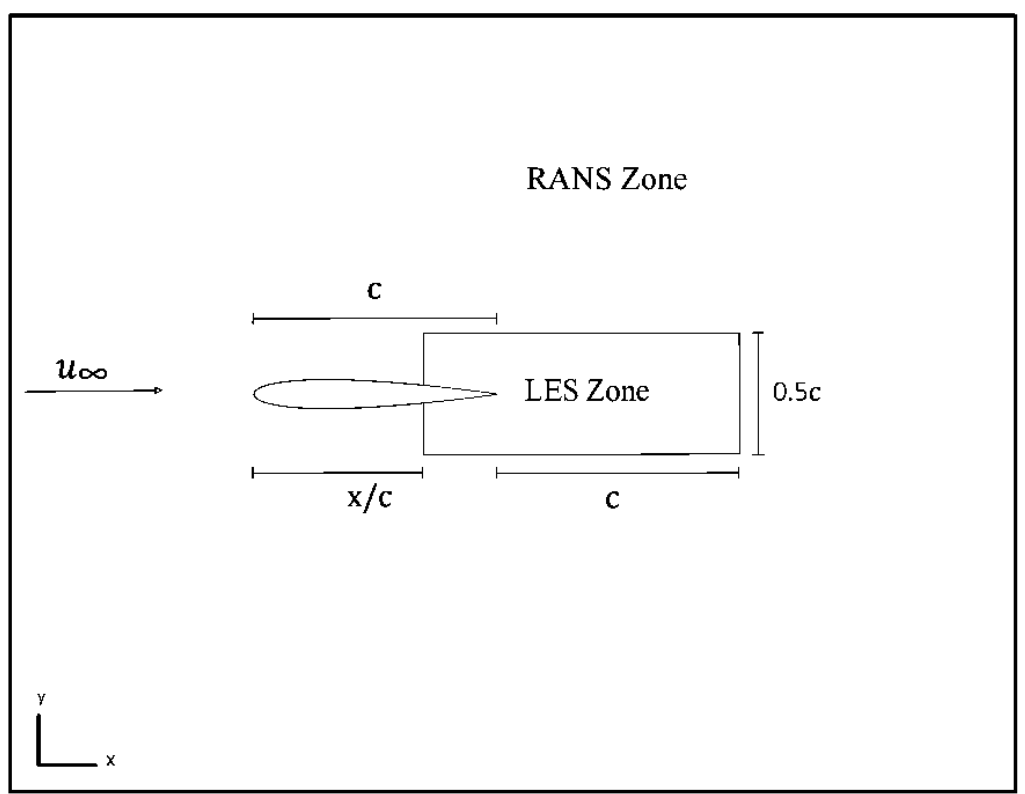

Figure 5.1: Schematic illustration of the segregated modeling domains.

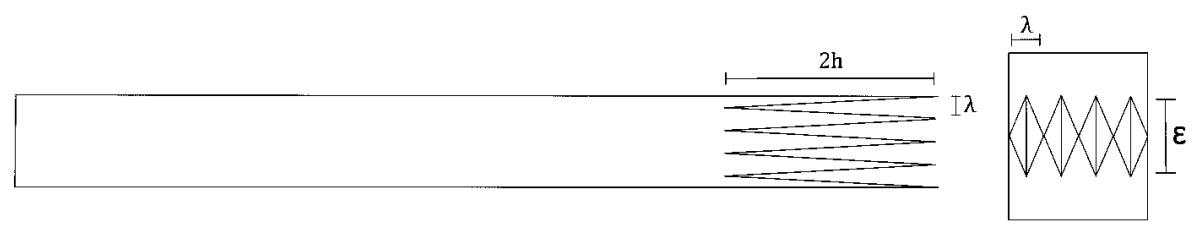

a) Top view

b) Airfoil TE

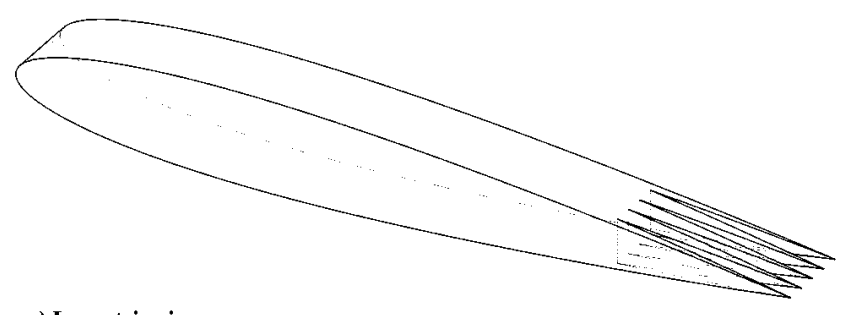

c) Isometric view

Figure 5.2: General serration configuration, not to scale. 


\subsubsection{Computational Mesh and Setup}

A predominantly hexahedral mesh is generated following the cartesian cut-cell method (Figure 5.3). This meshing technique, which has received a significant development in recent years [119], was found ideal for the current study seeing that it results in a smaller number of elements for the same resolution compared to other methods, thus significantly reducing the simulation time. In addition, the resulting elements are characterized by their high orthogonal quality and low skewness, which minimizes truncation errors [119, 120]. Element size is restricted to $25.6 \mathrm{~mm}$ in the coarse RANS zone, $0.8 \mathrm{~mm}$ in the refined LES zone and $0.2 \mathrm{~mm}$ on the airfoil surface in the vicinity of the TE. Elements in the airfoil wake of the RANS zone have a size of $5 \mathrm{~mm}$. The grid resolution in terms of wall-normal units is defined by $\Delta x^{+}=\frac{u_{t} \Delta x}{\nu}, \Delta y^{+}=\frac{u_{t} \Delta y}{\nu}$ and $\Delta z^{+}=\frac{u_{t} \Delta z}{\nu}$, where $u_{\tau}$ is the frictional velocity and $\nu$ is the kinematic viscosity. 40 inflation layers (Figure 5.3p) are generated around the airfoil with the thickness of the first layer set to $7 \times 10^{-3} \mathrm{~mm}$ and a growth factor of 1.08, thus ensuring $y^{+}<0.5$ everywhere on the airfoil surface (Figure 5.4), at least 3 layers in the viscous sublayer and overall accurate boundary layer resolution. Table 5.3 lists mesh statistics for all simulated cases. The computational grid has a maximum resolution $\Delta x_{\max }^{+} \leq 20$ and $\Delta z_{\max }^{+} \leq 20$ in the streamwise and spanwise directions, respectively [121, 122]. Case C2.3 is simulated using two different meshes to investigate the effect of the mesh on the predicted tonal peak. A steady-state mesh convergence study was carried out by progressively refining the mesh, creating three meshes having $6,666,668$ elements, 7,606,083 elements and 9,011,531 elements respectively. The values of integrated output parameters, such as lift and drag coefficients, were compared and the maximum error is found to be less than $0.4 \%$, demonstrating mesh convergence. Furthermore, the first two meshes were carried over for a transient simulation analysis. The lift-history coefficients were evaluated for each mesh at every time step and their RMS values were

computed. Both meshes yield the same lift-coefficient RMS value, $c_{L_{R M S}}=0.0013$ and Strouhal number, $S t=0.168$. Consistent results in terms of integrated flow parameters, 
for both steady-state and transient simulations, are a strong indication of the convergence of the used computational mesh, i.e. the mesh directly resolves enough flow structures for the results not to change with mesh refinement.

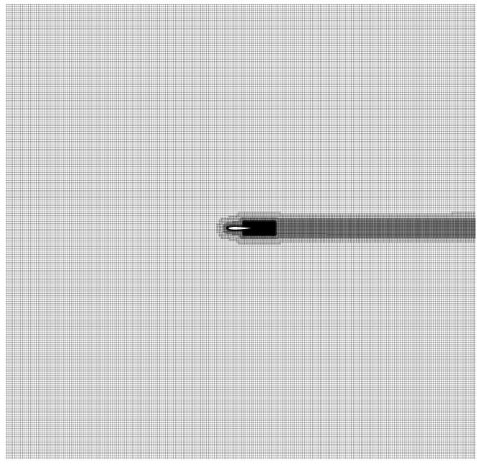

a) Mesh overview

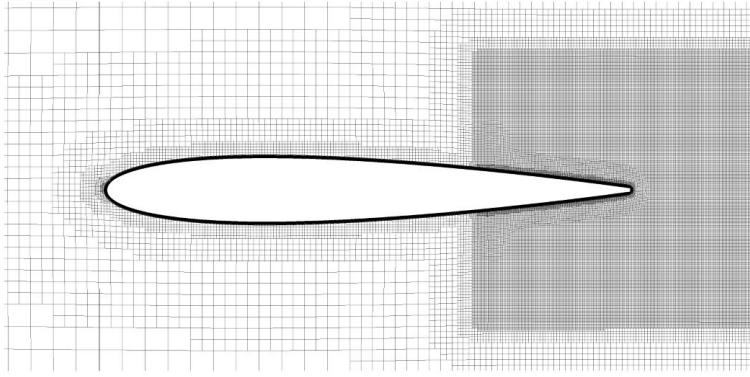

b) Mesh near airfoil surface

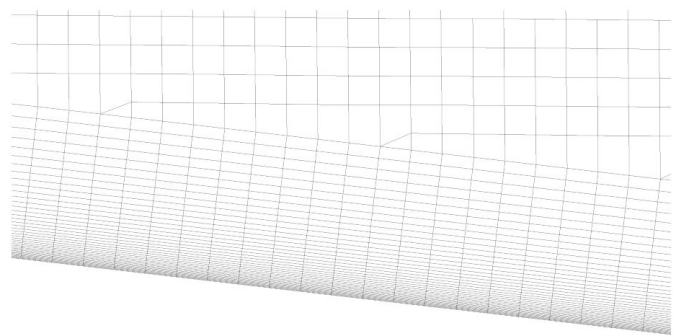

c) Inflation layers around airfoil surface

Figure 5.3: Computational mesh. 

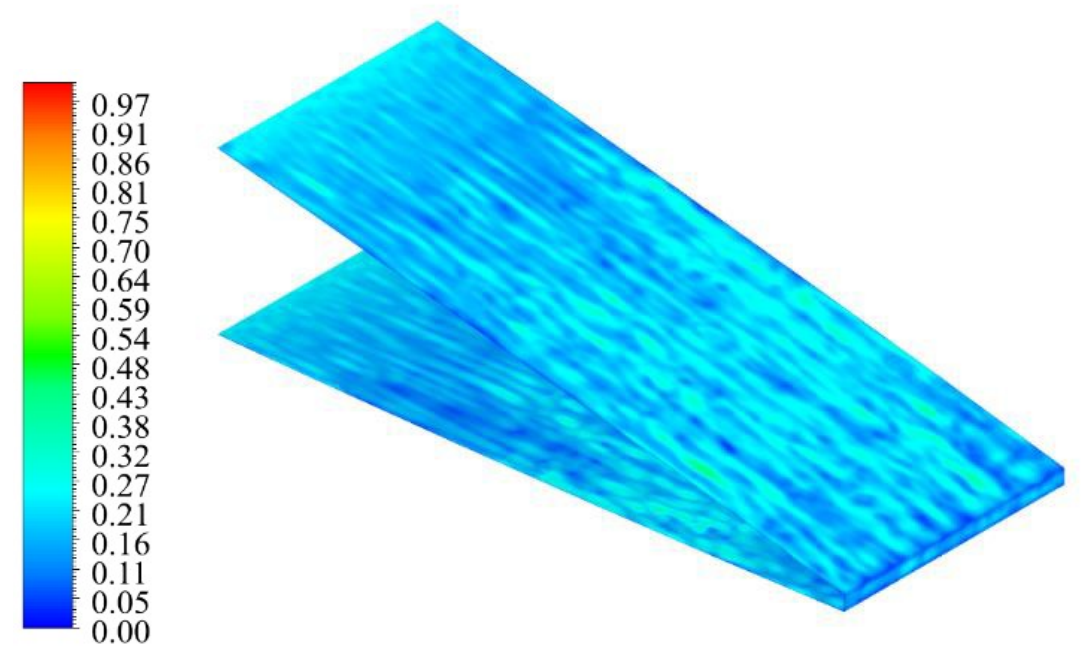

Figure 5.4: Instantaneous $y+$ distribution.

Table 5.3: Mesh elements.

\begin{tabular}{lccc}
\hline \multicolumn{1}{c}{ Case } & RANS & LES & Total \\
\hline C1.1 & 456,353 & $4,586,780$ & $5,043,133$ \\
C1.2 & 976,300 & $5,690,368$ & $6,666,668$ \\
C2.1 & 976,300 & $6,134,899$ & $7,111,198$ \\
C2.2 & 976,300 & $5,896,422$ & $6,872,722$ \\
C2.3, Mesh1 & 976,300 & $5,647,833$ & $6,624,133$ \\
C2.3, Mesh2 & $1,050,433$ & $6,173,725$ & $7,224,158$ \\
\hline
\end{tabular}

The boundary conditions used are demonstrated in Figure 5.5. A velocity inlet boundary condition is specified at the domain entrance, where $u_{\infty}=24 \mathrm{~m} / \mathrm{s}$. Periodic boundary conditions (PBCs) are applied on the right and left side walls of the domain in the spanwise direction to allow the flow to develop naturally. No-slip boundary conditions are applied on the airfoil surface and a zero gauge-pressure outlet boundary condition is used. The inlet turbulence is set to $0.3 \%$. The SIMPLE pressure-velocity coupling scheme is used. All results are second order accurate in time and space.

The flow is initialized using the $k-\omega S S T$ model developed by Menter [123], as it gives accurate separation predictions for external flows. The VM is then used to inject 
turbulence at the RANS/LES interface and the simulation is run for $4 T_{T F}$ "Through-flow time" to obtain a fully developed flow, where $T_{T F}=L_{C F D} / u_{\infty}$ 121. $L C F D$ is the LES domain length in the streamwise direction. WALE subgrid-scale (SGS) modelling is employed in the LES region as it is designed to return correct asymptotic wall behavior for wall-bounded flows [122]. The time step $d t=1.2 \times 10^{-5}$ seconds. With these values, the Courant-Friedrichs-Lewy (CFL) number achieved is $\leq 1$ everywhere in the domain, meaning the flow particles don't travel more than the length of one mesh element every time step. Residuals are reduced by three orders of magnitude each time step. Lastly, acoustic data is gathered for $3 T_{T F}$. All convergence residuals are set to $10^{-5}$. Pressure and velocity monitoring points were placed in the airfoil wake and statistical convergence is achieved. Statistical convergence is also achieved for the coefficients of lift and drag. All simulations are carried out using the commercial CFD software FLUENT 2019R3 and run on Intel Xeon L5410 $2.33 \mathrm{GHz}$ platform of 60 cores.

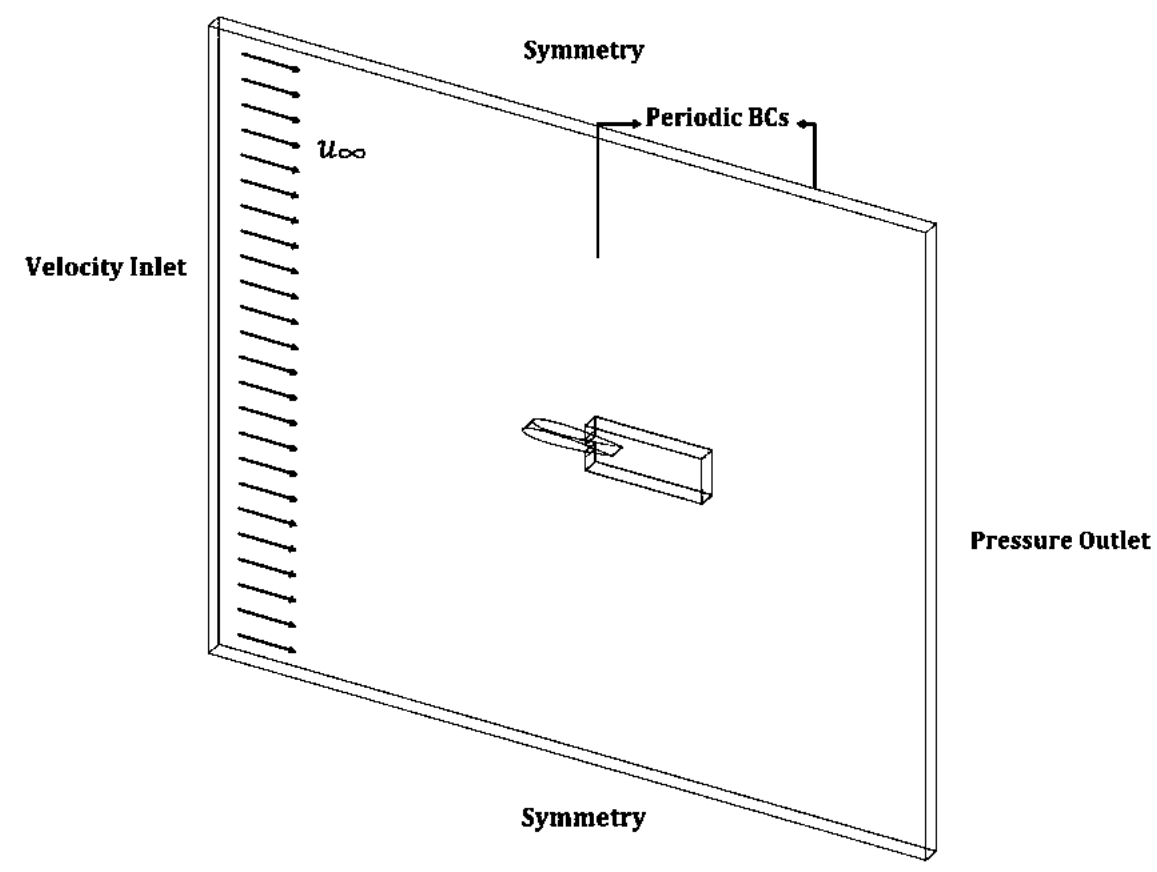

Figure 5.5: Boundary Conditions. 


\subsubsection{Experimental Setup}

Experiments were conducted in the medium-speed, subsonic, closed-loop wind tunnel at Carleton University (Figure 5.6). The airflow is powered by a $37.3 \mathrm{~kW}(50 \mathrm{HP}$ ) variablespeed DC motor driving a $1.2 \mathrm{~m}$ axial propeller at speeds as high as 900 RPM. A variable frequency drive (VFD) modulates the rotational frequency of the fan at a resolution of 1.0 Hz. A series of turbulence grids precede a 9:1 contraction, which reduces the turbulence intensity levels in the center of the test section to less than $0.27 \%$, as measured for speeds up to $15 \mathrm{~m} / \mathrm{s}$ (as shown in Figure 5.6). The tunnel has a removable, rectangular test section along with the surrounding anechoic chambers was completed to be used for aeroacoustic testing. This test section is a $0.78 \mathrm{~m} \times 0.51 \mathrm{~m}$ rectangular section, $1.83 \mathrm{~m}$ long. The upper and lower walls of the test section are each composed of two aluminum sheet panels and contain hardware (circle aluminum material) for the vertical mounting of a two-dimensional airfoil in the midway, and $0.45 \mathrm{~m}$ from the upstream end of the test section 28,29 .

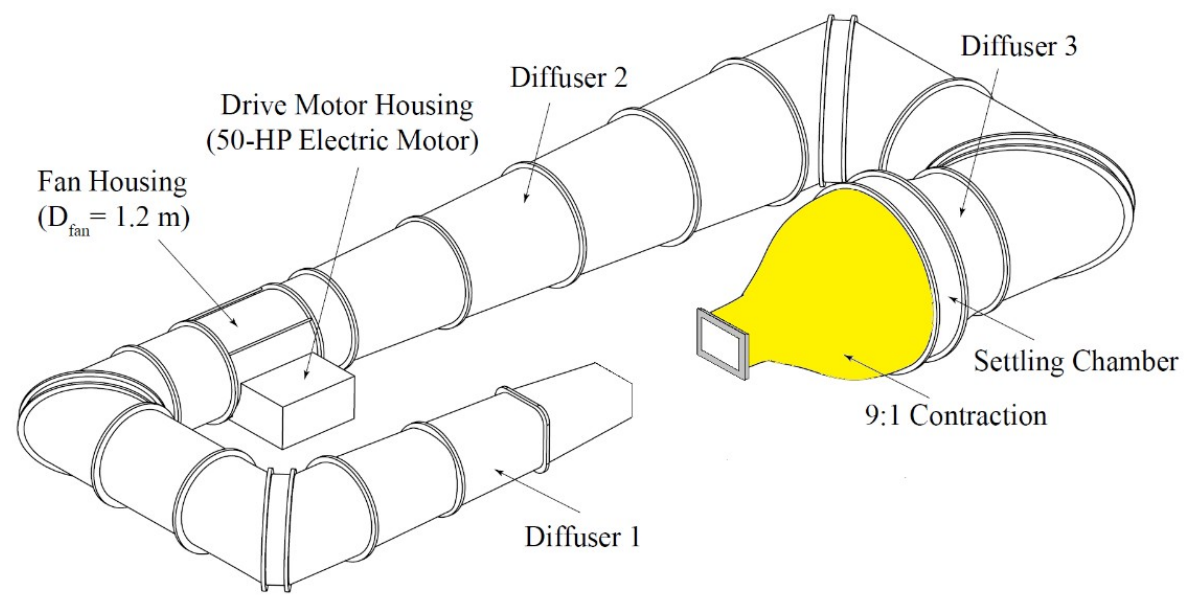

Figure 5.6: Wind tunnel configuration at Carleton University. 


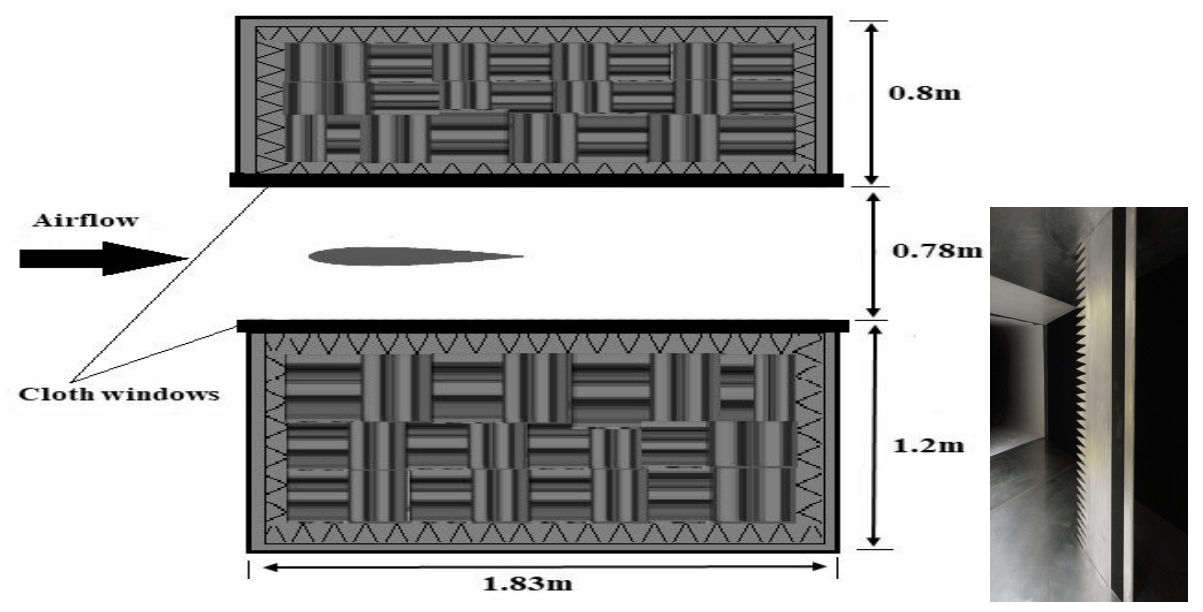

Figure 5.7: Cross section through the aeroacoustic test section and anechoic chamber as seen from above (left) and photograph of serrated TE airfoil in test section (right).

The airfoil wing is mounted vertically in the test section (Figure 5.7) with its leading edge (at zero AoA) $0.45 \mathrm{~m}$ downstream of the test section entrance. The airfoil under investigation is a NACA-0012 airfoil with a sawtooth TE serration cut directly into the main body of the airfoil (as shown in Figure 5.8). The chord length of the airfoil is 300 $\mathrm{mm}$, and the width is $510 \mathrm{~mm}$. Between the leading-edge $\mathrm{x} / \mathrm{c}=0$, and $\mathrm{x} / \mathrm{c}=0.73 \mathrm{~mm}$, the original NACA-0012 airfoil profile is unmodified, where $x$ is the streamwise direction. Further downstream, $0.73 \leq x / c \leq 1.0$, is a section that can be removed and replaced by either an unmodified or modified TE profile. Once attached, the TE section forms a continuous profile giving the appearance that the serrations are cut into the main body of the NACA-0012 airfoil. Typical parameters including the serration amplitude, $2 h$, and serration wavelength, $\lambda$, are defined as specified in Figure 5.2. A prominent feature for airfoil that this type of serrated TE is the exposure of a significant bluntness $\varepsilon$ at the root region. A photograph of the sawtooth serrated TEs used is shown in Figure 5.8 .

Table 5.4 shows the summary of geometrical parameters of the two TE serration tested in the present paper, according to $2 h, \lambda$ and $\varepsilon$, in which E0 represents the baseline sharp trailing-edge. The letter "E" stands for experimental. Far-field noise measurements in the 
mid-span were performed by a calibrated Bruel \& Kjaer microphone, which is installed at a distance of $1.4 \mathrm{~m}$ for an observer angle $\alpha=90^{\circ}$. The analysis was carried out between $100 \mathrm{~Hz}$ and $5 \mathrm{kHz}$.

Table 5.4: Experimental geometric parameters.

\begin{tabular}{cccccc}
\hline Case & $c(m m)$ & $s(m m)$ & $2 h(m m)$ & $\lambda(m m)$ & $\varepsilon(m m)$ \\
\hline E0 & 300 & 500 & - & - & - \\
E1 & 300 & 500 & 70 & 25 & 18.2 \\
E2 & 300 & 500 & 60 & 10 & 16.6 \\
\hline
\end{tabular}

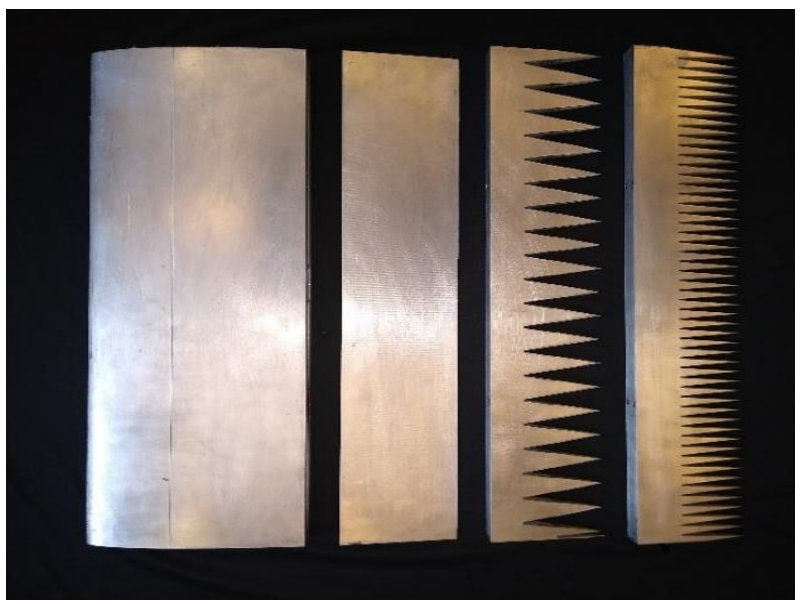

Figure 5.8: From left to right: NACA-0012 main body, TE of case E0, TE of case E1 and TE of case E2.

\subsection{Results}

\subsubsection{Surface Pressure}

The pressure coefficient distribution around the airfoil is an especially important pa- 
rameter, both in terms of aerodynamic characteristics, since it determines the lift coefficient, and in terms of boundary layer behavior, since the pressure gradient is known to affect the development of both laminar and turbulent boundary layers $[124-126$. In addition, the BL is responsible for the majority of the generated sound. $C_{p}$ distributions for cases C1.1 and C1.2 are computed for validation and compared against experimental results obtained by Lee and Kang 127 for a NACA-0012 airfoil at a $R e_{c}=600,000$, and full LES results published by Marsden, Bogey and Bailly $\mid 128$ at $R e_{c}=500,000$ (Figure5.9). Excellent agreement is found between the computational and experimental results. Of importance is the fact that from $x / c=0.15$ down to the TE, the boundary layer is subject to an adverse pressure gradient. Both cases $\mathrm{C} 1.1$ and $\mathrm{C} 1.2$ are validated against existing literature. The LES domain in C1.1 is longer in the streamwise direction as it starts at a $x / c=0.5$, while it starts at $\mathrm{x} / \mathrm{c}=0.7$ in $\mathrm{C} 1.2$ (see Tables 5.1 and 5.3). Even though both configurations yield acceptable results, the configuration of case C1.2 is chosen for the succeeding simulations as the LES domain covers a larger span with a reasonable increase in computational time.

Figure 5.10 shows the location of maximum pressure fluctuation, where $P_{R M S}$ is maximum for cases C1.2, C2.1 and C2.2. For case C1.1, the location of maximum $P_{R M S}$ is close to the sharp TE, seeing that that is where the discontinuity occurs and the BLs on the pressure and suction sides clash. The introduction of serrations concentrated the maximum pressure fluctuation region from the extremity of the TE to downstream of the serration roots. This is where the pressure fluctuations are most violent, which suggests that aeroacoustic improvement to this design would require a modification of the flow field at that area, because regions with the highest $P_{R M S}$ emit the most noise. The presence of serrations mitigates the sudden interaction between the BLs on the pressure and suction sides, allowing for progressive mixing and affecting the radiated sound. By comparing the maximum $P_{R M S}$ values for $\mathrm{C} 2.1, \mathrm{C} 2.2$ and $\mathrm{C} 2.3$, the maximum $P_{R M S}$ increases as the serration amplitude, and subsequently the root bluntness, are increased. C2.2 and 
C2.3 share the same root bluntness, $\epsilon$, and serration amplitude, $2 h$, and are subject to comparable $P_{R M S}$ values.

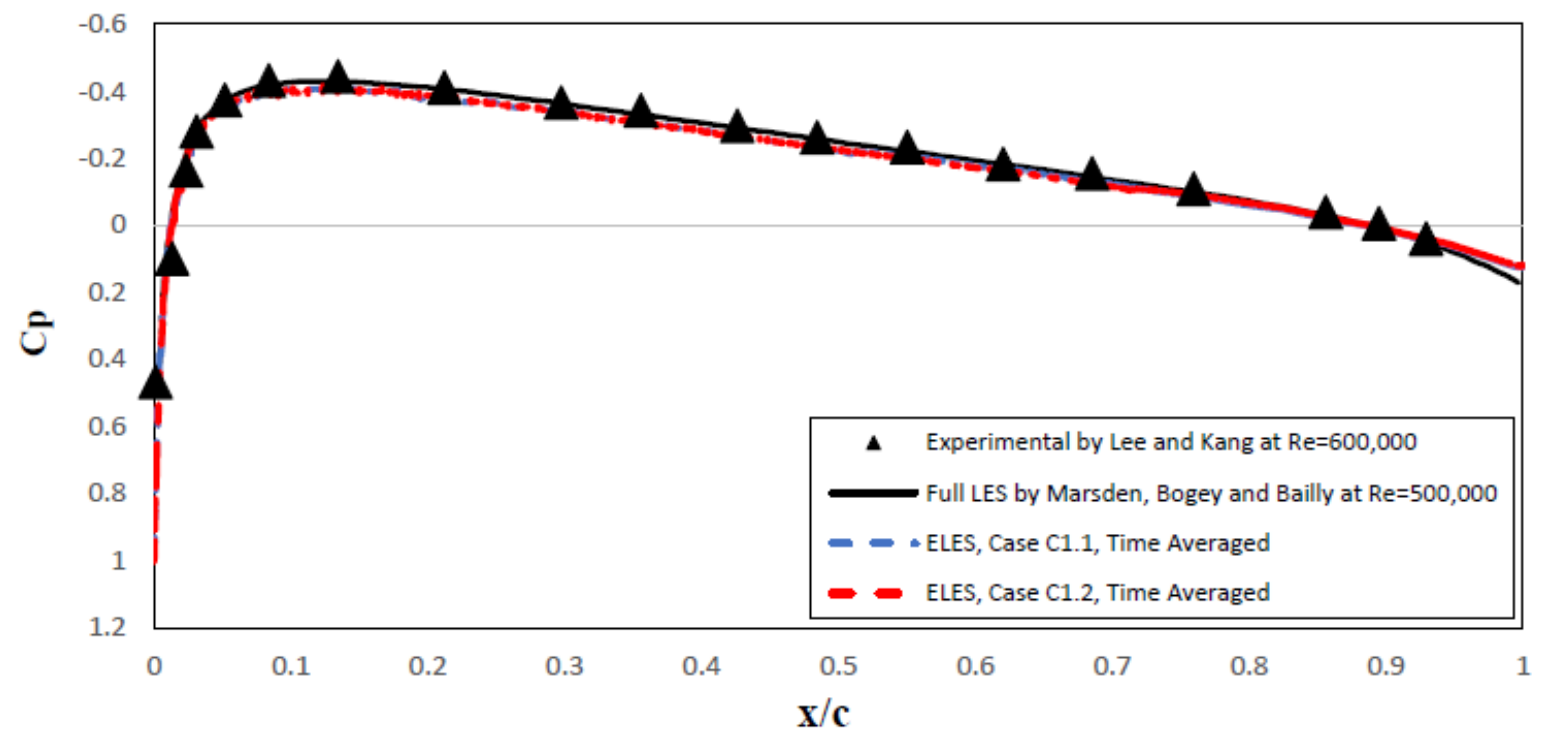

Figure 5.9: $C_{p}$ distribution on airfoil surface.
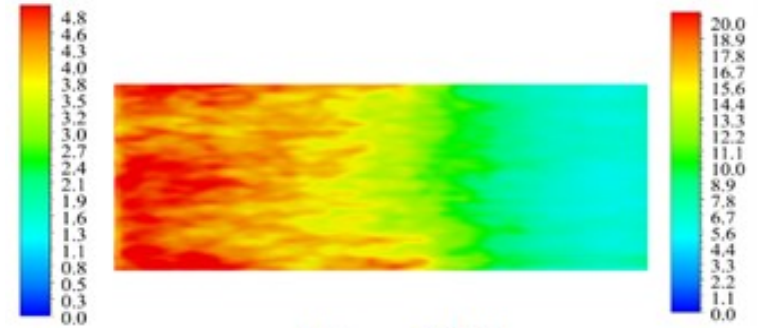

a) Case $\mathrm{C} 1.2$

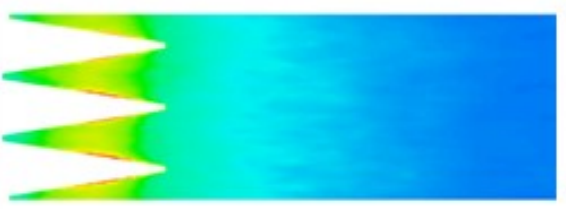

b) Case C2.1
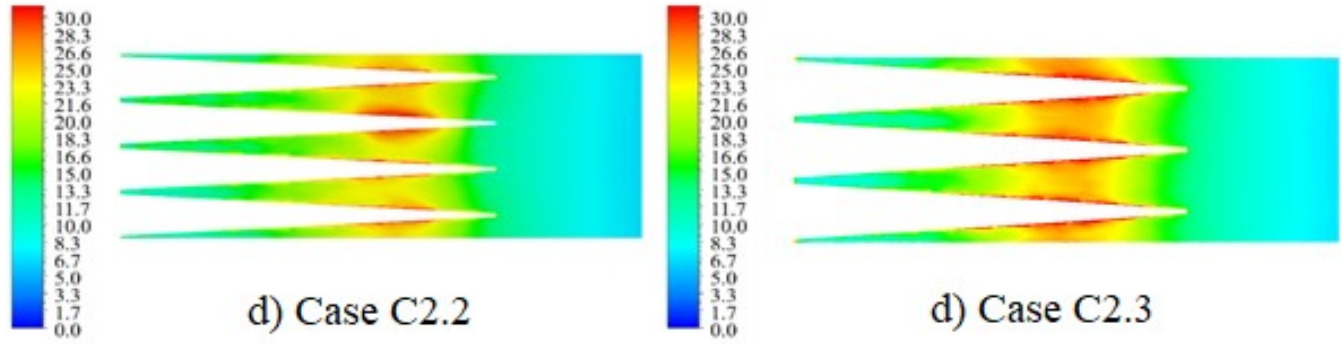

d) Case C2.3

Figure 5.10: $P_{R M S}$ distribution near airfoil TE. 


\subsubsection{Wake Characteristics}

By calculating the coefficient of lift, $C_{L}=\frac{L}{0.5 \rho \nu^{2} A^{\prime}}$, for every timestep of flow simulation, the lift-coefficient history can be plotted. $L$ is defined as the lift force and $A$ is the airfoil area. The lift-coefficient history is commonly used as an indicator of statistical convergence in transient simulations. Furthermore, it's a non-dimensional representation of the fluctuating forces acting normal to the airfoil surface due to the turbulence of air flow. To demonstrate the convergence of the computational mesh, C2.3 is simulated twice, using two different meshes having 6,624,133 and 7,224,158 elements, respectively. The obtained lift-coefficient history plots are presented in Figure 5.11. Both simulations yield the same lift-variation amplitude and frequency once the flow is initialized. The only observable difference is a phase shift, which is normal and simply means each simulation started from a different point in the periodic cycle. Both simulations also predict the same Strouhal number, $S_{t}=0.168$. Figure 5.12 shows the lift-coefficient history plots of all the presented cases. All axes are kept constant and aligned for the sake of clarity and comparison. For case C1.2, the lift monitor is random and irregular, characterized by a relatively small amplitude. With the introduction of serrations, the lift monitors adopt sinusoidal shapes having different wavelengths and frequencies. C2.1 is characterized by the smallest amplitude and highest frequency, $f=388 \mathrm{~Hz}$. C2.2 and C2.3 are almost subject to the same fluctuation amplitude, but their lift-coefficients vary with distinct frequencies equal to $218 \mathrm{~Hz}$ and $248 \mathrm{~Hz}$, respectively. This behavior is attributed to the vortices shed in the wake of the airfoil. The dominant frequencies of the periodic plots were obtained by applying discrete Fast-Fourrier Transform (FFT) on the propagated presented lift-coefficient history plots. Figure 5.13 shows the instantaneous flow fields in the airfoil wake in term of isosurfaces of the Q-criterion, which is defined as the 
second invariant of the instantaneous velocity gradient tensor [129]. The iso-surfaces are used to identify and portray the turbulent coherent structures of the wake, which are inherently three-dimensional. The isosurfaces are colored by the spanwise vorticity $\omega_{z}$ and demonstrate how the wake behavior changes as standard serrations are introduced then their geometrical parameters modified. For the case of a flat TE (C1.2), the wake is non-uniform and has almost no observable coherent structures, while serrated cases (C2.1, C2.2 and C2.3) are clearly subject to vortex shedding.

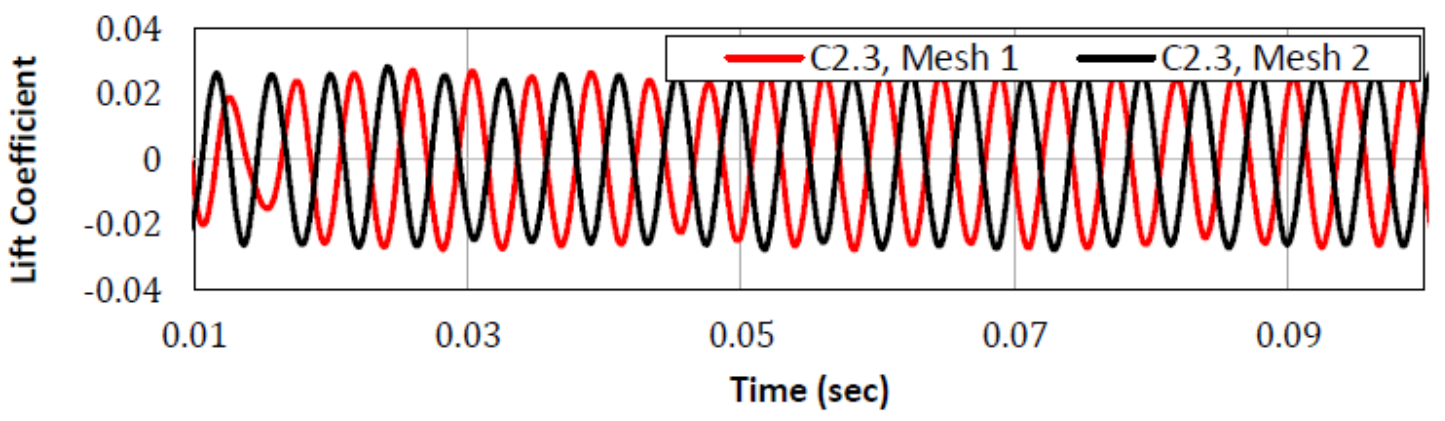

Figure 5.11: Lift-coefficient history of C2.3 using two different meshes.

For the case of a flat TE (C1.2), the wake is turbulent but has no identifiable coherent structures. For the cases of standard serrations (C1.2, C2.2 and C2.3), the wake is characterized by sinusoidal vortex shedding. The amplitude and frequency of the observed phenomenon change as the serrations amplitude and wavelengths are varied. C2.1 is subject to the highest shedding frequency and the smallest amplitude. C2.2 and C2.3 are subject to similar vortex shedding amplitudes, but the frequency is higher in $\mathrm{C} 2.3$. The vortex shedding frequency can also be approximated by counting the number of full periodic cycles in the airfoil wake and dividing it by the through-flow time $(T T F)$. Vortex shedding is caused by the interaction of two shear layers as they leave the airfoil surface. Because of the inherent instability of the turbulent boundary layer, alternating low pressure zones are generated downstream of the airfoil, giving rise to fluctuating forces acting normal to the wind direction, which in turn explains the sinusoidal lift coefficient variation 
(Figure 5.12). The frequencies at which vortices are shed from the airfoil TEs are equal to those at which the non-dimensional lift forces acting on the airfoil vary, and can thus be accurately calculated by applying a discrete FFT on the lift-coefficient history plots. Then, cases C2.1, C2.2, C2.3 and C4 are subject to vortex shedding frequencies of $388 \mathrm{~Hz}$, $218 \mathrm{~Hz}, 248 \mathrm{~Hz}$ and $210 \mathrm{~Hz}$, respectively. A generated vortex is initially growing and fed by circulation from the separated shear layer, until it becomes strong enough to roll up and draw the opposing shear layer across the wake. At that point, this vorticity of opposite sign interrupts any further supply of circulation to the growing vortex, which then stops increasing in strength. As a result, that vortex is shed and convected downstream while a new one of opposite vorticity takes its place and the cycle keeps going 130,131. As the serration amplitude $(2 h)$ is reduced, the root bluntness $(\varepsilon)$ is also reduced and the shear layers are brought closer together. Subsequently, the interaction between the two shear layers is facilitated and the periodic time is shortened, giving rise to a higher vortex shedding frequency [49]. 

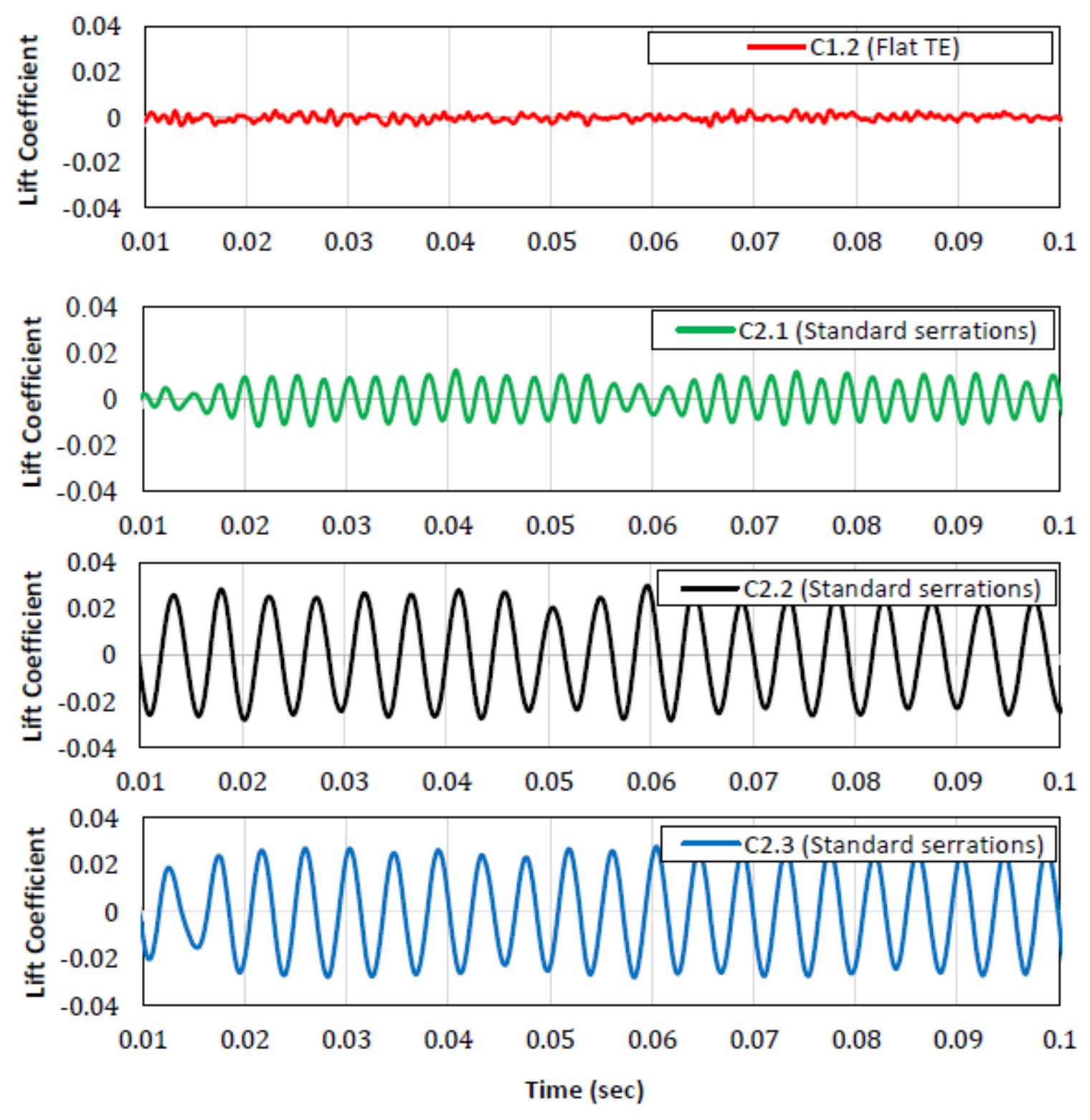

Figure 5.12: Lift-coefficient history for case C1.2, C2.1, C2.2 and C2.3. 


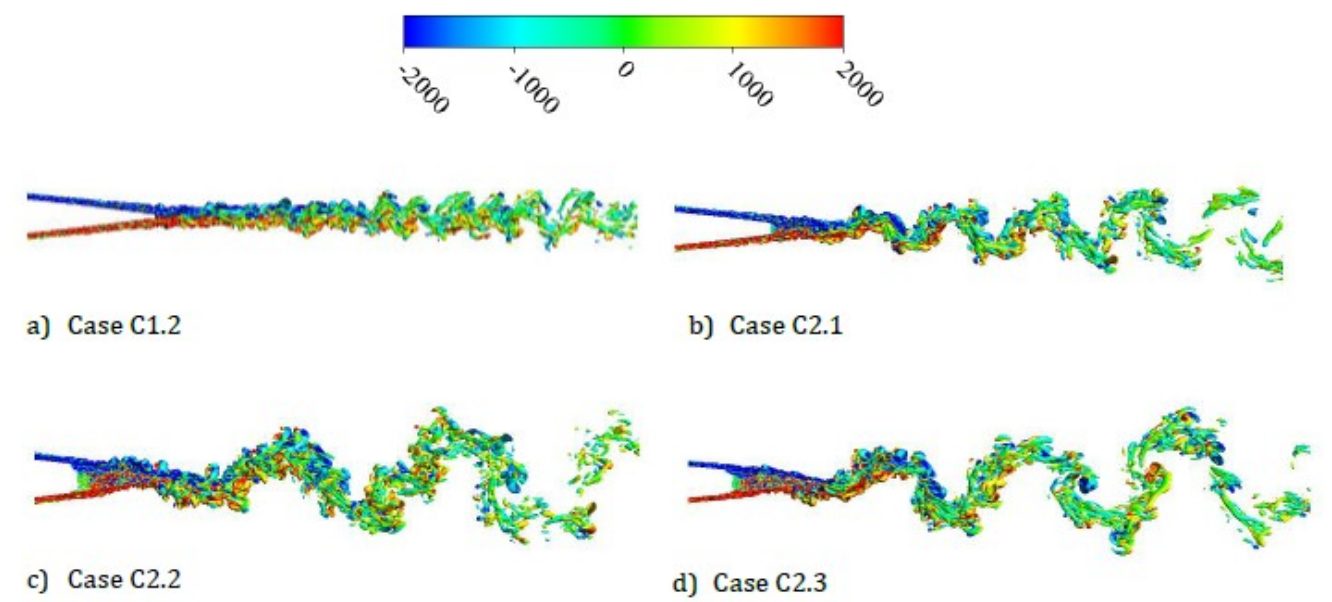

Figure 5.13: Instantaneous Q-Criterion colored by $\omega_{z}$.

In order to study the dynamics of vortex shedding, the Strouhal number, $S t=\frac{f_{S} L}{u_{\infty}}$, is often used [132, in which $f_{s}$ is the vortex shedding frequency in $\mathrm{Hz}, \mathrm{L}$ is the characteristic length separating the shear layers in meters and $u_{\infty}$ is the free-stream velocity. St represents the ratio of inertial forces due to the local acceleration of the flow to the inertial forces due to the convective acceleration. The first is a product of turbulence and how the velocity of a fluid particle changes due to the inherent instability of the TBL, while the latter is an indication of how much the velocity changes as the flow moves across the fluid domain. St is particularly helpful for flows characterized with periodic motion as it associates the oscillations of the flow due to the inertial forces to the changes in velocity due to the convective acceleration of the flow field. In the case of a flat TE, the oscillations are not prominent, seeing that they are swept by the fast-moving fluid (Figure $5.13 \mathrm{a})$. When changing the serration amplitude $(2 \mathrm{~h})$ from $30 \mathrm{~mm}$ (C2.1) to $60 \mathrm{~mm}$ (C2.2), the observed vortex shedding frequency (Figures 5.13b and 5.13 d) is reduced and $S t$ increases $\left(S t_{2.1}=0.119\right.$ and $\left.S t_{2.3}=0.168\right)$. Increasing $\lambda$ while keeping $2 h$ constant leads to an increase in vortex shedding frequency and $S t\left(S t_{2.2}=0.148\right.$ and $\left.S t_{2.3}=0.168\right)$. The observed trend is in good agreement with the work of $\mathrm{Hu}$ at al. [133], as well as the 
aforementioned findings. Different airfoils will have different root bluntness for the same serration amplitude depending on their profile, and subsequently different vortex shedding frequencies. Wake vorticity is also dissipated faster in cases C2.3 and C2.2 than C2.1 and $\mathrm{C} 1.2$, which can be seen by inspecting the vorticity magnitude in the wake, shown in Figure 5.14. Lastly, Figure 5.15 gives the streamwise vorticity, $\omega_{x}=\frac{\partial u_{y}}{\partial z}-\frac{\partial u_{z}}{\partial y}$, contours for all the simulated cases. The two limits of the contour correspond to fluid particles having equal vorticity but in opposite directions. For the case of a flat TE (C1.2), turbulent, counter-rotating coherent structures are observed at the TE. For the cases having standard serrations (C2.1, C2.2 and C2.3), the turbulent coherent structures are allowed to pass between the serrations, across the airfoil surface.

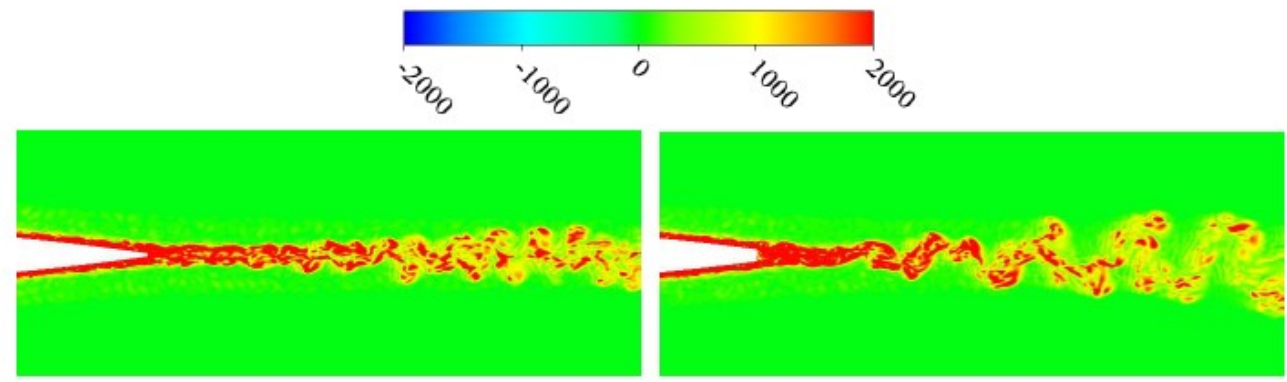

a) Case $\mathrm{C} 1.2$

b) Case $\mathrm{C} 2.1$
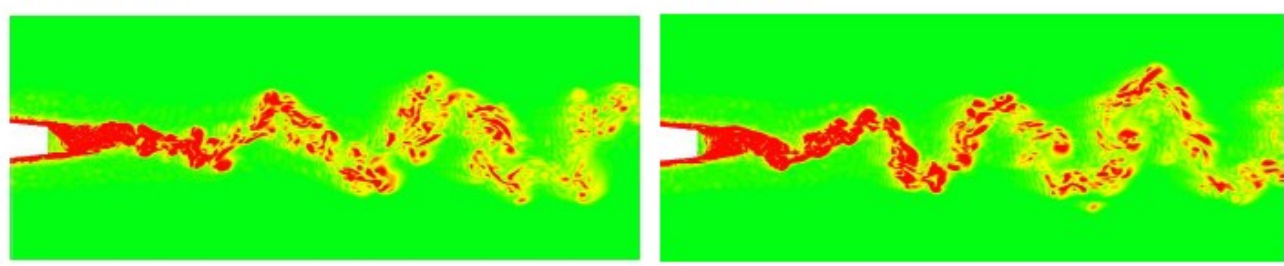

c) Case $\mathrm{C} 2.2$

d) Case $\mathrm{C} 2.3$

Figure 5.14: Instantaneous vorticity magnitude contours. 


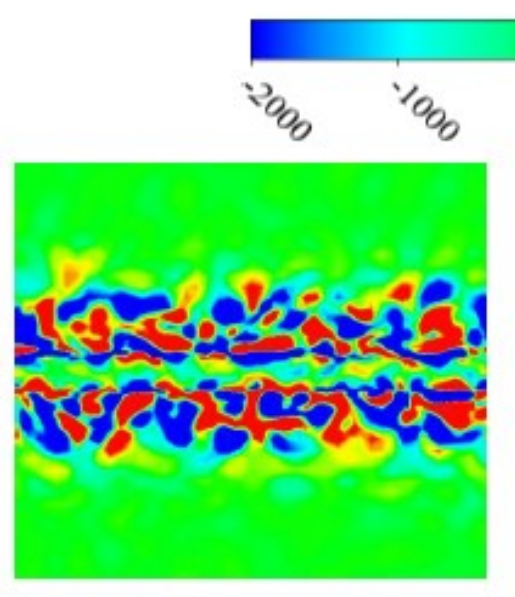

a) Case $\mathrm{C} 1.2$

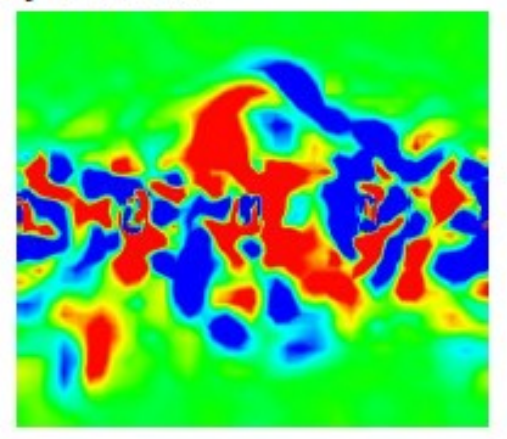

d) Case C2.2

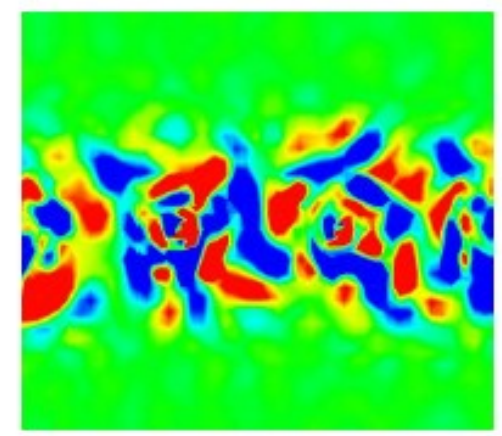

b) Case $\mathrm{C} 2.1$

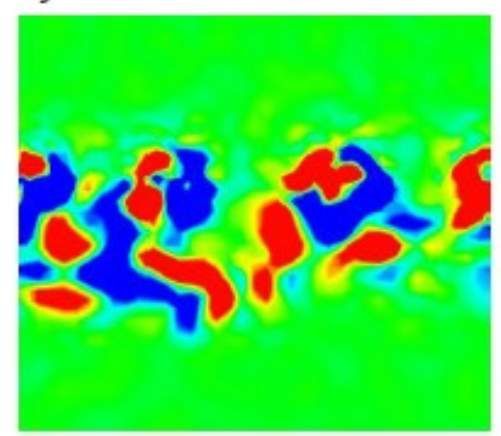

d) Case C2.3

Figure 5.15: Instantaneous $\omega_{x}$ contours at $x / c=1$.

\subsubsection{Far-field Noise}

The FW-H aeroacoustic analogy [7, 117] is used to compute the radiated far-field noise for the computational cases C1.2, C2.1 and C2.2. Pressure fluctuations are propagated to receivers placed midspan at a distance of 1.5 meters directly above the airfoils' TEs. Acoustic data sampling is performed for $3 T_{F T}$ after the flow is fully developed, and the acoustic data is sampled every 2 flow-timesteps, resulting in a sampling frequency of $41.67 \mathrm{kHz}$ and a frequency resolution of $28.4 \mathrm{~Hz}$, where the frequency resolution is defined as the inverse of the sampling period. FFT is performed on the resulting time signals seen in Figure 5.15 to compute the sound pressure level $(S P L)$ signal in the frequency domain, shown in Figure 5.16. The Hanning window is applied to the time signal to reduce 
numerical leakages associated with the discrete FFT [134]. Tonal peaks are observed at $198 \mathrm{~Hz}$ and $397 \mathrm{~Hz}$ for cases $\mathrm{C} 2.1$ and C2.2, respectively, while case C1.2 only exhibits broadband behavior, which is caused by the incoherence of the flow in the airfoil wake. The narrowband peaks are justified by the vortex shedding caused by the serration root bluntness discussed in subsection B.

The FW-H aeroacoustic analogy [7, 117] is used to compute the radiated far-field noise for the computational cases $\mathrm{C} 1.2, \mathrm{C} 2.1, \mathrm{C} 2.3$. In order to keep the computational cost reasonable, the span of the simulation domains is kept smaller than that of the experimental testing. Acoustic data is sampled every 2 flow-timesteps and data sampling is performed for $3 T_{F T}$ after the flow is fully developed, resulting in a sampling frequency of $41.67 \mathrm{kHz}$ and a frequency resolution of $28.4 \mathrm{~Hz}$, where the frequency resolution is defined as the inverse of the sampling period. Pressure fluctuations are propagated to receivers placed midspan at a distance of 1.5 meters directly above the airfoils' TEs, as seen in Figure 5.16. Cases C2.1, C2.2 and C2.3 show periodic patterns, where the amplitude is highest in C2.2 and C2.3, and the periodic frequency is highest in C2.1. Discrete FFT is performed on the resulting time signals seen to compute the sound pressure level (SPL) signal in the frequency domain, as shown in Figure 5.17. The Hanning window is applied to the time signal to reduce numerical leakages associated with the discrete FFT [134]. Case C1.2 only exhibits broadband behavior, which is a sign of the streamwise incoherence of the flow in the airfoil wake. Tonal peaks are observed for C2.1, C2.2 and C2.3 at $397 \mathrm{~Hz}$, $198 \mathrm{~Hz}$ and $240 \mathrm{~Hz}$, respectively. The tonal peak amplitudes are equal for C2.2 and C2.3. The peak amplitude is $3.3 \mathrm{~dB}$ lower in $\mathrm{C} 2.1$, meaning the tonal noise is louder for the cases having longer serrations. The narrowband peaks are fundamentally justified by the vortex shedding caused by the serration root bluntness discussed in subsection 4.2 [135]. Figure 5.18 presents the far-field spectra obtained from simulating design C2.3 using two meshes, as previously mentioned. Both simulations predict the same narrow-band tonal peak amplitude and frequency, as well as comparable broad-band behavior at frequencies 
higher than the tonal peak.
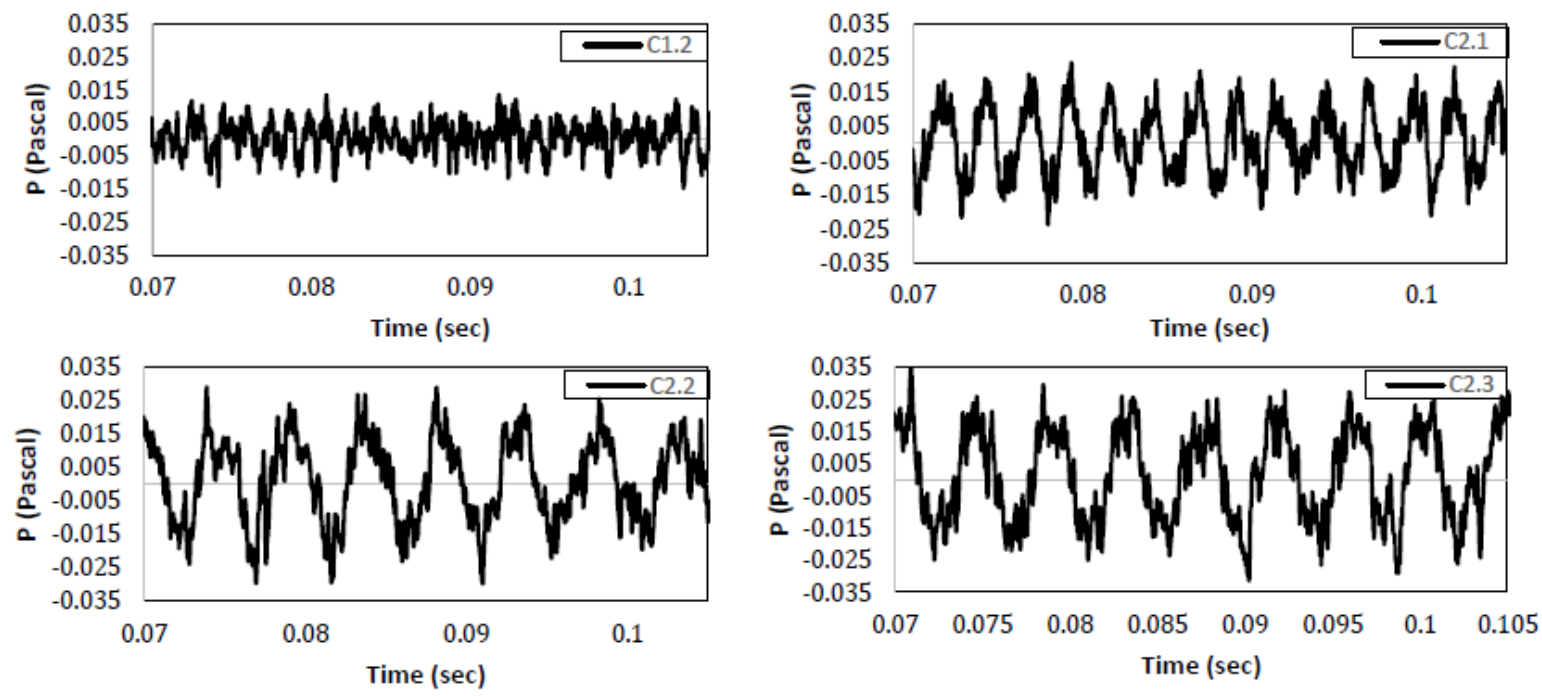

Figure 5.16: Time domain noise signals propagated to receiver.

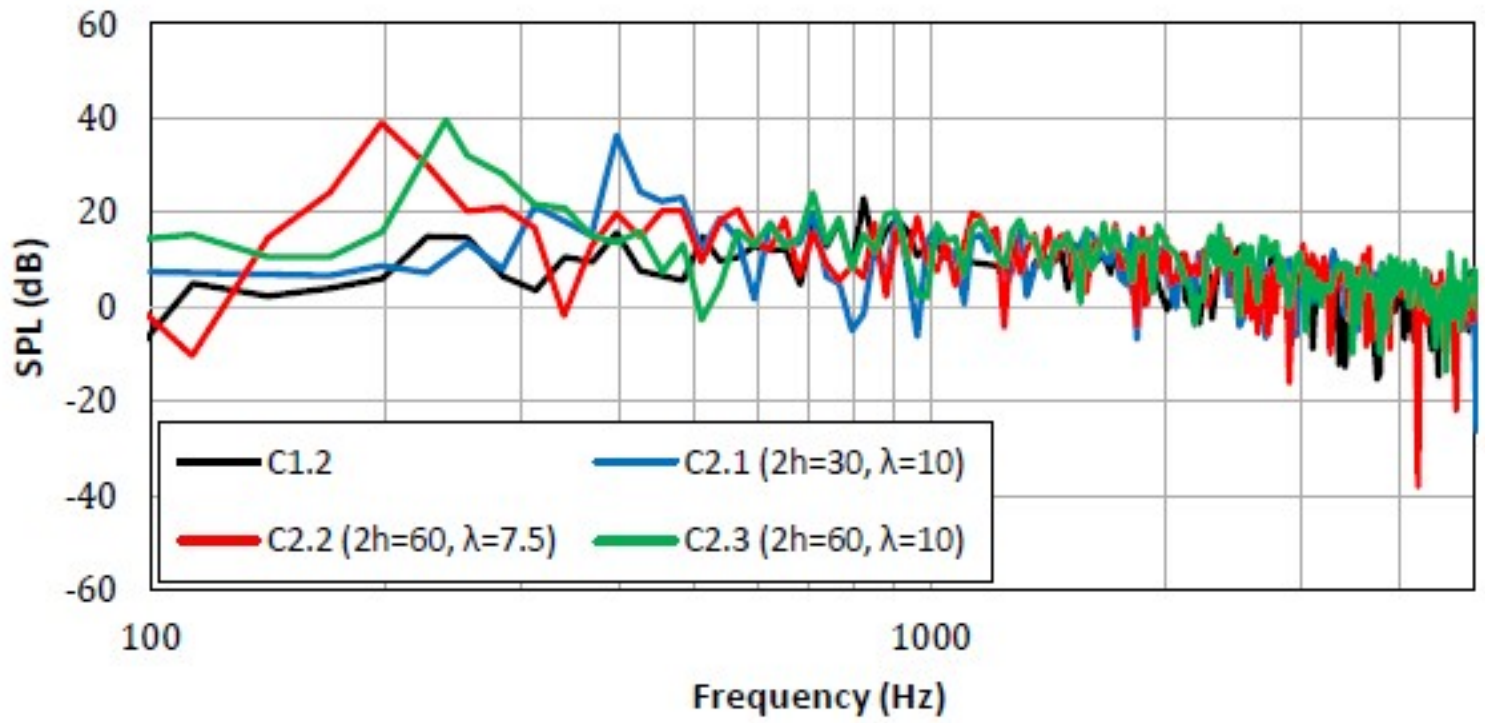

Figure 5.17: Frequency domain noise signals of C1.2, C2.1, C2.2 and C2.3 


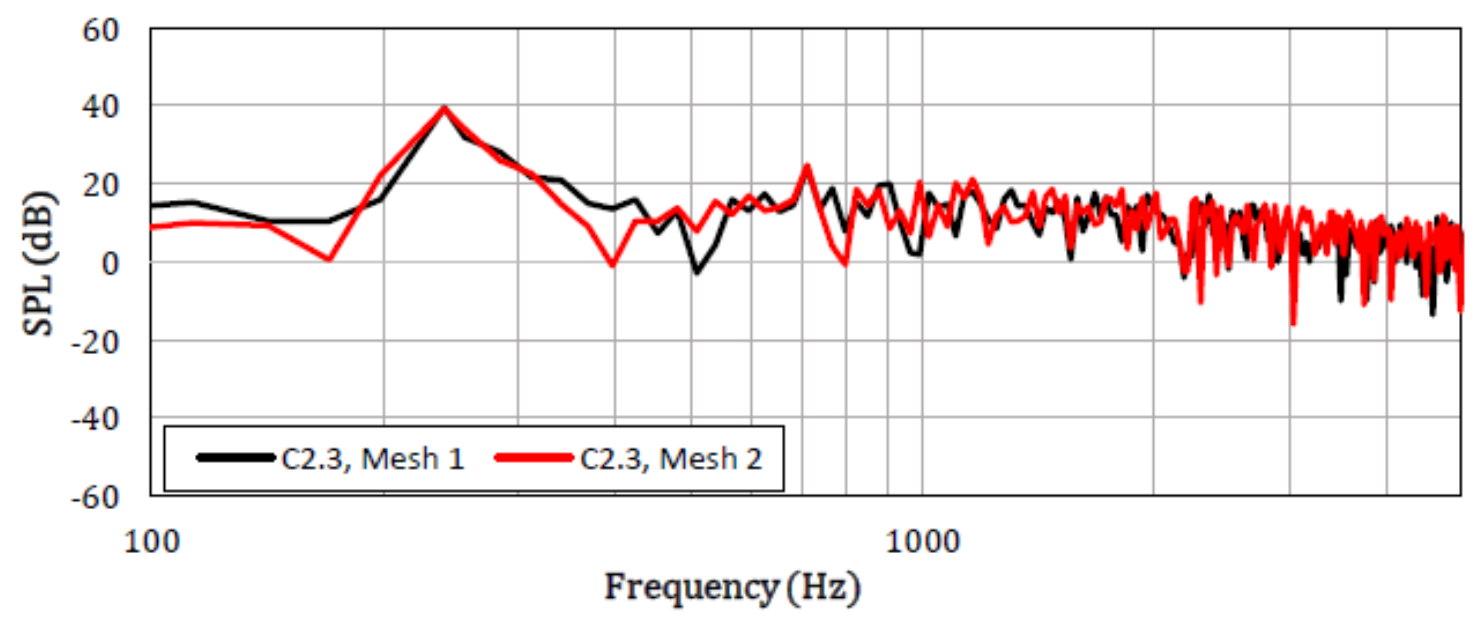

Figure 5.18: Frequency domain noise signals of $\mathrm{C} 2.3$ using 2 meshes.

As part of the current study, and in addition to the numerical predictions, wind tunnel testing has been performed to measure the noise of a NACA-0012 airfoil with a straight TE and a serrated sawtooth TE. The airfoil has a chord length $\mathrm{c}$ of $300 \mathrm{~mm}$, and the width is similar to the width of the nozzle exit at $510 \mathrm{~mm}$. The airfoil AoA is set to zero and fixed to the nozzle exit by two side plates. The microphone was placed at about 1.4 $\mathrm{m}$ from the TE at a polar angle of $90^{\circ}$. The free jet velocity was set to $24 \mathrm{~m} / \mathrm{s}$ and the flow, parameters and chord length are similar to the computational cases, yielding a $R e_{c}$ of approximately 500,000. The fluctuating pressure-time signals for the used microphone are recorded and then used to calculate the SPL spectrum. The data sampling frequency is set to $20 \mathrm{kHz}$ and the data sampling period is 30 seconds, corresponding to a frequency resolution of $0.033 \mathrm{~Hz}$. The obtained signal is also passed through a time-domain filter to remove the low and high frequency contamination, caused by the microphone's low frequency roll off and high-frequency aliasing. The band-pass filter used is a Butterworth filter with the first and second stopband frequencies of 100 and $f_{s} / 2 \mathrm{~Hz}$ respectively, where $f_{s}$ is the sampling frequency. The sound pressure level, SPL, is computed using the root mean square (RMS) of filtered pressure signal using the following equation: 


$$
S P L=10 \log _{10}\left(\frac{P_{R M S}^{2}}{P_{r e f}^{2}}\right)
$$

where $P_{r e f}$ is the standard reference pressure in air, $20 \mu \mathrm{Pa}$. Figure 5.19 shows the turbulent broadband noise spectra (SPL) radiated by a straight TE and a serrated TE, respectively. Note that the serrated TE is a non-flat plate type where a certain degree of bluntness exists at each sawtooth root for all experimental and computational cases. Vortex shedding has been shown to be emanated from the blunt roots, which then proceeds to generate the tonal noise. A tonal peak is observed at $290 \mathrm{~Hz}$ in case E2 and broadband reduction occurs at frequencies higher than the tonal component $(350 \mathrm{~Hz}$ to $5 \mathrm{kHz})$.

The numerical simulations and the wind tunnel experiments predict comparable acoustic behavior; by comparing $\mathrm{C} 1.2$ to $\mathrm{E} 0$ and $\mathrm{C} 2.3$ to $\mathrm{E} 2$, the numerically simulated and experimentally obtained SPL levels follow similar acoustic spectra shapes, but with different amplitude. This is mainly caused by limited computational domain span, compared to the experimental one $\left(\operatorname{span}_{E L E S}=30 \mathrm{~mm}\right.$, $\left.\operatorname{span}_{\text {Experimental }}=500 \mathrm{~mm}\right)$, and the noise source contribution of the airfoil leading edge, which is not accounted for in the used ELES configuration. Since the work is focused on TE noise, it is reasonable to neglect the noise radiated by other sections of the airfoil. Narrowband tonal peaks are predicted in both numerical and experimental far-field spectra for the case of sawtooth serrations having $2 \mathrm{~h}=60 \mathrm{~mm}$ and $\lambda=10 \mathrm{~mm}$ (C2.3 and $\mathrm{E} 2)$. The difference in the predicted tonal frequencies can be attributed to the subtle differences that exist between the numerical and experimental geometric models and inflow conditions, such as turbulence, as well as the intrinsic errors of the computational approximations used to decouple noise generation from its propagation. Sawtooth serrations are experimentally confirmed to reduce the generated broadband noise at the expense of adding a narrow-band tonal peak, caused 
by vortex shedding associated with the bluntness of the serration roots. The broadband reduction was not captured in the numerical simulations. Increasing the sensitivity of the simulation would require a finer mesh and longer simulation time, which would render the simulations unfeasible. For the broadband reduction to be captured without significantly increasing the simulation run-time, more powerful computer clusters are required to allow for a larger LES domain and a higher frequency resolution (longer sampling period). The obtained accuracy is found to be satisfactory for the purposes discussed in this paper, when compared to the corresponding reduction in computing effort. ELES successfully predicted the narrowband tonal noise component at a relatively low computational cost, and was used to assess the effect of different serration parameters on the tonal peak and frequency.

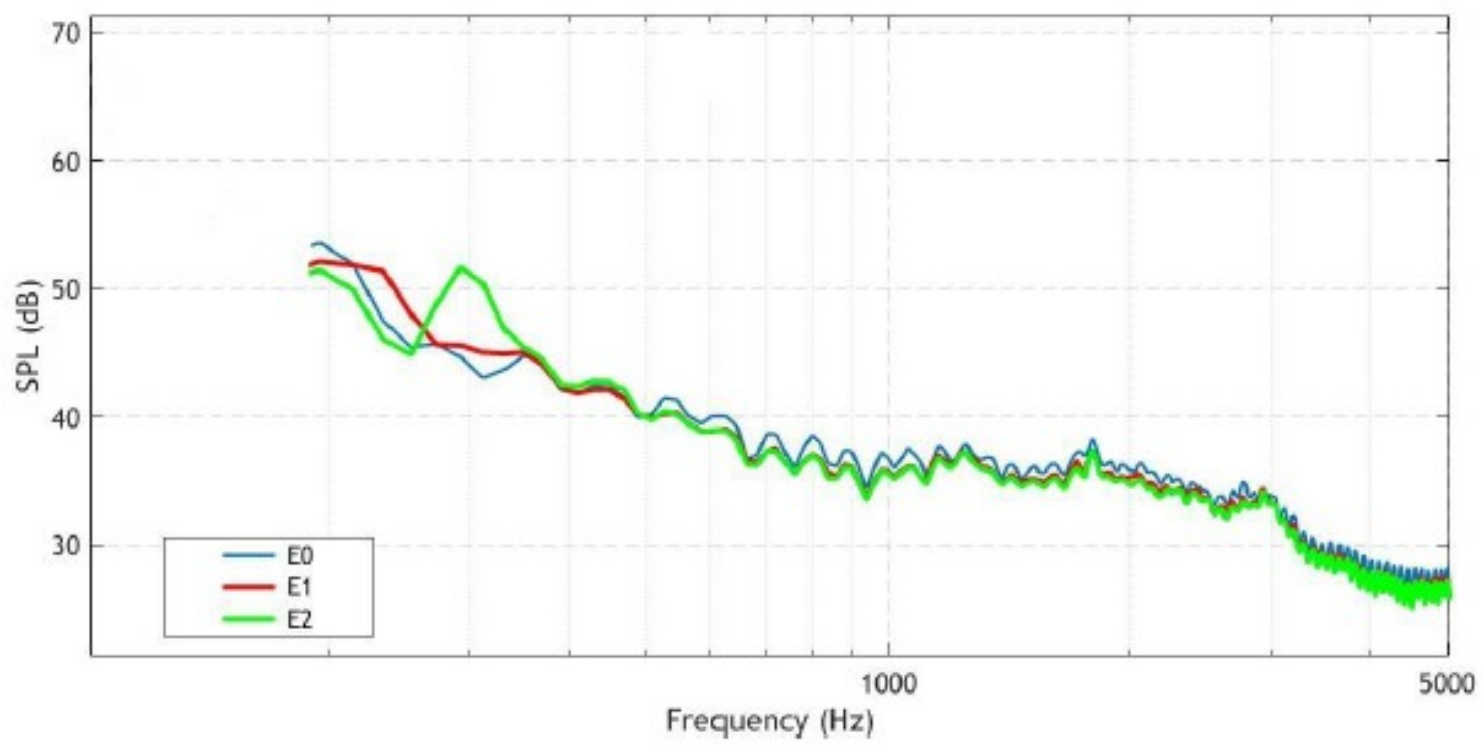

Figure 5.19: Experimentally obtained SPL.

\subsection{Conclusion}

Embedded Large Eddie Simulations as well as experimental wind tunnel testing are 
carried out for NACA-0012 airfoils having different TE configurations. Different sawtooth serrations having various serration amplitudes and wavelengths were investigated for a freestream flow velocity $u_{\infty}=24 \mathrm{~m} / \mathrm{s}, A o A=0^{0}$ and $R e_{c}$ of approximately 500,000. A mesh convergence study is performed and the obtained pressure coefficient distribution is validated. Results show excellent agreement with experimental data and full LES predictions. The validated computational approach is employed to gain an improved understanding of the flow characteristics of serrated-TE airfoils, as well as predict any acoustic tones, while experimental testing is conducted to obtain highly accurate acoustic results.

The introduction of serrations is shown to strongly affect the flow field, mitigating the sharp TE discontinuity and improving mixture between the upper and lower sides of the airfoil. Serrations are shown to concentrate the maximum pressure fluctuation region to downstream of the serration roots. Due to the introduced bluntness of these non-flat plate type sawtooth serrations, vortices are shed from the serration roots, generating narrowband tonal peaks. Narrow-band tonal peaks are observed in the far-field noise spectra at $397 \mathrm{~Hz}, 198 \mathrm{~Hz}$ and $240 \mathrm{~Hz}$ for C2.1, C2.2 and C2.3, respectively. The tonal peak amplitudes are equal for $\mathrm{C} 2.2$ and $\mathrm{C} 2.3$, which share the same $2 \mathrm{~h}=60 \mathrm{~mm}$ and $\varepsilon=16.3 \mathrm{~mm}$. The peak amplitude was lower in $\mathrm{C} 2.1$ by $3.3 \mathrm{~dB}$, suggesting that the tonal noise is louder for cases having longer serrations and increased root bluntness. Longer serrations, and higher values of $\epsilon$ are responsible for the strength of the shed vortices and the intensity of the radiated tonal noise. The frequency at which the tonal peaks occur can be controlled for the same $2 \mathrm{~h}$ and $\varepsilon$ by modifying the wavelength, $\lambda$. Larger values of $\lambda$ for the same $2 \mathrm{~h}$ and $\epsilon$ lead to higher tonal peak frequencies, and larger values of $\varepsilon$ for the same $2 \mathrm{~h}$ and $\lambda$ lead to lower tonal peak frequencies accompanied by higher peak amplitudes. Experimental results confirm that sawtooth serrations reduce the broadband generated noise at the expense of adding a tonal peak. Qualitative comparisons are made between computational results and experimental measurements and satisfactory agreement is achieved. 
The numerical simulations and the wind tunnel experiments predict similar acoustic behavior and shape of the far-field noise spectra. In combination with the FW-H analogy, ELES successfully captures the narrowband peaks of the radiated far-field noise, which are associated with vortex shedding. The results of this investigation illustrate how ELES can be used as a reasonable alternative to the more computationally demanding full LES or direct numerical simulation approach-es. Future work aims to utilize the aforementioned methods for the development of new noise-suppressing TE designs. 
Chapter

\title{
Experimental Investigation of
}

NACA-0012 Airfoil Instability Noise with Sawtooth Trailing Edges

\begin{abstract}
This chapter presents an experimental study of the effect of sawtooth trailing-edge serrations on airfoil instability noise. This work is relevant to reducing the noise from aircraft wings, wind turbines and cooling fans. The far-field noise measurements are obtained to investigate the noise radiation characteristics of a NACA-0012 airfoil operated at various angles of attack: $0^{0}, 5^{0}$, and $10^{0}$, and covered Reynolds numbers of $2.87 \times 10^{5}$, $3.71 \times 10^{5}$, and $5.00 \times 10^{5}$, corresponding to free stream velocities of 14,18 , and $24 \mathrm{~m} / \mathrm{s}$, respectively. It is found that as the Reynolds number increases, the instability noise shifts from tonal to broadband, whereas as the angle of attack increases, it shifts from broadband to tonal. Furthermore, sawtooth trailing-edges are used to minimize instability tone noise, leading to considerable self-noise reduction. Parametric studies of the serration amplitude $2 h$ and streamwise wavelength $\lambda$ are performed to understand the effect of sawtooth
\end{abstract}


trailing-edges on noise reduction. It is observed that the sound pressure reduction level is sensitive to both the amplitude and streamwise wavelength. Overall, the sawtooth trailing-edge with larger amplitude and smaller wavelength produce the greatest amount of noise reduction.

\subsection{Introduction}

Noise emitted from the trailing-edge (TE) of an airfoil is believed to be a major noise source in many industrial applications, such as wind turbines, high lift devices on aircraft airframes, and cooling fan blades. The character and level of trailing-edge self-noise are known to be highly sensitive to Reynolds number (free stream velocity), angle of attack, airfoil geometry, and trailing-edge bluntness [1].

Trailing-edge noise radiated in high Reynolds number flow is typically broadband in nature [56,77]. In contrast, TE noise has a characteristic narrowband structure consisting of a broadband hump superimposed with many tones at low Reynolds numbers with minor residue turbulence in the free stream. The convective turbulent eddies in the boundary layer will scatter efficiently into "broadband noise" at the TE if the chord length of the airfoil is greater than the acoustic wavelength. For Reynolds number of $2.87 \times 10^{5} \leq R e_{c} \leq 5.00 \times 10^{5}$ the boundary layer on the airfoil surface is in transition from laminar to turbulent, which makes the flow unstable. Hydrodynamic instabilities, such as the Tollmien-Schlichting (T-S) waves, build in the boundary layer under specific conditions and finally scatter into noise at the trailing-edge. This mechanism of self-noise is referred to as instability tonal noise 136,137.

Tam 138 claimed that tonal noise was caused by a feedback loop between the oscillat-

ing wake and the trailing-edge of the airfoil. A nominal two-dimensional vortex shedding will form downstream of a blunt TE once a moderate Reynolds number is reached, from 
which narrow-band tone noise will be emitted from the shear layer [53,97]. Studies on a NACA-0012 airfoil have shown that existence of a separation zone at the trailing-edge on the pressure surface is required for a broadband hump and/or tones to occur [139, 140]. It was concluded that, before tonal noise becomes efficiently radiated, the incoming $\mathrm{T}-\mathrm{S}$ waves must be amplified by the separating shear layer. For most symmetrical airfoils, at the back of the airfoil, an adverse pressure gradient always exists, and its magnitude is determined by the profile and angle of attack of the airfoil. These characteristics have an impact on the separation region, which ultimately affects the intensity and frequency of the radiated instability tonal noise.

To date, practically all research on trailing-edge noise in airfoils with serrations has focused on lowering broadband self-noise [46, 48, 141]. As mentioned previously, broadband self-noise is mostly associated with turbulent boundary layers at high Reynolds numbers. On the other hand, the use of sawtooth trailing-edges in an adverse pressure gradient zone has been shown to minimize the tendency for flow separation [76]. The laminar boundary layer at the sawtooth trailing-edge will be more resistant to separation in low Reynolds number flow, which could directly affect the efficiency of the instability tonal noise radiation. As a result, using serrations to manage instability tones has the potential to be effective. It's important to note that the use of serrations to reduce instability self-noise and broadband self-noise happens through different mechanisms. This section is mostly concerned with the former and relates to an airfoil noise research conducted at low to medium Reynolds numbers.

The aim of this study is to investigate if flow separation can be avoided with the application of trailing-edge serrations, resulting in lower tonal noise instability. Because of the serrated trailing-edge's capacity to create turbulent wake flow, this mechanism might effectively eliminate a tonal noise source in the wake. In addition, this work has the objective to establish the relationship between the level of instability self-noise reduction and the serration parameters (in terms of the spanwise wavelength $\lambda$ and the root-to-tip 
length 2h). Results from this experimental investigation are hoped to aid in the design of a low-noise airfoil appropriate for low to moderate Reynolds number flows.

\subsection{Experimental setup}

\subsubsection{Airfoil model and TE serrations}

The airfoil under investigation is a NACA-0012 airfoil with a sawtooth TE serration cut directly into the main body of the airfoil as shown in Figure 6.1. The airfoil model with the straight trailing-edge, Str.TE, is used as the reference configuration for all tests and so will be referred to as the reference. The chord length of the airfoil is $300 \mathrm{~mm}$, and the width is $510 \mathrm{~mm}$. Between the leading-edge $\mathrm{x} / \mathrm{c}=0$ and $\mathrm{x} / \mathrm{c}=0.73$, the original NACA0012 airfoil model profile is unmodified, where $\mathrm{x}$ is the streamwise direction. Further downstream, $0.73 \leq \mathrm{x} / \mathrm{c} \leq 1.0$, is a section that can be removed and replaced by either a straight or modi-fied trailing-edge profile. Once attached, the trailing-edge section forms a continuous profile giving the appearance that the sawtooth serrations are cut into the main body of the NACA-0012 airfoil. Also, boundary layer tripping elements were applied using rough sandpaper near the leading edge on both sides of the airfoil at $\mathrm{x} / \mathrm{c}=0.15$, as later shown in Figure 6.7b. 


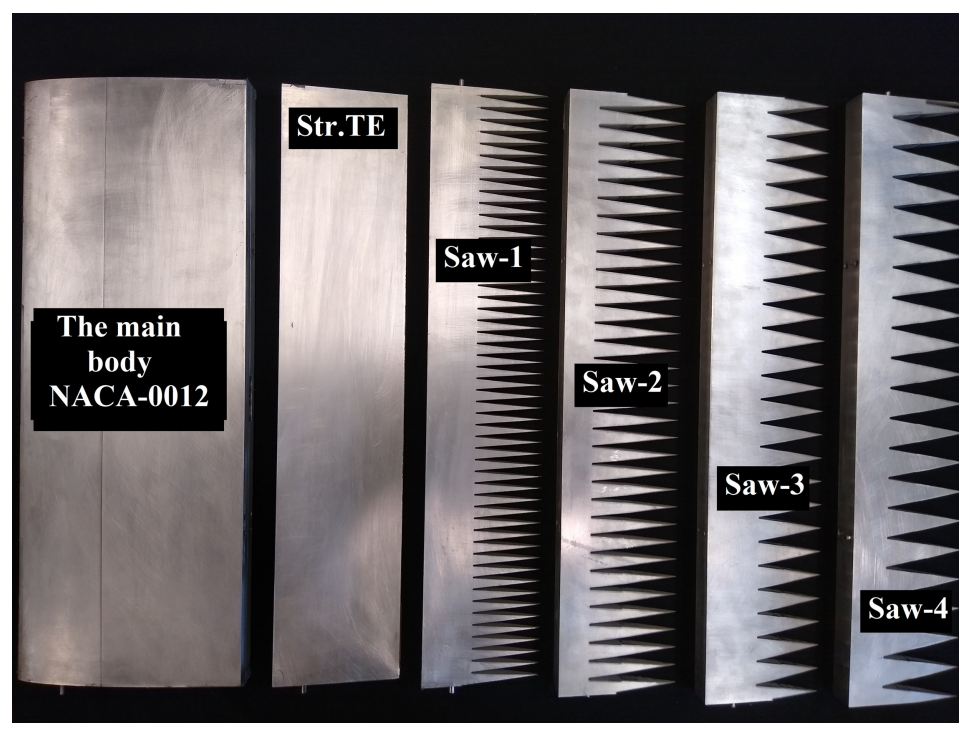

Figure 6.1: Airfoil model and trailing-edges: Straight trailing-edge (Str.TE), and Saw-1, Saw-2, Saw-3 and Saw-4.

Typical parameters associated with an airfoil serrated trailing-edge geometry include the serration amplitude $2 h$ (longitudinal distance from serration tip-to-root), and the serration wavelength $\lambda$ (spanwise distance from sawtooth tip-to-tip). A prominent feature for an airfoil with this type of serrated trailing-edge is that it has significant bluntness $(\varepsilon)$ at the root region. Table 6.1 summarizes the geometrical parameters of the trailing-edge devices manufactured and investigated in this study. Note that from herein all reference to the straight trailing-edge will be represented by Str-TE, and the four sawtooth TEs will be represented by Saw-1, Saw-2, Saw-3 and Saw-4, respectively.

Table 6.1: Design parameters of TE in the present study.

\begin{tabular}{ccccc}
\hline Model & $\mathrm{c}(\mathrm{mm})$ & $2 \mathrm{~h}(\mathrm{~mm})$ & $\lambda(\mathrm{mm})$ & $\varepsilon(\mathrm{mm})$ \\
\hline Str-TE & 300 & - & - & - \\
\hline Saw-1 & 300 & 60 & 10 & 16.6 \\
\hline Saw-2 & 300 & 60 & 20 & 16.6 \\
\hline Saw-3 & 300 & 70 & 25 & 18.2 \\
\hline Saw-4 & 300 & 80 & 25 & 20 \\
\hline
\end{tabular}




\subsubsection{Wind tunnel facility}

The experiments were conducted in a closed loop, low-speed wind tunnel at Carleton University as shown in Figure 6.2. The wind tunnel has rectangular exit cross-section with a height of $0.3 \mathrm{~m}$ and a span of $0.73 \mathrm{~m}$. The maximum velocity in the test section is about $900 \mathrm{rpm}$ and the freestream turbulence intensity is about $0.27 \%$. The airfoil model under was mounted vertically across the entire width of the test section. After taking into account the maximum velocity achievable by the current wind tunnel, a Reynolds number of $2.87 \times 10^{5}$ (freestream velocity of $14 \mathrm{~m} / \mathrm{s}$ ), $3.71 \times 10^{5}$ (freestream velocity of $18 \mathrm{~m} / \mathrm{s}$ ) and $5.00 \times 10^{5}$ (freestream velocity of $24 \mathrm{~m} / \mathrm{s}$ ) were chosen for this study. Further details of the facility can be found in [28,29].

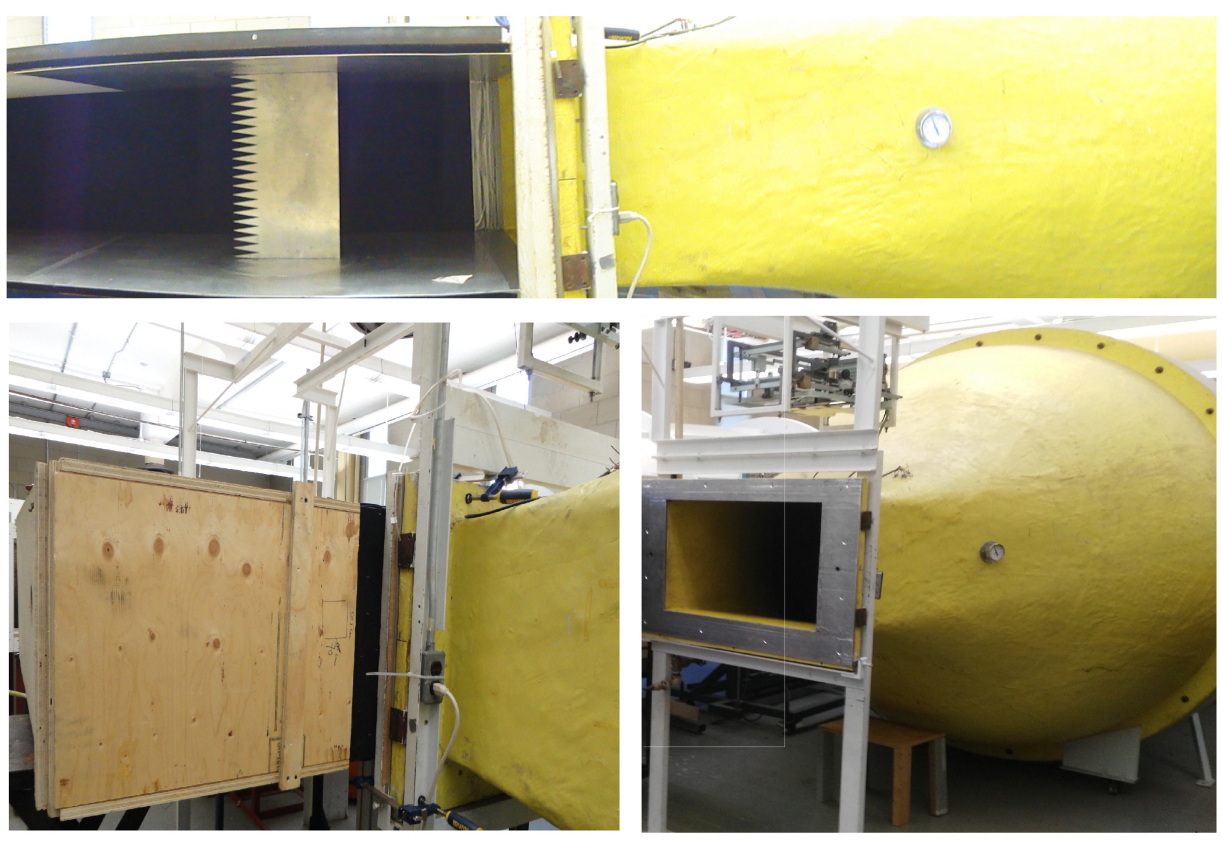

Figure 6.2: Wind tunnel at Carleton University.

\subsubsection{Instruments and procedures}

To measure the radiated self-noise from the airfoil, a single calibrated microphone (Bruel \& Kjaer (B\&K) 4944-A, $\frac{1}{4}$ inch) at angle of $\theta=90^{\circ}$ was used, at a distance of $1.4 \mathrm{~m}$ 


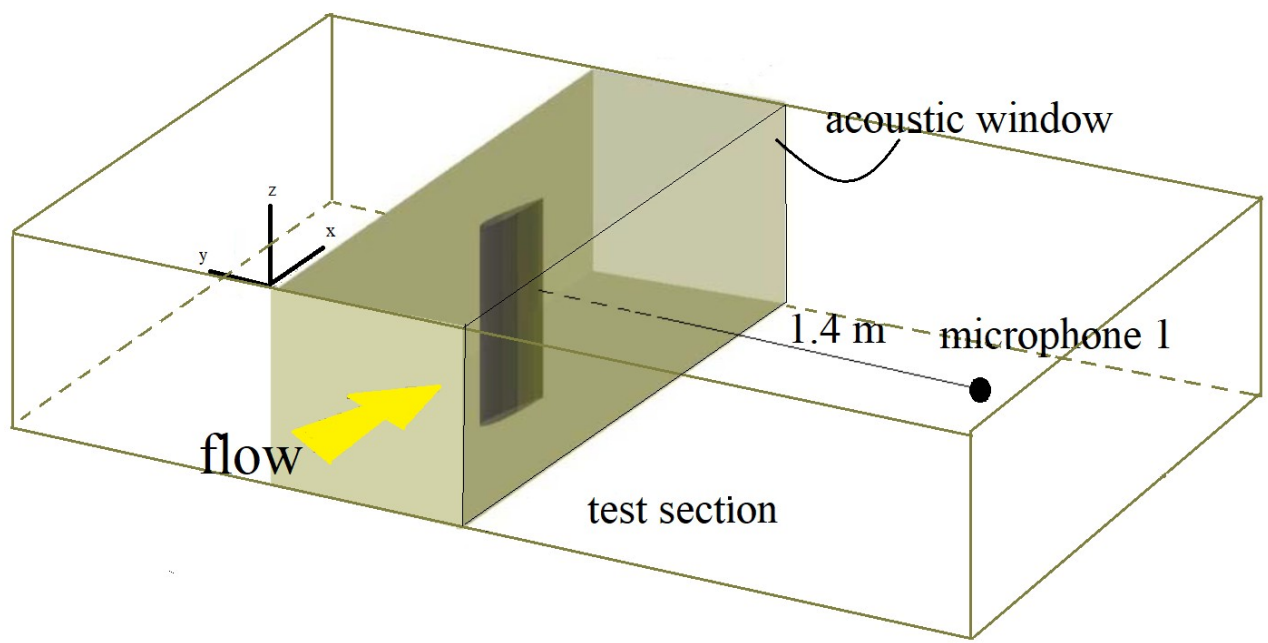

Figure 6.3: Sketch of the experimental setup for the aeroacoustic measurements.

perpendicular from the airfoil trailing-edge and mid-span height as shown in Figure 6.3.

For all acoustic results, the time and the fluctuating pressure measured by the microphone are recorded, and this is data used to obtain the sound pressure level (SPL). Signals from the microphone were amplified by a B\&K Nexus amplifier before digitally stored in a pc through an A/D converter of 24-bit resolution. During the experiment, the acoustic data is sampled at $20 \mathrm{kHz}$ and recorded for 30 seconds. The digitized data was passed through a time domain filter to remove low and high frequency contamination, caused by the microphone's low frequency roll off and high-frequency aliasing. The band-pass filter used is a Butter-worth filter with the first and second stop-band frequencies of 100 and $\mathrm{fs} / 2 \mathrm{~Hz}$, respectively, where fs is the sampling frequency. The attenuation is $60 \mathrm{~dB}$ for both the first and second stop-band. The pass-band ripple is kept as the $1 \mathrm{~dB}$ default and the band match used is stop-band. SPL is computed using the root mean square (RMS) of filtered pressure, as follows:

$$
S P L=10 \log _{10}\left(\frac{p_{R M S}^{2}}{P_{r e f}^{2}}\right)
$$


where $P_{\text {ref }}$ is the standard reference pressure in air, $20 \mu P a$.

The background noise of the facility, i.e., an empty test section without the presence of the airfoil model, was measured prior and after the airfoil noise study. The ranges of flow speed and of angle of attack in which the tonal trailing-edge noise is observed was a key step in the characterization. The first acoustic data was registered by simply listening the sound for determining the limiting conditions of the tonal trailing-edge noise. The measurements were conducted at several velocities (14, 18 and $24 \mathrm{~m} / \mathrm{s})$, corresponding to Reynolds numbers based on the chord of $2.87 \times 10^{5}, 3.71 \times 10^{5}$, and $5.00 \times 10^{5}$, respectively. The clean airfoil exhibits several regimes of tonal noise generation. The registered data were transposed into the $S P L$ versus frequency for different angles of attack and different flow velocities. The investigated angles of attack are $0^{0}, 5^{0}$ and $10^{0}$.

\subsection{Results}

\subsubsection{Occurrence of tones}

Knowing whether tonal noise will be present for a given airfoil, Reynolds number, and angle of attack is essential for a perspective of noise output and signature. In this context, this study starts by verifying the regions of operation in which airfoil tonal noise occur. A NACA-0012 airfoil was earlier studied by Lowson et al. 142. They proposed the involvement of a separated flow in the noise model. According to their model, the shear layer in the laminar separation substantially enhanced the T-S waves. They also indicated a range of conditions (in terms of $R e_{c}$ and angle of attack) where the NACA-0012 airfoil is likely to generate tonal noise. Later, Probsting et al. [6] have compiled results in figure form showing the tonal region and summarizing results from various studies examining different points in and outside of this region. Many experimental observations tend to 
fall in between a bell-shaped envelope (Figure 6.4), as also reported by Desquesnes et al. [143, where tonal noise has often been observed (solid colour symbols).

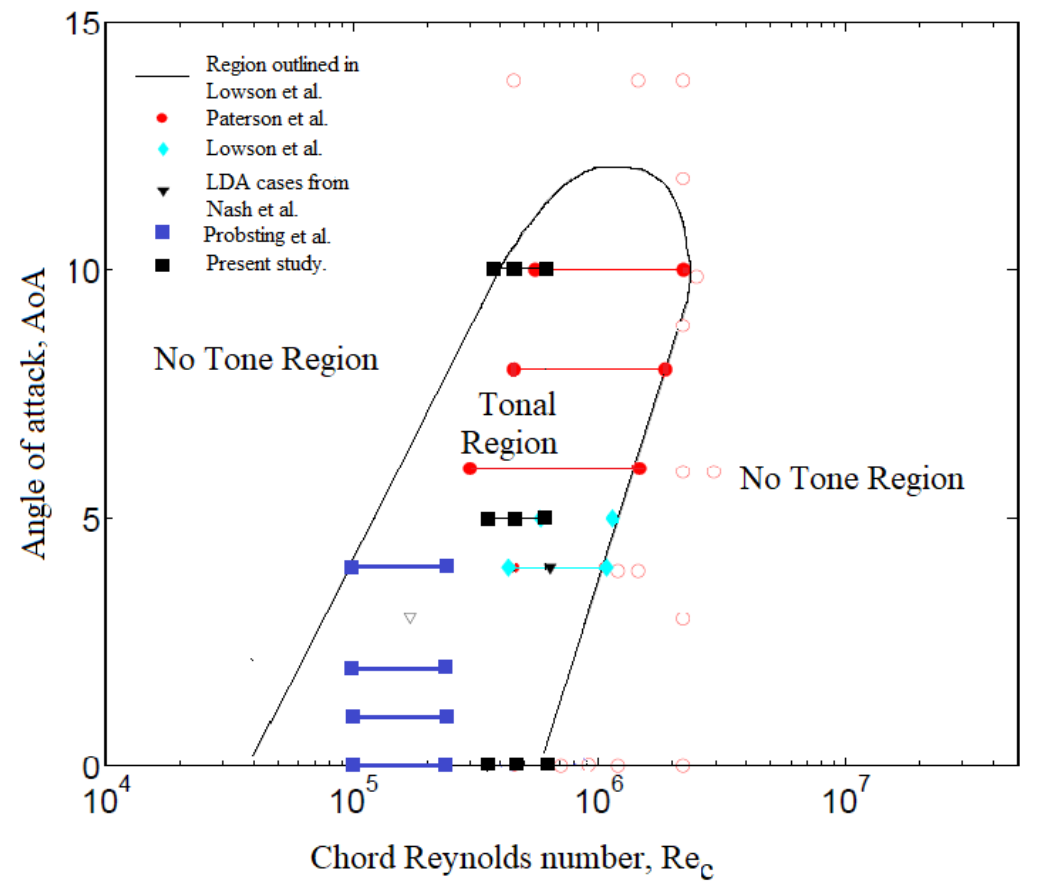

Figure 6.4: Region of Reynolds number, $R e_{c}$, and angle of attack, AoA, where tonal noise can be found for a NACA-0012 Airfoil. Adapted from Probsting et al. [6].

Data from the present study comprises relatively low to moderate Reynolds numbers $2.87 \times 10^{5}-5.00 \times 10^{5}$, and measurement points are represented as black squares in Figure 6.4 and verified within the tonal region. The reduction of tonal noise for lower Reynolds numbers at $\alpha=4^{0}$ is corroborated by the data of Nash et al. 137 and the low-Reynoldsnumber limit of Desquesnes et al. [143. Separation and transition to turbulence tend to occur more upstream on both the suction and pressure sides as the Reynolds number increases, which is believed to be the cause of tonal noise suppression. Instead, the airfoil's acoustic emissions are of a broadband nature in this domain (Paterson et al. [144]). At a 
Reynolds number of approximately $5.00 \times 10^{5}$ for the NACA-0012, this limit is achieved at zero angle of attack; transition will not occur upstream of the trailing-edge.

\subsubsection{Airfoil instability noise with a straight TE}

Figure 6.5 shows the sound pressure level spectra $(S P L)$ radiated by the straight airfoil for three different velocities $(14,18$ and $24 \mathrm{~m} / \mathrm{s})$ at an angle of attack of $0^{0}$. At $14 \mathrm{~m} / \mathrm{s}$ the broadband hump is clearly noticeable with a dominant tone at $351.6 \mathrm{~Hz}$ and followed by two lower tones at $455.8 \mathrm{~Hz}$ and $555.9 \mathrm{~Hz}$, respectively. At $18 \mathrm{~m} / \mathrm{s}$ a single hump is more visible with a marked dominant tone at $545.9 \mathrm{~Hz}$. A remarking point is the disappearance of the tones at $24 \mathrm{~m} / \mathrm{s}$. It is possible that at this combination of flow conditions one of the necessary components for tonal trailing-edge noise such as instability waves, feedback loop or separation bubble is suppressed, hence resulting in the no-appearance of dominate tone. 


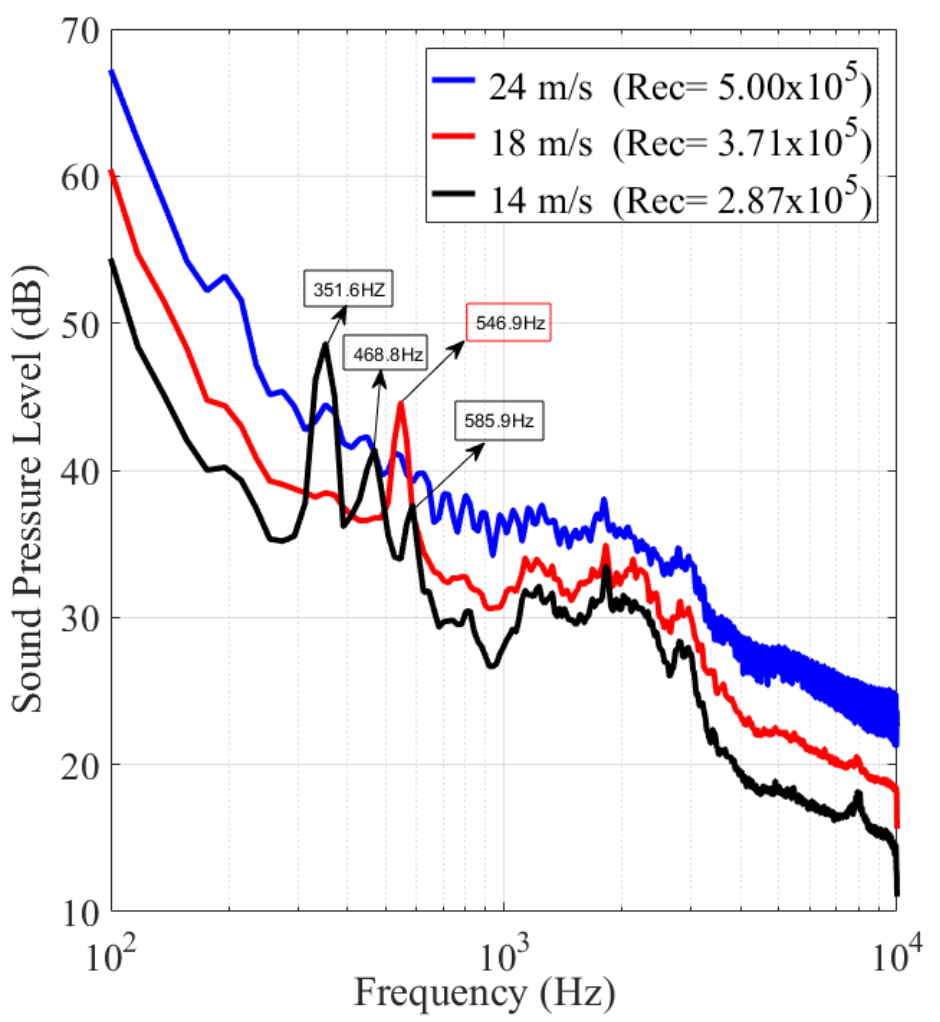

Figure 6.5: $S P L$ spectra radiated by the straight airfoil at $0^{0}$ AoA for various inflow velocities.

It is observed that the tone frequency increases gradually with increasing velocities and tone intensity increases first to a maximum value and then decreases with the velocity. It is also found that the instability noise spectra change from tonal noise to broadband noise with increasing velocities. This change can be attributed to the fact that the flow is now closer to a turbulent Reynolds number $\left(5.00 \times 10^{5}\right)$.

Figure 6.6 shows the sound pressure level spectra $S P L$ radiated by the straight airfoil for various angles of attack at a Reynolds number of $5.00 \times 10^{5}$. It can be observed that there is no distinct tonal noise at $0^{0}$, while the spectrum exhibits three broadband humps at $5^{0}$ between $\sim 300 \mathrm{~Hz}$ to $\sim 600 \mathrm{~Hz}$, and at the largest angle of attack $10^{0}$, the instability noise exhibits an intensive tone at around $449.2 \mathrm{~Hz}$ followed by other lower tones at 527.3 $\mathrm{Hz}$ and $625 \mathrm{~Hz}$. In addition, high harmonic instability noise with much lower sound level is found for angles of attack of $5^{0}$ at $724.2 \mathrm{~Hz}$ and for $10^{0}$ at $898.4 \mathrm{~Hz}$. Overall, the 
instability noise changes from a broadband hump to intensive tonal noise with increasing angle of attack, but the frequency of the main tone does not change significantly with the angle of attack.

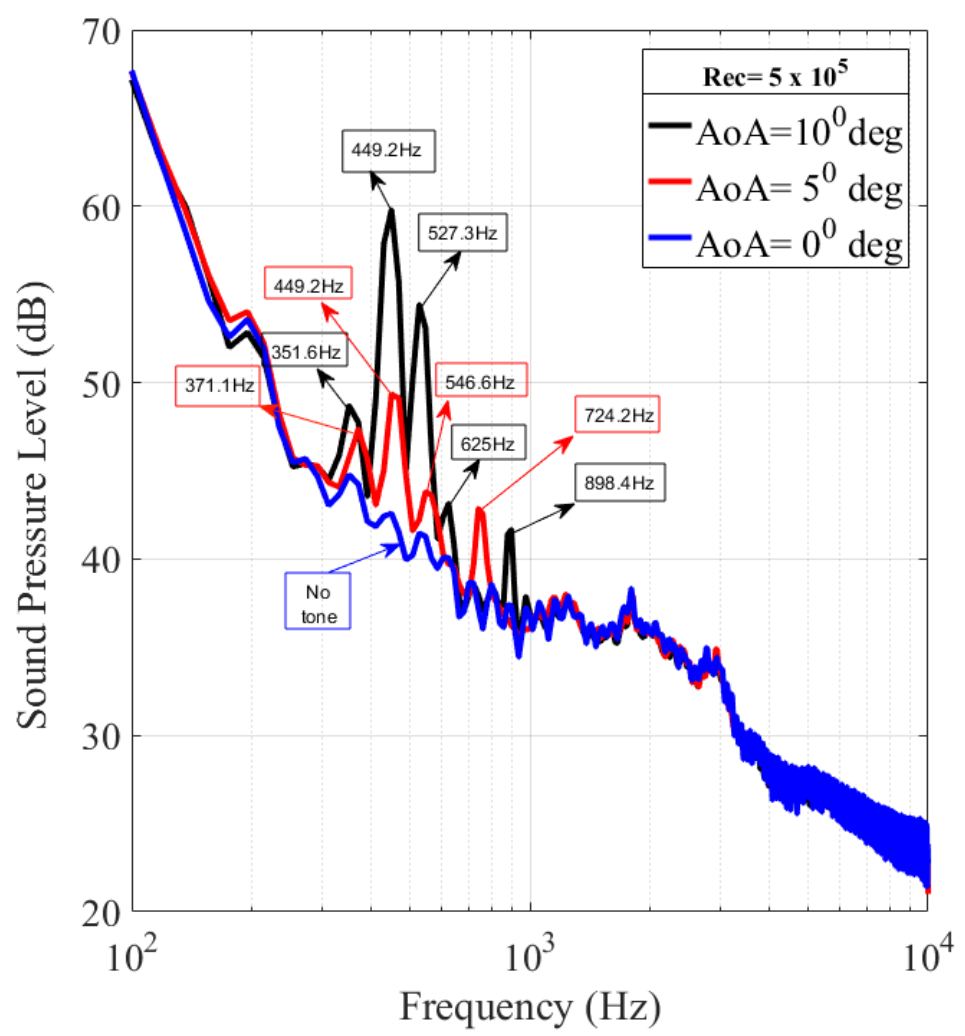

Figure 6.6: $S P L$ spectra radiated by the straight airfoil for various AoA at a Reynolds number of $5.00 \times 10^{5}$.

\subsubsection{Effect of the boundary layer tripping}

Figures $6.7 \mathrm{a}-6.7 \mathrm{~b}$ show the sound pressure level $(S P L)$ spectrum of the airfoil self noise with the straight trailing-edge measured at $\alpha=5^{0}$ AoA for the untripped and tripped cases, respectively, for the various velocities. The spectrum for the untripped case is characterized by tones for different free-stream velocities (Figure 6.7 $)$ ). On the other hand, no noticeable tones are present for the tripped case, and instead broadband self noise is 
the dominant (Figure 6.7b). Boundary layers at both the suction and pressure surfaces are turbulent near the trailing-edge when tripping elements are applied on their respective surfaces. Without tripping, the boundary layer at the pressure surface is laminar (or separated) near the trailing-edge area, and therefore tonal noise occurs.
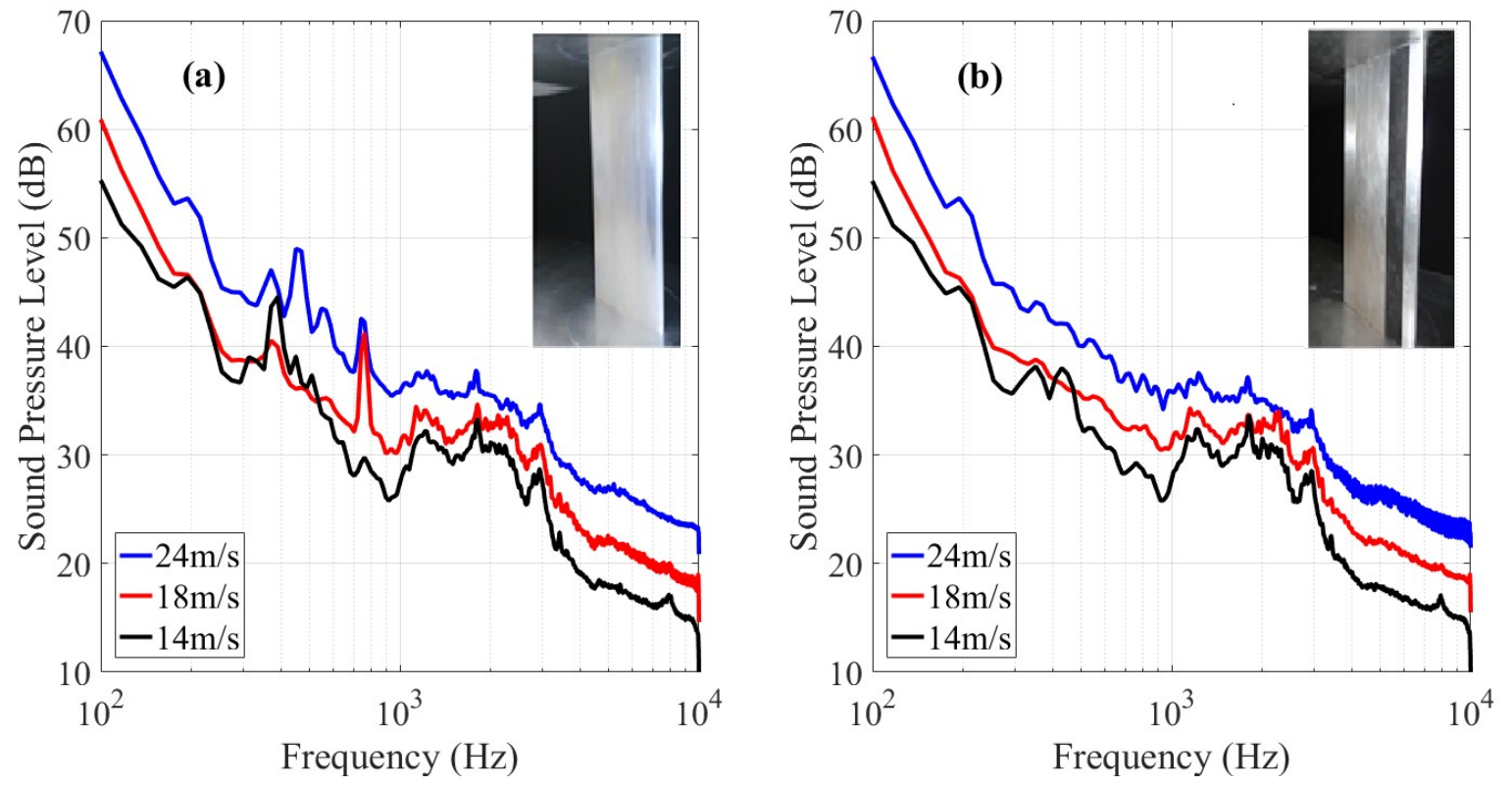

Figure 6.7: $S P L$ measured at $\alpha=5^{0}$ AoA and $\mathrm{U}=14,18$, and $24 \mathrm{~m} / \mathrm{s}$ for; a) untripped airfoil ;b) tripped airfoil.

\subsubsection{Noise reduction by sawtooth TEs}

The effect of sawtooth trailing-edges on airfoil instability noise is investigated and results shown in Figure 6.8. The angle of attack is kept at $0^{0}$ with a Reynolds number of $2.87 \times 10^{5}$ corresponded to the free-stream velocity of $14 \mathrm{~m} / \mathrm{s}$. The sawtooth amplitude $2 h$ ranges from 60 to $80 \mathrm{~mm}$ and the wavelength $\lambda$ varies from 10 to $25 \mathrm{~mm}$, as shown in Table 6.1. It is observed that straight airfoil exhibits intensive instability noise at around $\sim 350 \mathrm{~Hz}$, followed by lower tones at $\sim 470 \mathrm{~Hz}$ and $\sim 570 \mathrm{~Hz}$, respectively, while these tones mechanism are almost completely suppressed by the sawtooth trailing-edges. 
It is noteworthy that even a sawtooth airfoil with small amplitude (Saw-1 and Saw-2) can effectively reduce the instability noise; however, a sawtooth trailing-edge with larger amplitude (Saw-3 and Saw-4) can achieve better noise reduction. A maximum noise reduction level of $\sim 17 \mathrm{~dB}$ is achieved by the Saw-4 airfoil at approximate frequency of $\sim 385 \mathrm{~Hz}$
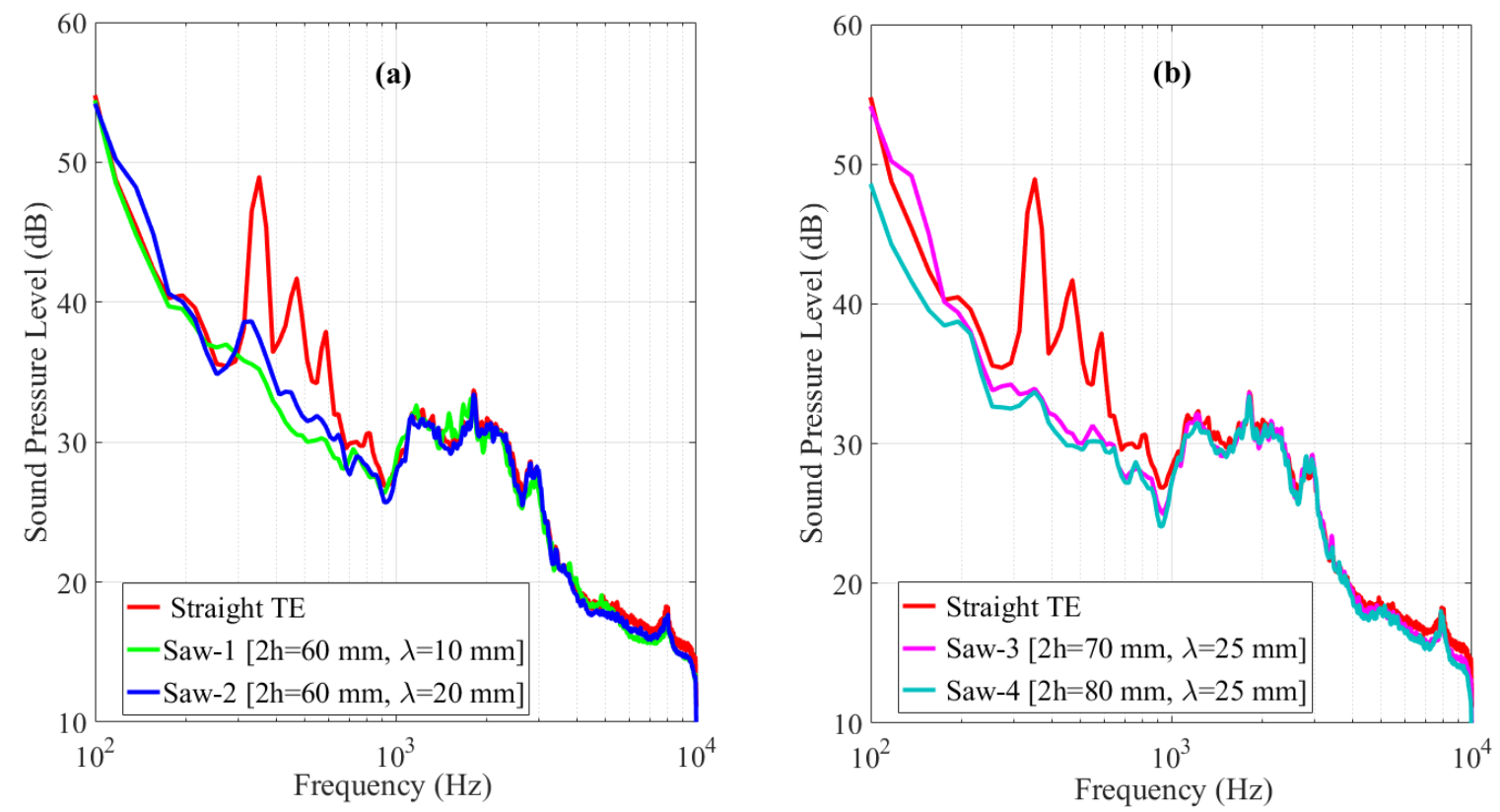

Figure 6.8: Effect of sawtooth trailing-edges on airfoil instability noise at an angle of attack of $0^{0}$ and a Reynolds number of $2.87 \times 10^{5}$; a) Small amplitude (Saw-1 and Saw-2); (b) large amplitude (Saw-3 and Saw-4).

For sawtooth airfoils with larger wavelengths (Saw-3 and Saw-4), the sawtooth trailingedges can also decrease the instability noise, although the noise reduction level of Saw-3 is less than that of the trailing-edge with an amplitude $2 \mathrm{~h}=80 \mathrm{~mm}$ (Saw-4). It is also observed that large serration amplitude is beneficial for noise reduction for larger values of streamwise wavelength $\lambda$, as shown in Figure 6.8(b).

The effect of sawtooth trailing-edges on airfoil instability noise at an angle of attack of $5^{0}$ is depicted in Figure 6.9. The corresponding Reynolds numbers are $3.71 \times 10^{5}$ and $5.00 \times 10^{5}$, respectively. The sawtooth Saw-1 and Saw-2 amplitudes are fixed as $2 \mathrm{~h}=60$ $\mathrm{mm}$ and the wave-length are $\lambda=10 \mathrm{~mm}$ for Saw-1 and $\lambda=20 \mathrm{~mm}$ for Saw-2. As shown 
in Figure 6.9(a), when the Reynolds number is $3.71 \times 10^{5}$, the straight airfoil exhibits intensive instability tonal noise at around $\sim 800 \mathrm{~Hz}$. When the Reynolds number is $5.00 \times 10^{5}$, the straight airfoil exhibits intensive four tonal noise peaks between $\sim 400 \mathrm{~Hz}$ and $\sim 700 \mathrm{~Hz}$.
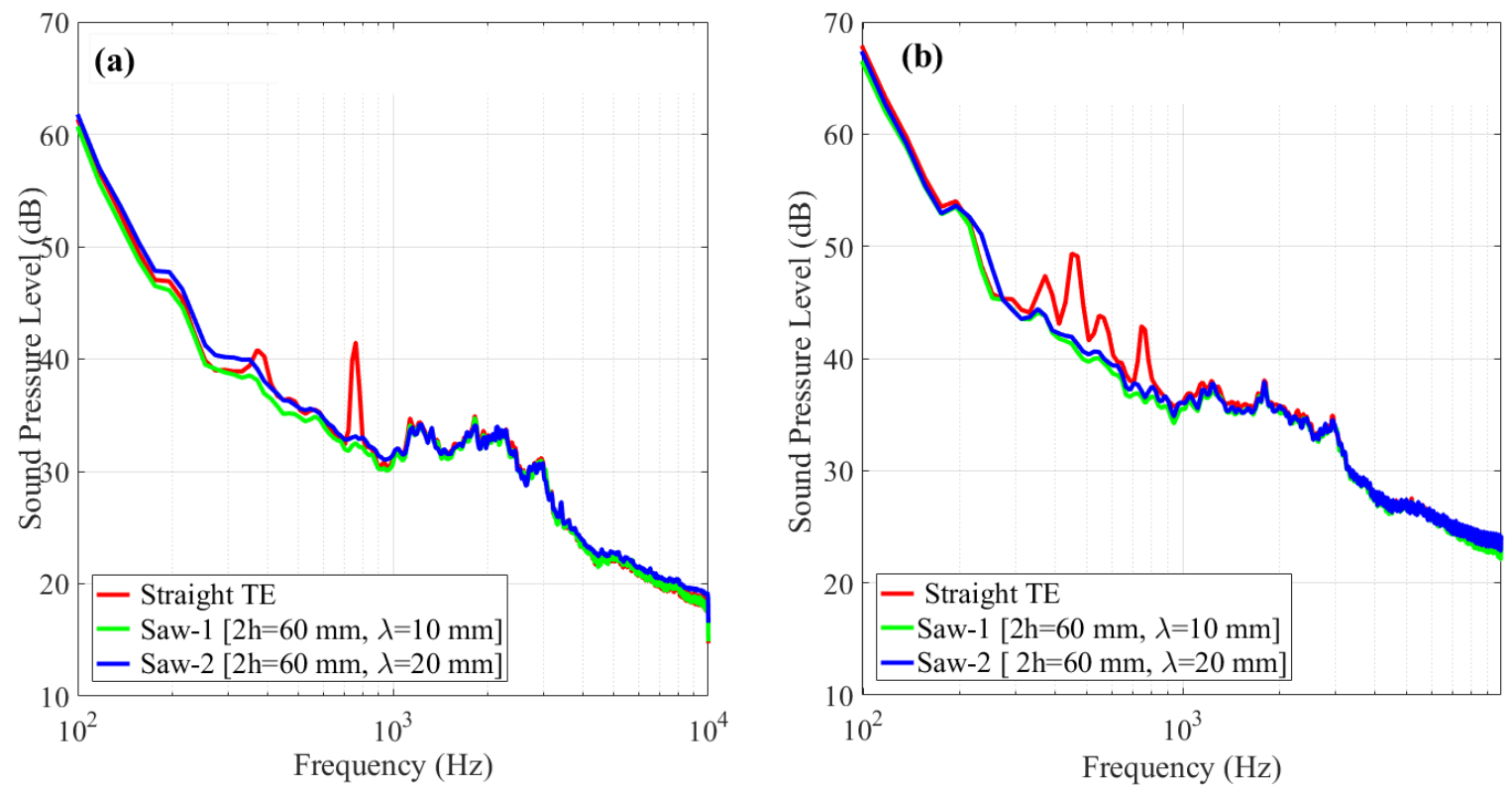

Figure 6.9: Effect of sawtooth trailing-edges on airfoil instability noise at an angle of attack of $5^{0}$; a) $R e_{c}=3.71 \times 10^{5}$; b) $R e_{c}=5.00 \times 10^{5}$.

For the Saw-1 and Saw-2 sawtooth airfoils, the instability noise is eliminated, and the sound spectra exhibit broadband noise. The intensive instability noise disappears for the sawtooth airfoils as shown in Figure 6.9. Maximum noise reduction levels of $\sim 9 \mathrm{~dB}$ at $800 \mathrm{~Hz}$ and $\sim 8 \mathrm{~dB}$ at $550 \mathrm{~Hz}$ is obtained by the Saw- 1 airfoil at Reynolds numbers of $3.71 \times 10^{5}$ and $5.00 \times 10^{5}$, respectively.

The noise reduction effect of sawtooth trailing-edges at an angle of attack of $10^{0}$ is plotted in Figure 6.10 with Reynolds numbers of $3.71 \times 10^{5}$ and $5.00 \times 10^{5}$. At a Reynolds number of $3.71 \times 10^{5}$, the straight airfoil radiates intensive instability tonal noise at around $300 \mathrm{~Hz}$ followed by lower tones at $450 \mathrm{~Hz}$ and $550 \mathrm{~Hz}$, respectively. The Saw- 1 and Saw2 airfoil radiates broadband noise without distinct instability noise. A maximum noise reduction level of $\sim 12 \mathrm{~dB}$ is achieved by the Saw- 1 airfoil at around $\sim 310 \mathrm{~Hz}$. At a 
Reynolds number of $5.00 \times 10^{5}$, the straight airfoil radiates instability noise at around $355 \mathrm{~Hz}, 450 \mathrm{~Hz}, 550 \mathrm{~Hz}$, and $800 \mathrm{~Hz}$. A maximum noise reduction level of $\sim 18 \mathrm{~dB}$ is achieved by the Saw-1 airfoil at of $\sim 490 \mathrm{~Hz}$.
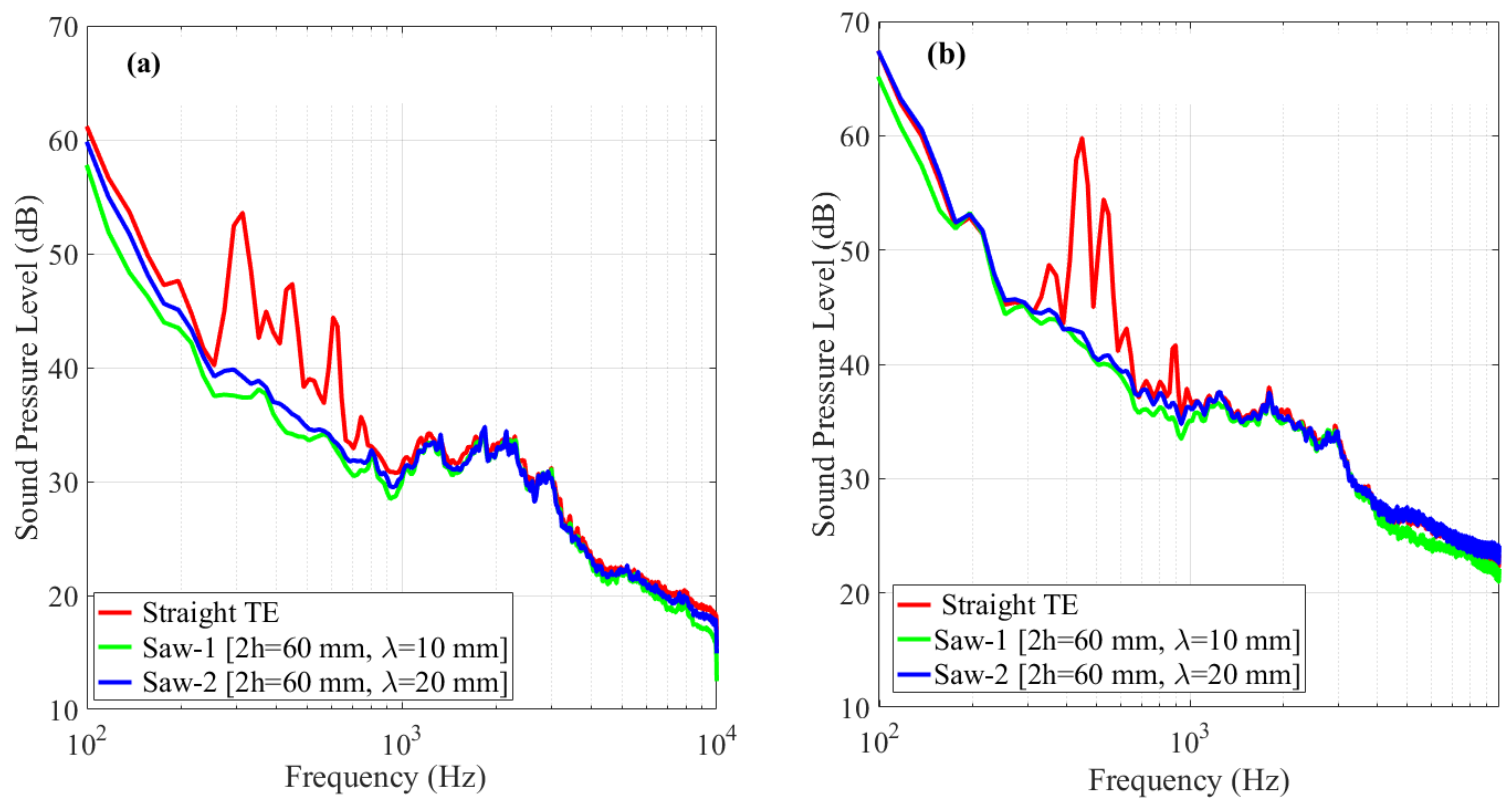

Figure 6.10: Effect of sawtooth trailing-edges on airfoil instability noise at an angle of attack of $10^{0}$ and; a) $R e_{c}=3.71 \times 10^{5}$; b) $R e_{c}=5.00 \times 10^{5}$.

Figure 6.11 further shows the effect of sawtooth trailing-edges on airfoil instability noise at an angle of $10^{0}$ with a Reynolds number of $5.00 \times 10^{5}$, for Saw-3 and Saw- 4 . The instability noise is significantly reduced by the two sawtooth airfoils compared to the straight TE. A maximum noise reduction level of $\sim 17 \mathrm{~dB}$ is obtained at frequency of $\sim$ $490 \mathrm{~Hz}$ by the Saw-4 airfoil. It is notable that broadband noise becomes dominant at frequencies higher than $1000 \mathrm{~Hz}$ for all cases. 


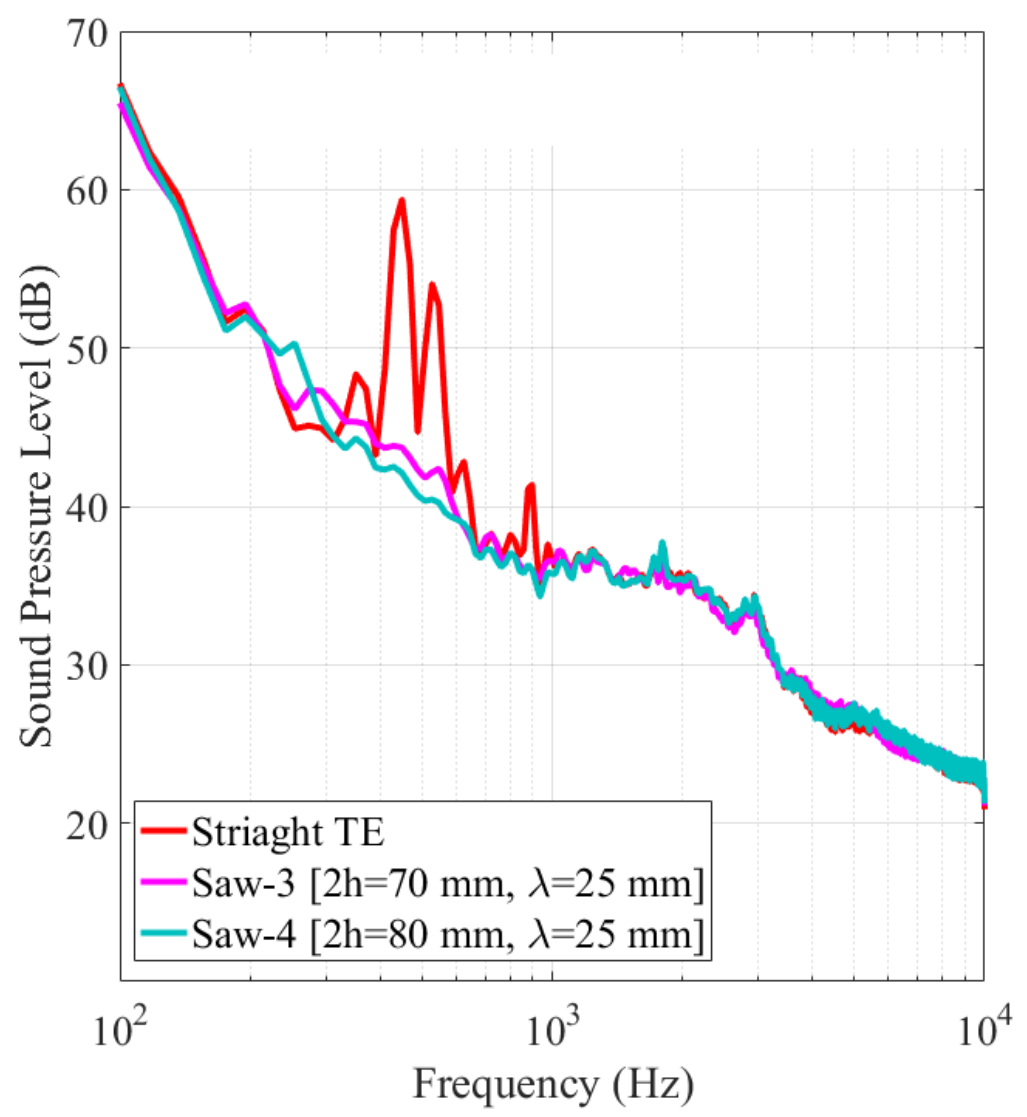

Figure 6.11: Effect of sawtooth trailing-edges on airfoil instability noise at an angle of attack of $10^{0}$ and a Reynolds number of $5.00 \times 10^{5}$.

From Figures 6.8 through 6.11, one can observe that the sawtooth trailing-edge with a larger amplitude and smaller wavelength is generally more effective at reducing instability tonal noise. On a previous study by the authors [28], different TE designs were tested to study airfoil turbulence interaction noise reduction. From optimization and experimental tests conducted by the authors to investigate noise reduction from serrated TEs, the largest noise reduction tends be associated with the sawtooth trailing-edge of the largest amplitude and smallest wavelength. Previously, Howe's work [3,35] has esulted similar conclusions, in which a maximum noise reduction of $\sim 17 \mathrm{~dB}$ was achieved. However, the noise reduction mechanisms of the sawtooth trailing-edges on the airfoil instability noise and airfoil turbulence interaction noise are significantly different. The noise reduction mechanisms on the instability noise are mainly attributed to the strong streamwise 
vorticities generated by the subsidence which can suppress the laminar flow separation near the trailing-edge [101], while the noise reduction mechanisms on the turbulent inflow noise are mainly related to the source cut-off effect and phase interference effect of the sawtooth geometry 3 .

\subsection{Conclusion}

An experimental study has been conducted to investigate the noise radiation characteristics of a NACA-0012 airfoil operated at various angles of attack and low to moderate Reynolds numbers, with different TE configurations. The occurrence of instability noise was observed to be sensitive to both the Reynolds number and angle of attack. The tone frequency increased with increasing velocity, while it did not change significantly with the angle of attack. Various wavelengths and amplitudes of sawtooth trailing-edges were examined in order to suppress instability noise. This investigation has shown that sawtooth trailing-edges can successfully be used to eliminate instability noise, and that sound pressure level reductions were sensitive to both the wavelength and amplitude. The sawtooth trailing-edge with a large amplitude and small wavelength appeared to be the optimum choice to reduce the instability noise. 
Chapter 7

\section{Conclusions and Recommendations for}

\section{Future Work}

Airfoil self-noise is produced when a turbulent boundary layer, TBL, generated on an airfoil surface is scattered by the airfoil's TE. The findings can potentially benefit applications where TE noise is a dominant noise source, such as, aircraft wings and wind turbine blades. The aforementioned is inspired by the owl's silent flight due to its unique wing structure. In order to study the concept of minimizing noise effects, an anechoic wind tunnel section was developed at Carleton University. The test-section walls were treated to simulate an acoustically far-field environment with forwarding flight. Aeroacostic measurements show that the background noise can be comparable with other aeroacoustic wind tunnels worldwide. A simplified airfoil and flat-plate TE noise are tested as a benchmark test. Results show that the serration geometry is effective in reducing noise and the noise radiated from the TE is at least $6 \mathrm{~dB}$ higher than the background noise, satisfying the requirements for aeroacoustic measurements.

The ability of the TE serrations to reduce TBL-TE noise is examined through a numerical optimization study and TEs wind tunnel testing. The noise spectra was initially modeled using semi-empirical models by Howe, for a semi-infinite flat plate, at zero angle of attack and at low Mach numbers. Three different serration geometries are optimized for 
the overall noise from frequency rang of $0.1 \mathrm{kHz}$ to $10 \mathrm{kHz}$. Noise radiation from optimized TEs with different serrations have been investigated. It has been shown that the TE serration design can have a significant effect on the level of noise reduction. Numerical and experimental results show that optimized serrated TE configurations can yield less TBLTE noise compared to the traditional straight TE configuration. Experimental results confirmed that numerical optimization predicted a larger noise reduction compared to measured values.

Noise radiation from optimized trailing-edges with four different sawtooth serrations have been investigated. The noise spectra was modeled using semi-empirical models by Lyu, for a semi-infinite flat plate, at zero angle of attack and at low Mach numbers. It has been shown that the sawtooth TE serration design can have a significant effect on the level of noise reduction. The optimum single size of sawtooth tends to maximum amplitude $h_{\text {opt }}=h_{\max }$, and minimum wavelength, $\lambda_{\text {opt }}=\lambda_{\min }$. Moreover, the greatest noise reduction of up to $12.89 \mathrm{~dB}$ over a wider range of frequencies was conducted by Saw1. Numerical and experimental results are in agreement that optimized serrated trailingedge configurations can yield less TBL-TE noise compared to the traditional straight trailing-edge configuration. The optimization studies underestimated the measurements by about 2 to $12 \mathrm{~dB}$, but the tendency between the different serration geometries was predicted correctly.

The serrated trailing-edge optimization framework for noise reduction, and the studies produced from it in this thesis have contributed to advancements in TBL-TE noise reduction research. The general conclusions of this thesis are presented as follows: 


\subsection{Conclusions}

\subsubsection{Optimization and Testing of Flat-Plate Trailing-Edge Ser- ration Geometry for Reducing Airfoil Self-Noise}

An aeroacoustic wind tunnel test section has been built at Carleton University. The tunnel has a rectangular test section with dimensions of $0.78 \mathrm{~m}$ in width, $0.51 \mathrm{~m}$ in height and $1.83 \mathrm{~m}$ long. Its aeroacoustic performance is measured. Results show that the background noise can be comparable with other aeroacoustic wind tunnels worldwide. A simplified airfoil and flat-plate TE noise are tested as a benchmark test. Results show that the serration geometry is effective in reducing noise and, the noise radiated from the TE is at least $10 \mathrm{~dB}$ higher than the background noise, satisfying the requirements for aeroacoustic measurements.

Three different geometrical profiles of trailing edge serrations have been optimized for the reduction of TBL-TE noise. The optimum width for the three, sawtooth, slitted and sinusoidal serrations, are the smallest allowable one in the overall spectrum frequencies. The optimum single-size sawtooth and sinusoidal always has the largest allowable amplitude whereas the optimum single-size slit has a specific amplitude value to optimize the balance.

Noise radiation from optimized trailing edges with different serrations, sawtooth, slitted and sinusoidal, has been investigated. It has been shown that the TE serration design can have a significant effect on the level of noise reduction. It has been shown that numerical and experimental results are in agreement showing that optimized serrated trailing edge configurations can yield less TBL-TE noise compared to the traditional straight trailing-edge configuration. Moreover, based on the optimization and experimental analyses, the sawtooth serration provides the greatest noise reduction over a wider range of frequencies when compared to slitted and sinusoidal TE serrations. 


\subsubsection{Aeroacoustic Design Optimization and Experimental Inves- tigation of Sawtooth Trailing-Edges for Maximized Noise Reduction}

Noise radiation from optimized trailing edges with different sawtooth serrations have been investigated. It has been shown that the sawtooth TE serration design can have a significant effect on the level of noise reduction. Four different sawtooth geometrical profiles of trailing edge serrations have been optimized for the reduction of TBL-TE noise. The optimum size of sawtooth tends to maximum amplitude and minimum wavelength. Moreover, based on the optimization analyses, the Saw-1 serration provides the greatest noise reduction of up to $12.89 \mathrm{~dB}$ over a wider range of frequencies. It is concluded that numerical and experimental results agree that optimized serrated trailing edge configurations can yield less TBL-TE noise compared to the traditional straight-trailing edge configuration. The optimization studies underestimated the measurements by about $\sim(2$ to 12$) \mathrm{dB}$, but the tendency between the different serration geometries was predicted correctly.

The qualitative and quantitative deviation from experimental results clearly indicates the limitation of adopting the frozen turbulence assumption. A modified model that includes the growth/decay in the turbulent wall pressure fluctuation along the serration shows a better qualitative agreement with the experimental observation. Nevertheless, there is still a large room to improve the accuracy of the noise prediction model for trailing edge serrations, and the development of boundary layers along the serrations should be included in the noise prediction model before it can be applied for optimization purposes.

This work is relevant to reducing the noise from aircraft wings, wind turbines and cooling fans. The results obtained in the current study can serve as a practical approach for more elaborate engineering development of the TE serrations. The optimization problem solved in this study is scalable for various types of constraints on the design space. 
It can be successfully implemented to solve a multi-objective optimization problem which includes aerodynamic and/or structural objectives. Also, the trends predicted in the numerical optimization section are well supported by the experiment. The current study focuses on the sawtooth trailing-edge noise and its reduction. However, the noise reduction mechanism is known to be similar to that for the turbulent boundary layer leading-edge noise. Hence, the present numerical optimization analysis and the optimum proposed sawtooth profile are expected to work in a similar manner for the leading-edge problem. This forms part of our future work.

\subsubsection{ELES and Tonal Noise Prediction of Serrated TE Airfoils}

Embedded Large Eddie Simulations as well as experimental wind tunnel testing are carried out for NACA-0012 airfoils having different TE configurations. Different sawtooth serrations having various serration amplitudes and wavelengths were investigated for a freestream flow velocity $u_{\infty}=24 \mathrm{~m} / \mathrm{s}, A o A=0$ and $R e_{c}$ of approximately 500,000. A mesh convergence study is performed and the obtained pressure coefficient distribution is validated. Results show excellent agreement with experimental data and full LES predictions. The validated computational approach is employed to gain an improved understanding of the flow characteristics of serrated-TE airfoils, as well as predict any acoustic tones, while experimental testing is conducted to obtain highly accurate acoustic results.

The introduction of serrations is shown to strongly affect the flow field, mitigating the sharp TE discontinuity and improving mixture between the upper and lower sides of the airfoil. Serrations are shown to concentrate the maximum pressure fluctuation region to downstream of the serration roots. Due to the introduced bluntness of these non-flat plate type sawtooth serrations, vortices are shed from the serration roots, generating narrowband tonal peaks. Narrow-band tonal peaks are observed in the far-field noise spectra at $397 \mathrm{~Hz}, 198 \mathrm{~Hz}$ and $240 \mathrm{~Hz}$ for C2.1, C2.2 and C2.3, respectively. The tonal peak ampli- 
tudes are equal for $\mathrm{C} 2.2$ and $\mathrm{C} 2.3$, which share the same $2 \mathrm{~h}=60 \mathrm{~mm}$ and $\varepsilon=16.3 \mathrm{~mm}$. The peak amplitude was lower in $\mathrm{C} 2.1$ by $3.3 \mathrm{~dB}$, suggesting that the tonal noise is louder for cases having longer serrations and increased root bluntness. Longer serrations, and higher values of $\epsilon$ are responsible for the strength of the shed vortices and the intensity of the radiated tonal noise. The frequency at which the tonal peaks occur can be controlled for the same $2 \mathrm{~h}$ and $\varepsilon$ by modifying the wavelength, $\lambda$. Larger values of $\lambda$ for the same $2 \mathrm{~h}$ and $\epsilon$ lead to higher tonal peak frequencies, and larger values of $\varepsilon$ for the same $2 \mathrm{~h}$ and $\lambda$ lead to lower tonal peak frequencies accompanied by higher peak amplitudes. Experimental results confirm that sawtooth serrations reduce the broadband generated noise at the expense of adding a tonal peak. Qualitative comparisons are made between computational results and experimental measurements and satisfactory agreement is achieved. The numerical simulations and the wind tunnel experiments predict similar acoustic behavior and shape of the far-field noise spectra. In combination with the FW-H analogy, ELES successfully captures the narrowband peaks of the radiated far-field noise, which are associated with vortex shedding. The results of this investigation illustrate how ELES can be used as a reasonable alternative to the more computationally demanding full LES or direct numerical simulation approach-es. Future work aims to utilize the aforementioned methods for the development of new noise-suppressing TE designs.

\subsubsection{Experimental Investigation of NACA-0012 Airfoil Instabil- ity Noise with Sawtooth Trailing-Edges}

Experimental studies have been conducted to investigate the noise radiation characteristics of a NACA-0012 airfoil operated at various angles of attack and low to moderate Reynolds numbers. The occurrence of instability noise was observed to be sensitive to both the Reynolds number and angle of attack. The tone frequency increased with increasing velocity, while it did not change a lot with the angle of attack. The various 
wavelengths and amplitudes for sawtooth trailing edges were used for the suppression of the instability noise. The influences of wavelength and amplitude on noise reduction were studied. It was found that the sawtooth trailing edges could totally eliminate the instability noise, and the sound pressure reduction levels were sensitive to both the wavelength and amplitude. The sawtooth trailing edge with a large amplitude and small wavelength appeared to be the optimum choice to reduce the instability noise.

\subsection{Future Work}

The work included in this thesis has highlighted a number of new intriguing avenues that can be explored in future work. Potential extensions of this work follow two paths: (1) Numerical Analysis and Optimization, and (2) Experimental Studies. Specific examples of extension topics for each path are outlined below.

\subsubsection{Numerical Analysis and Optimization}

1. Multi-objective optimization studies that consider additional factors to noise reduction. In order to ensure industry acceptance of this technology, other, non-acoustic, factors can be considered. The general considerations made here can be quantified and modeled in terms of engineering specifications, and can then be converted into objective functions. Examples include aerodynamic and/or structural objectives, etc.

2. The optimization method for the sawtooth serrated trailing-edge using Lyu's firstorder solution $\Theta_{n}^{(1)}$ runs for several hours. The results are promising and a future step would be to implement the second-order solution $\Theta_{n}^{(2)}$. Simplifying the model 
is recommended in order to reduce computational costs and time.

3. The current optimization of trailing-edge serrations is only valid for uniform geometry serrations. A next step would involve the optimization for non uniform geometry serrations or for cases were the serration geometry changes across the wing span.

\subsubsection{Experimental Studies}

There are several recommendations for future aeroacoustics tests in the medium speed wind tunnel at Carleton University, as following:

1. The major source of low-frequency noise that may require attention is the vibration and tonal noise from the motor, turning vanes, and fan, so balancing and greasing is recommended to sufficiently reduce vibrations and eliminate the tonal noise.

2. The background levels are competitive, but further reduction is desired. Noise reduction was recently achieved by replacing the controller (VDC) (as shown in Figure 7.1).

3. Additional acoustic treatment can be added to the wind tunnel facility (e.g. inlet silencer, isolation around the motor, vibration isolation frames, etc.). 


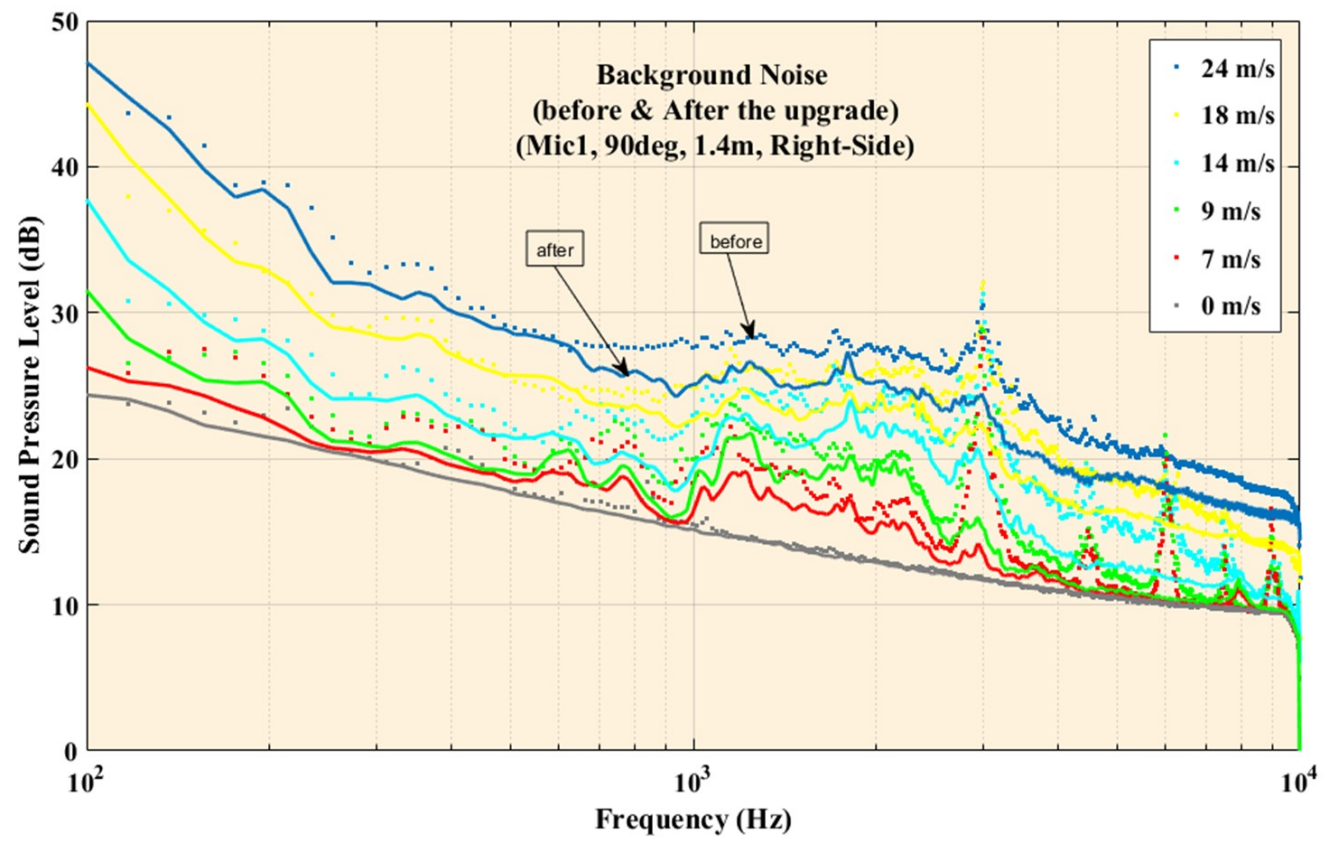

Figure 7.1: Wind tunnel upgrading. 


\section{References}

[1] T. F. Brooks, D. S. Pope, and M. A. Marcolini, "Airfoil self-noise and prediction," 1989.

[2] T. Bachmann, H. Wagner, and C. Tropea, "Inner vane fringes of barn owl feathers reconsidered: morphometric data and functional aspects," Journal of anatomy, vol. 221, no. 1, pp. 1-8, 2012.

[3] M. S. Howe, "Noise produced by a sawtooth trailing edge," The Journal of the Acoustical Society of America, vol. 90, no. 1, pp. 482-487, 1991.

[4] M. Azarpeyvand, M. Gruber, and P. Joseph, "An analytical investigation of trailing edge noise reduction using novel serrations," in 19th AIAA/CEAS aeroacoustics conference, 2013, p. 2009.

[5] B. Lyu, M. Azarpeyvand, and S. Sinayoko, "A trailing-edge noise model for serrated edges," in 21st AIAA/CEAS Aeroacoustics Conference, 2015, p. 2362.

[6] S. Pröbsting, J. Serpieri, and F. Scarano, "Experimental investigation of aerofoil tonal noise generation," Journal of Fluid Mechanics, vol. 747, p. 656, 2014.

[7] M. Lighthill, "On sound generated aerodynamically, proceedings of the royal society of london," Series A, Math Phys Sci, vol. 211, pp. 564-587, 1952. 
[8] D. Crighton and F. Leppington, "Scattering of aerodynamic noise by a semi-infinite compliant plate," Journal of Fluid Mechanics, vol. 43, no. 4, pp. 721-736, 1970.

[9] M. Glauert and M. J. Lighthill, "The axisymmetric boundary layer on a long thin cylinder," Proceedings of the Royal Society of London. Series A. Mathematical and Physical Sciences, vol. 230, no. 1181, pp. 188-203, 1955.

[10] F. Quentin and J. Szodruch, "Aeronautics and air transport: beyond vision 2020 (towards 2050)," Report for ACARE, 2010.

[11] F. Mas, J. Menéndez, M. Oliva, and J. Ríos, "Collaborative engineering: an airbus case study," Procedia Engineering, vol. 63, no. 0, pp. 336-345, 2013.

[12] S.-J. Lee, P. Mulay, B. Diebolt-Brown, M. J. Lackovic, L. N. Mehler, J. Beckman, J. Waltz, J. B. Prado, Y. A. Mitchell, S. A. Higgins et al., "Acute illnesses associated with exposure to fipronilâsurveillance data from 11 states in the united states, 20012007," Clinical Toxicology, vol. 48, no. 7, pp. 737-744, 2010.

[13] M. J. Smith, Aircraft noise. Cambridge University Press, 2004, no. 3.

[14] M. Kaltenbach, C. Maschke, and R. Klinke, "Health consequences of aircraft noise," Deutsches Ärzteblatt International, vol. 105, no. 31-32, p. 548, 2008.

[15] L. Jarup, W. Babisch, D. Houthuijs, G. Pershagen, K. Katsouyanni, E. Cadum, M.-L. Dudley, P. Savigny, I. Seiffert, W. Swart et al., "Hypertension and exposure to noise near airports: the hyena study," Environmental health perspectives, vol. 116, no. 3, pp. 329-333, 2007.

[16] A. L. Hansell, M. Blangiardo, L. Fortunato, S. Floud, K. de Hoogh, D. Fecht, R. E. Ghosh, H. E. Laszlo, C. Pearson, L. Beale et al., "Aircraft noise and cardiovascular disease near heathrow airport in london: small area study," $B m j$, vol. 347 , p. f5432, 2013. 
[17] A. W. Correia, J. L. Peters, J. I. Levy, S. Melly, and F. Dominici, "Residential exposure to aircraft noise and hospital admissions for cardiovascular diseases: multiairport retrospective study," Bmj, vol. 347, p. f5561, 2013.

[18] C. Grimwood, C. Skinner, G. Raw, and M. Ling, "The uk national noise attitude survey 1999/2000-preliminary findings," in INTER-NOISE and NOISE-CON Congress and Conference Proceedings, vol. 2002, no. 4. Institute of Noise Control Engineering, 2002, pp. 1627-1632.

[19] C. A. A. Civil Aviation Authority (UK) Insight Note, "aviation policy for the environment," Tech. Rep., 2011.

[20] R. Ewert, J. Dierke, J. Siebert, A. Neifeld, C. Appel, M. Siefert, and O. Kornow, "Caa broadband noise prediction for aeroacoustic design," Journal of sound and vibration, vol. 330, no. 17, pp. 4139-4160, 2011.

[21] H. K. Ozcan and S. Nemlioglu, "In-cabin noise levels during commercial aircraft flights," Canadian Acoustics, vol. 34, no. 4, pp. 31-35, 2006.

[22] A. T. A. G. ATAG, "Aviation benefits beyond borders," 2014.

[23] F. A. F. Years, "Years 2016-2036. 2016," US Department of Transportation Federal Aviation Administration.

[24] R. Girvin, "Aircraft noise-abatement and mitigation strategies," Journal of Air Transport Management, vol. 15, no. 1, pp. 14-22, 2009.

[25] D. Casalino, F. Diozzi, R. Sannino, and A. Paonessa, "Aircraft noise reduction technologies: a bibliographic review," Aerospace Science and Technology, vol. 12, no. 1, pp. 1-17, 2008.

[26] W. Dobrzynski, "Almost 40 years of airframe noise research: what did we achieve?" Journal of aircraft, vol. 47, no. 2, pp. 353-367, 2010. 
[27] R. Blockley, R. Agarwal, F. Collier, A. Schaefer, and A. Seabridge, Green Aviation. John Wiley \& Sons, 2016.

[28] B. Al Tlua and R. Joana, "Optimization and testing of flat-plate trailing-edge serration geometry for reducing airfoil self-noise," Canadian Acoustics, vol. 48, no. 4, pp. $7-18,2020$.

[29] B. Al Tlua and J. Rocha, "Development and testing of an aeroacoustic wind tunnel test section," Canadian Acoustics, vol. 47, no. 3, pp. 64-65, 2019.

[30] S. M. Kuo and D. R. Morgan, "Active noise control: a tutorial review," Proceedings of the IEEE, vol. 87, no. 6, pp. 943-973, 1999.

[31] S. Wagner, R. Bareiss, and G. Guidati, Wind turbine noise. Springer Science \& Business Media, 2012.

[32] D. M. Chase, "Noise radiated from an edge in turbulent flow," AIAA journal, vol. 13, no. 8, pp. 1041-1047, 1975.

[33] R. K. Amiet, "Noise due to turbulent flow past a trailing edge," Journal of sound and vibration, vol. 47, no. 3, pp. 387-393, 1976.

[34] D. M. Chase, "Sound radiated by turbulent flow off a rigid half-plane as obtained from a wavevector spectrum of hydrodynamic pressure," The Journal of the Acoustical Society of America, vol. 52, no. 3B, pp. 1011-1023, 1972.

[35] M. S. Howe, "Aerodynamic noise of a serrated trailing edge," Journal of Fluids and Structures, vol. 5, no. 1, pp. 33-45, 1991.

[36] — - "Noise produced by a sawtooth trailing edge," The Journal of the Acoustical Society of America, vol. 90, no. 1, pp. 482-487, 1991. 
[37] M. Gruber, M. Azarpeyvand, and P. F. Joseph, "Airfoil trailing edge noise reduction by the introduction of sawtooth and slitted trailing edge geometries," integration, vol. 10, p. 6, 2010.

[38] B. Lyu, M. Azarpeyvand, and S. Sinayoko, "Prediction of noise from serrated trailing edges," Journal of Fluid Mechanics, vol. 793, pp. 556-588, 2016.

[39] G. Guidati, S. Wagner, R. Parchen, S. Oerlemans, R. Van den Berg, G. Schepers, K. Braun, and J. Kooi, "Design and testing of acoustically optimized airfoils for wind turbines (data)," in EWEC-CONFERENCE-, 1999, pp. 101-104.

[40] G. Guidati and S. Wagner, "Design of reduced noise airfoils for wind turbines," in Proc. of the ECCOMAS 2000 Conference (Barcelona, Spain), 2000.

[41] P. Fuglsang and H. A. Madsen, "Optimization method for wind turbine rotors," Journal of Wind Engineering and Industrial Aerodynamics, vol. 80, no. 1-2, pp. 191-206, 1999.

[42] A. L. Marsden, M. Wang, B. Mohammadi, and P. Moin, "Shape optimization for aerodynamic noise control," Center for Turbulence Research Annual Brief, pp. 24147, 2001.

[43] A. L. Marsden, M. Wang, J. E. Dennis, and P. Moin, "Optimal aeroacoustic shape design using the surrogate management framework," Optimization and Engineering, vol. 5, no. 2, pp. 235-262, 2004.

[44] A. L. Marsden, M. Wang, J. Dennis, and P. Moin, "Trailing-edge noise reduction using derivative-free optimization and large-eddy simulation," Journal of Fluid Mechanics, vol. 572, pp. 13-36, 2007.

[45] T. Lutz, W. Würz, A. Herrig, K. Braun, and S. Wagner, "Numerical optimization of silent airfoil sections," in 7th German Wind Energy Conference Proceedings, 2004. 
[46] T. Dassen, R. Parchen, J. Bruggeman, and F. Hagg, "Results of a wind tunnel study on the reduction of airfoil self-noise by the application of serrated blade trailing edges," in Proc. of the European Union Wind Energy Conference and Exhibition (Göteborg, Sweden), 1996.

[47] R. Parchen, W. Hoffmans, A. Gordner, and K. Braun, "Reduction of airfoil self-noise at low mach number with a serrated trailing edge," in International Congress on Sound and Vibration, 6 th, Technical Univ. of Denmark, Lyngby, Denmark, 1999, pp. 3433-3440.

[48] S. Oerlemans, M. Fisher, T. Maeder, and K. Kögler, "Reduction of wind turbine noise using optimized airfoils and trailing-edge serrations," AIAA journal, vol. 47, no. 6, pp. 1470-1481, 2009.

[49] U. Emborg, F. Samuelsson, J. Holmgren, and S. Leth, "Active and passive noise control in practice on the saab 2000 high speed turboprop," in 4th AIAA/CEAS aeroacoustics conference, 1998, p. 2231.

[50] C. N. Hansen, Understanding active noise cancellation. CRC Press, 1999.

[51] R. A. Kroeger, H. D. Grushka, and T. C. Helvey, "Low speed aerodynamics for ultra-quiet flight," TENNESSEE UNIV SPACE INST TULLAHOMA, Tech. Rep., 1972 .

[52] T. Bachmann and H. Wagner, "Silent owl wings," Encyclopedia of Nanotechnology, pp. 2411-2420, 2012.

[53] M. Herr, "Design criteria for low-noise trailing-edges," in 13th AIAA/CEAS Aeroacoustics Conference (28th AIAA Aeroacoustics Conference), 2007, p. 3470. 
[54] A. Powell, "On the aerodynamic noise of a rigid flat plate moving at zero incidence," The Journal of the Acoustical Society of America, vol. 31, no. 12, pp. 1649-1653, 1959.

[55] M. S. Howe, "A review of the theory of trailing edge noise," Journal of sound and vibration, vol. 61, no. 3, pp. 437-465, 1978.

[56] J. F. Williams and L. Hall, "Aerodynamic sound generation by turbulent flow in the vicinity of a scattering half plane," Journal of fluid mechanics, vol. 40, no. 4, pp. 657-670, 1970.

[57] M. Fink and D. BAILEY, "Model tests of airframe noise reduction concepts," in 6th Aeroacoustics Conference, 1980, p. 979.

[58] M. Herr and W. Dobrzynski, "Experimental investigations in low-noise trailing edge design." AIAA journal, vol. 43, no. 6, pp. 1167-1175, 2005.

[59] M. Gruber, "Airfoil noise reduction by edge treatments," Ph.D. dissertation, University of Southampton, 2012.

[60] M. S. Howe, "Aerodynamic noise of a serrated trailing edge," Journal of Fluids and Structures, vol. 5, no. 1, pp. 33-45, 1991.

[61] M. Herr, "A noise reduction study on flow-permeable trailing-edges," in Conference Proceedings of the Eighth ONERA-DLR Aerospace Symposium (ODAS), vol. 17. Citeseer, 2007, p. 19.

[62] T. P. Chong and A. Vathylakis, "On the aeroacoustic and flow structures developed on a flat plate with a serrated sawtooth trailing edge," Journal of Sound and Vibration, vol. 354, pp. 65-90, 2015. 
[63] F. Bertagnolio, H. A. Madsen, and C. Bak, "Trailing edge noise model validation and application to airfoil optimization," Journal of Solar Energy Engineering, vol. 132, no. 3, p. 031010, 2010.

[64] Z. Xiao, J. Liu, K. Luo, J. Huang, and S. Fu, "Investigation of flows around a rudimentary landing gear with advanced detached-eddy-simulation approaches," AIAA journal, vol. 51, no. 1, pp. 107-125, 2013.

[65] B. S. Lazos, "Mean flow features around the inline wheels of four-wheel landing gear," AIAA journal, vol. 40, no. 2, pp. 193-198, 2002.

[66] T. Kim, M. Jeon, S. Lee, and H. Shin, "Numerical simulation of flatback airfoil aerodynamic noise," Renewable energy, vol. 65, pp. 192-201, 2014.

[67] M. Murayama, Y. Yokokawa, K. Yamamoto, and T. Hirai, "Computational study of low-noise fairings around tire-axle region of a two-wheel main landing gear," Computers \&5 Fluids, vol. 85, pp. 114-124, 2013.

[68] M. Remillieux, E. Crede, H. Camargo, R. Burdisso, W. Devenport, M. Rasnick, P. Van Seeters, and A. Chou, "Calibration and demonstration of the new virginia tech anechoic wind tunnel," in 14th AIAA/CEAS Aeroacoustics Conference (29th AIAA Aeroacoustics Conference), 2008, p. 2911.

[69] D. Chase, "The character of the turbulent wall pressure spectrum at subconvective wavenumbers and a suggested comprehensive model," Journal of Sound and Vibration, vol. 112, no. 1, pp. 125-147, 1987.

[70] R. Wahidi, W. Chakroun, and S. Al-Fahed, "The behavior of the skin-friction coefficient of a turbulent boundary layer flow over a flat plate with differently configured transverse square grooves," Experimental Thermal and Fluid Science, vol. 30, no. 2, pp. 141-152, 2005. 
[71] D. A. Bies, "A review of flight and wind tunnel measurements of boundary layer pressure fluctuations and induced structural response," 1966.

[72] M.-S. Kim, J.-H. Lee, J.-D. Kee, and J.-H. Chang, "Hyundai full scale aero-acoustic wind tunnel," SAE Technical Paper, Tech. Rep., 2001.

[73] E. Sarradj, C. Fritzsche, T. Geyer, and J. Giesler, "Acoustic and aerodynamic design and characterization of a small-scale aeroacoustic wind tunnel," Applied Acoustics, vol. 70, no. 8, pp. 1073-1080, 2009.

[74] T. MAEDA and Y. KONDO, "Rtri's large-scale low-noise wind tunnel and wind tunnel tests," Quarterly Report of RTRI, vol. 42, no. 2, pp. 65-70, 2001.

[75] T. Chong, P. Joseph, and P. Davies, "Design and performance of an open jet wind tunnel for aero-acoustic measurement," Applied acoustics, vol. 70, no. 4, pp. 605$614,2009$.

[76] M. Gruber, P. Joseph, and M. Azarpeyvand, "An experimental investigation of novel trailing edge geometries on airfoil trailing edge noise reduction," in 19th AIAA/CEAS aeroacoustics conference, 2013, p. 2011.

[77] L. Jones and R. Sandberg, "Numerical investigation of airfoil self-noise reduction by addition of trailing-edge serrations," in 16th AIAA/CEAS Aeroacoustics Conference, 2010, p. 3703 .

[78] A. T. A. G. ATAG, "Aviation benefits beyond borders," 2014.

[79] D. A. Bies and C. H. Hansen, Engineering noise control: theory and practice. CRC press, 2017.

[80] M. C. Madahana, N. F. Moroe, and O. T. Nyandoro, "Engineering noise control for mines: Lessons from the world," South African Journal of Communication Disorders, vol. 67, no. 2, pp. 1-5, 2020 . 
[81] K. Schwarzschild, "Sitzungsber. deutsch. akad," Wissenschaft Berlin Kl. Math. Phys. Tech, vol. 424, 1916.

[82] J. Narlikar and T. Padmanabhan, "The schwarzschild solution: some conceptual difficulties," Foundations of physics, vol. 18, no. 6, pp. 659-668, 1988.

[83] E. Sarradj, C. Fritzsche, T. Geyer, and J. Giesler, "Acoustic and aerodynamic design and characterization of a small-scale aeroacoustic wind tunnel," Applied Acoustics, vol. 70, no. 8, pp. 1073-1080, 2009.

[84] U. Diwekar, Introduction to applied optimization. Springer Science \& Business Media, 2008, vol. 22.

[85] L. Yong, "Interior point algorithms and applications," in Future Intelligent Information Systems. Springer, 2011, pp. 165-172.

[86] N. Karmarkar, "A new polynomial-time algorithm for linear programming," in Proceedings of the sixteenth annual ACM symposium on Theory of computing. ACM, 1984, pp. 302-311.

[87] C. Roos, T. Terlaky, and J.-P. Vial, Interior point methods for linear optimization. Springer Science \& Business Media, 2005.

[88] I. Farhat and M. El-Hawary, "Interior point methods application in optimum operational scheduling of electric power systems," IET generation, transmission 8 distribution, vol. 3, no. 11, pp. 1020-1029, 2009.

[89] A. D. Nguyen, A. Hachemi, and D. Weichert, "Application of the interior-point method to shakedown analysis of pavements," International Journal for Numerical Methods in Engineering, vol. 75, no. 4, pp. 414-439, 2008. 
[90] S. Granville, J. Mello, and A. Melo, "Application of interior point methods to power flow unsolvability," IEEE Transactions on Power Systems, vol. 11, no. 2, pp. 1096$1103,1996$.

[91] S. Narayanan, P. Chaitanya, S. Haeri, P. Joseph, J. Kim, and C. Polacsek, "Airfoil noise reductions through leading edge serrations," Physics of Fluids, vol. 27, no. 2, p. $025109,2015$.

[92] C. P. Ortega, "Chapter 2: Effects of noise pollution on birds: A brief review of our knowledge," Ornithological monographs, vol. 74, no. 1, pp. 6-22, 2012.

[93] A. L. Bronzaft, "Noise pollution: A threat to our mental and physical well-being," The Journal of the Acoustical Society of America, vol. 115, no. 5, pp. 2567-2567, 2004.

[94] G. Naterer, O. Jianu, and M. Rosen, "Noise polution prevention in wind turbines: Status and recent advances," 2011.

[95] R. Graham, "The silent flight of owls," The Aeronautical Journal, vol. 38, no. 286, pp. 837-843, 1934.

[96] G. Lilley, "A study of the silent flight of the owl," in 4th AIAA/CEAS aeroacoustics conference, 1998, p. 2340.

[97] T. P. Chong, A. Vathylakis, P. F. Joseph, and M. Gruber, "Self-noise produced by an airfoil with nonflat plate trailing-edge serrations," AIAA journal, vol. 51, no. 11, pp. 2665-2677, 2013.

[98] E. Chen, Y. Ma, A. Yang, and G. Zhao, "Experimental investigation on noise emissions of an airfoil with non-flat plate trailing edge serrations," Journal of Mechanical Science and Technology, vol. 33, no. 7, pp. 3069-3074, 2019. 
[99] E. Manoha, B. Troff, and P. Sagaut, "Trailing-edge noise prediction using large-eddy simulation and acoustic analogy," AIAA journal, vol. 38, no. 4, pp. 575-583, 2000.

[100] A. Bodling, B. R. Agrawal, A. Sharma, I. Clark, W. N. Alexander, and W. J. Devenport, "Numerical investigations of bio-inspired blade designs to reduce broadband noise in aircraft engines and wind turbines," in 55th AIAA Aerospace Sciences Meeting, 2017, p. 0458.

[101] H. Tang, Y. Lei, and Y. Fu, "Noise reduction mechanisms of an airfoil with trailing edge serrations at low mach number," Applied Sciences, vol. 9, no. 18, p. 3784, 2019.

[102] A. Zilstra and D. Johnson, "Les and fw-h prediction of aeroacoustic noise for a sd 7037 airfoil for wind turbine applications," in 25th AIAA/CEAS Aeroacoustics Conference, 2019, p. 2537.

[103] M. P. Rumpfkeil, "Using steady flow analysis for noise predictions," Computers \& Fluids, vol. 154, pp. 347-357, 2017.

[104] S. Hosder, J. A. Schetz, W. H. Mason, B. Grossman, and R. T. Haftka, "Computational-fluid-dynamics-based clean-wing aerodynamic noise model for design," Journal of aircraft, vol. 47, no. 3, pp. 754-762, 2010.

[105] C. Albarracin, C. Doolan, R. Jones, C. Hansen, L. Brooks, and M. Teubner, "A rans-based statistical noise model for trailing edge noise," in 18th AIAA/CEAS Aeroacoustics Conference (33rd AIAA Aeroacoustics Conference), 2012, p. 2181.

[106] S. Remmler, J. Christophe, J. Anthoine, and S. Moreau, "Computation of wall pressure spectra from steady flow data for noise prediction," AIAA journal, vol. 48, no. 9, pp. 1997-2007, 2010.

[107] P. Qumr and P. Sagaut, "Zonal multi-domain rans/les simulations of turbulent ows," Int J Numer Methods Fluids, vol. 40, no. 7, p. 903925, 2002. 
[108] M. Terracol, "A zonal rans/les approach for noise sources prediction," Flow, turbulence and combustion, vol. 77, no. 1-4, pp. 161-184, 2006.

[109] J. Fröhlich and D. Von Terzi, "Hybrid les/rans methods for the simulation of turbulent flows," Progress in Aerospace Sciences, vol. 44, no. 5, pp. 349-377, 2008.

[110] F. Mathey, "Aerodynamic noise simulation of the flow past an airfoil trailing-edge using a hybrid zonal rans-les," Computers \& Fluids, vol. 37, no. 7, pp. 836-843, 2008.

[111] G. Lane, P. Croaker, and Y. Ding, "Embedded large eddy simulation method for predicting flow-induced noise," in Proceedings of ACOUSTICS, vol. 10, no. 13, 2019.

[112] Z. Zuo, Q. Huang, and S. Liu, "An analysis on the flow field structures and the aerodynamic noise of airfoils with serrated trailing edges based on embedded large eddy flow simulations," Journal of Applied Fluid Mechanics, vol. 12, no. 2, pp. 327-339, 2019.

[113] A. Leonard, "Energy cascade in large-eddy simulations of turbulent fluid flows," in Advances in geophysics. Elsevier, 1975, vol. 18, pp. 237-248.

[114] J. Hinze and M. Uberoi, "Turbulence," Journal of Applied Mechanics, vol. 27, no. 3, p. $601,1960$.

[115] F. Nicoud and F. Ducros, "Subgrid-scale stress modelling based on the square of the velocity gradient tensor," Flow, turbulence and Combustion, vol. 62, no. 3, pp. 183-200, 1999.

[116] F. Mathey, D. Cokljat, J.-P. Bertoglio, and E. Sergent, "Specification of les inlet boundary condition using vortex method," Progress in Computational Fluid Dynamics, vol. 6, pp. 58-67, 2006. 
[117] J. F. Williams and D. L. Hawkings, "Sound generation by turbulence and surfaces in arbitrary motion," Philosophical Transactions for the Royal Society of London. Series A, Mathematical and Physical Sciences, pp. 321-342, 1969.

[118] K. B. F. Farassat and K. Brentner, "An analytical comparison of the acoustic analogy and kirchhoff formulation for moving surface," AIAA J, vol. 36, pp. 1379-1386, 1998.

[119] M. Berger and M. Aftosmis, "Progress towards a cartesian cut-cell method for viscous compressible flow," in 50th AIAA Aerospace Sciences Meeting Including the New Horizons Forum and Aerospace Exposition, 2012, p. 1301.

[120] R. E. Ghmati, B. A. Jawad, and E. Koutsavdis, "An investigation of cutcell meshing strategies for accurate aerodynamic performance prediction," SAE International Journal of Passenger Cars-Mechanical Systems, vol. 5, no. 2012-01-0499, pp. 369$380,2012$.

[121] F. R. Menter, "Best practice: scale-resolving simulations in ansys cfd," ANSYS Germany GmbH, vol. 1, 2012.

[122] F. ANSYS, "Ansys fluent theory guide 19.1," ANSYS, Canonsburg, PA, 2019.

[123] F. R. Menter, "Two-equation eddy-viscosity turbulence models for engineering applications," AIAA journal, vol. 32, no. 8, pp. 1598-1605, 1994.

[124] F. H. Clauser, "The turbulent boundary layer," in Advances in applied mechanics. Elsevier, 1956, vol. 4, pp. 1-51.

[125] V. Falkneb and S. W. Skan, "Lxxxv. solutions of the boundary-layer equations," The London, Edinburgh, and Dublin Philosophical Magazine and Journal of Science, vol. 12 , no. 80, pp. 865-896, 1931. 
[126] H. Ludwieg, Instrument for measuring the wall shearing stress of turbulent boundary layers. National Advisory Committee for Aeronautics, 1950.

[127] H. Lee and S.-H. Kang, "Flow characteristics of transitional boundary layers on an airfoil in wakes," J. Fluids Eng., vol. 122, no. 3, pp. 522-532, 2000.

[128] O. Marsden, C. Bogey, and C. Bailly, "Direct noise computation around a 3-d naca 0012 airfoil," in 12th AIAA/CEAS Aeroacoustics Conference (27th AIAA Aeroacoustics Conference), 2006, p. 2503.

[129] M. S. Chong, A. E. Perry, and B. J. Cantwell, "A general classification of threedimensional flow fields," Physics of Fluids A: Fluid Dynamics, vol. 2, no. 5, pp. $765-777,1990$.

[130] R. Green and J. Gerrard, "Vorticity measurements in the near wake of a circular cylinder at low reynolds numbers," Journal of Fluid Mechanics, vol. 246, pp. 675$691,1993$.

[131] J. Gerrard, "The mechanics of the formation region of vortices behind bluff bodies," Journal of fluid mechanics, vol. 25, no. 2, pp. 401-413, 1966.

[132] R. D. Blevins, "Flow-induced vibrations. reprint of the 1990," 2001.

[133] J. Hu, Z. Wang, W. Zhao, S. Sun, C. Sun, and C. Guo, "Numerical simulation on vortex shedding from a hydrofoil in steady flow," Journal of Marine Science and Engineering, vol. 8, no. 3, p. 195, 2020.

[134] W. H. Press, H. William, S. A. Teukolsky, W. T. Vetterling, A. Saul, and B. P. Flannery, Numerical recipes 3rd edition: The art of scientific computing. Cambridge university press, 2007.

[135] V. Strouhal, Über eine besondere Art der Tonerregung. Stahel, 1878. 
[136] M. J. Kingan and J. R. Pearse, "Laminar boundary layer instability noise produced by an aerofoil," Journal of Sound and Vibration, vol. 322, no. 4-5, pp. 808-828, 2009.

[137] E. C. Nash, M. V. Lowson, and A. McAlpine, "Boundary-layer instability noise on aerofoils," Journal of Fluid Mechanics, vol. 382, pp. 27-61, 1999.

[138] C. K. Tam, "Discrete tones of isolated airfoils," The Journal of the Acoustical Society of America, vol. 55, no. 6, pp. 1173-1177, 1974.

[139] A. Finez, M. Jacob, E. Jondeau, and M. Roger, "Broadband noise reduction with trailing edge brushes," in 16th AIAA/CEAS aeroacoustics conference, 2010, p. 3980.

[140] T. Geyer, E. Sarradj, and C. Fritzsche, "Measurement of the noise generation at the trailing edge of porous airfoils," Experiments in fluids, vol. 48, no. 2, pp. 291-308, 2010 .

[141] D. J. Moreau and C. J. Doolan, "Noise-reduction mechanism of a flat-plate serrated trailing edge," AIAA journal, vol. 51, no. 10, pp. 2513-2522, 2013.

[142] M. Lowson, S. Fiddes, and E. Nash, "Laminar boundary layer aero-acoustic instabilities," in 32nd Aerospace Sciences Meeting and Exhibit, 1994, p. 358.

[143] G. Desquesnes, M. Terracol, and P. Sagaut, "Numerical investigation of the tone noise mechanism over laminar airfoils," Journal of Fluid Mechanics, vol. 591, p. $155,2007$.

[144] R. W. Paterson, P. G. Vogt, M. R. Fink, and C. L. Munch, "Vortex noise of isolated airfoils," Journal of Aircraft, vol. 10, no. 5, pp. 296-302, 1973.

[145] R. Dieck, W. Steele, and G. Osolsobe, "Test uncertainty. asme ptc 19.1-2005," American Society of Mechanical Engineers, New York, NY, 2005. 
[146] H. W. Coleman and W. G. Steele, "Engineering application of experimental uncertainty analysis," AIAA journal, vol. 33, no. 10, pp. 1888-1896, 1995. 


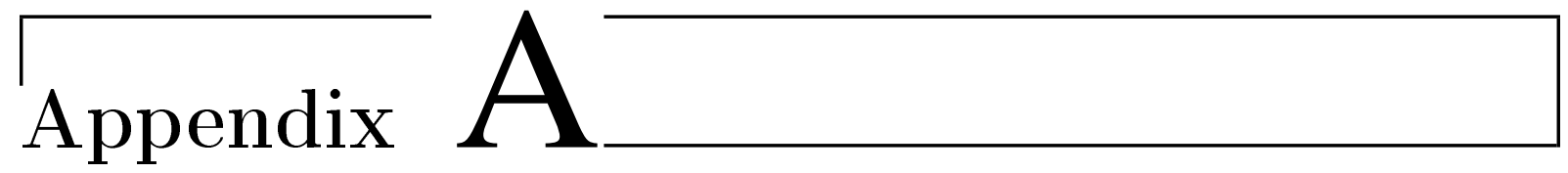

\section{Measurement Uncertainty}

The uncertainty of any measurement comes from two sources of errors [145]; first: systematic, bias, errors related to the hardware used to acquire data, such as data acquisition system, microphone junction, extension wires, and connectors. This type of error is usually constant throughout the experiment. The second source is called random error which is proportional inversely to the number of experiment repeats at the same condition. This section explains the method used to calculate random uncertainty and presents types of bias errors considered to get bias uncertainty.

There is a number of methods used to calculate total uncertainty, and they are well explained in reference [146], by the American Society of Mechanical Engineers (ASME). This method is defined by:

$$
T U_{A S M E}= \pm t_{95}\left[\left(B_{T} / 2\right)^{2}+\left(R_{T}\right)^{2}\right]^{0.5}
$$

where $T U_{A S M E}$ is the total uncertainty of the measurement, $B_{T}$ is the total bias uncertainty, $R_{T}$ is the total random uncertainty, and $t_{95}$ is the student's $t$ value at $95 \%$ confidence. For a number of repeats equals 5 ( with a degree of freedom $N-1=4$ ), $t_{95}=2.776$. The total bias uncertainty $\left(B_{T}\right)$ is calculated (see the Table A.1) by using root sum squared as shown in the Equation A.2. 


$$
B_{T}=\sqrt{B_{1}^{2}+B_{2}^{2}+B_{3}^{2}+B_{4}^{2}}
$$

The total random uncertainty $\left(R_{T}\right)$ is found by using standard deviation method defined by Equation A.3

$$
R_{T}=\sqrt{\frac{\sum_{i=1}^{N}\left(P_{i}-\bar{P}\right)^{2}}{N-1}}
$$

where $N$ is the number of repeats, $P_{i}$ is microphone reading, noise level $(\mathrm{dB})$, and $\bar{P}$ is the mean of microphone readings, noise level $(\mathrm{dB})$.

Table A.1: Sources of systematic uncertainties with total bias uncertainty

\begin{tabular}{ccc}
\hline Hardware name & Type of source error & Bias uncertainty $\pm \%$ \\
\hline \hline Data acquisition system & $\left(B_{1}\right)$ & 0.10 \\
\hline \multirow{2}{*}{ Microphone } & Mic. accuracy $\left(B_{2}\right)$ & 0.83 \\
\cline { 2 - 3 } & Mic. extension wire $\left(B_{3}\right)$ & 0.33 \\
\hline Mic. connector & connector $\left(B_{4}\right)$ & 0.07 \\
\hline \multicolumn{2}{c}{ Total bias uncertainty $\left(B_{T}\right)$} & 0.81 \\
\hline
\end{tabular}

An estimate for the uncertainty in the noise level is made based on repeated measurements. Several measurements of the noise were made over a range of test section velocities. The uncertainties presented are averaged over the entire frequency range $(0.1$ $\mathrm{kHz}-10 \mathrm{kHz}$ ). The Table A.2 presents the calculated uncertainties for noise levels as a function of flow speed. 
Table A.2: Uncertainties in noise level.

\begin{tabular}{cc}
\hline Test section velocity, $U_{\infty}(\mathrm{m} / \mathrm{s})$ & Uncertainty $( \pm \mathrm{dB})$ \\
\hline 7 & 1.12 \\
9 & 1.18 \\
12.7 & 1.25 \\
14 & 1.30 \\
16 & 1.36 \\
18 & 1.40 \\
22 & 1.47 \\
24 & 1.51 \\
26.7 & 1.58 \\
\hline
\end{tabular}

